\title{
6 Selbstreparaturen in der Kurznachrichtenkommunikation
}

\subsection{Selbstinitiierte Selbstreparaturen in der Kurznachrichtenkommunikation}

Das deutsche Analysekorpus weist insgesamt $223^{96}$ selbstinitiierte Selbstreparaturen auf, während sich deren Anzahl in den russischen Daten auf 59 Fälle beläuft. Am häufigsten sind selbstinitiierte Selbstreparaturen im sogenannten next opportunity space (vgl. Kap. 6.1.1) vertreten, also diejenigen, die in der nächsten Nachricht des Produzenten der Problemquelle durchgeführt werden: 153 Vorkommnisse in den deutschen und 42 in den russischen Kurznachrichtendialogen machen jeweils ca. $69 \%$ bzw. $71 \%$ der Gesamtanzahl selbstinitiierter Selbstreparaturen in dem entsprechenden Subkorpus aus. Diese Gruppe subsumiert auch diejenigen selbstinitiierten Selbstreparaturen (15 im deutschen und vier im russischen Subkorpus), deren Notwendigkeit vom Produzenten der Problemquelle erst an der Reaktion des Rezipienten erkannt wurde, also third position repairs im Sinne von Schegloff (1987b, 1992), auf die im weiteren Verlauf der Arbeit als Missverständnisse im next opportunity space referiert wird und die als besondere Gruppe von selbstinitiierten Selbstreparaturen im next opportunity space gesondert im Kapitel 6.1.4 behandelt werden.

Nach selbstinitiierten Selbstreparaturen im next opportunity space bilden Reparaturen im same turn die zweithäufigste Gruppe. 60 Reparaturen in den deutschen und 14 Reparaturen in den russischen Nachrichten (ca. 27\% bzw. 24\%) erfolgen in der gleichen Nachricht, in der sich ebenfalls die entsprechende Problemquelle befindet.

Am seltensten treten in den analysierten Daten after next opportunity space repairs auf, also Reparaturen, bei denen zwischen der Nachricht mit dem Reparandum und der mit der Reparaturdurchführung mindestens eine weitere Nachricht des Produzenten der Problemquelle vorhanden ist. Solche Reparaturen liegen lediglich in neun Fällen im deutschen und in drei Fällen im russischen Subkorpus vor, was jeweils 4\% bzw. 5\% der gesamten Anzahl selbstinitiierter Selbstreparaturen entspricht.

96 Bei einer selbstinitiierten Selbstreparatur aus dem deutschen Subkorpus ist die Problemquelle in dem zur Verfügung stehenden Dialogverlauf nicht abgebildet, sodass die Position der vorliegenden Reparatur nicht zu ermitteln ist. Dieser Fall wird daher aus der Berechnung der Anzahl von Reparaturen in Abhängigkeit von ihrer Position ausgeschlossen. 
Auf den formalen Aufbau sowie auf die interaktionalen Funktionen von selbstinitiierten Selbstreparaturen in diesen drei Positionen wird in Kapiteln 6.1.1-6.1.3 eingegangen. Dabei orientiert sich die folgende Darstellung an den Häufigkeiten der Reparaturen in der jeweiligen Position im Untersuchungskorpus und nicht an deren natürlicher sequenzieller Reihenfolge, sodass zuerst nicht Reparaturen im same turn, sondern diejenigen im next opportunity space diskutiert werden. Diese Vorgehensweise ist v.a. dadurch motiviert, dass sich die mit Abstand größte Gruppe von selbstinitiierten Selbstreparaturen im next opportunity space am besten für die Kategorienbildung anbietet, welche auf die Analyse von Reparaturen im same turn sowie im after next opportunity space übertragen werden können. Den Missverständnissen im next und after next opportunity space, die sich im Gegensatz $\mathrm{zu}$ sonstigen selbstinitiierten Selbstreparaturen durch die Relevanz der Rezipientenreaktion für das Auftreten einer Reparatur auszeichnen, was sich letztendlich in den formalen sowie funktionalen Eigenschaften solcher Reparaturen niederschlägt, widmet sich Kapitel 6.1.4.

\subsubsection{Selbstinitiierte Selbstreparaturen im next opportunity space}

\subsubsection{Reparanda}

Die im Analysekorpus auftretenden Problemquellen werden in Anlehnung an Pfeiffer (2015: 54) in Abhängigkeit davon, welcher sprachlichen Ebene das jeweilige Reparandum aus der Rezipientenperspektive zugeordnet werden kann, in (ortho)graphische, lexiko-semantische, morphosyntaktische und pragmatische Problemquellen unterteilt.

\section{(i) (Ortho)graphische Reparanda}

Auf der (ortho)graphischen sprachlichen Ebene sind in den untersuchten Daten die Reparaturen angesiedelt, bei denen das auftretende Problem die (Recht)schreibung einzelner Lexeme oder Emoticons sowie die Zeichensetzung betrifft. Dabei ist aber hervorzuheben, dass es bei den korrigierten sprachlichen Ausdrücken nicht nur um diejenigen geht, welche aus der Sicht der normativen Grammatik im weiteren Sinne als fehlerhaft gelten. In einzelnen Fällen richten sich Schreiber nicht nach der standardsprachlichen Norm, sondern nach einer gruppenspezifischen oder sogar lokal in einer Interaktion ad hoc gebildeten Norm, die gegen die standardschriftsprachlichen Regeln verstoßen kann (vgl. (88)). Daher wurde beschlossen, die in diesem Abschnitt thematisierten Reparanda nicht als orthographisch, sondern als (ortho)graphisch zu bezeichnen, 
um zu zeigen, dass diese Kategorie nicht nur orthographische Fehler im klassischen Sinne, sondern jegliche von Schreibern als problematisch angesehene graphische Repräsentationen einzelner Wortformen und Emoticons miteinschließt. Die Angemessenheit einer solchen ausgeweiteten Perspektive lässt sich anhand der Reparatur in (88) erkennen, in der das aus normativer Sicht korrekte Lexem „viewing“ (\#16) als Reparandum fungiert und durch die sich im vorherigen Verlauf der Interaktion etablierte ad hoc gebildete individuelle Variante „Iewing“ ersetzt wird:

(88) Auszug aus dem WhatsApp-Dialog \#3579:

Schreiberin $A$

Schreiberin $B$

\begin{tabular}{|c|}
\hline Guckst du jetzt eigentlich irgendwo Fußball heute? =D \\
\hline Nachricht \#1 - 12.07.2014 - 21:00:37 \\
\hline
\end{tabular}

\begin{tabular}{|c|}
\hline Ja zuhause mach ich public iuing =D und du? \\
\hline Nachricht \#2-12.07.2014 - 21:01:56 \\
\hline
\end{tabular}

\begin{tabular}{|c|}
\hline$=$ 'D das ist wirklich ein schönes Wort $=\mathrm{D}$ \\
\hline Nachricht \#3 $-12.07 .2014-21: 02: 12$ \\
\hline
\end{tabular}

\begin{tabular}{|c|}
\hline Ich auch zu hause $=\mathrm{D}$ \\
\hline Nachricht $\# 4-12.07 .2014-21: 02: 30$ \\
\hline
\end{tabular}

\begin{tabular}{|c|}
\hline Ja ich finde es sollte nie in Vergessenheit geraten $=$ ) \\
\hline Nachricht $\# 5-12.07 .2014-21: 02: 50$ \\
\hline
\end{tabular}

Ach ja beim spiel Platz drei iszs ja nicht si wichtig

Nachricht \#6 - 12.07.2014 - 21:03:10

\begin{tabular}{|c|}
\hline Das ist genau wie die pool position =’D \\
\hline Nachricht \#7 - 12.07.2014 - 21:03:17 \\
\hline
\end{tabular}

Ja das stimmt da dachte ich mir auch wirds schön gemütlich gemacht =)

Nachricht \#8 - 12.07.2014 - 21:03:25

Aber auf morgen freu ich mich auch schn $=\mathrm{D}$

Nachricht \#9 - 12.07.2014 - 21:03:34 


\begin{tabular}{|c|}
\hline Vllt sind bekannte da u dann ists wirklich public iuing =D \\
\hline Nachricht $\# 10-12.07 .2014-21: 03: 44$ \\
\hline
\end{tabular}

\begin{tabular}{|c|}
\hline Ja morgen wird super $=$ ) \\
\hline Nachricht \#11 - 12.07.2014 - 21:04:05 \\
\hline
\end{tabular}

Bei mir ist nur die family anwesend. Ich hab also quasi private ieving =D

Nachricht \#12 - 12.07.2014 - 21:05:15

\begin{tabular}{|c|}
\hline U ich public =`D \\
\hline Nachricht \#13 $-12.07 .2014-21: 14: 04$ \\
\hline
\end{tabular}

\begin{tabular}{|c|}
\hline Doch nicht.Die sind weg \\
\hline Nachricht \#14 - 12.07.2014 - 21:41:03 \\
\hline
\end{tabular}

\begin{tabular}{|c|}
\hline Oh hupswo sind sie denn \\
\hline Nachricht \#15 - 12.07.2014 - 21:41:10 \\
\hline
\end{tabular}

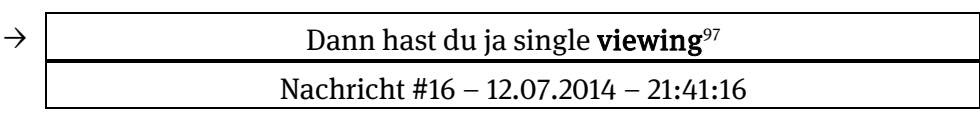

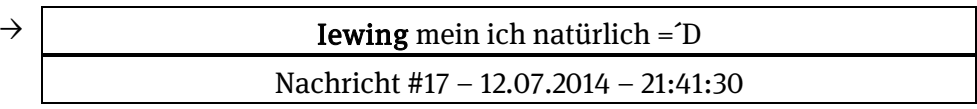

Nach hause gefahren also die bekannten. Meine Eltern sind da

Nachricht \#18 - 12.07.2014 - 21:41:40

\begin{tabular}{|c|}
\hline Ach soo =D ich dachte die wären jetzt alle weg =D \\
\hline Nachricht \#19 $-12.07 .2014-21: 42: 06$ \\
\hline
\end{tabular}

97 Für eine bessere Orientierung werden das Reparandum und das Reparans in den analysierten Kurznachrichtendialogen fett markiert. Die Pfeile markieren die jeweils im Fokus der Analyse stehende Reparatursequenz. Außerdem soll an dieser Stelle darauf hingewiesen werden, dass bei dem jeweiligen Beispiel - falls bekannt - angegeben wird, ob dieses aus einem SMS-, WhatsApp-, iMessage- oder Viber-Dialog stammt. Bei Fällen, in denen die Metadaten keine eindeutige Zuordnung des Dialogs einer dieser Kommunikations(platt)formen erlauben, erscheint lediglich die Angabe Dialog. 
In der initialen Nachricht des in (88) vorliegenden Auszugs erkundigt sich Schreiberin $A$, ob - und falls ja wo - sich die mit ihr befreundete Schreiberin $B$ das gerade ausgestrahlte Fußballspiel zwischen Brasilien und den Niederlanden um den dritten Platz anschaut, welches im Rahmen der Weltmeisterschaft 2014 stattfindet. Es stellt sich anschließend heraus, dass die beiden das Spiel von zu Hause aus verfolgen (vgl. \#2 und \#4), wobei Schreiberin $B$ ihre Art des Fußballschauens zu Hause als „public iuing“ (\#2) bezeichnet. Die von $B$ in \#2 für das Wort Viewing verwendete Form „iuing“, die aus normorthographischer Sicht nicht korrekt ist, wird von $A$ in \#3 positiv bewertet („='D das ist wirklich ein schönes Wort $=\mathrm{D}$ “). Daraufhin bestätigt $B A$ 's positive Bewertung (,Ja ich finde es sollte nie in Vergessenheit geraten $=$ )“ in \#5) und fördert die lokale Etablierung von „iuing“ dadurch, dass sie dieses im weiteren Verlauf des Dialogs (vgl. \#10) benutzt. ${ }^{98}$ Schreiberin $A$ versucht sich ebenso nach dieser lokal ad hoc gebildeten Konvention zu richten, was ihr allerdings nur zum Teil gelingt, da sie nicht ,iuing“, sondern „,ieving“ (\#12) realisiert. $A$ 's Orientierung an der lokal in der Interaktion etablierten Form iuing bzw. ieving kommt besonders deutlich durch die selbstinitiierte Selbstreparatur in \#17 zum Ausdruck, in der sie „,viewing“ aus \#16 als Reparandum behandelt und durch „Iewing“ ersetzt.

Des Weiteren werden (ortho)graphischen Reparanda solche Belege zugeordnet, in denen Schreiber unabsichtlich die falsche Formatierungseinstellung der Schrift eingesetzt oder die Eingabesprache nicht umgestellt haben. Im ersten Fall handelt es sich i.d.R. um die nicht-intendierte Verwendung der Majuskelschreibung (vgl. (57) sowie (121)). Im zweiten Fall betrifft das Problem diejenigen Schreiber, die die Desktop-Versionen der Messenger-Dienste nutzen sowie beim Schreiben zwischen mindestens zwei Sprachen mit unterschiedlichen Schriftsystemen und dementsprechend verschiedener Belegung der Tastatur wechseln. Wenn aber dabei aus Versehen die Sprache nicht umgeschaltet und dies vom Schreiber beim Eintippen einer Nachricht nicht bemerkt wird, entsteht eine sinnlose Kombination aus einzelnen Buchstaben oder sonstigen Zeichen, wie in (89):

98 Ein von Bücker (2004: 84-86) beschriebener Gesprächsausschnitt aus einer Fernsehdiskussionsrunde weist ein ähnliches Phänomen auf: Im Laufe der Diskussion wird eine neue, ad hoc gebildete und in dem Kontext rhetorisch passendere Variante des Phraseologismus eine Lanze brechen ausgehandelt, indem die Diminutivierung des Nomens Lanze zu Länzchen (ein Länzchen brechen) vorgenommen wird. Dieses etabliert sich schnell innerhalb der Sprechergruppe und wird im weiteren Verlauf des Gesprächs immer wieder eingesetzt. 
(89) Auszug aus dem WhatsApp-Dialog \#3348:

Schreiberin $A$

Schreiberin $B$

\begin{tabular}{|c|}
\hline Лови (Файл перевод.docx) \\
\hline Hier ist die Datei (Übersetzung.docx) \\
\hline Nachricht \#1 - 29.04.2014-14:05:05 \\
\hline
\end{tabular}

\begin{tabular}{|c|}
\hline thank u so muuuuch \\
\hline Ich danke dir soooo sehr \\
\hline Nachricht \#2 $-29.04 .2014-14: 06: 05$ \\
\hline
\end{tabular}

$\rightarrow$

\begin{tabular}{|c|}
\hline f xt nfr vfkj\& \\
\hline $\begin{array}{c}\text { (sinnlose Kombination aus den lateinischen Buchstaben und dem } \\
\text { \&-Zeichen) }\end{array}$ \\
\hline Nachricht \#3 - 29.04.2014-14:07:05 \\
\hline
\end{tabular}

$\rightarrow$

\begin{tabular}{|c|}
\hline там мало одна стр \\
\hline Die Datei enthält zu wenig Seiten, nur eine einzige. \\
\hline Nachricht \#4-29.04.2014-14:09:05 \\
\hline
\end{tabular}

\begin{tabular}{|c|}
\hline Да \\
\hline Ja \\
\hline Nachricht \#5 $-29.04 .2014-14: 11: 05$ \\
\hline
\end{tabular}

In \#1 leitet Schreiberin $A$ ihrer Kommilitonin eine Datei weiter, deren Inhalte für eine Lehrveranstaltung aufbereitet werden sollen. Schreiberin $B$ bedankt sich dafür auf Englisch (vgl. \#2). Dabei lässt sich die Sprachwahl vermutlich damit begründen, dass die beiden Studentinnen Romanistik und Anglistik studieren und es sich bei der in \#1 verschickten Datei anscheinend um eine Aufgabe für die nächste Unterrichtstunde in Englisch handelt. Beim Eintippen einer weiteren Nachricht - diesmal auf Russisch - merkt Schreiberin $B$ jedoch nicht, dass auf ihrem PC Englisch immer noch als Eingabesprache eingestellt ist. Die somit verfasste Nachricht \#3 (,f xt nfr vfkj\&“) ergibt daher keinen Sinn, sondern stellt lediglich eine Aneinanderreihung von lateinischen Buchstaben sowie dem und-Zeichen dar. Wenn man sich die Tastenbelegung einer russischen Tastatur (Abb. 2) anschaut, lässt sich der Nachrichteninhalt а че так мало? (,und wieso so wenig?`) dennoch problemlos rekonstruieren, indem jedem lateinischen Buchstaben ein kyrillischer zugeordnet wird und dem \&-Zeichen ein Fragezei- 
chen, das durch die Kombination der Alt-Taste und der Taste für 7 , die gleichzeitig gedrückt werden müssen, erzeugt wird.

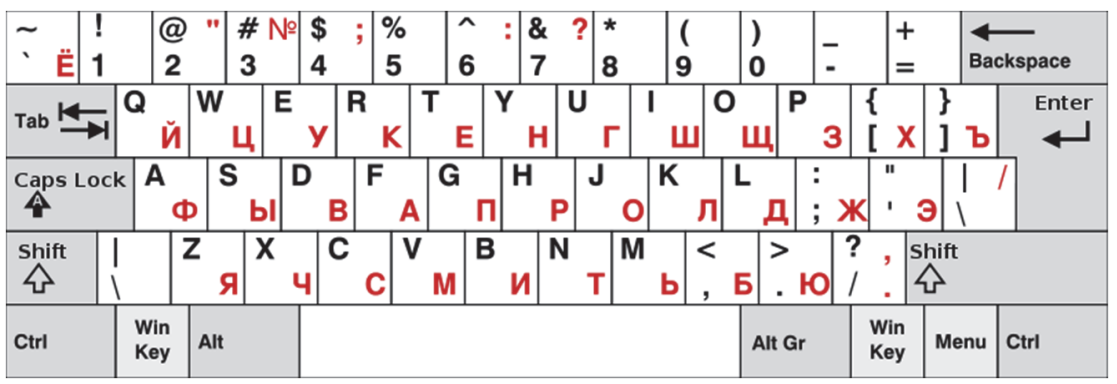

Abb. 2: PC-Tastatur mit der Tastenbelegung in kyrillischer und lateinischer Schrift (Quelle: russische Tastatur: Denelson83, amerikanische Tastatur: Stuart Brady, Bildbearbeitung durch: Quanthon (talk), https://commons.wikimedia.org/wiki/File:KB_Eng-Rus_QWERTY(\%D0\%99\%D0\%A6\%D0\%A3\%D0\%9A\%D0\%95\%D0\%9D).svg, „KB Eng-Rus QWERTY(ЙЦУКEH)“, https://creativecommons.org/licenses/by-sa/4.0/legalcode)

Die gescheiterte Frage (\#3), die sich auf die Seitenanzahl der weitergeleiteten Datei bezieht, wird durch die in Nachricht \#4 übermittelte Mitteilung ähnlichen Inhalts ersetzt, welche in kyrillischer Schrift verfasst und von $A$ anschließend bestätigt wird. Das in (89) vorliegende Problem tritt in der Schreibroutine russischer PC- und Laptop-Nutzer so regelmäßig auf, dass viele Internetbrowser inzwischen im Stande sind, viele mit lateinischen Buchstaben eingegebene Suchbegriffe zu erkennen und automatisch eine Alternative vorzuschlagen (vgl. Abb. 3):

\section{kbyudbcbrf | \\ лингвистика \\ лингвистика бг \\ лингвистика текста}

@ Q

Abb. 3: Screenshot einer Suchanfrage bei Google, in der der fälschlicherweise mit lateinischen Buchstaben eingegebene Suchbegriff лингвистика (,Linguistik') automatisch erkannt wird

Die WhatsApp-Desktop-Version dagegen scheint eine solche Funktion nicht anzubieten: Es konnte zumindest weder eine entsprechende Option im Menü 
der Desktop-Version selbst noch eine Information darüber auf der Serviceseite von WhatsApp gefunden werden.

\section{Interpunktionszeichen}

Vier Belege in den russischen und 18 in den deutschen Kurznachrichtendialogen lassen sich als (ortho)graphische Korrekturen einordnen, bei denen ein fehlendes Interpunktionszeichen nachträglich insertiert oder ein falsch gesetztes Zeichen durch ein anderes substituiert wird. Dabei fungiert in allen Fällen mit Ausnahme von (90) das Fragezeichen als Reparans, das in den russischen Daten ausschließlich bei Insertionen und in den deutschen sowohl bei Insertionen (elf Fälle) als auch bei Substitutionen (sechs Fälle) vorkommt. Im Falle von Substitutionen treten Interpunktionszeichen wie Punkt oder Komma, Einzelbuchstaben und Zahlen als Reparanda auf. Im einzigen Beispiel im Korpus, in dem ein Ausrufezeichen als Reparans fungiert, stellt ein Buchstabe die Problemquelle dar:

(90) Auszug aus dem WhatsApp-Dialog \#4070:

Schreiberin $A$

Schreiberin $B$

\begin{tabular}{|c|}
\hline Hat VORNAME dir schon geschrieben? \\
\hline Nachricht \#1 - 17.03.2016 - 09:40 \\
\hline
\end{tabular}

\begin{tabular}{|c|}
\hline Jaa eben \\
\hline Nachricht \#2 - 17.03.2016 - 09:42 \\
\hline
\end{tabular}

\begin{tabular}{|c|}
\hline Sie sind zusammen :D \\
\hline Nachricht \#3 - 17.03.2016-09:42 \\
\hline
\end{tabular}

\begin{tabular}{|c|}
\hline Nein!!!!! \\
\hline Nachricht \#4 - 17.03.2016 - 09:43 \\
\hline
\end{tabular}

\begin{tabular}{|c|}
\hline Omg \\
\hline Nachricht \#5 - 17.03.2016-09:43 \\
\hline
\end{tabular}

\begin{tabular}{|c|}
\hline Doch $\Theta$ \\
\hline Nachricht \#6 $-17.03 .2016-09: 44$ \\
\hline
\end{tabular}

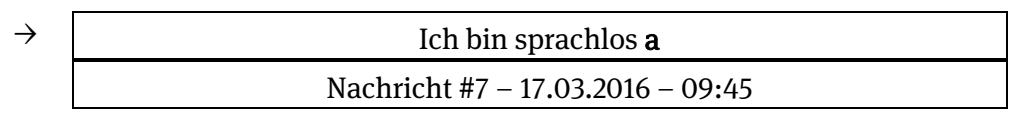


$\rightarrow$

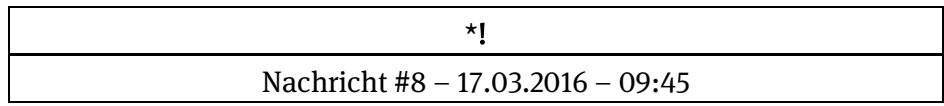

\begin{tabular}{|c|}
\hline :D :D \\
\hline Nachricht \#9 $-17.03 .2016-10: 01$ \\
\hline
\end{tabular}

Beim Lästern über ein Pärchen äußert Schreiberin $A$ in \#7 ihre Verblüffung bezüglich der Tatsache, dass die beiden Personen zueinander gefunden haben. Diese Bewertung schließt mit einem „a“, welches man u.U. als den Anfang eines nächsten Wortes interpretieren könnte. Erst die in \#8 durchgeführte Selbstreparatur legt offen, dass anstelle des ,abgebrochenen“ „a“ ein Ausrufezeichen stehen soll. Das als Reparans fungierende Ausrufezeichen wird hier mit einem vorangestellten Asterisk markiert (zu Funktionen von Asterisken als Reparaturmarker vgl. Kap. 3.4.2.1 sowie Mostovaia 2018).

Während in (90) eine Substitution als Reparaturoperation vorliegt, wird anhand von (91) eine Insertion veranschaulicht:

(91) Auszug aus dem WhatsApp-Dialog \#4038

Schreiberin $A$

Schreiber $B$

\begin{tabular}{|c|}
\hline Dann können wir ja gehen \\
\hline Nachricht \#6 - 04.04.2016 - 13:58:00 \\
\hline
\end{tabular}

\begin{tabular}{|l|}
\hline Können dann ja direkt was einkaufen \\
\hline Nachricht \#7 - 04.04.2016 - 13:59:00 \\
\hline
\end{tabular}

\begin{tabular}{|c|}
\hline Ich trinke b Kaffee dann komme ich \\
\hline Nachricht \#8 - 04.04.2016 - 13:59:00 \\
\hline
\end{tabular}

$\rightarrow$

\begin{tabular}{|c|}
\hline Ok schatz \\
\hline Nachricht \#9 $-04.04 .2016-13: 59: 00$ \\
\hline
\end{tabular}

$\rightarrow$

\begin{tabular}{|c|}
\hline $\boldsymbol{?}$ \\
\hline Nachricht \#10 - 04.04.2016-13:59:00 \\
\hline
\end{tabular}

Jap alles klar Schatz

Nachricht \#11 - 04.04.2016 - 13:59:00

Im vorherigen Verlauf des WhatsApp-Dialogs schlägt Schreiberin $A$ ihrem Ehemann (Schreiber $B$ ) vor, einen Spaziergang mit ihrem Hund in einem Wald zu 
machen (nicht abgebildet). Schreiber $B$ stimmt dem Vorschlag zu und teilt in \#8 mit, dass er nach Hause kommen wird, nachdem er seinen Kaffee getrunken hat. Die Problemquelle liegt dabei in Nachricht \#9, welche wegen des fehlenden Satzzeichens als eine Bestätigung auf eine der vorherigen Nachrichten von $A$ interpretiert werden kann, z.B. als eine Zustimmung zu dem Vorschlag, den Spaziergang mit dem Einkaufen zu verbinden (vgl. \#7). Allerdings zeigt das nachgelieferte Fragezeichen in \#10, dass $B$ nicht seine Zustimmung zum Ausdruck bringt, sondern das Einverständnis von $A$ damit einholen will, und zwar dafür, dass er nicht direkt nach Hause geht, sondern zuerst einen Kaffee trinkt. Interaktional gesehen wären das zwei unterschiedliche sprachliche Handlungen, sodass die (ortho)graphische Korrektur in dem Fall gleichzeitig auch eine pragmatische Funktion ausübt (vgl. Pfeiffer 2015: 54). ${ }^{99}$

\section{Einzelne Wörter}

Neben den (ausbleibenden) Interpunktionszeichen werden im Rahmen von (ortho)graphischen Reparaturen einzelne Lexeme behandelt, deren Schreibung korrigiert wird. Dabei kann allerdings nicht immer unterschieden werden, ob das vorliegende Reparandum jeweils durch einen Vertipper oder einen unerwünschten Eingriff des Autokorrekturprogrammes ausgelöst wird, weil das Autokorrekturprogramm auch fehlerhafte Vorschläge anbieten kann, die einem Tippfehler ähneln. Daher wird diese Unterscheidung nur in den Fällen getroffen, in denen beispielsweise auf die Gründe des entstandenen Reparandums metasprachlich eingegangen wird (vgl. (122a-d)). In Beispielen wie (92), in denen beide Optionen möglich sind, wird dagegen darauf verzichtet:

(92) Auszug aus dem WhatsApp-Dialog \#3570:

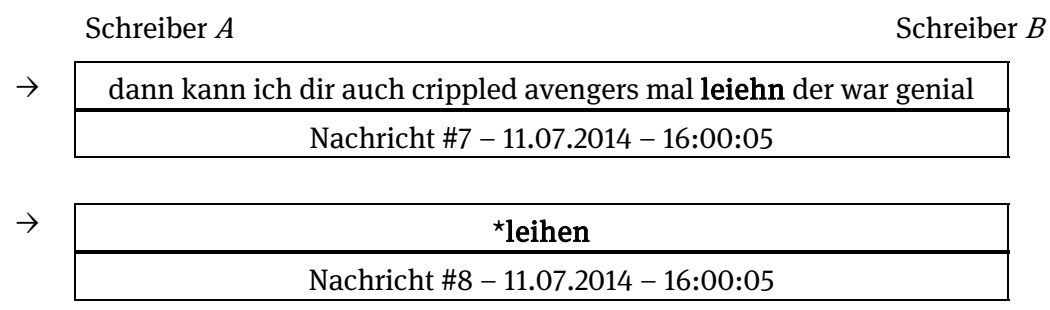

99 Auch weitere Reparanda aus den untersuchten Daten lassen sich mehr als einem Reparandatyp zuordnen. Aufgrund dieser Mehrfachzuordnungen übersteigt die Summe aller in Kap. 6.1.1.1 beschriebenen Reparanda die Gesamtanzahl der analysierten selbstinitiierten Selbstreparaturen in next opportunity space. 


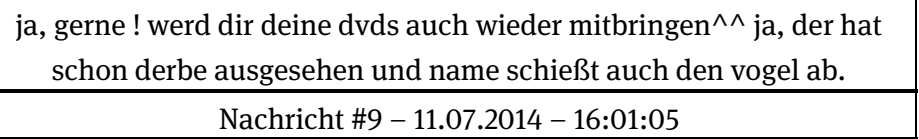

Zwei Freunde, Schreiber $A$ und Schreiber $B$, machen im vorherigen Verlauf des Dialogs (92) einen Termin für $B$ 's Besuch aus. Nachdem der Termin steht, kündigt Schreiber $A$ an, dass er vorhat, seinem Freund $B$ eine DVD mit dem Film Crippled Avengers zu leihen, der $A$ 's Meinung nach sehr empfehlenswert ist. Allerdings enthält das von $A$ in \#7 eingetippte Verb „leiehn“ einen Buchstabendreher, der direkt in der darauffolgenden Nachricht durch das Verschicken der orthografisch korrekten Version mit dem vorangestellten Asterisk korrigiert wird.

Während sich drei Reparaturen in den russischen und 22 in den deutschen Daten eindeutig den (ortho)graphischen Korrekturen zuordnen lassen, in deren Rahmen einzelne Wörter als Reparanda fungieren, findet sich in dem jeweiligen Subkorpus (neun Fälle im russischen und 38 im deutschen) zudem eine weitere Gruppe von Korrekturen, bei denen das Reparans und das Reparandum auf graphischer Ebene einander zwar mehr oder weniger ähneln, aber zwei unterschiedliche Wörter darstellen. Dabei handelt es sich z.B. um Substitutionen wie Bein - Nein (vgl. (101)), dies - fies (vgl. (122d)) sowie надо (,man muss/es ist nötig‘) - нас (,uns`) (vgl. (103)). Im Zusammenhang mit solchen Fällen stellt sich also die Frage, wo die Grenze zwischen (ortho)graphischen und lexiko-semantischen Problemquellen zu ziehen ist. In der vorliegenden Arbeit werden solche Reparanda als eine Sondergruppe behandelt, die an der Grenze zwischen (ortho)graphischen und lexiko-semantischen Reparaturen anzusiedeln ist.

\section{Emoticons}

Die im deutschen Subkorpus durchgeführten (ortho)graphischen Korrekturen beziehen sich nicht nur auf die Interpunktionszeichen sowie einzelne Lexeme, sondern in zwei Fällen auch auf Emoticons (vgl. auch Mostovaia 2018: 57-58), wobei diese je im Rahmen einer Substitution (vgl. (93)) und einer Insertion (vgl. (94)) bearbeitet werden:

(93) Auszug aus dem Dialog \#2061:

Schreiberin $A$

Schreiberin $B$

\begin{tabular}{|c|}
\hline Okay dann machen wir das einfach die Woche danach $:^{\star}$ \\
\hline Nachricht \#6-08.02.2013-07:34:30 \\
\hline
\end{tabular}




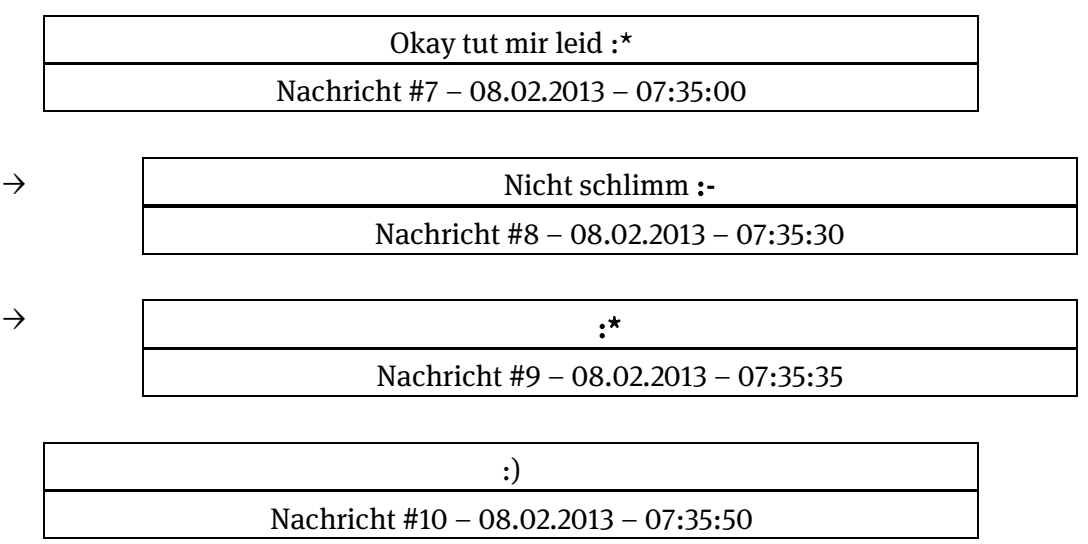

In (93) handelt es sich um eine gescheiterte Verabredungssequenz, die von Schreiberin $A$ mit dem Vorschlag, am Sonntag frühstücken zu gehen, initiiert wurde. Nachdem dieser Vorschlag von $B$ angenommen wurde, zieht $A$ ihn wieder zurück, weil ihr der Termin doch nicht passt (vgl. \#1-5, nicht abgebildet). Daraufhin schlägt $B$ vor, das gemeinsame Frühstück auf die nächste Woche zu verschieben, was bei $A$ auf Zustimmung stößt. Die Tatsache, dass Schreiberin $A$ die Verabredungssequenz initiiert und dann wieder abgebrochen hat, führt dazu, dass $A$ sich in \#7 entschuldigt. $B$ akzeptiert die Entschuldigung und stuft das Problem herab. Dabei beendet sie ihre Nachricht mit dem Emoticon ,:-“, welches allerdings in der Form nicht existiert. Anschließend wird der Bindestrich im Reparandum ,:-“ durch einen Asterisk ersetzt, sodass sich daraus ein ,Kuss'-Emoticon „:*“ ergibt, dass phatische Kommunikation markiert.

Bei dem in (94) vorliegenden Auszug handelt es sich ebenfalls um eine Verabredungssequenz, in der die Zeit des Treffens ausgehandelt wird:

(94) Auszug aus dem WhatsApp-Dialog \#3396:

Schreiberin $A$

Schreiberin $B$

\begin{tabular}{|c|}
\hline Heyho! Hast du dir schon eine Uhrzeit ausgedacht? :) \\
\hline Nachricht \#1 - 25.04.2014 - 12:14:05 \\
\hline
\end{tabular}

$14 \mathrm{Uhr} ?$

Nachricht \#2 - 25.04.2014 - 13:38:05

\begin{tabular}{|c|}
\hline Okay geht klar \\
\hline Nachricht \#3 $-25.04 .2014-13: 43: 05$ \\
\hline
\end{tabular}




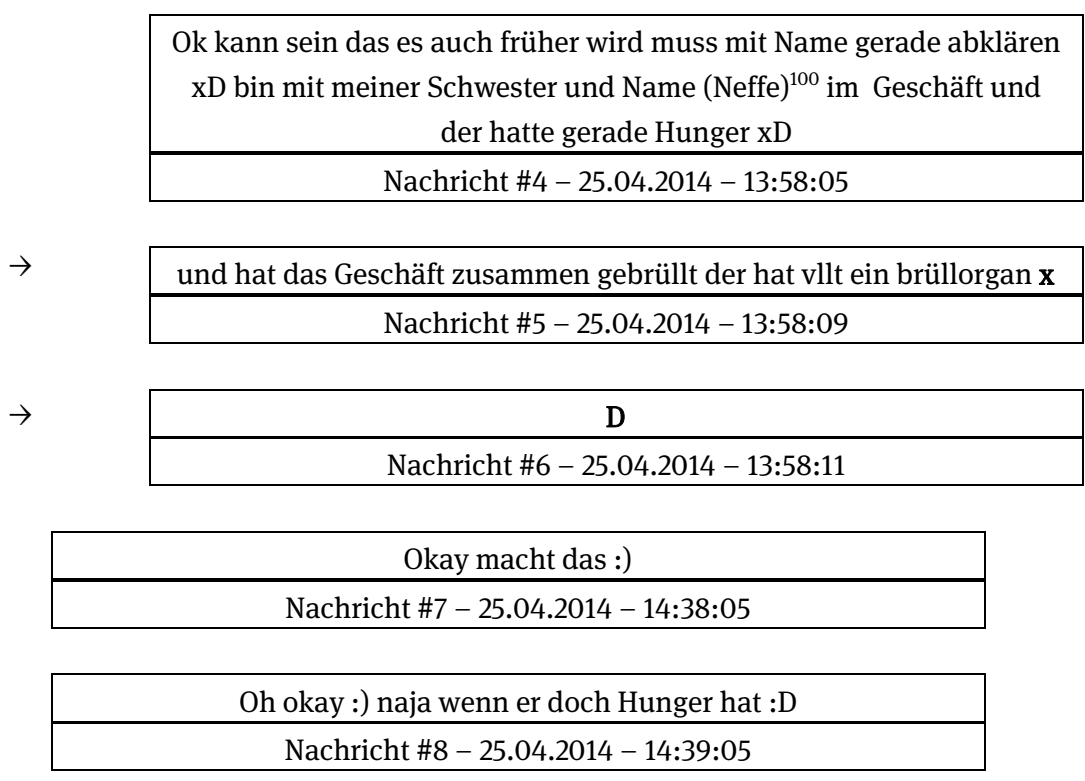

Nachdem $B$ ihren Zeitvorschlag in \#2 geäußert hat, der von $A$ anschließend bestätigt wird, relativiert sie den von ihr vorher genannten Zeitpunkt und geht zu einer Mitteilung über ihren aktuellen Standort über (\#4). Dabei berichtet sie über das Verhalten ihres Neffen (vgl. \#4-5) und versieht ihre Nachrichten mit dem Emoticon „xD“, bei dem das $x$ zusammengekniffene Augen und das $D$ den weit geöffneten lachenden Mund symbolisieren sollen und welchem man in Anlehnung an Pappert (2017) eine Kommentar- bzw. Bewertungsfunktion zuschreiben kann. Allerdings wird in Nachricht \#5 nur die erste Konstituente des Emoticons verschickt, was eine selbstinitiierte Selbstreparatur in \#6 nach sich zieht. Diese wird durch das Verschicken der zweiten Konstituente des Emoticons (,D“) realisiert, welche in die ursprüngliche Nachricht insertiert werden soll.

\section{(ii) Lexiko-semantische Reparanda}

Im Gegensatz zu (ortho)graphischen Reparaturen handelt es sich bei lexiko-semantischen Reparaturen um solche, in denen die problematische Denotation

100 Die in der MoCoDa zur Verfügung stehenden Nachrichten werden von Spendern selbst anonymisiert. Dabei wird auf unterschiedliche Strategien zurückgegriffen. Eine davon besteht darin, dass alle Personennamen durch die Angabe Name ersetzt werden, wie in Nachricht \#4 aus (94). Dabei ist es allerdings unklar, ob die Angabe in Klammern „Neffe“ aus der Originalnachricht stammt oder im Laufe der Anonymisierung hinzugefügt wurde. 
eines sprachlichen Ausdrucks bearbeitet wird (sieben Fälle in den deutschen und acht in den russischen Daten). Zu solchen Fällen kann u.a. die in (95) vorliegende Reparatur gezählt werden:

(95) Auszug aus dem WhatsApp-Dialog \#3643:

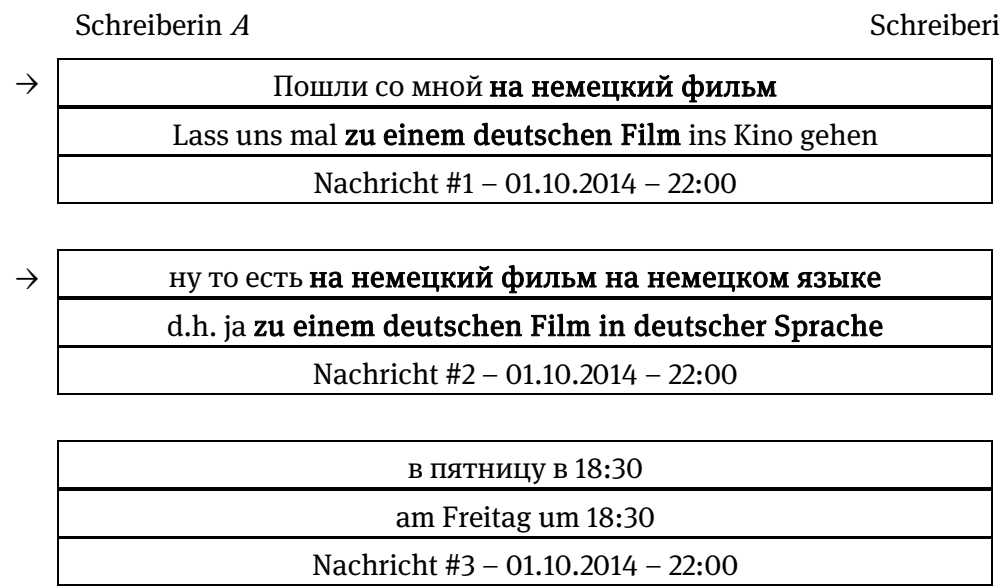

\begin{tabular}{|c|}
\hline О, ну здорово, и что я там буду делать? \\
\hline Oh, das ist zwar toll, aber was soll ich da tun? \\
\hline Nachricht \#4-01.10.2014 - 22:01 \\
\hline
\end{tabular}

\begin{tabular}{|c|}
\hline Будешь смотреть со мной фильм. Да пошлиии, ну пожаааалуйста! \\
\hline $\begin{array}{c}\text { Du wirst dir mit mir zusammen den Film anschauen. Lass uns doch } \\
\text { hingehen, biiitte! }\end{array}$ \\
\hline Nachricht \#5 - 01.10.2014 - 22:02 \\
\hline
\end{tabular}

Die in (95) abgebildete selbstinitiierte Selbstreparatur wird mit einer Kombination aus der Partikel „ну“ (,nun, halt, ja') und der Konjunktion „то есть“ (,das heißt') eingeleitet. In \#1 unterbreitet Schreiberin $A$ ihrer Freundin einen Vorschlag, sich im Kino einen deutschen Film anzuschauen. Die Nominalphrase „немецкий фильм“ (,ein deutscher Film`) würde man im Russischen in dem Kontext zunächst eher als ,ein von einem deutschen Regisseur gedrehten Film` interpretieren. Dabei setzt man i.d.R. implizit voraus, dass dieser Film synchronisiert wurde und daher in russischer Sprache gezeigt wird. Wie es sich aber in \#2 herausstellt, bezieht sich $A$ als Germanistikstudentin mit „немецкий фильм“ (,ein deutscher Film') auf einen deutschen Film, welcher in Originalsprache (also Deutsch) gezeigt wird. Die von ihr gemeinte Bedeutung der Nominalphase „немецкий фильм“ (,ein deutscher Film‘) expliziert $A$ für ihre Freun- 
din, die Management studiert und mit der deutschen Sprache daher nichts zu tun hat, durch die Insertion des Präpositionalattributs „на немецком языке“ (,in deutscher Sprache').

\section{(iii) Morphosyntaktische Reparanda}

Im gesamten Korpus sind Reparanda, die man der morphosyntaktischen Ebene zuordnen könnte, lediglich mit fünf Fällen in den russischen und zwölf in den deutschen Daten vertreten. Dabei handelt es sich in erster Linie im Gegensatz zum Splitting im Sinne von Beißwenger (2007) um nicht intendiert abgebrochene Nachrichten, die in der Folgenachricht fortgesetzt werden, wie in (96):

(96) Auszug aus dem Dialog \#1320:

Schreiberin $A$

Schreiber $B$

\begin{tabular}{|c|}
\hline Hey, wie war deine Shoppingtour gestern? Liebe Grüße! \\
\hline Nachricht \#1 - 14.10.2011 - 16:57:00 \\
\hline
\end{tabular}

\begin{tabular}{|c|}
\hline $\begin{array}{l}\text { Nicht sehr erfolgreich, hätte bei dem ein oder andern pulli dei } \\
\text { kompetente Meinung dringend nötig gehabt. Wie war Rücken? }\end{array}$ \\
\hline Nachricht \#2 - 14.10.2011 - 17:00:00 \\
\hline $\begin{array}{l}\text { Alle Sorge war umsonst! Lief super und nun ich hab sogar } \\
\text { Muskelkater!:-)Bei der nächsten Shoppingtour }\end{array}$ \\
\hline Nachricht \#3 - 14.10.2011 - 17:06:00 \\
\hline Bin ich wieder dabei!:-) \\
\hline Nachricht \#4 - 14.10.2011 - 17:06:05 \\
\hline Das iPhone ist zu schnell für mich! :-)lg \\
\hline Nachricht \#5 - 14.10.2011 - 17:06:10 \\
\hline
\end{tabular}

Alles klar, dann weiß ich ja an wen ich mich bei Rückenschmerzen demnächst wenden muss. Müssen dann bald mal nen Date ausmachen, brauche dringend ne neue Hose

Nachricht \#6 - 14.10.2011 - 17:12:00

In (96) steigt Schreiberin $A$ mit der Frage nach $B$ 's „Shoppingtour“ (\#1) ein, bei der sie ihn nicht begleiten konnte, da sie an dem Tag einen Rückengymnastikkurs geben musste. Seine „Shoppingtour“ bewertet $B$ als „Nicht sehr erfolgreich“ (\#2), was er mit $A$ 's Abwesenheit in Verbindung bringt. Daraufhin gibt $A$ 
ihm ein Versprechen, nächstes Mal mitzugehen, wobei sie den ersten Teil dieses Versprechens in \#3 („Bei der nächsten Shoppingtour“) und den zweiten in \#4 („Bin ich wieder dabei!:-)“) verschickt. Zum dadurch entstandenen Nachrichtenabbruch gibt $A$ in \#5 einen expliziten Metakommentar ab, in dem sie erklärt, dass dieser von ihr nicht absichtlich erzeugt wurde, sondern beim Eintippen der Nachricht auf ihrem iPhone zufällig zustande kam.

\section{(iv) Pragmatische Reparanda}

Im Rahmen von pragmatischen Reparaturen (42 im deutschen und elf im russischen Subkorpus) werden unterschiedliche Problemquellen bearbeitet. Dabei betreffen diese Reparaturen hauptsächlich entweder eine problematische Referenz (97) oder die in einer Nachricht zum Ausdruck gebrachte sprachliche Handlung, die vom Produzenten der Problemquelle modifiziert (98) oder zurückgenommen (99) wird (vgl. dazu Reparaturen des Handlungsformats bei Pfeifer 2015: 81-83).

Bei problematischen Referenzen werden meistens deiktische Ausdrücke bearbeitet, mit denen auf bestimmte Zeitpunkte bzw. -abschnitte, Orte, Personen und Objekte verwiesen wird:

(97) Auszug aus dem WhatsApp-Dialog \#4250:

\begin{tabular}{|c|c|}
\hline & Schreiberin $A$ \\
\hline & Wie wars? \\
\hline & Nachricht \#1 - 10.12.2016 - 16:40 \\
\hline & also nich das duschen :D \\
\hline & Nachricht \#2 - 10.12.2016 - 16:40 \\
\hline
\end{tabular}

Schön und warm und nass :D Das Spiel war kalt und nass und nicht so spannend...

Nachricht \#3 - 10.12.2016 - 16:41

\begin{tabular}{|c|}
\hline Oh nooo \\
\hline Nachricht \#4-10.12.2016-16:41 \\
\hline
\end{tabular}

Mit dem Enklitikon $s$ aus der Klitisierung „wars“ (\#1) wird anaphorisch auf eine Handlung verwiesen, die entweder im vorherigen, allerdings in der Mobile Communication Database nicht zur Verfügung gestellten, Verlauf des WhatsApp-Dialogs oder im Rahmen einer mündlichen Kommunikation thematisiert wurde. Die in \#2 durchgeführte Reparatur sowie die in \#3 erfolgte Reaktion 
von $B$ lassen jedoch darauf schließen, dass sich das $s$ theoretisch entweder auf das Duschen oder das Spiel beziehen könnte, wobei das gemeinte Bezugsnomen von $A$ durch das Ausschließen der nicht-gemeinten Referenz in \#2 präzisiert wird. Dass ein potenzielles Referenzproblem vorlag, kann man an der scherzhaften ,falschen' Antwort von $B$ sehen. Zugleich zeigt seine im Anschluss gelieferte ,richtige' Antwort an, dass die Referenzreparatur erfolgreich war.

Die gesamte, in einer Nachricht zum Ausdruck gebrachte sprachliche Handlung wird u.a. im folgenden Auszug aus einem Dialog zwischen Schreiberin $A$ und ihrer Mutter (Schreiberin $B$ ) modifiziert. Hier erzeugt der in \#2 nachgetragene Bedingungssatz „wenn das geht“, der mit einem präpositionierten Reparaturmarker „Also“ und einem postpositionierten „heißt das“ (zu das heißt als „Reformulierungsindikator“ vgl. Kaiser 2016) gerahmt wird, den Wechsel von einer Mitteilung zu einer Nachfrage, die eine Erlaubnis seitens der Mutter interaktional erforderlich und relevant macht:

(98) Auszug aus dem WhatsApp-Dialog \#4157:

Schreiberin $A$ Schreiberin $B$

\begin{tabular}{l}
$\rightarrow \quad$ Ganz vergessen dir zu sagen, dass ich morgen abend komme! \\
\hline Nachricht \#1-12.07.2016 - 22:00 \\
$\rightarrow \quad$ Also wenn das geht heißt das \\
\hline Nachricht \#2 - 12.07.2016-22:00
\end{tabular}

\begin{tabular}{|c|}
\hline Klar doch. \\
\hline Nachricht \#3-12.07.2016-22:06 \\
\hline
\end{tabular}

Des Weiteren können zu pragmatischen Reparaturen auch einige Fälle gezählt werden, in denen eine Ablehnung bzw. der Ausdruck des NichtEinverständnisses durch eine Zusage oder eine Zustimmung innerhalb eines kurzen Zeitraums abgelöst wird (vgl. (101)). Eine somit erfolgte Substitution einer sprachlichen Handlung durch eine andere wird in den analysierten Daten vereinzelt dadurch eingesetzt, um eine scherzhafte Interaktionsmodalität zu erzeugen bzw. eine Frotzelaktivität im Sinne von Günthner $(1996,1999)$ zu gestalten (vgl. dafür (118)).

Darüber hinaus lässt sich im Untersuchungskorpus eine Gruppe von pragmatischen Reparaturen beobachten, bei denen eine sprachliche Handlung z.B. eine Informationsanfrage (vgl. \#1285 in der MoCoDa) - zurückgenommen bzw. außer Kraft gesetzt wird, da diese ihre Relevanz für die Kommunikation verloren hat. Als spezielle Untergruppe solcher pragmatischen Reparaturen 
können diejenigen Fälle angesehen werden, bei denen eine Nachricht an den falschen Adressaten verschickt wird. Die analysierten Daten weisen je einen Fall in den deutschen und den russischen Daten auf, die strukturell ähnlich aufgebaut sind. Daher wird hier nur einer der beiden Belege präsentiert:

(99) SMS-Dialog \#2910:

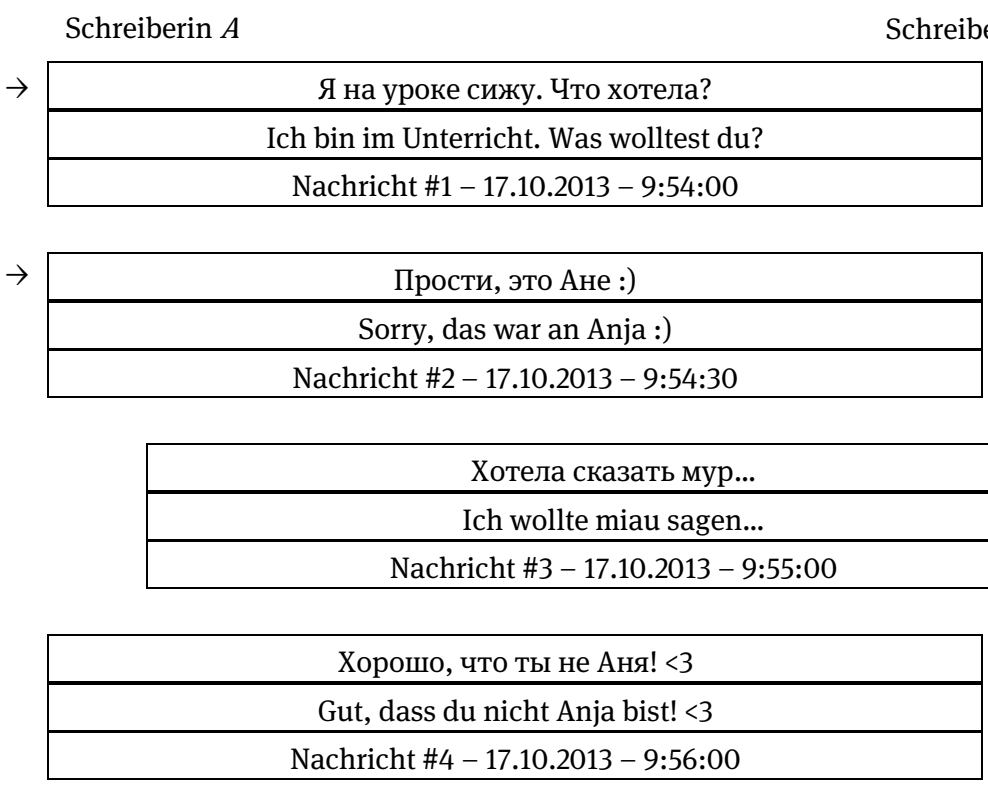

Der in (99) abgebildeten Nachricht \#1 aus dem russischen Subkorpus ist ein Anruf der Kommilitonin von Schreiberin $A$ vorausgegangen, den sie aber nicht annehmen konnte, da sie in jenem Moment im Rahmen ihres Schulpraktikums bei einem Unterricht hospitierte. Auf den erhaltenen Anruf reagiert $A$ jedoch mit einer SMS-Nachricht, in der sie die Nicht-Annahme des Anrufs rechtfertigt und ihre Kommilitonin nach dem Grund für den Anruf fragt. Diese Nachricht schickt sie aber versehentlich nicht an die Kommilitonin, sondern an ihren festen Freund, Schreiber $B$. Dabei ist Nachricht \#1 so formuliert, dass sie sich durch die Verbform mit dem Femininum-Suffix -а (хотел- - -,wolltest-FEM`) nur an eine weibliche Person richten kann. Würde $A$ ihren Freund fragen Was wolltest du?, hätte sie хотел- $\varnothing$ (,wolltest-MASK') schreiben müssen. Die darauffolgende explizite Erklärung in \#2, von $A$ als präventive Maßnahme zur Aufrechterhaltung der gegenseitigen Verständigung eingesetzt, besteht aus zwei Komponenten: aus der Entschuldigungsfloskel „Прости“ (,Sorry/Entschuldige mich/Verzeih mir‘; vgl. repair prefaces bei Kitzinger 2013: 240-241) sowie aus der Klarstellung des Rezipienten, die in dem Fall durch die Benennung des richtigen Adressaten 
realisiert wird. Dennoch geht Schreiber $B$ nicht auf die Reparatur selbst, sondern auf die Problemquelle in \#1 ein, indem er diese als Anlass für seine scherzhafte Antwort in \#3 nutzt und dabei das Verb „Хотела“ (,wollte-FEM“) für die Selbstreferenz wiederaufnimmt. ${ }^{101}$

Im Zusammenhang mit den sprachlichen Aktivitäten, die in der Folgenachricht zurückgenommen werden, soll auf das reparative Potenzial der beim Schreiben via WhatsApp und Viber gegebenen Möglichkeit hingewiesen werden, eine bereits abgeschickte Nachricht nachträglich, und zwar im Idealfall, bevor sie vom Rezipienten gelesen wird, zu löschen. Die gelöschte Nachricht verschwindet allerdings aus dem Nachrichtenverlauf nicht komplett, sondern wird durch die jeweilige in Abb. 4 dargestellte Systemnachricht ersetzt.

Gegenüber
Q Diese Nachricht wurde gelöscht.

14:46

Q Du hast diese Nachricht gelöscht.
Gegenüber

Q Данное сообщение удалено

$14: 46$

Q Вы удалили данное сообщение $14: 47$

Abb. 4: Anzeige einer gelöschten Nachricht in einem WhatsApp-Dialog: jeweils die erste Nachricht - Ansicht aus der Perspektive des Rezipienten der gelöschten Nachricht, jeweils die zweite Nachricht - Ansicht aus der Perspektive des Produzenten der gelöschten Nachricht

Bis jetzt liegen m.W. keine Studien vor, die sich mit interaktionalen Funktionen der Nachrichten auseinandersetzen, die von Schreibern gelöscht wurden, bzw. mit Kontexten, in denen von dieser Möglichkeit Gebrauch gemacht wird. Allerdings kann aufgrund persönlicher Beobachtungen des Schreibverhaltens einzelner russischen WhatsApp-Nutzer festgestellt werden, dass das Löschen von

101 Die vergleichbare Reparatur aus den deutschen Daten „Sorry war nicht an dich“ (vgl. \#2146) setzt sich ebenso aus zwei Komponenten zusammen - aus einer Entschuldigungsfloskel sowie einem Verweis darauf, dass die Nachricht nicht für die Rezipientin gedacht war. Neben diesem Beleg kommt die Entschuldigungsfloskel sorry im Kontext einer selbstinitiierten Selbstreparatur in zwei weiteren Dialogen aus dem deutschsprachigen Subkorpus der MoCoDa vor (vgl. \#3828 und \#4110). Das russische Subkorpus weist lediglich eine einzige selbstinitiierte Selbstreparatur mit einer Entschuldigungsfloskel auf, nämlich diejenige, die in (99) vorgestellt wurde. Die Entschuldigungsfloskel sorry wird sowohl von Levelt (1983) als auch Lerner/Kitzinger (2015: 59) im Rahmen von selbstinitiierten Selbstreparaturen als eine Art Reparaturinitiierung aufgefasst, wobei Levelt (1983) darauf mit „editing term“ und Lerner/Kitzinger (2015: 59) mit „repair preface“ referieren (zu unspezifischen Fremdinitiierungen mit sorry im Kontext von fremdinitiierten Selbstreparaturen vgl. Kap. 2.3.1.1). 
Nachrichten durchaus zu Reparaturzwecken ${ }^{102}$ eingesetzt werden kann. Dabei wird auf diese Strategie nicht nur dann zurückgegriffen, wenn die Nachricht an den falschen Adressaten geschickt wurde, sondern - wie in (100) - auch dann, wenn in der gelöschten Nachricht ein (ortho)graphisches Reparandum vorliegt:

(100) Auszug aus den für die russische Version der MoCoDa 2 erhobenen Daten (Löschen einer WhatsApp-Nachricht im Kontext einer selbstinitiierten Selbstreparatur):

Q Данное сообщение удалено

20:34

Оу, ну тогда да $20: 34$
1 A: Diese Nachricht wurde gelöscht.*

2 A: oh na dann ist es okay

*Die gelöschte Nachricht lautet: „Оу, ну тогда она“(,Oh, na dann ist es sie`).

Der in (100) dargestellte Auszug ist einem WhatsApp-Dialog zwischen zwei Freundinnen entnommen, in dem sich $B$ über hohe Ticketpreise für das Konzert einer Band beschwert. Schreiberin $A$ stimmt ihr zu und merkt an, dass solche Preise für die Band dieser ,Klasse' viel zu hoch sind. Das bringt $B$ dazu, ihre vorherige Nachricht $\mathrm{zu}$ relativieren und offenzulegen, dass sich der von ihr genannte Preis auf die letzten und somit die teuersten Tickets bezieht und es auch günstigere Tickets gab. Darauf reagiert Schreiberin $A$ mit Nachricht \#1 aus (100), die sie jedoch direkt danach löscht, um stattdessen Nachricht \#2 zu verschicken. Es ist mir allerdings gelungen, den Inhalt der gelöschten Nachricht noch rechtzeitig zu erfassen, was leider bei der Datenerhebung ausschließlich durch den Einsatz einer speziellen Software ermöglicht werden kann, die alle Bildschirmaktivitäten - und zwar im Idealfall aller an der Interaktion beteiligten Schreiber - mitaufzeichnet. Für die Datensammlung mittels der MoCoDa ist dies nicht möglich, weil dort lediglich Dialoge als ,fertige Produkte“ hochgeladen werden können. Die gelöschte Nachricht lautete „Оу, ну тогда она“ (,Oh, na dann ist es sie') und enthielt das Reparandum „она“ (,sie'), welches in der direkt danach verschickten Nachricht durch das Responsiv „да“ (,ja') ersetzt wird. Der Rest der Nachricht entspricht exakt dem Wortlaut des gelöschten Beitrags.

102 Selbst auf der Internetseite von WhatsApp werden Nutzer darauf aufmerksam gemacht, dass sie mit dem Löschen einer Nachricht im Grunde genommen eine selbstinitiierte Selbstreparatur durchführen können: „Das ist vor allem dann sinnvoll, wenn du eine Nachricht aus Versehen im falschen Chat gepostet hast oder die Nachricht einen Fehler enthält.“ (https://faq.whatsapp.com/de/android/26000068/) 


\subsubsection{Reparaturinitiierungen}

Im Folgenden wird zunächst auf selbstinitiierte Selbstreparaturen im next opportunity space eingegangen, die ohne explizite Reparaturmarker realisiert werden. Danach werden drei Gruppen von expliziten Reparaturinitiierungen vorgestellt, die in den analysierten Daten vertreten sind. Das sind nonverbale graphische Reparaturinitiierungen, lexikalische Reparaturmarker sowie phrasale und satzwertige Reparaturinitiierungen. Anschließend wird kurz veranschaulicht, wie explizite Reparaturmarker miteinander kombiniert werden können.

\section{(i) Reparaturen ohne explizite Reparaturmarker}

In 50\% der selbstinitiierten Selbstreparaturen im next opportunity space im deutschen und 53\% im russischen Korpus kommen keine expliziten Reparaturmarker vor (jeweils 69 und 52 Fälle). Dabei kann man in den meisten solcher Fälle in Anlehnung an Pfeiffer (2015) von impliziten Reparaturmarkern in Form einer Retraktion ausgehen (vgl. Beispiel (12) aus der gesprochenen Sprache). Sowohl in den russischen als auch in den deutschen Daten bleiben explizite Reparaturmarker in erster Linie bei Korrekturen aus, die ein (ortho)graphisches Reparandum bearbeiten (35 Fälle in den deutschen und sieben in den russischen Dialogen; dazu kommen jeweils 17 und drei Belege, die als Sondergruppe an der Grenze zwischen (ortho)graphischen und lexiko-semantischen Reparaturen anzusiedeln sind). Ohne explizite Reparaturmarker werden in den analysierten Daten auch lexiko-semantische, morphosyntaktische sowie pragmatische selbstinitiierten Selbstreparaturen im next opportunity space realisiert. ${ }^{103}$ Auszug (101) stellt einen Fall dar, in dem explizite Reparaturmarker sowohl bei der (ortho)graphischen Korrektur (\#3) als auch bei der sich daran anschließenden pragmatischen Reparatur (\#4) ausbleiben:

(101) Dialog \#2215:

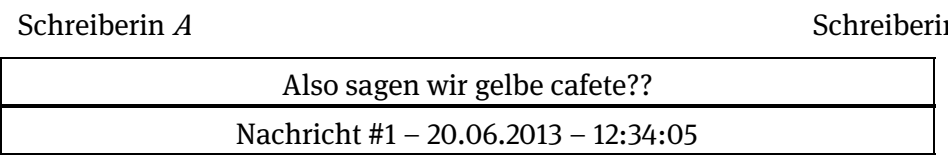

103 Im deutschen Subkorpus liegen vier lexiko-semantische, sieben morphosyntaktische und zehn pragmatische selbstinitiierte Selbstreparaturen im next opportunity space ohne explizite Reparaturmarker vor. Das russische Subkorpus weist drei lexiko-semantische, drei morphosyntaktische und fünf pragmatische Reparaturen ohne explizite Reparaturmarker auf. An dieser Stelle muss allerdings darauf hingewiesen werden, dass die in diesem Unterkapitel genannten Reparandatypen in einem russischen und in vier deutschen Belegen jeweils zwei Problemtypen zugeordnet wurden. 


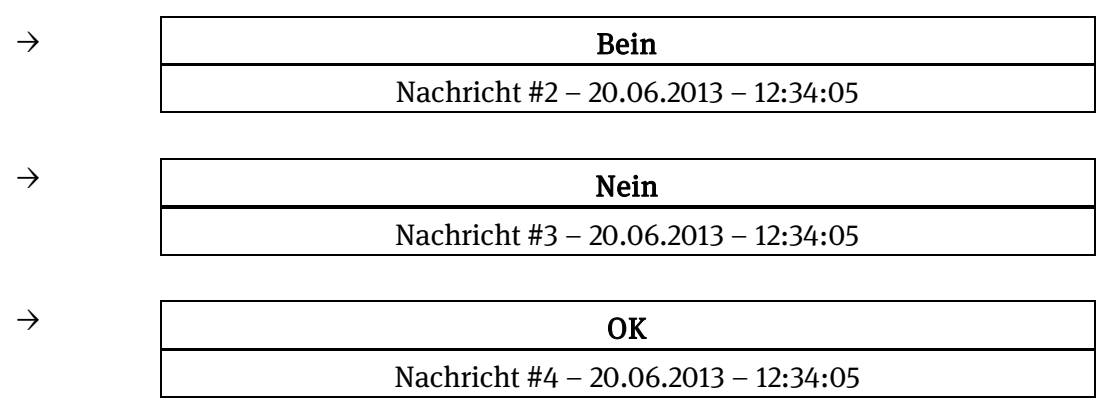

\begin{tabular}{|c|}
\hline Bein :P \\
\hline Nachricht \#5-20.06.2013-12:34:05 \\
\hline
\end{tabular}

\begin{tabular}{|c|}
\hline Oder Rot?? \\
\hline Nachricht \#6-20.06.2013-12:34:05 \\
\hline
\end{tabular}

In dieser Verabredungssequenz schlägt Schreiberin $A$ zuerst vor, sich in einer der zwei Cafeterien, der gelben Cafeteria, am Campus Essen zu treffen. Dieser Treffpunkt wird jedoch von Schreiberin $B$ abgelehnt, wobei sie ihre Ablehnung erst in \#3 nach einer selbstinitiierten Selbstkorrektur von „Bein“ (\#2) zu „Nein“ (\#3) eindeutig zum Ausdruck bringen kann. Allerdings erfolgt danach eine pragmatische Reparatur, indem die Ablehnung zurückgezogen wird und das Café als Treffpunkt in \#4 akzeptiert wird. Es kann allerdings nicht geklärt werden, aus welchem Grund die gelbe Cafeteria letztendlich doch als Treffpunkt akzeptiert wurde. Zudem kann auch keine Aussage über den zeitlichen Abstand zwischen einzelnen Nachrichten getroffen werden, da die tatsächliche Zeit, an der die jeweilige Nachricht verschickt wurde, bei der Einstellung dieses Dialogs nicht miterfasst wurde.

\section{(ii) Nonverbale Reparaturinitiierungen}

In Bezug auf das Auftreten von nicht-alphabetischen graphischen Zeichen als Reparaturmarker zeichnen sich die deutschen sowie die russischen analysierten Kurznachrichten durch den Gebrauch von Asterisken aus. Während Asteriske in den beiden Subkorpora vorkommen (jeweils 21 und sechs Fälle im deutschen und russischen Subkorpus), finden sich in den russischen Daten zwei Reparaturen, in denen Schrägstriche eine reparative Funktion übernehmen.

\section{Asteriske}

Sowohl im russischen als auch im deutschen Subkorpus werden Asteriske bei selbstinitiierten Selbstkorrekturen eingesetzt, die Problemquellen bearbeiten, 
die durch einen Vertipper oder einen Eingriff des Autokorrekturprogramms entstanden sind. Im bisherigen Verlauf der Arbeit wurden bereits zwei Belege (90) und (92) - für das Deutsche vorgestellt, in denen das Reparans jeweils mit einem vorangestellten Asterisk markiert wurde (vgl. auch (122a, b, d)). Die vorangestellte Position in Bezug auf das Reparans nehmen Asteriske im deutschen Subkorpus in 15 von 21 Fällen ein, während sechs Reparaturen nachgestellte Asteriske aufweisen. Die Belege aus dem russischen Teilkorpus zeichnen sich dagegen zwar ausschließlich durch nachgestellte Asteriske aus (vgl. (103), (105) und (120)), dies bedeutet aber nicht, dass in russischer Kurznachrichtenkommunikation die vorangestellte Position nicht vertreten ist (vgl. (102); vgl. auch Belege (56) und (77) aus der Instant-Messaging-Kommunikation):

(102) Auszug aus den für die russische Version der MoCoDa 2 erhobenen Daten:

\begin{tabular}{|c|c|c|c|}
\hline \multicolumn{3}{|c|}{$\begin{array}{l}\text { А ты, кстати, во сколько } \\
\text { прилетаешь? }\end{array}$} & \multirow[t]{2}{*}{$15: 08 \mathrm{~W}$} \\
\hline \multicolumn{3}{|r|}{6 ФЕВРАЛЯ 2018 Г. } & \\
\hline Где в 6 & $6 \quad 02: 18$ & & \\
\hline *Где тс & 02:18 & & \\
\hline
\end{tabular}

1 B: Und übrigens, um wie viel Uhr kommst du an?

6. Februar 2018

2 A: Wo um 6

3 A: *Etwa

Bei der Beantwortung der Frage nach ihrer Ankunft gibt Schreiberin $A$ in \#2 das Lokaladverb „Где“ (,wо`) anstatt des Adverbs где-то (,(a) irgendwо; (b) etwa‘) ein, bei dem das Suffix -To dem deutschen Morphem irgend- entspricht. In der Folgenachricht wird das intendierte - obgleich wegen des fehlenden Bindestrichs orthographisch nicht korrekte - Adverb „Где то“ (im gegebenen Kontext: ,etwa') geliefert.

Die mit Asterisken gekennzeichneten Reparantia kommen in den in der vorliegenden Arbeit analysierten Daten ausschließlich bei Substitutionen vor (vgl. aber Mostovaia 2018, die zeigt, dass Asteriske in der deutschen WhatsApp-Kommunikation auch nachträglich einzufügende Elemente markieren können; vgl. (63)). Dabei werden dem Rezipienten mit Ausnahme von drei Reparaturen aus dem russischen Subkorpus - darunter auch (103) und (105) - keine Anhaltspunkte bei der Lokalisierung des zu ersetzenden Reparandums zur Verfügung gestellt. Das Reparandum muss somit entweder durch seine semantische oder syntaktische Übereinstimmung (vgl. Pfeiffer 2015: 262-263) bzw. durch seine partielle graphische Ähnlichkeit mit dem Reparans vom Rezipienten selbst ermittelt werden. Lediglich in zwei russischen Belegen kommt jeweils ein syntaktischer Anker vor, und zwar einmal der postpositionierte (vgl. \#2812 in der $M o C o D a)$ und einmal eine Kombination aus dem präpositionierten und postpo- 
sitionierten syntaktischen Anker (vgl. (103)). Des Weiteren wird in (105), in dem der Asterisk zusammen mit einem Schrägstrich verwendet wird, die Lokalisierung des Reparandums durch seine Wiederaufnahme gewährleistet (vgl. den Abschnitt zu Schrägstrichen). Hier soll nun anhand eines Auszugs aus dem russischen Korpus das gleichzeitige Auftreten des präpositionierten und postpositionierten syntaktischen Ankers veranschaulicht werden:

(103) Auszug aus dem SMS-Dialog \#2494:

Schreiberin $A$

Schreiberin $B$

\begin{tabular}{|c|}
\hline Я звонила в аркаим. Сегодня аэробики не будет \\
\hline $\begin{array}{c}\text { Ich habe bei „Arkaim“ angerufen. Heute findet der Aerobic-Kurs nicht } \\
\text { statt. }\end{array}$ \\
\hline Nachricht \#1 -17.07.2013 - 12:52:00 \\
\hline
\end{tabular}

\begin{tabular}{|c|}
\hline Ага,знаю \\
\hline Ja, weiß ich \\
\hline Nachricht \#2 $-17.07 .2013-12: 54: 00$ \\
\hline
\end{tabular}

$\rightarrow$

Прикинь, у надо до 6 часов не будет света, дикость такая..

Hast du schon gehört, dass es bei muss bis 18 Uhr keinen Strom geben wird, was für eine Frechheit...

Nachricht \#3 - 17.07.2013 - 12:56:00

$\rightarrow$

\begin{tabular}{|c|}
\hline У нас до \\
\hline Bei uns bis $^{\star}$ \\
\hline Nachricht \#4 $-17.07 .2013-12: 56: 15$ \\
\hline
\end{tabular}

Тебе тоже отключили электричество?

Wurde bei dir auch der Strom abgeschaltet?

Nachricht \#5 - 17.07.2013 - 12:56:55

\begin{tabular}{|c|}
\hline У нас тоже... \\
\hline Bei uns auch... \\
\hline Nachricht \#6 $-17.07 .2013-12: 57: 05$ \\
\hline
\end{tabular}

In Auszug (103) teilt Schreiberin $A$ ihrer Freundin mit, dass der Aerobic-Kurs, den sie beide besuchen, ausfällt. In der sich daraus entwickelnden Kommunikation beschwert sich Schreiberin $B$ bei Schreiberin $A$ über den Stromausfall, der 
voraussichtlich erst am Abend behoben wird, und fragt Schreiberin $A$, ob sie ebenfalls davon betroffen wurde. Dabei produziert sie in dem Abschnitt „у надо до 6 часов не будет света“ das Wort „надо“ (,man muss, nötig sein`) anstatt von нас (,uns'). In der darauffolgenden Nachricht führt sie eine selbstinitiierte Selbstkorrektur durch, indem sie zum Anfang der Präpositionalphrase, die die Problemquelle „надо“ ursprünglich enthält, retrahiert, die Präposition „y“ (,bei“) als präpositionierten syntaktischen Anker realisiert und anschließend das Reparans „нас“ (,uns`) sowie die Präposition „до“ (,bis`) als postpositionierten syntaktischen Anker liefert. Die Tatsache, dass lediglich die Präposition „до“ (,bis‘) und nicht die ganze Präpositionalphrase „до 6 часов“ (,bis 18 Uhr`) als postpositionierter syntaktischer Anker fungiert, lässt sich wahrscheinlich damit erklären, dass die Wiederholung eines Wortes nach dem Reparans „нас“ (,uns`) seine Anbindung an die ursprüngliche syntaktische Struktur (vgl. Pfeiffer 2015: 262-264) ausreichend anzeigt. Daher kann auf die weitere Fortführung der Präpositionalphrase aus der Nachricht mit der Problemquelle verzichtet werden, da das Eintippen mehrerer Wörter gewisse Zeit in Anspruch nimmt und somit die Kommunikation verlangsamt.

\section{Schrägstriche}

In zwei selbstinitiierten Selbstreparaturen aus dem russischen Subkorpus erfolgt die Substitution jeweils im Format Reparans/Reparandum (vgl. (104) und (105)), wobei die Wiederaufnahme der Problemquelle für ihre eindeutige Lokalisierung sorgt, während der Schrägstrich zur Herstellung eines Bezugs zwischen dem wiederholten Reparandum und dem gelieferten Reparans beiträgt (vgl. auch Gleichheitszeichen sowie Bindestriche in der gleichen Funktion in 3.4.2.1). Im Gegensatz zu dem bei Cherny (1999: 93) für die Chat-Kommunikation beschriebenen Reparaturen, die ebenso Schrägstriche enthalten und nach dem Muster s/Reparandum/Reparans aufgebaut sind, fällt bei den zwei Beispielen aus der russischen WhatsApp-Kommunikation das $s$ weg, das in den Daten von Cherny (1999) die Substitution als Reparaturoperation kennzeichnet. Zudem weisen die Reparaturen im Russischen eine andere Reihenfolge des Reparandums und des Reparans auf. Das Reparans, das als Träger neuer Information eine höhere Relevanz aufweist, wird vor das Reparandum gestellt:

(104) Auszug aus dem WhatsApp-Dialog \#4355:

Schreiberin $A$

Schreiberin $B$

\begin{tabular}{|c|}
\hline А что такое медиация? \\
\hline Und was ist Mediation? \\
\hline Nachricht \#4 - 30.01.18 - 20:15 \\
\hline
\end{tabular}




\begin{tabular}{|c|}
\hline Я не перенесу такой позор, когда покажу тебе свои писанины $\Theta$ \\
\hline Ich schäme mich etwas, dir mein Geschreibsel zu zeigen \\
\hline Nachricht \#5 - 30.01.18 - 20:16 \\
\hline
\end{tabular}

\begin{tabular}{|c|}
\hline $\begin{array}{c}\text { Это колледжа ты помогаешь людям урегулировать конфликт } \\
\text { любого плана, опираясь больше на определённые техники и } \\
\text { психологию. Ты не судья, а как бы третье лицо, которое помогает } \\
\text { разрулить конфликт }\end{array}$ \\
\hline $\begin{array}{c}\text { Das ist, Berufsschule du Menschen hilfst, einen Streit jeglicher Art } \\
\text { zu lösen, indem du vor allem auf bestimmte Methoden und die } \\
\text { Psychologie zurückgreifst. Du trittst dabei nicht als Richter, sondern } \\
\text { als Dritter, der hilft, den Streit zu lösen, auf }\end{array}$ \\
\hline Nachricht \#6 - 30.01.18 - 20:17 \\
\hline
\end{tabular}

\begin{tabular}{c|c|}
\hline Ах, я тебя умоляюе \\
\hline Ach komm, ich bitte dich! \\
\hline Nachricht \#7 - 30.01.18 - 20:17 \\
\hline Kогда/колледжа \\
\hline Wenn/Berufsschule \\
\hline Nachricht \#8 - 30.01.18 - 20:17 \\
\hline
\end{tabular}

In WhatsApp-Dialog (104) zwischen zwei Freundinnen berichtet Schreiberin $A$ in einer hier nicht abgebildeten Nachricht darüber, welche Leistungen sie während ihres Auslandssemesters an einer deutschen Universität noch erbringen soll. Dabei erwähnt sie das Thema Mediation, welches in einem ihrer Kurse behandelt wurde, was durch $B$ mit einer Frage, die eigentlich als eine Fremdinitiierung zu werten ist (vgl. Kap. $6.2 \mathrm{zu}$ fremdinitiierten Selbstreparaturen), aufgegriffen wird. Der problematische Begriff wird von $A$ in \#6 erläutert. Dabei unterläuft ihr ein anscheinend der Autokorrektur verschuldeter Fehler, sodass das Wort „колледжа“ (,College-GEN` bzw. ,Berufsschule-GEN`) anstelle von когда (,wann/wenn') erscheint. In \#8 unternimmt $A$ eine Korrektur, indem sie das Reparans „Когда“ (,wenn') gefolgt von einem Schrägstrich sowie dem Reparandum „колледжа“ (,Berufsschule-GEN`) verschickt.

Das nächste Beispiel, in dem das Reparans und das Reparandum ebenso durch einen Schrägstrich voneinander abgetrennt sind, zeigt, dass einzelne nicht-alphabetische Reparaturmarker miteinander kombiniert werden können. In dem Fall liegt in (105) die Kombination aus einem Schrägstrich und einem nachgestellten Asterisk vor: 
(105) Auszug aus dem WhatsApp-Dialog \#4354:

Schreiberin $A$

Schreiberin $B$

Решила опробовать новое корейское место, но мне не очень понравилось

Ich wollte mal ein anderes koreanisches Lokal ausprobieren, aber es hat mir nicht so sehr gefallen

Nachricht \#16 - 15.02.2018 - 15:42

\begin{tabular}{|c|}
\hline Невкусно? \\
\hline Hat es dir nicht geschmeckt? \\
\hline Nachricht \#17 - 15.02.2018 - 15:42 \\
\hline
\end{tabular}

\begin{tabular}{|c|}
\hline Пресновато как-то \\
\hline Es war irgendwie fade \\
\hline Nachricht \#18 - 15.02.2018 - 15:42 \\
\hline
\end{tabular}

\begin{tabular}{|c|}
\hline Или атмосфера не располагает? \\
\hline Oder hat dir die Atmosphäre nicht zugesagt? \\
\hline Nachricht \#19 - 15.02.2018 - 15:42 \\
\hline
\end{tabular}

\begin{tabular}{|c|c|}
\hline & $\begin{array}{c}\text { Нет, я все умяла, особенно с голода и атмосфера кстати милая, а } \\
\text { вот кожа могла бы быть лучше. Я в другом корейском ресторане } \\
\text { было и то же блюдо там было намного вкуснее. }\end{array}$ \\
\hline & $\begin{array}{l}\text { Doch, ich habe alles leer geputzt, weil ich so hungrig war, und es ist } \\
\text { eigentlich auch ganz gemütlich da, aber die Haut hätte besser sein } \\
\text { können. Ich war mal in einem anderen koreanischen Restaurant, und } \\
\text { dasselbe Gericht war um einiges leckerer. }\end{array}$ \\
\hline & Nachricht \#20 - 15.02.2018 - 15:43 \\
\hline \multirow[t]{4}{*}{$\rightarrow$} & Еда/кожа * \\
\hline & Essen/Haut * \\
\hline & Nachricht \#21 - 15.02.2018 - 15:44 \\
\hline & (eine Nachricht ausgelassen) \\
\hline & Людоедка $\Leftrightarrow \Leftrightarrow$ \\
\hline & Du Kannibalin $\Leftrightarrow \Leftrightarrow$ \\
\hline & Nachricht \#23 - 15.02.2018 - 15:44 \\
\hline
\end{tabular}


In (105) teilt Schreiberin $B$ ihre Eindrücke über ein koreanisches Restaurant, in dem sie zum ersten Mal war, mit Schreiberin $A$ und gibt in \#20 u.a. an, dass die Haut besser sein könnte (,,а вот кожа могла бы быть лучше“). In der nächsten Nachricht führt $B$ eine Substitution durch, indem sie „кожа“ (,Haut') durch „Еда“ (,Essen`) ersetzt, wobei die vorliegende Reparaturoperation redundant gekennzeichnet wird, und zwar einmal durch den nachgestellten Asterisk und einmal durch den Schrägstrich sowie das Vorhandesein des wiederholten Reparandums. Da im gesamten Korpus jedoch lediglich zwei Fälle der Verwendung von Schrägstrichen auftreten - darunter ein Fall (105), in dem der Schrägstrich mit einem Asterisk kombiniert wird - und beide von der gleichen Schreiberin stammen, ist es nicht auszuschließen, dass es sich dabei um eine idiosynkratische Konvention handelt, eine Substitution $\mathrm{zu}$ markieren. $\mathrm{Ob}$ sich Schrägstriche als Reparaturmarker auch bei anderen Schreibern finden, muss in zukünftigen Studien an einem größeren Korpus untersucht werden.

\section{(iii) Lexikalische Reparaturinitiierungen}

Die Gruppe lexikalischer Reparaturinitiierungen umfasst im Gegensatz zu phrasalen und satzwertigen Reparaturinitiierungen (vgl. (111)-(113)) diejenigen Reparaturmarker, die nicht phrasenfähig sind (v.a. Partikeln und Konjunktionen; vgl. Imo 2016: 137). Zudem werden dieser Kategorie auch einzelne phrasenfähige Reparaturmarker zugeordnet, die allerdings - anders als phrasale Reparaturmarker - nicht erweiterte, nur aus dem Kopf bestehende Phrasen bilden und - anders als satzwertige Reparaturmarker - nicht als Ellipse interpretiert werden können.

\section{Partikeln}

Partikeln als Reparaturmarker treten in den analysierten Kurznachrichten überwiegend bei lexiko-semantischen sowie pragmatischen Reparaturen (meist Elaborierungen) auf. Dabei zeichnet sich das russische Subkorpus durch den Gebrauch der Partikel $H y$ (,na ja, nun, halt, äh') aus, die in zwei Reparaturinitiierungen alleine und in zwei weiteren in Kombination mit einer Konjunktion vorkommt. In den deutschen Daten dagegen sind unterschiedliche Partikeln vertreten, und zwar: ach und ne(e) (jeweils fünf Tokens; vgl. (109) und (118)), nicht (zwei Tokens; vgl. (97)), doch (zwei Tokens) sowie ah, äh, ehm (vgl. (114)), na, nein, oh (vgl. 122c) und ups (vgl. (121)) (jeweils ein Token). Der Diskursmarker also (vgl. Dittmar 2002), der in 17 Fällen als Reparaturmarker gebraucht wird, wird ebenfalls der Gruppe der Partikeln zugeordnet. Die Entschuldigungsfloskel sorry, die drei Mal in selbstinitiierten Selbstreparaturen im deutschen Subkorpus auftritt, wird in Anlehnung an die Duden-Grammatik (2016: 606), die 
Entschuldigung als Gesprächspartikel einstuft, als Partikel angesehen (zu sorry als Reparaturmarker vgl. Fußnote 101). Laut Muryasov (2018: 939) ließe sich die Entschuldigungsfloskel прости (,sorry'), die in einem der Belege aus den russischen Daten auftritt (vgl. (99)), als eine deverbative Interjektion auffassen. Da Interjektionen im Rahmen dieser Arbeit als eine der Partikelklassen angesehen werden (vgl. Duden 2016: 609-610), wird прости (,sorry') hier ebenso als eine Partikel behandelt.

Für $н y$, das ins Deutsche schwer übersetzbar ist und je nach dem Kontext mit na ja, nun, halt, äh usw. wiedergegeben werden kann, listen Tsesarskaya/Shestopalova (2017: 70) drei Verwendungskontexte auf (vgl. Kap. 2.2.1.1). Erstens kann $н y$ bei prospektiven Reparaturen verwendet werden, also bei Wortsuchen, sodass $H y$ dabei mit „Ich kann das passende Wort nicht finden.“ umschrieben werden kann (vgl. Tsesarskaya/Shestopalova 2017: 70). Des Weiteren tritt $н y$ auch bei retrospektiven Reparaturen auf, also bei solchen, die sich auf eine bereits realisierte Konstituente beziehen. Dabei unterscheiden Tsesarskaya/Shestopalova (2017: 70) Fälle, bei denen $H y$ entweder eine Substitution oder eine Insertion einleitet. Die Verwendung von $н y$ im Rahmen einer ,inszenierten“ prospektiven Reparatur wird im Kapitel 6.1.2 zu selbstinitiierten Selbstreparaturen im same turn anhand des Beispiels (134) veranschaulicht. Eine retrospektive mit positionalphrase zum Zweck der Bedeutungspräzisierung des gewählten sprachlichen Ausdrucks erfolgt, wurde bereits in (95) vorgestellt. Mit (106) wird nun der Gebrauch von $\boldsymbol{y} y$ im Rahmen einer retrospektiven Substitution veranschaulicht:

(106) Auszug aus dem SMS-Dialog \#3071:

Schreiberin $A$

Schreiberin $B$

\begin{tabular}{|c|}
\hline мне приснилось,что у меня есть черный кот и его зовут бэтмен.. \\
\hline ich habe von einem schwarzen Kater geträumt, der Batman heißt.. \\
\hline Nachricht \#1 - 17.12.2013 - 20:00 \\
\hline
\end{tabular}

\begin{tabular}{|c|}
\hline ШИКАРНО \\
\hline VOLL SCHICK \\
\hline Nachricht \#2 $-17.12 .2013-20: 01$ \\
\hline
\end{tabular}

$\rightarrow \mid$\begin{tabular}{|c|}
\hline и у него было пятно белое на шее.. \\
\hline und er hatte einen weißen Fleck an seinem Hals.. \\
\hline Nachricht \#3 - 17.12.2013 - 20:01 \\
\hline
\end{tabular}




\begin{tabular}{|c|}
\hline какой..(( \\
\hline so einer..(( \\
\hline Nachricht \#4 - 17.12.2013 - 20:02 \\
\hline
\end{tabular}

$\rightarrow$\begin{tabular}{c|}
\hline ну не на шее, а там где значек у бэтмена \\
\hline $\begin{array}{c}\text { naja nicht am Hals, sondern da, wo sich das Logo auf dem } \\
\text { Batmankostüm befindet }\end{array}$ \\
\hline Nachricht \#5 - 17.12.2013-20:04 \\
\hline
\end{tabular}

\begin{tabular}{|c|}
\hline$\wedge \wedge$ \\
\hline$\wedge \wedge$ \\
\hline Nachricht \#6 $-17.12 .2013-20: 06$ \\
\hline
\end{tabular}

\begin{tabular}{|c|}
\hline тоже овальное такое \\
\hline auch so länglichrund \\
\hline Nachricht \#7 - 17.12.2013 - 20:07 \\
\hline
\end{tabular}

\begin{tabular}{|c|}
\hline интересно, что об этом думает фрейд.. \\
\hline interessant, was würde Freud dazu sagen.. \\
\hline Nachricht \#8 - 17.12.2013 - 20:08 \\
\hline
\end{tabular}

$\mathrm{Zu}$ Beginn des in (106) abgebildeten Auszugs berichtet Schreiberin $A$ ihrer Freundin davon, dass sie von einem schwarzen Kater namens Batman geträumt hat. In \#3 beschreibt $A$ diesen Kater und merkt an, dass er einen weißen Fleck an seinem Hals hatte. In \#5 nimmt $A$ aber ihre Worte darüber zurück und teilt mit, dass der Fleck genau da war, wo sich das Logo auf dem Kostüm von Batman befindet, sodass man eher von einem weißen Brustfleck ausgehen kann. In dem Fall wird also das Reparandum „на шее“ (,an seinem Hals`) wiederholt, verneint und anschließend durch das Reparans „там где значек у бэтмена“ (,da, wo sich das Logo auf dem Batmankostüm befindet‘) substituiert (zu Substitutionen im Format nicht Reparandum - Reparans vgl. Pfeiffer 2015: 303-304).

Im Gegensatz zu von Partikeln in der untersuchten russischen Kurznachrichtenkommunikation festgestellt werden, die von Tsesarskaya/Shestopalova (2017) für die russische gesprochene Sprache belegt wurden. Dazu gehören Reparaturmarker wie даже (,sogar'), ой(,ups'), тьфу (,pfui') sowie блин (,Mist'). Die Frage, inwiefern diese Partikeln von Schreibern in der Kurznachrichtenkommunikation zu Reparaturzwecken eingesetzt werden, stellt zurzeit noch ein Forschungsdesiderat dar. Beispiele aus der MoCoDa 2 zeigen allerdings, dass zumindest die Partikel ой 
(,ups`) sowie eine weitere aus dem Englischen entlehnte Partikel упс ([ups] ,ups`) in der russischen WhatsApp-Kommunikation eine bevorstehende selbstinitiierte Selbstreparatur ankündigen können. Die Partikel оц̆ (,ups`) geht der in (107) vorliegenden selbstinitiierten Selbstreparatur voran, die auf das Zombie-Emoji $=$ (\#2) gerichtet ist.

(107) Auszug aus den für die russische Version der MoCoDa 2 erhobenen Daten:

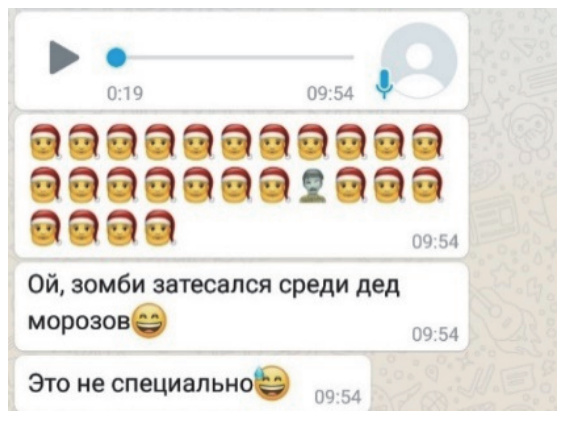

1 A: (Sprachnachricht)

\section{A:

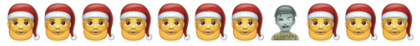 คำ으}

3 A: Ups, zwischen die Väterchen Frost hat sich ein Zombie eingeschlichen $\theta$

4. A: War aus Versehen

Der in \#1 verschickten Sprachnachricht, die eine Aufnahme eines Weihnachtslieds darstellt, folgen mehrere aneinandergereihte ,Santa'-Emojis, denen in dem Fall eine ludische Funktion (vgl. Pappert 2017: 202-204) zugeschrieben werden kann. In der zweiten Zeile erscheint aber zwischen ,Santa'-Emojis ein ,Zombie‘Emoji, dessen Vorkommen von $A$ in der nächsten Nachricht als ein Fehler thematisiert wird. Dabei deutet sie mit der Partikel ой(,ups') an, dass ihr ein Missgeschick passiert ist, liefert danach eine explizite Erklärung als Reparaturdurchführung und merkt anschließend noch an, dass das keine Absicht war.

In den deutschen Daten finden sich insgesamt 37 Partikeln, die allerdings miteinander sowie mit anderen Initiierungsressourcen kombiniert werden und sich daher auf 32 Reparaturinitiierungen verteilen. Fast die Hälfte aller Partikeln macht der Diskursmarker also aus, der mit 17 Tokens nach Asterisken als nichtalphabetischen graphischen Reparaturmarkern die zweitgrößte Gruppe von expliziten Reparaturmarkern darstellt. Laut Pfeiffer (2017b: 277-278, 280) verfügt also über ein breites Funktionsspektrum, da dies sowohl bei retrospektiven als auch bei prospektiven Reparaturen eingesetzt werden kann. Bei retrospektiven Reparaturen handelt es sich dabei meistens um Elaborierungen, in deren Rahmen eine Substitution durchgeführt wird (vgl. Pfeiffer 2015: 175-176, 2017: 280). Unter den analysierten selbstinitiierten Selbstreparaturen mit also können die meisten ebenso den Elaborierungen zugerechnet werden, welche v.a. die Referenz sowie die pragmatische Dimension einer Nachricht betreffen. Ein Bei- 
spiel, in dem eine mit also eingeleitete Selbstreparatur auf ein pragmatisches Reparandum gerichtet ist, findet sich in (108):

(108) SMS-Dialog \#3935:

Schreiber $A$

Schreiberin $B$

Kathi von wem is der sommerhit den du mir im Auto gezeigt hat? Kannst

du das raus find $3 n ? ?$

Nachricht \#1 - 28.12.2015 - 22:23

Feder :)

Nachricht \#2 - 28.12.2015 - 22:30

Danke!! Und wie heißt der Song? :)

Nachricht \#3 - 28.12.2015 - 22:31

Das weiß ich nicht grad. Aber findste bestimmt schnell auf YouTube

Nachricht \#4 - 28.12.2015 - 22:35

$\rightarrow$

\begin{tabular}{|c|}
\hline Goodbye \\
\hline Nachricht \#5 - 28.12.2015-22:35 \\
\hline
\end{tabular}

$\rightarrow$

\begin{tabular}{|c|}
\hline Also so heißt der Song ;) Gute Nacht \\
\hline Nachricht \#6 - 28.12.2015 - 22:40 \\
\hline
\end{tabular}

\begin{tabular}{|c|}
\hline Dankeeee $:^{-\star}$ good night \\
\hline Nachricht \#7 $-28.12 .2015-22: 52$ \\
\hline
\end{tabular}

In \#3 fragt Schreiber $A$ seine Schwester nach dem Titel des Songs, den sie ihm vorher im Auto vorgespielt hat. Schreiberin $B$ kann aber in jenem Moment diese Frage nicht beantworten und rät Schreiber $A$, den Titel bei YouTube selbst zu recherchieren. Wenige Sekunden später verschickt $B$ jedoch Nachricht \#5 mit dem einzigen Wort „Goodbye“, welches man in dem Kontext u.U. als eine Verabschiedungsfloskel interpretieren könnte, die allerdings durch ihren unmittelbaren Anschluss an das - zwar begründete - Ausbleiben der Antwort in \#4 einen für $A$ gesichtsbedrohenden Charakter erhält. Die in \#6 durchgeführte Elaborierung stellt klar, dass Nachricht \#5 nicht als eingeleitete Abschiedssequenz, sondern als die Antwort auf $A$ 's Frage nach dem Titel des Songs (\#3) zu verstehen ist. Also als Reparaturmarker trägt dabei zur Herstellung der ,erläuternden Relation“ (vgl. Pfeiffer 2017b: 280) zwischen dem Reparandum 
„Goodbye“ (\#5) und seiner nachträglichen Interpretation in \#6 bei. Somit wird der interaktionale Bezug von Nachricht \#5 verdeutlicht, sodass diese als Antwort kein gesichtsbedrohendes Potenzial mehr für $A$ hat.

Die zwei weiteren Partikeln - der Erkenntnisprozessmarker ach sowie die Partikel ne(e) - sind im deutschen Subkorpus jeweils mit fünf Tokens vertreten, wobei sie drei Mal in Kombination miteinander in einer Reparaturinitiierung gebraucht werden. ${ }^{104}$ Der Einsatz der beiden Partikeln ist auch bereits für Reparaturen in der gesprochenen Sprache belegt (für ach vgl. Willkop 1988: 210-219; Egbert 2009: 59; Pfeiffer 2015: 293, 2017b: 275; für nee vgl. Willkop 1988: 166169; Pfeiffer 2015: 299-300; Imo 2017: 64-67). Dabei weist Pfeiffer (2017b: 275) darauf hin, dass ach, das als Reparaturmarker in seinen Daten eine semantische Korrektur markiert, eine stark ausgeprägte emotionale Komponente hat. Daher kann ach von den Sprechern eingesetzt werden, um ,ihre Verwunderung oder gar Verärgerung darüber ausdrücken, dass ihnen bei der Hervorbringung ihrer Äußerung versehentlich ein reparaturbedürftiger inhaltlicher Fehler unterlaufen ist“ (Pfeiffer 2017b: 275). Außerdem nimmt Pfeiffer (2017b: 275) an, dass ach in solchen Kontexten verwendet wird, in denen Schreiber u.a. auf „die offensichtliche Korrekturbedürftigkeit“ der zurückliegenden Reparanda verweisen. Die Partikel ne(e) wird laut Pfeiffer (2015, 2017b: 273-274) ebenfalls in Reparaturinitiierungen bei semantischen Korrekturen verwendet, während Imo (2017: 65-66) nee eine weitere reparative Funktion zuschreibt, nämlich die Kennzeichnung des Wechsels der Interaktionsmodalität. In den in der vorliegenden Arbeit analysierten Reparaturen wird das alleinstehende ach sowie ach in Kombination mit $n e(e)$ bei lexiko-semantischen und pragmatischen Korrekturen verwendet (vgl. (109)), wohingegen das alleinstehende ne(e) bzw. ne(e) in Verbindung mit also einen Modalitätswechsel markiert (vgl. \#2386 in der MoCoDa):

(109) Auszug aus dem Dialog \#2348:

Schreiberin $A$

Schreiber $B$

\begin{tabular}{|c|}
\hline$<3$ Schatz, musst du nicht zur Uni? \\
\hline Nachricht \#1 - 30.10.2013 - 08:40:00 \\
\hline
\end{tabular}

104 Egbert (2009: 63-64) führt zwar auch ein Beispiel für ach nee im Kontext einer selbstinitiierten Selbstreparatur an (,warum nich- ach nee du wars ja tee ha wee ne?“, Hervorhebung im Original), betrachtet diese Partikelkombination allerdings nicht als Reparaturinitiierung, sondern als eine Art ,gefüllte“ Verzögerung, die zwischen der Reparaturinitiierung in Form eines Abbruchs und der Reparaturoperation platziert ist. 
Guten Morgen Schatz, bin gerade wach. Klar muss gleich los. Heute bis $18 \mathrm{Uhr}$, dann arbeiten.

Nachricht \#2 - 30.10.2013 - 08:50:00

\begin{tabular}{|c|c|}
\hline \multirow[t]{2}{*}{$\rightarrow$} & $\begin{array}{l}\text { Haben Training danach gucken wir son Motivationsfilm im Kino. } \\
\text { Kommst du Samstag schnell zu mir?<3<3 Montag musst du vielleicht } \\
\text { was entgegen nehmen. Stühle kommen am 13.11. }\end{array}$ \\
\hline & Nachricht \#3-30.10.2013-09:05:00 \\
\hline \multirow[t]{2}{*}{$\rightarrow$} & Ach nee, Mittwoch ist das ne?! Notier schnell :-) \\
\hline & Nachricht \#4 - 30.10.2013 - 09:28:00 \\
\hline
\end{tabular}

Ich bin Montag bei dir. Ich hab in der nächsten Woche komplett frei, bis auf ein paar Ausnahmen. Ist Prüfungswoche bei uns. Und dann bleib ich bis Dienstag Morgen auf jeden Fall in Osna.

Nachricht \#5 - 30.10.2013 - 09:29:00

Im Laufe eines Dialogs zwischen einem Pärchen informiert Schreiber $B$ seine Freundin in \#3 u.a. darüber, dass sie für ihn am Montag möglicherweise etwas entgegennehmen muss. In der gleichen Nachricht teilt er ihr außerdem mit, dass am 13. November Stühle geliefert werden. Daraus lässt sich jedoch schließen, dass es sich hier um zwei voneinander unabhängige Lieferungen geht, da der 13. November 2013 ein Mittwoch ist. Die in \#4 erfolgte selbstinitiierte Selbstreparatur bearbeitet aber eine der Zeitreferenzen, und zwar den Wochentag („Ach nee, Mittwoch ist das ne?!“ in \#4), sodass klar wird, dass es in \#3 doch um dieselbe Lieferung ging.

\section{Konjunktionen}

Konjunktionen als lexikalische Reparaturinitiierungen sind jeweils mit zwei Tokens im deutschen und vier im russischen Teilkorpus belegt: Für die deutschen Daten sind das bzw. (vgl. \#2456 in der MoCoDa) und oder (vgl. \#2386 in der $M o C o D a$ ), wobei das Letzte in Kombination mit einer satzwertigen Reparaturinitiierung auftritt. In den russischen Daten liegen einerseits zwei Reparaturinitiierungen mit der Konjunktion или (,oder') und andererseits zwei mit der Konjunktion то есть (,das heißt') vor, die laut Tsesarskaya/Shestopalova (2017: 66-68) in der gesprochenen Sprache sowohl bei Elaborierungen als auch bei Korrekturen eingesetzt werden kann. Eine Elaborierung mit то есть (,das heißt') wurde bereits im Kapitel zu lexiko-semantischen Reparanda diskutiert 
(vgl. (95)), hier soll nun eine mit dem gleichen Reparaturmarker eingeleitete pragmatische Korrektur vorgestellt werden:

(110) Auszug aus dem SMS-Dialog \#3052:

Schreiber $A$

Schreiberin $B$

\begin{tabular}{|c|}
\hline Привет!:)Встретимся? \\
\hline Hallo!:) Lass uns mal treffen? \\
\hline Nachricht \#1 - 08.10.2013 - 21:50:00 \\
\hline
\end{tabular}

\begin{tabular}{|c|}
\hline зачем? \\
\hline wozu? \\
\hline Nachricht \#2 $-08.10 .2013-21: 51: 00$ \\
\hline
\end{tabular}

\begin{tabular}{|c|}
\hline Это был вопрос:-) \\
\hline Das war eine Frage:-) \\
\hline Nachricht \#3 - 08.10.2013 - 21:52:00 \\
\hline
\end{tabular}

\begin{tabular}{|c|}
\hline скорее да чем нет \\
\hline eher ja als nein \\
\hline Nachricht \#4 - 08.10.2013 - 21:53:00 \\
\hline т.е. скорее нет чем да \\
\hline d.h. umgekehrt, eher nein als ja \\
\hline Nachricht \#5 - 08.10.2013 - 21:53:30 \\
\hline А чтож так :( \\
\hline Wieso denn :( \\
\hline Nachricht \#6 - 08.10.2013 - 21:55:00 \\
\hline времени совсем нет \\
\hline ich habe überhaupt keine Zeit \\
\hline Nachricht \#7 - 08.10.2013 - 21:55:30 \\
\hline
\end{tabular}

In dem oben abgebildeten Ausschnitt unterbreitet Schreiber $A$ ein Verabredungsangebot, welches von seiner Bekannten, Schreiberin $B$, zuerst in Bezug auf den Zweck des Treffens hinterfragt und in \#4 anschließend doch angenommen wird. Nachricht \#4, in der $B A$ 's Angebot angenommen hat, stellt sich jedoch in \#5 als Problemquelle heraus, da $B$ innerhalb von 30 Sekunden ihre 
bestätigende Antwort zurückzieht und durch eine Ablehnung ersetzt. Die dadurch erfolgte pragmatische Selbstkorrektur leitet $A$ mit der für die geschriebene russische Sprache etablierten Abkürzung für то есть (,das heißt') - nämlich „т.е.“ (,d.h.`) - ein. Anschließend kündigt sie durch das Adverb „наоборот“ (,umgekehrt') die bevorstehende Korrektur an, in deren Rahmen „да“ (,ja') und „нет“ (,nein') aus der ursprünglichen Nachricht vertauscht werden, sodass keine Zusage, sondern eine Ablehnung zustande kommt.

\section{(iv) Phrasale und satzwertige Reparaturmarker}

Die letzte Gruppe von Reparaturmarkern, die in den untersuchten Kurznachrichten vertreten ist, schließt phrasale und satzwertige Reparaturinitiierungen mit rein und umfasst 15 Belege im deutschen und fünf im russischen Subkorpus. Manche dieser Reparaturmarker weisen einen floskelhaften Charakter auf: Dazu gehören u.a. der Reparaturmarker в смысле (,im Sinne von, ich meine, soll heißen') (ein Token, vgl. (111)) und Reparaturinitiierungen mit Verben meinen (sieben Tokens) und heißen (zwei Tokens, vgl. (98)) sowie ich habe mich vertan (ein Token vgl. (113)).

In der russischen gesprochenen Sprache wird der phrasale Reparaturmarker в смысле (,im Sinne von') hauptsächlich in Elaborierungen verwendet, in denen die implizite Bedeutung eines Teils der Nachricht bzw. der gesamten Nachricht expliziert wird (vgl. Tsesarskaya/Shestopalova 2017: 66-68). In SMSDialog (111) wird diese Reparaturinitiierung eingesetzt, um die in \#2 vorliegende subtile Implikatur klarer zu machen:

(111) Auszug aus dem SMS-Dialog \#3434:

Schreiberin $A$

Schreiberin $B$

\begin{tabular}{|c|}
\hline Как насчет съездить в Бонн? Здравствуйте!:-) \\
\hline Wie wäre es damit, nach Bonn zu fahren? Guten Tag! :-) \\
\hline Nachricht \#1 - 15.04.2014 - 11:59:28 \\
\hline
\end{tabular}

$\rightarrow$

\begin{tabular}{|c|}
\hline Ja pioner! $:-$ \\
\hline Ich bin ein Pionier! $:-$ \\
\hline Nachricht \#2 $-15.04 .2014-12: 00: 09$ \\
\hline
\end{tabular}

$\rightarrow$

\begin{tabular}{|c|}
\hline V smisle vsegda gotov:-) \\
\hline Im Sinne von bin immer bereit :-) \\
\hline Nachricht \#3 - 15.04.2014 - 12:09:02 \\
\hline
\end{tabular}




\begin{tabular}{|c|}
\hline Ну, все. Едем! Девчонок можно позвать. \\
\hline Na dann fahren wir! Wir können noch die Mädels fragen, ob sie \\
mitfahren wollen. \\
\hline Nachricht \#4-15.04.2014 - 12:14:38 \\
\hline
\end{tabular}

In Dialog (111), der zwischen zwei Freundinnen verläuft, schlägt Schreiberin $A$ vor, einen Ausflug nach Bonn zu unternehmen. Darauf antwortet Schreiberin $B$, die ihre Nachricht in lateinischer Umschrift - dem sogenannten Translit (vgl. Kap. 4.1) - verfasst, mit „Ja pioner!“ (,Ich bin ein Pionier!'), was auf den ersten Blick weder als Ablehnung noch als Zustimmung gedeutet werden kann. Mit ihrer Antwort bezieht sich $B$ aber auf die im Russischen inzwischen floskelhafte Phrase $Я$ как пионер, всегда готов! (,Ich als Pionier bin für alles immer bereit!‘), die auf die Grußformel „vsegda gotov“ (,Immer bereit!`) von Pionieren, also Mitgliedern einer Jugendorganisation aus der Sowjetzeit, zurückgeht. Heutzutage wird diese Phrase v.a. in zwei Bedeutungen verwendet: Zum einen bringt man damit zum Ausdruck, dass man immer auf der Hut und somit auf alles vorbereitet ist. Zum anderen - und diese Bedeutung trifft für (111) zu signalisiert man mit dieser Phrase, dass man für alles bereit bzw. für alles zu haben ist. Das Wort „pioner“ (,Pionier`) gibt jedoch aus $B$ 's Sicht nicht ausreichend Anhaltspunkte, um über die Phrase $Я$ как пионер, всегда готов! (,Ich als Pionier bin für alles immer bereit!‘) die Implikatur ,Ich bin dafür!‘ zu ziehen. Daher liefert sie in \#3 den zweiten Teil der Phrase „vsegda gotov“ (всегда готов ,bin immer bereit'), die einerseits die floskelhafte Phrase komplettiert und somit die damit verbundene Implikatur offenlegt und andererseits auch im Falle der Nicht-Erschließung der Implikatur unabhängig davon als eine Zusage interpretiert werden kann.

Von den deutschen Reparaturinitiierungen wird ein Beispiel für die unter phrasalen und satzwertigen Reparaturmarkern größte Gruppe mit meinen (vgl. (112)) sowie ein Beleg für die satzwertige Reparaturinitiierung ich habe mich vertan (vgl. (113)) geliefert, die gleichzeitig das Bearbeiten von zwei pragmatischen Reparanda ankündigt.

Von sieben phrasalen und satzwertigen Reparaturinitiierungen mit meinen wird dieses in fünf Fällen in der 1. Person Singular Präsens (vgl. (112)) und in zwei Fällen in der 1. Person Singular Präteritum (vgl. (114)) verwendet. Im Gegensatz zu den bei Egbert (2009: 59) aufgeführten Beispielen mit der Initiierung im Format ich mein $+X$, wo $X$ für das Reparans steht, weisen sechs von sieben Reparaturinitiierungen in den analysierten Daten die invertierte Wortfolge auf, da das Vorfeld entweder durch das Reparans (vgl. (88)) oder durch das wiederholte Reparandum (vgl. (112)) besetzt wird (zu Konstruktionen mit meinen vgl. Imo 2007: 173-200): 
(112) Auszug aus dem SMS-Dialog \#3211:

Schreiberin $A$

Schreiberin $B$

$\rightarrow$

Ich bin nächsten Donnerstag in der Uni um 12 Uhr. Sollen wir vorher in

die Mensa? Vermiss dich! Wir müssen unbedingt was machen!!! :-)

Nachricht \#3-19.03.2014 - 08:55

$\rightarrow$

Also mit Dobnerstag meine ich morgen!!! :-)

Nachricht \#4-19.03.2014-08:55

soory ivh hab gestern an der filmanalyse gearbeitet... -.- voll vergessen

zu schreiben. ich bin an dem samstag in $b .{ }^{105}$ also dann morgen wie

viel uhr mensa? :D

Nachricht \#5 - 19.03.2014 - 09:21

Die in (112) vorliegende Reparatur richtet sich auf die Zeitreferenz „nächsten Donnerstag“ (\#3), die Schreiberin $A$ im Rahmen ihres Verabredungsangebots nennt. Der vorgeschlagene Termin wird von ihr jedoch anschließend korrigiert, indem sie die Zeitreferenz „nächsten Donnerstag“ mit „morgen“, also diesen Donnerstag, ersetzt (vgl. \#4). Dabei setzt $A$ zwei Reparaturmarker ein, nämlich „Also“ und „meine ich“, um ihre Korrektur kenntlich zu machen. Die explizite Wiederaufnahme eines Teils des Reparandums („mit Dobnerstag“106) erleichtert die Zuordnung des gelieferten Reparans. Die satzfinalen iterierten Ausrufezeichen erzeugen Emphase und unterstreichen die Relevanz der in \#4 übermittelten Information, während das Emoticon „:-)“ phatische Kommunikation markiert. $B$ 's Reaktion in \#5 ,also dann morgen wie viel uhr mensa?“ zeigt, dass $A$ 's Selbstreparatur von ihr wahrgenommen und richtig interpretiert wurde.

Von niedrigfrequenten Reparaturmarkern, die jeweils lediglich mit einem Token vertreten sind, soll auf die Reparaturinitiierung „ich hab mich vertan“ (\#12) eingegangen werden, die gleichzeitig zwei pragmatische Korrekturen einleitet:

105 Mit ,ich bin an dem samstag in b.“ lehnt Schreiberin $B$ A's Verabredungsvorschlag ab, am Samstag feiern zu gehen, der in \#2 (nicht abgebildet) geäußert wurde.

106 Der orthographische Fehler bei „Dobnerstag“ wird von $A$ jedoch nicht repariert, was auf unterschiedliche Gründe zurückgeführt werden kann. Zum einen ist es möglich, dass $A$ diesen Fehler nach dem Abschicken der Nachricht einfach nicht gemerkt hat. Zum anderen, wenn sie sich doch dessen bewusst war, dass in einer ihrer Nachrichten ein Fehler vorliegt, könnte sie diesen als nicht reparaturbedürftig einschätzen, da die gegenseitige Verständigung davon nicht beeinträchtigt wird. 
(113) Auszug aus dem SMS-Dialog \#1484:

Schreiberin $A$

Schreiberin $B$

Hi!sorry ass ich mich mehr gemeldet hab.die uni hat mich in ihren fesseln.wie wärs mitnächster woche Mittwoch?halb 5?lg

Nachricht \#10 - 13.07.2010 - 18:10:05

\begin{tabular}{c}
$\rightarrow \quad \begin{array}{c}\text { Sorry nächste woche mittwoch bin ich bei meinen großeltern } \\
\text { nachmittags, aber abends könnte ich. Sagen wir um 8? Das würde ich } \\
\text { schaffen! }\end{array}$ \\
\hline $\begin{array}{c}\text { Nachricht \#11 - 13.07.2010 - 20:34:05 } \\
\text { großeltern. Nächste woche mittwoch geht also. Wo sehen wir uns? :- }\end{array}$ \\
\hline Nachricht \#12 - 13.07.2010 - 20:45:05 \\
\hline $\begin{array}{c}\text { Ich schreib bis 4 klausur.danach kann ich hinkommen wohin du willst.je } \\
\text { nach wetter können wir auch schon draußen sitzen irgendwo.in } \\
\text { STADT3? }\end{array}$ \\
\hline Nachricht \#13 - 13.07.2010 - 20:52:05
\end{tabular}

Dem in (113) abgebildeten Dialog geht bereits eine Verabredung voraus, die aber von $B$ kurzfristig abgesagt wurde. Bei der sich direkt daran anschließenden Aushandlung eines neuen Termins teilt $B$ mit, dass sie ihre Planung in Bezug auf die Klausurvorbereitung noch durchdenken muss und sich daher später noch einmal bei $A$ meldet (nicht abgebildet). Erst eine Woche später löst $B$ ihr Versprechen ein und unterbreitet in \#10 einen neuen Terminvorschlag. Der von $B$ genannte Zeitpunkt wird zwar von $A$ abgelehnt, was sie mit einem geplanten Besuch rechtfertigt, sie schlägt aber gleichzeitig eine andere Zeit am gleichen Tag als Termin vor. In \#12 führt $A$ allerdings eine selbstinitiierte Selbstreparatur durch, indem sie die von ihr in \#11 erwähnte Zeitreferenz „nächste woche“ durch „erst in 2 wochen“ ersetzt. Die in \#12 erfolgte Korrektur der Zeitreferenz geht mit der pragmatischen Korrektur einher, da somit die von $A$ in \#11 geäußerte Ablehnung implizit zurückgenommen und durch eine explizite Zusage „Nächste woche mittwoch geht“ (\#12) mit also als Reparaturmarker ersetzt wird.

\section{(v) Kombinationen von expliziten Reparaturmarkern}

Explizite Reparaturmarker, die in Kap. 6.1.1.2 bisher beschrieben wurden, können auch miteinander kombiniert werden, was anhand von (95), (105) und (112) bereits gezeigt wurde. Bei verbalen Reparaturinitiierungen handelt es sich dabei 
meist um eine Kombination aus einer Partikel und einem phrasalen bzw. satzwertigen Reparaturmarker:

(114) Auszug aus dem Dialog \#2185:

Schreiber $A$

Schreiber $B$

\begin{tabular}{l}
$\rightarrow \quad$ Für wie viel soll ich den Gutschein holen? 10€? \\
\hline Nachricht \#9 - 13.02.2013- 20:03:05 \\
$\qquad \quad$ Ehm meinte 40€ \\
\hline Nachricht \#10 - 13.02.2013-20:03:05
\end{tabular}

Jup genau... Vorname und ich haben ja die platte für 20 geholt...

Bleiben also noch 40

Nachricht \#11 - 13.02.2013 - 20:03:05

Die Reparaturinitiierung, die im Kontext des Austausches bezüglich eines gemeinsamen Geschenks in \#10 erfolgt, schließt die Partikel „Ehm“ sowie den Reparaturmarker „meinte“ ein. Diese kündigen eine Korrektur an, die sich auf den Wert des Gutscheins „10€“ (\#9) bezieht, den $B$ besorgen soll. Im Laufe der Korrektur wird der falsche Betrag „10€“ durch „40€“ substituiert, wobei das $€$-Zeichen in dem Fall als postpositionierter syntaktischer Anker fungiert. Der als Reparans gelieferte Geldbetrag wird von $A$ in \#11 zunächst explizit mit „Jup genau...." und anschließend durch die erörterte Kalkulation bestätigt.

\subsubsection{Anbindung der Reparaturoperation}

Pfeiffer (2015: 262-263) weist darauf hin, dass Produzenten der Problemquelle im Rahmen der Reparaturdurchführung auf eine Reihe von Ressourcen zurückgreifen, die es dem Rezipienten erlauben, zum einen das Reparandum in der ursprünglichen Äußerung zu erkennen und zum anderen die durch die Reparaturdurchführung erzeugte „Veränderung in den Verlauf der ursprünglichen Äußerung [zu] integrieren“ (vgl. auch Fox/Jasperson 1995: 106). In diesem Zusammenhang spricht Pfeiffer (2015: 262-263) von der Anbindung der Reparaturoperation, die in erster Linie durch „die kategorielle Übereinstimmung von Teilen der ursprünglichen Äußerung und der Reparaturdurchführung“ gewährleistet wird, die die lexikalische, semantische oder syntaktische Ebene betreffen kann. Eine lexikalische Übereinstimmung liegt dann vor, wenn ein oder mehrere Wörter aus der ursprünglichen Äußerung bei der Reparaturdurchführung wiederholt werden, anders gesagt es handelt sich dabei um einen syntaktischen Anker (vgl. Pfeiffer 2015: 263). In den analysierten Daten findet sich der syntak- 
tische Anker bei zehn von 138 Reparaturen im deutschen und vier von 38 Reparaturen im russischen Korpus. Dabei weisen die deutschen Daten sechs Fälle mit präpositioniertem (vgl. \#4022 in der MoCoDa) und vier mit postpositioniertem syntaktischen Anker (vgl. (114)) auf, die zur Anbindung von acht Substitutionen, einer Insertion und einer Kombination aus einer Substitution und einer Insertion beitragen. In den russischen Dialogen lassen sich neben einem präpositionierten (vgl. (95)) und einem postpositionierten Anker (vgl. \#2812 in der $M o C o D a)$ zwei Fälle beobachten, in denen jeweils ein prä- und ein postpositionierter syntaktischer Anker zusammen auftreten (vgl. (103) und (110)). Während bei der Reparatur mit präpositionierten Ankern (vgl. (95)) eine Insertion durchgeführt wird, liegt in den drei anderen Fällen je eine Substitution vor.

Neben der lexikalischen Übereinstimmung zwischen der ursprünglichen Äußerung und der Reparaturdurchführung kann laut Pfeiffer (2015: 263) auch die syntaktische und/oder semantische Übereinstimmung zwischen Reparandum und Reparans als Hilfestellung bei der Anbindung der Reparaturoperation benutzt werden. Dabei baut diese Übereinstimmung auf der gleichen syntaktischen Funktion, die die beiden Konstituenten ausüben, oder auf der semantischen Relation zwischen ihnen auf. In (109) liegt z.B. insofern eine semantische Übereinstimmung zwischen dem Reparandum „Montag“ (\#3) und dem Reparans „Mittwoch“ (\#4) vor, da diese heteronym sind und zum Bedeutungsbereich Wochentage gehören.

Dadurch, dass alle analysierten selbstinitiierten Selbstreparaturen im next opportunity space medial schriftlich realisiert sind, bietet sich auch die partielle graphische Übereinstimmung des Reparandums und des Reparans als Mittel für die Anbindung der Reparaturoperation an. So weisen das Reparandum „Bein“ und das Reparans „Nein“ in (101) zwar weder syntaktische noch semantische Übereinstimmung auf, jedoch verfügen sie über einen identischen Wortauslaut, sodass der Rezipient der Problemquelle mithilfe dieser graphischen Ähnlichkeit den Bezug zwischen dem Reparandum und dem Reparans herstellen kann.

Bei in den untersuchten Daten auftretenden Insertionen wird eine weitere von Pfeiffer (2015: 263) beschriebene Ressource für die Anbindung der Reparaturoperation eingesetzt, nämlich die syntaktische Projektion:

(115) Auszug aus dem WhatsApp-Dialog \#4231

Schreiberin $A$ Schreiberin $B$

Linzer Torte ist ein Mürbeteig mit Zimt drin und dann kommt da Marmelade drauf und dann wieder Teigstreifen. Habe ich letztes Jahr einmal in Aachen gemacht. Ich kann dir ja mal ein Foto schicken

Nachricht \#7 - 19.11.2016 - 19:47 


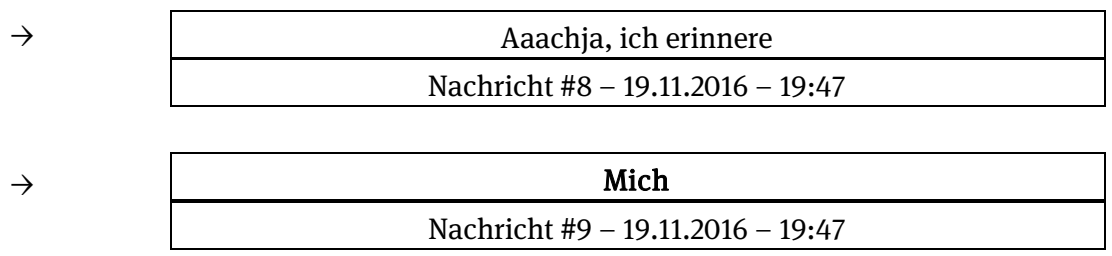

Nachricht \#7 bezieht sich auf die Frage danach, was eine Linzer Torte ist, die Schreiberin $B$ an ihre Mutter (Schreiberin $A$ ) in \#3 (nicht abgebildet) gestellt hat. In \#7 beschreibt $A$ die Zutaten und das Aussehen der Torte und verweist auf eine Situation, in der $B$ diese Torte gesehen hat. Danach bietet sie sogar an, ein Foto davon an $B$ zu schicken. Dieses Angebot lehnt $B$ jedoch in \#8 implizit ab. Das in \#9 vorliegende Reflexivpronomen „Mich“ lässt sich dank der Valenz des Verbs „erinnere“, das ein Akkusativobjekt oder ein Präpositionalobjekt projiziert $^{107}$, in Bezug auf \#8 als Insertion interpretieren.

Zur Anbindung der Reparaturoperation trägt m.E. auch die Wiederaufnahme des Reparandums bei (vgl. (104), (105) und (112)), die u.a. als die Reparaturinitiierung im Format nicht $X$ (vgl. Pfeiffer 2015: 303-304), wo $X$ für das Reparandum steht, realisiert wird. Dabei kann die Reparatur entweder dem Muster nicht Reparandum - Reparans (vgl. (106) sowie Pfeiffer 2015: 303) oder Reparans nicht Reparandum folgen, wie in (116) sowie \#3291 in der MoCoDa:

(116) Dialog \#2236:

\begin{tabular}{l}
$\rightarrow$ Schreiberin $A$ \\
\hline Weißt du schon wann du ungefähr wach bist :)? \\
\hline Nachricht \#1 - 09.07.2013 - 09:10:05 \\
\hline \\
\hline Da :D, Nicht wach :D \\
\hline Nachricht \#2 - 09.07.2013 - 09:37:05 \\
\hline wann passts dir am besten? :D \\
\hline Nachricht \#3 - 09.07.2013 - 11:25:05 \\
\hline also ich bin jetzt gleich Zuhause... \\
\hline Nachricht \#4 - 09.07.2013 - 11:35:05
\end{tabular}

$107 \mathrm{Vgl}$. die entsprechenden Wörterbucheinträge zum Verb erinnern in Wörterbuch zur Verbvalenz E-VALBU (https://grammis.ids-mannheim.de/verbs/view/400523/1) sowie auf grammis (https://grammis.ids-mannheim.de/verbvalenz/400523). 


\begin{tabular}{|c|}
\hline Ich kann kommen wann du willst ;) \\
\hline Nachricht \#5 - 09.07.2013-11:40:05 \\
\hline
\end{tabular}

so um 1 vielleicht :)?

Nachricht \#6-09.07.2013-11:41:05

\begin{tabular}{|c|}
\hline Ok :) \\
\hline Nachricht \#7 - 09.07.2013-11:42:05 \\
\hline
\end{tabular}

Die von $A$ in \#1 gestellte Frage enthält die Problemquelle „wach“, die sie in der darauffolgenden Nachricht im Rahmen einer selbstinitiierten Selbstreparatur bearbeitet. Dabei erscheint am Anfang der Nachricht, die die Reparaturinitiierung und -durchführung enthält, das Reparans „Da“, das mit dem nachgestellten Emoticon „:D“ kombiniert wird, dem eine evaluierende bzw. modalisierende Funktion (vgl. Pappert 2017: 195-198) zugeschrieben werden kann. Das negierte Reparandum „Nicht wach“, das ebenso mit einem Emoticon „:D“ versehen ist, wird nach dem Reparans platziert. Durch die somit erfolgte explizite Zurückweisung des Reparandums wird dem Rezipienten die Aufgabe erspart, dieses selbst lokalisieren zu müssen, was ihm bei der Verarbeitung der Reparatur behilflich sein kann (vgl. Pfeiffer 2015: 303-304).

\subsubsection{Reparaturdurchführung: Reparaturoperationen}

Bei der Betrachtung der Reparaturdurchführungen stellte sich heraus, dass Schreiber v.a. auf Substitutionen und Insertionen zurückgreifen, wobei die Substitutionen sowohl in den deutschen als auch in den russischen Daten häufiger als Insertionen vorkommen. Diese qualitative Verteilung kann zum Teil auch durch die Datenauswahl zustande gekommen sein, da man wegen der Tendenz zum Splitten einer Nachricht (vgl. Beißwenger 2007; Imo 2015a) in mehrere Nachrichten aus der Beobachterperspektive nicht immer erkennen kann, ob es sich in jedem konkreten Fall um eine Reparatur oder um eine bloße Expansion einer Nachricht handelt. Daher wurden für die Analyse nur solche Fälle herangezogen, bei denen man thematisch und/oder strukturell eine Reparatur erkennen konnte. Dabei lässt sich nicht ausschließen, dass einige Fälle, die vom Produzenten als Reparaturen konzipiert wurden - was sich aber aus der Beobachtersicht nicht erkennbar ist - nicht berücksichtigt wurden. Daher sollte man die quantitative Verteilung der Substitutionen und Insertionen mit Vorbehalt zur Kenntnis nehmen. 
Tab. 6: Reparaturoperationen im Untersuchungskorpus

\begin{tabular}{lll}
\hline Reparaturoperation & $\begin{array}{l}\text { Anzahl der Belege } \\
\text { im deutschen Subkorpus }\end{array}$ & $\begin{array}{l}\text { Anzahl der Belege } \\
\text { im russischen Subkorpus }\end{array}$ \\
\hline Substitution & 105 & 21 \\
Insertion & 32 & 17 \\
Tilgung & 1 & 1 \\
keine Reparaturdurchführung & 2 & 0 \\
(Metakommentar) & 1 & 0 \\
unklare Fälle & 141 & 39 \\
& &
\end{tabular}

Im Vergleich zu Substitutionen und Insertionen sind Tilgungen als Reparaturoperationen dagegen in den beiden Teilkorpora äußerst selten: Es wurden lediglich jeweils ein Beleg mit dieser Reparaturoperation in den deutschen und russischen Daten gefunden (vgl. \#1285 in der MoCoDa sowie (99)). Die Anzahl der Reparaturoperationen im jeweiligen Korpus ist in Tabelle 6 dargestellt.

\section{Substitutionen}

Bei Substitutionen in den analysierten Kurznachrichten fungieren meist Wörter (vgl. (88), (92) und (104)) als Reparantia, Phrasen bzw. Sätze (vgl. (89)) oder einzelne Zeichen (vgl. (117)) dagegen werden vergleichsweise seltener ersetzt:

(117) Auszug aus dem Dialog \#2059:

Schreiber $A$

Schreiber $B$

\begin{tabular}{|c|}
\hline moin moin \\
\hline Nachricht \#1 $-31.01 .2013-16: 01$ \\
\hline
\end{tabular}

$\rightarrow$

\begin{tabular}{|c|}
\hline Moisen, was gejt \\
\hline Nachricht \#2 $-31.01 .2013-16: 07$ \\
\hline
\end{tabular}

$\rightarrow$

\begin{tabular}{|c|}
\hline $\mathrm{h}$ \\
\hline Nachricht \#3 $-31.01 .2013-16: 07$ \\
\hline
\end{tabular}

heute abend ne runde pes??

Nachricht \#4 - 31.01.2013 - 16:09 


\begin{tabular}{|c|}
\hline mh lust schon, aber leider keine zeit \\
\hline Nachricht \#5 - 31.01.2013 - 16:10 \\
\hline
\end{tabular}

Das (ortho)graphische Reparandum „gejt“ aus \#2 könnte auch durch das Reparans geht substituiert werden. Dennoch entscheidet sich Schreiber $B$ für eine ,sparsamere‘ Reparaturdurchführung und liefert in \#3 lediglich einen einzelnen Buchstaben „h“, der anstelle von „;“ in der problematischen Verbform stehen soll.

Wenn man Substitution als Reparaturoperation breit fasst, könnte man diese auch auf Fälle übertragen, bei denen eine sprachliche Handlung durch eine andere ersetzt wird, wie dies in (118) der Fall ist:

(118) Dialog \#2234:

Schreiber $A$

Schreiber $B$

Jo, also NAME kommt gerade erst vom ORT und brauch ein bisschen

länger, sie würde uns 19:45 an der STELLE treffen wollen, aktzeptabel oder willste vorher alleine hinfahren ${ }^{\wedge \wedge}$

Nachricht \#1 - 12.07.2013 - 19:02:05

$\rightarrow$

Boah dann kommen wir doch voll spät man! :P

Nachricht \#2 - 12.07.2013-19:03:05

$\rightarrow$

Nee ist ok

Nachricht \#3 - 12.07.2013 - 19:03:05

\begin{tabular}{|c|}
\hline Ok alles klar, Perfekt \\
\hline Nachricht \#4 - 12.07.2013 - 19:05:05 \\
\hline
\end{tabular}

In (118) teilt Schreiber $A$ dem mit ihm befreundeten Schreiber $B$ eine Änderung der zuvor getroffenen Verabredung mit und bietet ihm zwei Alternativen an. Schreiber $B$ reagiert in \#2 auf den Vorschlag, sich doch vorher zu treffen, mit einem Vorwurf. Das Emoticon „:P“ gibt aber bereits einen impliziten Hinweis auf die scherzhafte Interaktionsmodalität der Nachricht, welche durch die in \#3 gelieferte ernsthafte Antwort verdeutlicht wird. Somit wird die nicht ernst gemeinte Antwort (\#2) zurückgenommen und durch eine Zustimmung der Planänderung ,ist ok“ substituiert (zur Verwendung von nee zum Kennzeichnen des Wechsels der Interaktionsmodalität vgl. Imo 2017: 64-65). 


\section{Insertionen}

Insertionen finden sich bei (ortho)graphischen Korrekturen, bei denen das fehlende Fragezeichen nachgereicht wird (vgl. (91)), sowie bei einer Reihe von lexiko-semantischen Reparaturen, v.a. bei denjenigen, in welchen es um die Präzisierung eines bereits gelieferten sprachlichen Ausdrucks geht (vgl. (95)). Des Weiteren können Insertionen vereinzelt bei syntaktischen Reparaturen beobachtet werden, bei denen das Einfügen einer Konstituente die Reihenfolge der Konstituenten in der ursprünglichen Nachricht ändert. Dabei wirkt sich die eingefügte Konstituente auch auf der lexiko-semantischen sowie pragmatischen Ebene der ursprünglichen Nachricht aus. Ein solcher Fall liegt in (119) vor, in dem Schreiberin $B$ dem mit ihr verwandten Schreiber $A$ einen Filmtipp gibt:

(119) WhatsApp-Dialog \#3964:

Schreiber $A$

Schreiberin $B$

\begin{tabular}{|c|}
\hline Interstellar! Muss du gucken \\
\hline Nachricht \#1 - 01.02.2016 - 17:42 \\
\hline
\end{tabular}

\begin{tabular}{|c|}
\hline Hatte ich längst im Kino geguckt $\sqrt{6 / 3}$ \\
\hline Nachricht \#2 $-01.02 .2016-17: 44$ \\
\hline
\end{tabular}

$\rightarrow$

Aber mir gefiel der leider nicht :( hatte mich voll gefreut auf den Film

Nachricht \#3 - 01.02.2016 - 17:45

$\rightarrow$

\begin{tabular}{|c|}
\hline Also damals gefiel der mir leider nicht so :/ \\
\hline Nachricht \#4-01.02.2016-17:45 \\
\hline
\end{tabular}

\begin{tabular}{|c|}
\hline Haha \\
\hline Nachricht \#5 - 01.02.2016 - 17:45 \\
\hline
\end{tabular}

\begin{tabular}{|c|}
\hline Ich liebe den Film 웡 \\
\hline Nachricht \#6 - 01.02.2016 - 17:48 \\
\hline
\end{tabular}

\begin{tabular}{|c|}
\hline Haha $\frac{}{6}$ \\
\hline Nachricht \#7 $-01.02 .2016-17: 52$ \\
\hline
\end{tabular}

Auf $B$ 's Filmempfehlung (\#1) geht $A$ in \#2 ein, indem er mitteilt, den Film bereits gesehen zu haben. Danach schildert $A$ seinen ersten, negativen, Eindruck von dem Film, den er dennoch in der nächsten Nachricht durch das ins Vorfeld 
eingefügte Adverb „damals“ relativiert. Somit wird seine für $B$ gesichtsbedrohende Bewertung abgeschwächt (zu Bewertungen im Kontext des face workvgl. Auer/Uhmann 1982), was die Möglichkeit offenlässt, dass sich seine Einstellung zum Film geändert hat.

\subsubsection{Mehrere Reparaturdurchführungen und Reparaturausgang}

Die Effizienz der in der interaktionalen Schriftlichkeit etablierten Reparaturpraktiken zeigt sich darin, dass in der überwiegenden Mehrheit der analysierten Fälle die Reparaturdurchführung, im ersten Anlauf' ihr Ziel erreicht und daher nach Egbert (2009: 70) als geglückt gelten kann. Im gesamten Korpus kommt es lediglich in zwei Dialogen dazu, dass die erste Reparaturdurchführung nicht erfolgreich ist und daher in Anlehnung an Egbert (2009: 70-72) als missglückt bezeichnet werden kann. Dabei wird in einem deutschen Beispiel (122a-b), welches in Kap. 6.1.1.6 näher betrachtet wird, ein zweiter Reparaturversuch unternommen, sodass die Reparaturdurchführung letztendlich doch gelingt. Im Gegensatz dazu veranschaulicht die in (120) dargestellte Reparaturdurchführung eine andere Option, mit misslungenen Reparaturen umzugehen, nämlich die Reparatur aufzugeben und auf den nächsten Reparaturversuch zu verzichten (vgl. auch Nachrichten \#6, 8, 9 in (122b)):

(120) Auszug aus dem Dialog \#2638:

Schreiber $A$

Schreiber $B$

\begin{tabular}{|c|}
\hline при том ЧТО \\
\hline wobei \\
\hline Nachricht \#5 - 08.12.2013-17:38:15 \\
\hline
\end{tabular}

$\rightarrow$

\begin{tabular}{|c|}
\hline у нас лекция почти нет \\
\hline wir so gut wie keine Vorlesung haben \\
\hline Nachricht \#6 - 08.12.2013 - 17:38:19 \\
\hline
\end{tabular}

$\rightarrow$

\begin{tabular}{|c|}
\hline лукций $^{\star}$ \\
\hline Vurlesungen $^{\star}$ \\
\hline Nachricht \#7-08.12.2013-17:38:24 \\
\hline
\end{tabular}

\begin{tabular}{|c|}
\hline да еп \\
\hline oh Mist \\
\hline Nachricht \#8-08.12.2013-17:38:29 \\
\hline
\end{tabular}




\begin{tabular}{|c|}
\hline ну ты понял \\
\hline du hast schon verstanden \\
\hline Nachricht \#9 $-08.12 .2013-17: 38: 31$ \\
\hline
\end{tabular}

\begin{tabular}{|c|}
\hline я я понел \\
\hline ich ich habe verstanden \\
\hline Nachricht \#10 $-08.12 .2013-17: 38: 36$ \\
\hline
\end{tabular}

Die Problemquelle in (120), in dem Schreiber $A$ seinem Freund $B$ über die Unterrichtssituation an seiner Universität berichtet, stellt das Nomen im Nominativ Singular „лекция“ (,Vorlesung“) aus \#6 dar, das aber in der vorliegenden syntaktischen Struktur im Genitiv stehen soll. Zudem macht den Gebrauch von „лекция“ (,Vorlesung') im Singular in dem Kontext wenig Sinn. Die beiden grammatischen Kategorien - Kasus und Numerus - versucht $A$ daher in \#7 zu korrigieren, indem er das Nomen „лекция“ (,Vorlesung-NOM.SG') grammatisch und semantisch an die Struktur der Nachricht \#6 anpasst und durch „лукций“ ersetzt. Das Reparans „лукций“ (,Lucius (der römische Vorname)') enthält dabei aber einen orthographischen Fehler im Stamm des Wortes, das eigentlich лекций (,Vorlesungen-GEN.PL') heißen soll. Allerdings unternimmt $A$ keinen weiteren Reparaturversuch, sondern reflektiert seine misslungene Reparatur mit „да еп“ (,oh Mist'; \#8) und liefert einen Account für das Ausbleiben des zweiten Reparaturversuchs „ну ты понял“ (,du hast schon verstanden`) in \#9. Schreiber $B$ geht auf den Account ein und liefert in \#10 eine explizite Verstehensbekundung „я я понел“ (,ich ich habe verstanden'), womit sich die Notwendigkeit der weiteren Reparatur erübrigt.

Während in (120) der zweite Anlauf der Reparaturdurchführung ausbleibt und stattdessen ein Metakommentar, der das eigene Schreibverhalten bewertet, sowie ein Account geliefert werden, greifen Schreiber in zwei weiteren deutschen Dialogen - (121) sowie (122c) - auf diese Strategie bereits beim ersten Reparaturversuch zurück:

(121) Auszug aus dem WhatsApp-Dialog \#3059:

Schreiber $A$

Schreiber $B$

\begin{tabular}{|c|}
\hline ich bin so ab 8 @ home \\
\hline Nachricht \#10 - 13.02.2014 - 14:05:05 ${ }^{108}$ \\
\hline
\end{tabular}

108 Dabei handelt es sich um die von der MoCoDa automatisch generierten Zeitstempel, die alle Nachrichten eines solchen Kurznachrichtendialogs erhalten, für den bei der Einstellung 


\begin{tabular}{|c|}
\hline dann kannse einfliegen \\
\hline Nachricht \#11 - 13.02.2014 - 14:05:05 \\
\hline
\end{tabular}

\begin{tabular}{|l|}
\hline aight, zurück wird eher gekrochen :D \\
\hline Nachricht \#12 - 13.02.2014 - 14:05:05 \\
\hline
\end{tabular}

\begin{tabular}{|c|}
\hline auf den brustwarzen :0 \\
\hline Nachricht \#13 - 13.02.2014 - 14:05:05 \\
\hline
\end{tabular}

\begin{tabular}{|c|}
\hline haha, du lappen \\
\hline Nachricht \#14 - 13.02.2014 - 14:05:05 \\
\hline
\end{tabular}

\begin{tabular}{|c|}
\hline wat denn :D \\
\hline Nachricht \#15 - 13.02.2014 - 14:05:05 \\
\hline
\end{tabular}

\begin{tabular}{|c|c|}
\hline \multirow[t]{2}{*}{$\rightarrow$} & BIN HALT NE MIMI UND VERTRAG NICHTS \\
\hline & Nachricht \#16 - 13.02.2014 - 14:05:05 \\
\hline
\end{tabular}

$\rightarrow$ UPS^^

\begin{tabular}{|c|}
\hline ALLES KLAR CAPTAIN CAPSLOCK :D \\
\hline Nachricht \#18-13.02.2014-14:05:05 \\
\hline
\end{tabular}

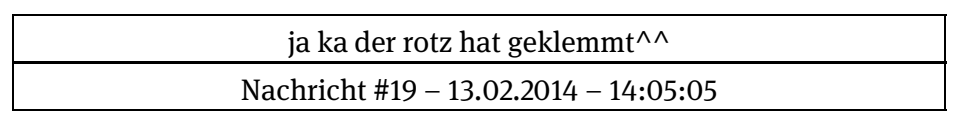

In \#10-11 teilt Schreiber $B$ seinem Freund mit, wann er bei ihm vorbeikommen kann. Das dabei von $B$ verwendete Verb „einfliegen“ (\#11) löst eine scherzhafte Kommunikation aus, in deren Rahmen Schreiber $A$ u.a. in Nachricht \#16 eine ironische Selbstbewertung abgibt. Diese Bewertung wird mit durchgehender Majuskelschreibung gestaltet, was in der interaktionalen schriftbasierten computervermittelten Kommunikation traditionellerweise als Ressource der emulierten Prosodie (Haase et al. 1997; Androutsopoulos 2007) für die Wiedergabe ,lauten Schreiens‘ bzw. generell als Emphasemarkierung eingesetzt wird. Die

der Daten in die Datenbank nicht eingetragen wurde, wann einzelne Nachrichten verschickt und empfangen wurden. 
direkt danach gelieferte Interjektion ups gibt zu erkennen, dass die Majuskelschreibung in dem vorliegenden Fall nicht intendiert war. Die - evtl. ebenso ungewollt - großgeschriebene Interjektion baut durch ihre graphische Gestaltung einen Bezug zur ungewünschten Majuskelschreibung in \#16 auf und drückt $A$ 's Einstellung zum eigenen Fehler aus. Schreiber $B$ nimmt $A$ 's Fehler als Anlass für einen Witz, indem er in \#18 auf $A$ mit „CAPTAIN CAPSLOCK“ referiert und die komplette Nachricht ebenso in konsequenter Großschreibung gestaltet. Daraufhin reagiert Schreiber $A$ in \#19 mit einer Erklärung für seinen Fehler, welcher in der Fehlfunktion der Taste lag, mit der die Schriftgröße eingestellt wird. Das Ausbleiben einer expliziten Reparaturdurchführung lässt sich in dem Fall v.a. durch die fehlende kommunikative Relevanz erklären. Durch $A$ 's Metakommentare in Nachrichten \#17 und \#19 erweist sich die konsequente Großschreibung in \#16 zwar als Problemquelle, da aber der Inhalt der Nachricht stimmt, ist eine Reparaturdurchführung, die in Form einer Substitution denkbar wäre, für beide Interaktionsteilnehmer hinfällig.

\subsubsection{Reaktionen auf selbstinitiierte Selbstreparaturen im next opportunity space}

In den meisten analysierten Dialogen wird die Kommunikation nach einer selbstinitiierten Selbstreparatur einfach fortgeführt und deren kommunikativer Erfolg durch die passende Reaktion des Rezipienten der Problemquelle implizit angezeigt (vgl. Deppermann/Schmitt 2008 zur impliziten Verstehensdokumentation). In vier Fällen im russischen und 19 im deutschen Subkorpus wird die bearbeitete Problemquelle bzw. die selbstinitiierte Selbstreparatur aber vom Rezipienten der Problemquelle auf eine bestimmte Weise thematisiert:

(i) Der Rezipient der Problemquelle kann sein Verstehen der durchgeführten Selbstreparatur explizit signalisieren (drei Fälle in den deutschen und ein Fall in den russischen Daten, vgl. (120)).

(ii) Der Rezipient kann zur durchgeführten Reparatur bzw. zum damit bearbeiteten Reparandum Stellung nehmen und diese bewerten, und zwar (a) mithilfe von Metakommentaren, (b) mit Emoticons und Emojis, wie :D, ^^, :P, $\Leftrightarrow$ etc., sowie (c) mit Lachpartikeln, wobei diese Ressourcen miteinander kombiniert werden können. In neun deutschen ,Reaktionsnachrichten“ liegen sechs Emoticons und Emojis (vgl. (122b-d)), fünf Lachpartikeln (vgl. (122b)) sowie drei Metakommentare (vgl. „lustig!“ in (123)) vor. Im russischen Subkorpus wird die Reparatur bzw. das Reparandum zwei Mal bewertet, wobei diese Bewertungen in Form eines Emoticons sowie eines Metakommentars in Kombination mit Emojis gestaltet sind (vgl. „Людоедка

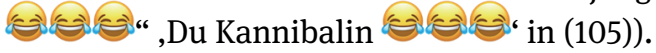


(iii) Der Rezipient der Problemquelle kann die Selbstreparatur oder das Reparandum als Anlass für eine Frotzelaktivität nutzen (ein Fall im russischen vgl. (99) - und sechs im deutschen Korpus - vgl. (97) sowie (122c)).

(iv) Der Rezipient der Problemquelle kann die vom Kommunikationspartner durchgeführte selbstinitiierte Selbstreparatur als unnötig bezeichnen (ein Fall in den deutschen Daten, vgl. \#4117 in der MoCoDa).

Die Reaktionsmöglichkeiten (i) bis (iii) lassen sich anhand des WhatsApp-Dialogs (122a-d) veranschaulichen, der mehrere selbstinitiierte Selbstkorrekturen enthält, die der aktivierten Autokorrekturfunktion geschuldet sind.

(122a) WhatsApp-Dialog \#4235:

Schreiberin $A$

Schreiberin $B$

\begin{tabular}{|c|}
\hline $\begin{array}{c}\text { Aber ich sage auch schon seit Jahren, dass ich ein Gedächtnis habe wie } \\
\text { Doris }\end{array}$ \\
\hline Nachricht \#1 - 05.10.2016 - 14:44 \\
\hline${ }^{\star}$ Doris \\
\hline Nachricht \#2 - 05.10.2016 - 14:45 \\
\hline
\end{tabular}

\begin{tabular}{|c|}
\hline Ja ich auch!!!! \\
\hline Nachricht \#3 $-05.10 .2016-14: 45$ \\
\hline
\end{tabular}

In der Einstiegsnachricht dieses Dialogs gibt Schreiberin $A$ eine ironisch-kritische Bewertung der eigenen Gedächtnisleistung ab und baut dabei einen Vergleich mit Dorie auf, einem unter Amnesie leidenden Fisch aus dem Animationsfilm Findet Dorie. Die Autokorrektur (vgl. A's Metakommentar in \#6) verwandelt aber den beabsichtigten Namen Dorie in einen anderen Namen „Doris“ (\#1) -, bei dem der intendierte Bezug zum Animationsfilm verloren geht. In \#2 unternimmt $A$ einen Korrekturversuch, der ihr aber nicht gelingt, da das Reparans „Doris“ lediglich eine Wiederholung des Reparandums darstellt. $A$ 's Fehler scheint allerdings keine Verstehensschwierigkeiten bei $B$ ausgelöst zu haben, da sie in \#3 eine sinnvolle Reaktion auf \#1 liefert.

(122b) WhatsApp-Dialog \#4235:

Schreiberin $A$

Schreiberin $B$

$\rightarrow \quad$ *dorie 60




\begin{tabular}{|c|}
\hline $\begin{array}{c}\text { Ich hab ein sieb mit einem großen loch drin im Kopf. } \\
\text { Also ein Ring }\end{array}$ \\
\hline Nachricht \#5 - 05.10.2016 - 14:45 \\
\hline
\end{tabular}

$\rightarrow \quad$ Doofe Autokorrelation $\Leftrightarrow$

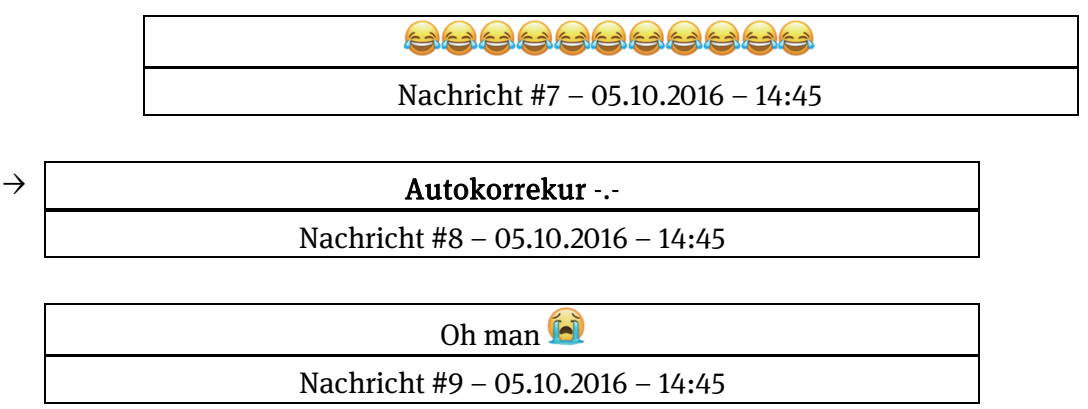

\begin{tabular}{|c|}
\hline Hahahahaha $\theta$ eी \\
\hline Nachricht \#10 $-05.10 .2016-14: 46$ \\
\hline
\end{tabular}

\begin{tabular}{|c|}
\hline Ich hasse T9 -0 \\
\hline Nachricht \#11 $-05.10 .2016-14: 46$ \\
\hline
\end{tabular}

Aber Autokorrelation klingt irgendwie so gehoben und fachlich Nachricht \#12 - 05.10.2016 - 14:46

\begin{tabular}{|c|}
\hline Is was dran $\theta=0$ \\
\hline Nachricht $\# 13-05.10 .2016-14: 46$ \\
\hline
\end{tabular}

Nach dem ersten missglückten Reparaturversuch unternimmt $A$ in \#4 einen zweiten, bei dem es ihr schließlich gelingt, den Namen - abgesehen von der initialen Großschreibung - richtig einzugeben. Ihre Frustration bringt sie zum einen mit dem das Reparans begleitenden Emoji $\because$ (\#4) und zum anderen mit dem anschließend verschickten Metakommentar (\#6) zum Ausdruck, in dem sie offenlegt, dass die Autokorrektur für das Entstehen des von ihr korrigierten Fehlers verantwortlich war. Allerdings wird das Wort Autokorrektur vom Texterkennungsprogramm ihres Smartphones zu „Autokorrelation“ (vgl. \#6) geändert, was eine Reaktion von $B$ in Form von iterierten Emojis (\#7) auslöst. Auf den missglückten Reparaturversuch in \#8 sowie auf den sich darauf 
beziehenden Metakommentar in \#9 reagiert $B$ zuerst mit der iterierten Lachpartikel in Kombination mit drei Emojis stimmt aber $A$ später auch zu, dass das Reparandum „Autokorrelation“ etwas an sich hat (\#13).

(122c) WhatsApp-Dialog \#4235:

\begin{tabular}{l} 
Schreiberin $A$ \\
\hline Hast du findet Doris auch schon gesehen? \\
\hline Nachricht \#14 - 05.10.2016 - 14:47 \\
\hline Oh schon wieder Doris 6 \\
\hline Nachricht \#15 - 05.10.2016 - 14:48 \\
\hline
\end{tabular}

\begin{tabular}{|c|}
\hline Kenn ich nicht den Film \\
\hline Nachricht \#16-05.10.2016 - 14:48 \\
\hline Nachricht \#17-05.10.2016-14:48 \\
\hline
\end{tabular}

\begin{tabular}{|c|}
\hline Ne, noch nicht. Du? \\
\hline Nachricht \#18 - 05.10.2016 - 14:48 \\
\hline
\end{tabular}

In \#14 kommt $A$ auf den Animationsfilm Findet Dorie zurück, auf den sie bereits in der ersten Nachricht implizit verwiesen hat. Dabei passiert ihr jedoch der gleiche Fehler wie in \#1 und \#2, da Dorie wieder in „Doris“ (\#14) umgewandelt wird. $A$ reflektiert zwar ihren Fehler in \#15, führt aber diesmal keine selbstinitiierte Selbstkorrektur durch, was zum einen daran liegen könnte, dass eine solche Korrektur bereits vor wenigen Nachrichten erfolgt ist, und zum anderen daran, dass $B$, wie \#3 und \#5 sowie im späteren Verlauf des Dialogs auch \#18 zeigen, trotzdem den Referenten erkannt hat. Allerdings zieht $B$ vor, anstatt eine ernsthafte Antwort auf die Frage aus \#14, ob sie den Film Findet Dorie gesehen hat, zu geben, ihre Freundin in \#16 zu frotzeln. Die Frotzelaktivität (vgl. Günthner 1996, 1999) kommt in dem Fall ähnlich wie in (97) dadurch zustande, dass $B$ die Problemquelle - den fehlerhaften Titel Findet Doris - nicht als solche behandelt und die Frage ernsthaft beantwortet, indem sie schreibt, dass sie den unter diesem falschen Titel nicht existierenden - Animationsfilm nicht kennt. Die scherzhafte Modalität ihrer Antwort markiert $B$ mit in \#17 verschickten iterierten Emojis. Zudem wird diese witzige Antwort auch durch eine ernste in \#18 abgelöst. 
(122d) WhatsApp-Dialog \#4235:

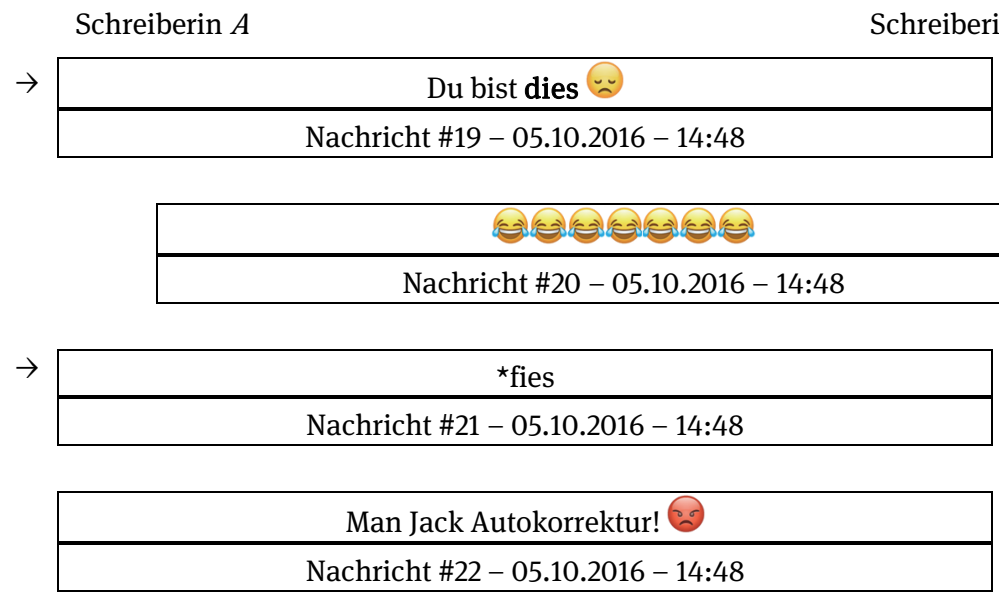

\begin{tabular}{|c|}
\hline Warum machst du plötzlich so viele Fehler? \\
\hline Nachricht $\# 23-05.10 .2016-14: 48$ \\
\hline
\end{tabular}

\begin{tabular}{|c|}
\hline Ich bin gerade so hart am lachen $\theta$ Nachricht \#24 - 05.10.2016-14:48 \\
\hline Nates \\
\hline
\end{tabular}

Die von $B$ in \#16 vollzogene Frotzelaktivität wird von $A$ vorwurfsvoll in \#19 zurückgewiesen. Erneut schlägt sich der Einfluss des Texterkennungsprogramms in dieser Nachricht von $A$ nieder, sodass „dies“ anstatt von fies verschickt wird. Die neue Problemquelle löst eine metakommentative Reaktion von $B$ aus, die zuerst in \#20 iterierte Emojis verschickt und nachher explizit eine Frage nach dem Grund für eine solche Anhäufung von Fehlern stellt, gefolgt von einer selbstreflexiven Bewertung von $A$ 's Schreibweise.

\subsubsection{Interaktionale Funktionen von selbstinitiierten Selbstreparaturen im next opportunity space}

Neben der primären Funktion von selbstinitiierten Selbstreparaturen im next opportunity space - nämlich der Prävention von Verstehens- und Verständigungsproblemen und der Aufrechterhaltung der Intersubjektivität (vgl. Stukenbrock 2013: 241-242) - könnte man einzelne Reparaturen in den analysierten Kurznachrichtendialogen als interaktionale Ressourcen sehen, die entweder zur Wahrung des Gesichts des Produzenten der Problemquelle (vgl. face work im 
Sinne von Goffman 1967) beitragen oder für die Gestaltung einer Frotzelaktivität (vgl. Günthner 1996, 1999) eingesetzt werden. Die gesichtsschonende Funktion könnte beispielsweise denjenigen Reparaturen zugeschrieben werden, bei denen (ortho)graphische Reparanda bearbeitet werden, obwohl diese im Grunde genommen das Verstehen der gesamten Nachricht nicht beeinträchtigen. Durch eine solche Selbstkorrektur kann der Produzent der Problemquelle sich als kompetenter Schreiber präsentieren, der eigene Fehler erkennen und korrigieren kann. Allerdings weist Markman (2006: 135-136) darauf hin, dass sich die Anzahl der Korrekturen, die sich auf (ortho)graphische Problemquellen richten, damit zu tun zu haben scheint, wie lange die Schreiber im Kontakt miteinander - und vermutlich auch davon, in welcher Beziehung zueinander - stehen. Dabei wird im Laufe der Zeit eine Art gruppenspezifische Norm entwickelt in Bezug darauf, welche Problemquellen bearbeitet und welche toleriert werden, sodass der Reparaturmechanismus zudem auch eine gewisse soziale Funktion ausübt (vgl. Markman 2006: 135-136).

Bei vier selbstinitiierten Selbstreparaturen aus den deutschen Daten, die als Mittel für eine Frotzelaktivität benutzt werden, wird eine scherzhafte, aber als ernst gemeint präsentierte sprachliche Handlung geliefert, die danach durch eine tatsächlich ernste Antwort abgelöst wird, sodass der Wechsel der Interaktionsmodalität stattfindet (vgl. auch (118) und (122c)). Die Problemquellen - wie die Ablehnung einer Bitte als eine dispräferierte sprachliche Handlung in (123) werden somit im Gegensatz zu anderen Reparanda im Korpus absichtlich erzeugt:

(123) Dialog \#1229:

Schreiber $A$

Schreiber $B$

Wer bock hat kann um 16 uhr gerne zum aufbauen vorbeikommen ;)

Nachricht \#1 - 14.10.2011 - 12:34

$\rightarrow$

Fahre leider erst um vier zurück nach ORT...daher werden wir uns erst heute abend zum feiern sehen

Nachricht \#2 - 14.10.2011 - 12:54

$\rightarrow$

War natürlich n witz...;) bin gleich auf jeden fall da...vielleicht komm ich etwas später,aber vor halb fünf müsste ichs eigentlich schaffen.

Nachricht \#3 - 14.10.2011 - 12:56 
lustig! :) is auch nich mehr viel was gemacht werden muss...also lass dir ruhig zeit. bis gleich dann

Nachricht \#4 - 14.10.2011 - 13:08

Schreiber $A$, der an jenem Abend seinen Geburtstag feiert, spricht seinem Freund $B$ in $\# 1$ eine subtile Bitte aus, ihm beim Aufbau zu helfen. Diese Bitte wird jedoch von $B$ zuerst abgelehnt, wobei die Ablehnung auch plausibel rechtfertigt wird, sodass die in \#2 vorliegende Antwort als ernsthaft wahrgenommen werden kann. Erst durch den mit „War natürlich n witz...;)“ explizit markierten Interaktionsmodalitätswechsel wird die Ablehnung zurückgezogen und durch die anschließende Zusage ersetzt.

\subsubsection{Selbstinitiierte Selbstreparaturen im same turn}

\subsubsection{Reparanda}

\section{Lexiko-semantische Reparanda}

Im Rahmen der untersuchten selbstinitiierten Selbstreparaturen im same turn werden überwiegend lexiko-semantische sowie pragmatische Problemquellen bearbeitet. ${ }^{109} \mathrm{Zu}$ lexiko-semantischen Reparaturen zählen z.B. diejenigen, bei denen die Bedeutung eines aus der Sicht des Produzenten für den Rezipienten unbekannten Lexems erklärt wird (vgl. (125)). In (124) wird eine semantische Elaborierung unternommen, um einen bereits gelieferten sprachlichen Ausdruck durch einen anderen, im Kontext passenderen bzw. präziseren zu ersetzen:

109 In den deutschen Analysedaten liegen 60 selbstinitiierte Selbstreparaturen im same turn vor, 33 von denen pragmatische und 24 lexiko-semantische Problemquellen bearbeiten. Des Weiteren weist das deutsche Subkorpus fünf syntaktische und eine (ortho)graphische selbstinitiierte Selbstreparaturen im same turn auf. Neben pragmatischen (6 Fälle) und lexiko-semantischen selbstinitiierten Selbstreparaturen (8 Fälle) im same turn findet sich in den russischen Daten lediglich ein einziger Fall, in dem sich die Reparatur auf ein syntaktisches Reparandum richtet. Es muss darauf hingewiesen werden, dass eine Reparatursequenz aus dem russischen und drei aus dem deutschen Subkorpus Problemquellen enthalten, die jeweils zwei verschiedenen sprachlichen Ebenen zugeordnet wurden. 
(124) Auszug aus dem Dialog \#2622:

Schreiberin $B$

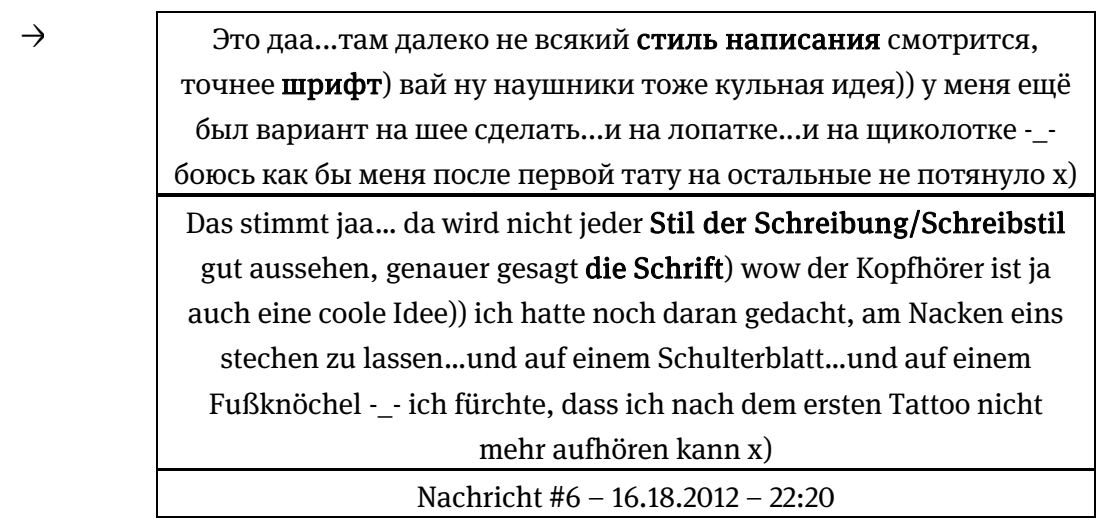

In (124) berichtet Schreiberin $B$ ihrem Kommunikationspartner darüber, dass sie sich einen lateinischen Spruch als Tattoo stechen lassen möchte. Schreiber $A$ evaluiert diese Idee positiv, räumt aber ein, dass das Tattoo gut gemacht werden muss, um cool auszusehen (nicht abgebildet). Schreiberin $B$ schließt sich in \#6 dieser Meinung an (,Это даа...“, Das stimmt jaa...') und merkt an, dass für ein Tattoo in lateinischer Schrift nicht jede graphische Gestaltung („стиль написания“", wortwörtlich ,Stil der Schreibung') passend ist. Die Nominalphrase „стиль написания“ (,Stil der Schreibung') als Kollokation bezieht sich aber im Russischen in erster Linie auf den Schreibstil eines Textes, während die Bedeutung ,Schriftgattung' bzw. ,graphische Gestaltung des Textes', die vermutlich hier aktiviert werden müsste, die dem Terminus „стиль написания“ in der Typographie oder Kalligraphie zugeschrieben wird, nicht unbedingt von einem Laien verstanden werden kann. Die in (124) von $B$ vorgenommene Substitution der Nominalphrase „стиль написания“ (,Stil der Schreibung) durch das Nomen „шрифт“ (,Schrift') dient somit entweder der Disambiguierung der Problemquelle oder - falls der Rezipient sich der zweiten Bedeutung von „стиль написания“" (,Stil der Schreibung') nicht bewusst ist - der Substitution eines evtl. unbekannten Fachbegriffs durch ein Lexem, das auch außerhalb der Fachsprache gut verständlich ist. Der Reparaturmarker „точне““(,genauer (gesagt)`) gibt in dem Fall einen Hinweis darauf, dass es sich dabei um eine semantische Elaborierung handelt.

Als lexiko-semantisches Reparandum kann außerdem ein Lexem angesehen werden, dessen Bedeutung aus der Sicht des Produzenten insofern als ,reparaturbedürftig“ (Stukenbrock 2013: 242) gilt, da sie dem Rezipienten u.U. unbekannt ist und daher ein potenzielles Verstehensproblem bereiten könnte: 
(125) Auszug aus dem WhatsApp-Dialog \#4228:

Schreiberin $A$

Schreiberin $B$

Hey! Wie geht es dir? Sag mal hast du eigentlich schon Pläne für Silvester?

Nachricht \#1 - 02.12.2016 - 10:23:20

Hey Puni :) Bin ab morgen in Oldenburg! Macht ihr was am We? Noch keine speziellen...Habt ihr ne coole Idee?

Nachricht \#2 - 02.12.2016 - 11:55:25

Am 1.1 abends fahre ich halt in den Skiurlaub...

Nachricht \#3 - 02.12.2016 - 11:55:40

Ich bin morgen auf einem Geburtstag von einer bekannten. Ich weiss

nicht wie es bei den anderen aussieht.

Nachricht \#4 - 02.12.2016 - 12:00:00

$\rightarrow$

Es gibt Grade die Überlegung vielleicht Krimidinner zu machen. Also man kocht was schönes jeder bekommt eine Rolle.

Nachricht \#5 - 02.12.2016 - 12:00:10

\begin{tabular}{|c|}
\hline Ah ok und wo das ganze? \\
\hline Nachricht \#6 - 02.12.2016 - 12:11:25 \\
\hline
\end{tabular}

Die Idee ist schön! Man müsste halt früh anfangen bzw eigentlich können wir ein Krimidinner auch einfach am 30.12 oder 29.12 zum Beispiel machen :)

Nachricht \#7 - 02.12.2016 - 12:14:30

Während der Besprechung ihrer Pläne für Silvester skizziert Schreiberin $A$ in \#5 den Vorschlag, ein Krimidinner zu organisieren. Danach erläutert sie, was zu dieser Freizeitaktivität gehört, nämlich ein Abendessen sowie ein Rollenspiel. Der in \#6 von $B$ realisierte Erkenntnisprozessmarker „Ah“ bezieht sich allerdings eher auf die von $A$ geäußerte Idee als auf die in der gleichen Nachricht platzierte Reparatur. Diese Interpretation wird v.a. davon gestützt, dass $B$ in \#11 (nicht abgebildet) mitteilt, dass sie bereits bei einem Krimidinner mitgemacht hat. 


\section{Pragmatische Reparanda}

Pragmatische Reparanda bei same turn repairs schließen diverse Problemquellen ein, darunter auch diejenigen, bei denen eine problematische Referenz z.B. eine mit dem Personalpronomen wir zum Ausdruck gebrachte Personenreferenz in (126) - im Rahmen einer prospektiven selbstinitiierten Selbstreparatur präzisiert wird:

(126) Auszug aus dem Dialog \#2062:

Schreiberin $A$

Schreiberin $B$

Hey NAME, Herzlichen Glückwunsch zum Geburtstag wünschen wir dir

:) Also NAME und ich! Würd dich auch anrufen, aber weiß nicht ob du

Uni hast oder nicht. Ich hoffe dir geht es gut. Freuen uns schon auf morgen und Samstag :)

Nachricht \#1 - 04.12.2012 - 12:51

$\begin{gathered}\text { Hey NAME vielen lieben dank für eure sms gestern :-)) Ich freue mich } \\ \text { auch schon auf samstag wenn ihr alle kommt. Weis noch nicht ob ich } \\ \text { heute mitkomme zum weihnachtsmarkt würde gerne zum chor gehen lg } \\ \text { und bis samstag auf jedenfall NAME :) }\end{gathered}$
Nachricht \#2-05.12.2012 - 12:15

Das in (126) vorliegende Reparandum stellt eine problematische Personenreferenz (,wir“ in \#1) dar, mit der Schreiberin $A$ Bezug auf Gratulanten einschließlich sich selbst nimmt. Obwohl Schreiberin $A$ theoretisch diese Problemquelle bereits vor dem Abschicken der Nachricht im Laufe einer Revision beseitigen könnte, zieht sie es jedoch vor, die Bearbeitung des deiktischen Personalpronomens für den Rezipienten sichtbar zu machen. Dafür leitet sie erst im Anschluss an den syntaktisch abgeschlossenen Teil der Nachricht mit Geburtstagsgrüßen, deren Ende mit dem Emoticon „:)“ markiert wird (vgl. strukturierende Funktion bei Pappert 2017: 198), eine selbstinitiierte Selbstreparatur ein. Der Reparaturinitiierung in Form des Diskursmarkers „Also“ folgt die Reparaturdurchführung, in der der unklare deiktische Referenzausdruck mit einer Kombination aus einem Namen und dem Personalpronomen „ich“ gleichgesetzt wird.

Neben problematischen deiktischen Ausdrücken richten sich pragmatische Selbstreparaturen in den untersuchten Daten auch auf die in einer Nachricht vollbrachten sprachlichen Handlungen, indem eine sprachliche Handlung zurückgezogen und durch eine andere ersetzt wird: 
(127) Auszug aus dem SMS-Dialog \#2315:

Schreiberin $A$

Schreiber $B$

\begin{tabular}{|c|}
\hline Warum sie nicht auf mein Anruf antworten der Herr??? \\
\hline Nachricht \#1 - 27.08.2013 - 16:22 \\
\hline
\end{tabular}

$\rightarrow$

Hä, ich red doch nicht mit jedem :) Nee, wollte gleich zurück rufen. Echt :)

Nachricht \#2-27.08.2013-16:30

Laber net!!! Wolltest du gar nicht. Dir kann man echt nicht vertrauen :)

Nachricht \#3 - 27.08.2013 - 16:41

Nachdem Schreiber $B$ einen eingehenden Anruf von $A$ nicht angenommen hat, verschickt Schreiberin $A$ Nachricht \#1 mit einem Vorwurf. $B$ weist $A$ 's Vorwurf zunächst in scherzhafter Interaktionsmodalität zurück, welche mit dem Emoticon „:)“ markiert wird (vgl. Imo 2015b: 148-150). Durch die darauffolgende Partikel „Nee“ wird diese Zurückweisung allerdings außer Kraft gesetzt und durch den Account „wollte gleich zurück rufen“ ersetzt. Die weitere Kommunikation verläuft ebenso in scherzhafter Modalität.

Des Weiteren lässt sich jeweils eine Reparatur aus dem russischen und deutschen Korpus (vgl. (128) sowie \#1273 in der MoCoDa), die eine Relativierung einer extreme case formulation im Sinne von Pomerantz (1986) darstellen, ebenso als pragmatisch ansehen:

(128) SMS-Dialog \#2637:

Schreiberin $A$

Schreiberin $B$

\begin{tabular}{|c|}
\hline [женское имя] купила мне подарок, но какой, я не знаю, а т.к. \\
я и тебя и ей заказала диснеееей^^, уточни, пожалуйста \\
:)))С любовью и поцелуями, донельзя деловая я :DD Как ты? \\
\hline [Frauenname] hat ein Geschenk für mich gekauft, aber ich weiß nicht \\
was für eins, da ich mir aber von euch beiden Disney gewünscht \\
habe^^, frag sie bitte am besten selbst :))) Mit Liebe und Kuss deine \\
Äußerst-Praktische :DD Wie geht es dir? \\
\hline Nachricht \#1 - 17.12.2010 - 19:28 \\
\hline
\end{tabular}




\begin{tabular}{|c|}
\hline $\begin{array}{c}\text { Ыыы))) окэ)я как...сопливо температурно...больногорлово О_о вот } \\
\text { как -_- }\end{array}$ \\
\hline $\begin{array}{c}\text { Hahaha))) Ok) wie es mir geht...schniefend fiebernd...halsschmerzend } \\
\text { O_o irgendwie so -__- }\end{array}$ \\
\hline Nachricht \#2 - 17.12.2010 - 19:31
\end{tabular}

$\rightarrow$\begin{tabular}{c|} 
Хоулэщет,дружище,ю маст ту кьюр йоселф.Немедля!))Ну как \\
минимум к среде) $)^{110}$ \\
\hline Holy shit meine Liebe, you must to cure yourself. Sofort!)) Na ja \\
zumindest bis Mittwoch) \\
\hline Nachricht \#3 - 17.12.2010 - 19:32 \\
\hline
\end{tabular}

\begin{tabular}{|c|}
\hline Я приду в понедельник) \\
\hline Ich komme am Montag) \\
\hline Nachricht \#4 - 17.12.2010 - 19:39 \\
\hline
\end{tabular}

\begin{tabular}{|c|}
\hline Молодец :) \\
\hline Braves Mädchen :) \\
\hline Nachricht \#5 - 17.12.2010 - 19:45 \\
\hline
\end{tabular}

\begin{tabular}{|c|}
\hline Ыы)ай ноу ай ноу :D \\
\hline Haha) I know I know :D \\
\hline Nachricht \#6 - 17.12.2010 - 19:50 \\
\hline
\end{tabular}

In (128) weist Schreiberin $A$ ihre Freundin zuerst darauf hin, dass diese sich vor dem Besorgen eines Geschenks für $A$ mit einer dritten Person absprechen muss, um nicht das gleiche Geschenk zu kaufen. Dann fragt $A$, wie es $B$ geht, woraufhin $B$ kreativ beschreibt, dass sie krank ist, indem sie ihre Symptome als eine Liste von okkasionell gebildeten Kurzformen der Adjektive liefert, welche morphologisch von Nominalphrasen abgeleitet wurden, die sowohl aus einem No-

110 Dabei fällt auf, dass in \#3 ein Phänomen vorliegt, welches Ma (i.Dr.) als Code-Switching ohne Script-Switching bezeichnet. Der erste Teil der Nachricht wird fast ausschließlich auf Englisch und der zweite auf Russisch verfasst. Dadurch, dass für der Nachrichtenteil auf Englisch nicht die entsprechende lateinische Schrift benutzt wird, sondern diese mithilfe des russischen kyrillischen Alphabets abgebildet wird, findet aber kein Wechsel auf der Schriftebene statt. 
men (сопли (,Popel, Schnupfen') $\rightarrow$ сопливо (,schniefend') ${ }^{111}$, температура (,Temperatur, Fieber') $\rightarrow$ температурно (,fiebernd')) als auch aus einem Nomen und einem Adjektiv (больное горло (,schmerzender Hals') $\rightarrow$ больногорлово (,halsschmerzend')) bestehen. Darauf reagiert $A$ mit einer Aufforderung an $B$, sich sofort („Немедля!“ ,sofort, auf der Stelle) auszukurieren, in deren Rahmen allerdings ein Sprachenwechsel (vgl. Fußnote 110) aus dem Englischen ins Russische erfolgt, dem man eine gesichtsschützende Funktion zuschreiben kann. Die Dringlichkeit von B's Genesung (vgl. extreme case formulation bei Pomerantz 1986) wird allerdings innerhalb derselben Nachricht relativiert, indem realistische Fristen dafür gesetzt werden („Ну как минимум к среде“ „Na ја zumindest bis Mittwoch'). ${ }^{112}$

\subsubsection{Reparaturinitiierungen}

\section{Reparaturinitiierungen ohne explizite Reparaturmarker}

Im Unterschied zu Reparaturen im next opportunity space, die in den beiden Sprachen in (über) 50\% der Fälle ohne explizite Reparaturmarker realisiert werden, lässt sich bei selbstinitiierten Selbstreparaturen im same turn eine entgegengesetzte Tendenz beobachten: Lediglich zwölf Prozent russischer und acht Prozent deutscher selbstinitiierter Selbstreparaturen im same turn weisen keine expliziten Reparaturmarker auf. ${ }^{113}$ Die Tatsache, dass explizite Reparaturmarker somit häufiger bei Reparaturen im same turn als im next opportunity space eingesetzt werden, hängt höchstwahrscheinlich damit zusammen, dass, wenn die Problemquelle und die Reparaturdurchführung innerhalb einer Nachricht liegen und sogar u.U. direkt aufeinander folgen, die Notwendigkeit, die ,Übergangsstelle` zur Reparaturdurchführung explizit zu markieren, stärker ist, als wenn sich die Problemquelle und die Reparaturdurchführung in unterschiedli-

111 Das Wort „сопливо“ existiert zwar in der russischen Sprache, wird aber im Gegensatz zu dessen Gebrauch in (128) in übertragener Bedeutung verwendet und bedeutet entweder ,seifenopermäßig، oder (in Bezug auf eine Leistung), nicht gut, zu schwach‘.

112 In einem vergleichbaren Beispiel aus dem deutschen Subkorpus (vgl. \#1273 in der MoCoDa) nimmt die selbstinitiierte Selbstreparatur in Nachricht \#3 eine ähnliche Form an: „Dann weck ihn es ist LEBENSWICHTIG!!!!!Zumindest ist es für mich wichtiger als der schlaf von [NAME].lol. :) usw.“. Dabei tragen die Majuskelschreibung sowie die iterierten Ausrufezeichen als graphostilistische Ressourcen zur Konstitution einer extreme case formulation im Sinne von Pomerantz (1986) bei. Die Relativierung des Wichtigkeitsgrads von A's Anliegen wird mit „Zumindest“ eingeleitet, das im Prinzip dem russischen „как минимум“ entspricht.

113 Den Prozentangaben entsprechen folgende absolute Zahlen: zwei von 14 russischer und fünf von 60 deutscher selbstinitiierter Selbstreparaturen im same turn. 
chen Beiträgen befinden und somit bereits durch die Nachrichtengrenze voneinander abgetrennt sind. Darüber hinaus lassen sich einzelne Reparaturen - v.a. Substitutionen - innerhalb derselben Nachricht nicht ohne vorherige ,Ankündigung، in Form einer Reparaturinitiierung durchführen. Dabei ist anzumerken, dass untersuchte selbstinitiierte Selbstreparaturen im same turn abgesehen von drei Belegen aus dem deutschen Subkorpus ausschließlich Elaborierungen darstellen, sodass das unmittelbare Aufeinandertreffen des Reparandums und des Reparans in solchen Fällen u.U. nicht als Reparatur erkannt werden kann.

Falls in selbstinitiierten Selbstreparaturen im same turn in einigen wenigen Fällen kein expliziter Reparaturmarker vorliegt, werden das Reparandum und die Reparaturdurchführung durch Interpunktionszeichen (vgl. (129)) oder durch Emoticons, die eine strukturierende Funktion ausüben (vgl. Pappert 2017: 198), voneinander abgegrenzt:

(129) Auszug aus dem Dialog \#1961:

Schreiberin $A$

Schreiberin $B$

\begin{tabular}{|c|}
\hline Wieder im lande :) \\
\hline Nachricht \#1 - 19.10.2012 - 20:20 \\
\hline
\end{tabular}

\begin{tabular}{|c|}
\hline Uuuund?:-) \\
\hline Nachricht \#2 - 19.10.2012 - 20:35 \\
\hline
\end{tabular}

\begin{tabular}{|c|}
\hline Sehr gut mit viel Sonnenschein erholt ;) \\
\hline Nachricht \#3 - 19.10.2012 - 20:35 \\
\hline
\end{tabular}

$\rightarrow$

\begin{tabular}{|c|}
\hline Das freut mich:-) was macht der Tiger? Der 4-beinige;-) \\
\hline Nachricht \#4-19.10.2012 - 20:35 \\
\hline
\end{tabular}

War die Zeit bei meinen Eltern und mein Papa will den gar nicht mehr hergeben :D

Nachricht \#5 - 19.10.2012 - 20:37

\begin{tabular}{|c|}
\hline Verständlich:-) \\
\hline Nachricht \#6 - 19.10.2012 - 20:38 \\
\hline
\end{tabular}

In \#1 kündigt Schreiberin $A$ ihre Rückkehr aus dem Auslandsurlaub an, woraufhin $B$ in \#2 einen Bericht darüber anfordert. Nachdem $A$ ihren positiven Eindruck vom Urlaub mitgeteilt hat, äußert $B$ ihre Freude und stellt die nächste Frage. Dabei erkundigt sie sich nach ,dem Tiger‘, fügt aber hinzu, dass sie den 
vierbeinigen Tiger meint. Die Insertion „4-beinige“ erhält ihren elaborierenden Charakter insofern, dass sie eine beabsichtigte Ambiguität der Referenz „der Tiger“ (\#4), womit auch der Freund von $A$ gemeint sein konnte, mit dem sie den Urlaub gemacht hat und welcher später im Dialog erwähnt wird, auflöst. Eine temporäre Ambiguität, die durch die Insertion allerdings aufgehoben wird, trägt zur Konstruktion der scherzhaften Interaktionsmodalität bei, welche mit dem Emoticon ,;-)“ gekennzeichnet wird.

\section{Nonverbale Reparaturinitiierungen}

$\mathrm{Zu}$ nonverbalen Ressourcen, die bei selbstinitiierten Selbstreparaturen im same turn eine Rolle spielen, können Klammern, Auslassungspunkte sowie die Majuskelschreibung gezählt werden.

Im Gegensatz zu den in Kap. 3.4.2.1 beschriebenen Funktionen von Klammern in der Kommunikation via Internet Relay Chat, bei denen ein einzelnes Lexem bzw. das Ausbleiben einer Phrase als Problemquelle auf der (ortho)graphischen, lexiko-semantischen oder morphosyntaktischen Ebene fungieren, handelt es sich bei der Klammerverwendung in den hier analysierten Daten eher um das Abgrenzen von Metakommentaren vom Rest der Nachricht. Diese Metakommentare haben dabei einen elaborierenden Charakter, da sie Hinweise zur Interpretation bzw. Einordnung der davorstehenden sprachlichen Handlungen geben:

(130) SMS-Dialog \#1882:

Schreiberin $A$

Schreiberin $B$

hi [vorname] ich hab mich heute mit [männername] getroffen aber er fragt mich nie was, bin echt ratlos.. lg [spitzname]

Nachricht \#1 - 08.02.2012 - 19:48:24

Hi, schön von dir zu hören. Wie ist es denn zu dem treffen gekommen? Fragst du ihn denn etwas? Nachricht \#2 - 08.02.2012 - 21:22:11

musst du eigentlich viel arbeiten oder hast du mal zeit für ein getränk? (an dich nicht an [männername] gerichtet) ja klar ich frag ihn voll viel aber ich weiß nie woran ich bei ihm bin.. wir waren frühstücken und auf dem aasee spazieren weil ich gefragt hatte, von ihm käm so ein vorschlag nicht! :P

Nachricht \#3 - 08.02.2012 - 21:25:32 
Ich hab auch mal zeit für ein Getränk ;) hast du mal darüber nachgedacht ihm einfach zu sagen was Sache ist? Das ist doch kein zustand so

Nachricht \#4 - 08.02.2012 - 21:39:32

In (130) berichtet Schreiberin $A$ über ein Treffen mit einem Mann, den $A$ und $B$ kennen, und beschwert sich bei $B$ darüber, dass dieser keine Fragen an sie während ihrer Treffen stellt. In diesem Zusammenhang erkundigt sich $B$ darüber, ob $A$ die Initiative in der Gesprächsführung übernommen hat und diesen Mann mal selbst nach etwas gefragt hat (vgl. \#2: „Fragst du ihn denn etwas?“). Als Antwort darauf kommt eine längere Nachricht, die aber mit einer Frage beginnt, welche man in Anbetracht der vorherigen Nachricht als Frage an den Mann, mit dem sich $A$ getroffen hat, interpretieren könnte. Mithilfe der in Klammern eingeschlossenen Erläuterung „(an dich nicht an [männername] gerichtet)“ (\#3) ergreift $A$ eine präventive Maßnahme und expliziert, wen sie mit der Frage adressiert, nämlich Schreiberin $B$ und nicht den Mann, der in \#1 und \#2 erwähnt wurde. Während in den deutschen Daten lediglich in (130) auf die Klammern zurückgegriffen wird, treten in den russischen Kurznachrichtendialogen zwei solcher Fälle auf (vgl. z.B. \#3276 in der MoCoDa).

Ein weiteres für selbstinitiierte Selbstreparaturen im same turn relevantes nicht-alphabetisches Zeichen stellen Auslassungspunkte dar (fünf Fälle im russischen und drei im deutschen Subkorpus; zu Funktionen von Auslassungspunkten in der WhatsApp-Kommunikation vgl. Busch 2021: 378-406). Das trifft z.B. für Dialog \#2622 aus den russischen Daten zu, der durch die überdurchschnittlich langen Nachrichten ${ }^{114}$ aus dem gesamten Korpus stark hervorsticht.

Im Laufe des Dialogs \#2622 tauschen sich $A$ und $B$ über kuriose Vorfälle aus ihren Leben aus. Nachdem $A$ in der Nachricht davor erwähnt hat, dass ihm vor ein paar Monaten der Bildschirm seines iPhones kaputtgegangen ist, knüpft $B$ an das Thema an und berichtet über ihre schlechten Erfahrungen mit Handys und Smartphones. Es fällt dabei auf, dass Bin \#2 (wie auch im weiteren Verlauf des Dialogs) konsequent Auslassungspunkte benutzt, denen man eine segmentierende Funktion (vgl. Busch 2021: 395) zuschreiben kann.

114 Dialog \#2622 besteht aus 14 SMS-Nachrichten, wobei die kürzeste davon 70 Zeichen und die längste 684 Zeichen umfasst. Im Durchschnitt enthält eine Nachricht aus \#2622 aber 361 Zeichen, wohingegen dieser Wert für das gesamte russische Subkorpus bei 71 Zeichen pro Nachricht liegt. Die in (131) abgebildete Nachricht ist die viertlängste in diesem Dialog. 
(131) Auszug aus dem Dialog \#2622:

$\rightarrow$\begin{tabular}{c} 
Не заметила того сообщения...ну мало ли...))Знаешь, мне много кто \\
обещал вырвать все пирсинги... про телефоны можешь не говорить, \\
я с ними вообще не дружу...я их и в ванне купала, и ещё у меня два \\
раза за месяц ломался сенсорный телефон при чём оба раза \\
одинаково и оба раза хрен знает почему в магазине на меня \\
посмотрели как на идиотку,когда я пришла третий раз...другому \\
телефону я пыталась приделать панель и зубами экран..как бы это \\
сказать...разбила...я на спор натирала руки перцем...так круто \\
потом жжёт их весь день мммм))) \\
\hline $\begin{array}{c}\text { Iсh habe jene Nachricht nicht gesehen...na ja mag sein...))Weißt du, } \\
\text { viele haben mir versprochen, mir meine Piercings rauszureißen... von den } \\
\text { Handys brauchst du mir nicht zu erzählen, ich habe immer Probleme mit } \\
\text { denen...ich habe die schon in die Badewanne getaucht, und dazu kommt } \\
\text { noch, dass mein Smartphone zwei Mal in einem Monat kaputtgegangen } \\
\text { ist, und zwar gab es beide Male das gleiche Problem und beide Male, } \\
\text { weiß der Geier, woran es lag. Man hat mich im Laden wie so eine Idiotin } \\
\text { angeschaut, als ich zum dritten Mal hingegangen bin...an einem anderen } \\
\text { Smartphone wollte ich die rückseitige Abdeckung anbringen und habe } \\
\text { den Bildschirm mit den Zähnen...wie soll ich es sagen...zerschlagen...ich } \\
\text { habe auch meine Handflächen mit Pfeffer um die Wette gerieben...sie } \\
\text { brennen dann den ganzen Tag so cool mmhm))) }\end{array}$ \\
\hline Nachricht \#2 - 18.05.2011 - 22:44 \\
\hline
\end{tabular}

Der für die Analyse einer selbstinitiierten Selbstreparatur relevante Ausschnitt aus \#2 enthält vier Setzungen von Auslassungspunkten: „...другому телефону я пыталась приделать панель и зубами экран..как бы это сказать...разбила...“ (,...an einem anderen Smartphone wollte ich die rückseitige Abdeckung anbringen und habe den Bildschirm mit den Zähnen..wie soll ich es sagen...zerschlagen...'). Dabei trennen die erste und die letzte Setzung der Auslassungspunkte diesen semantisch und syntaktisch abgeschlossenen Abschnitt vom Rest der Nachricht ab, sodass sie in Anlehnung an Busch (2021: 401) als grammatisch-syntaktisch motiviert angesehen werden können. Die beiden abschnittsinternen Setzungen, die die Reparaturinitiierung „..как бы это сказать...“ (,..wie soll ich es sagen...') umklammern, haben die Funktion, eine bei einer prospektiven Reparatur in der medial mündlichen Kommunikation vorkommende Pause zu emulieren. Somit ist diese Verwendung von Auslassungspunkten nach Busch (2021: 398, 401) eher rhetorisch-stilistisch bedingt, da die- 
se als „phonographische Pausenzeichen“ (vgl. „typographic silence“ bei Ong 2011: 228 und „typographische Pause“ bei Moraldo 2012: 195) fungieren. Busch (2021: 401) merkt in diesem Zusammenhang an, dass Auslassungspunkte die lineare Struktur der Beiträge unterbrechen und somit eine Verzögerung erzeugen, sodass sich die „Zeitlichkeit in der Räumlichkeit des Geschriebenen“ niederschlägt, was in (131) der Fall ist.

Neben Klammern und Auslassungspunkten liegt in zwei Beispielen aus dem deutschen Subkorpus eine weitere graphische Ressource vor, die die durchgeführte Reparatur zusätzlich hervorhebt, nämlich die Majuskelschreibung. In den beiden Fällen (132) und (133) betrifft die Großschreibung die Negationspartikel nicht, die nachrichtenfinal erscheint und vom Rest der Nachricht mit Auslassungspunkten abgegrenzt wird. Dieser Gebrauch von nicht scheint auf die vergleichbare Verwendung von not im Englischen zurückzugehen, ${ }^{115}$ das Haiman (1995: 331) als „utterance deflater“ bezeichnet und mit „He’s a snappy dresser.....not!“ oder „Bush will kill that program. Not.“ veranschaulicht. ${ }^{116}$ In solchen Fällen wird eine sprachliche Handlung zurückgenommen, wodurch durch den gleichzeitigen Zugang zur ursprünglichen und zur negierten Version eine ironische oder sarkastische Wirkung erzeugt wird (vgl. das Durchstreichen bei Piperski/Somin 2014a, 2015b; vgl. 3.4.2.1). Simpson (2003: 139) beschreibt diese Wirkung folgenderweise:

It is through the technique of negation that the satirical ,mirror' is able to show the same conceptual domain, but only in reserve, and as a kind of negated doppel-gänger in discourse.

Bemerkenswert ist außerdem, dass notals utterance deflater (Haiman 1995: 331) inzwischen den Eingang in die Twitter-Kommunikation gefunden hat und in Form eines Hashtags gebraucht wird, um ebenso Ironie bzw. Sarkasmus zu markieren (vgl. „So I just colored with Ava for an hour. Yeah my summer so far has been so fun $(0)$ \#not" bei Sulis et al. 2016: 133).

Haiman (1995: 331) weist zwar darauf hin, dass der Rezipient eine sprachliche Handlung bis zum Auftreten der Negation als utterance deflater ernsthaft interpretieren könnte. In den beiden in den Daten gesichteten Fällen (132) und

115 In Bezug auf die Herkunft dieser Verwendung von not findet man in der Forschungsliteratur unterschiedliche Hypothesen: Als deren Ursprungsquelle wird die US-Comedy-Show Saturday Night Live (1978), der Film Wayne's World (1992) oder der Zeichentrickfilm Little Nemo (1910) genannt (vgl. Haiman 1995: 331, 1998: 54).

116 Vgl. auch den adversativen Konnektor ama in der äußerungsfinalen Position im Bulgarischen in der vergleichbaren Funktion bei Fielder (2010: 42). 
(133) scheint aber die ironische Interaktionsmodalität bereits vor nichtim unterschiedlichen Ausmaß aktiviert zu sein. Während in (132) der ironische Charakter des betroffenen Teils der Nachricht eher implizit aus dem Wissen des Rezipienten über die Konzerte der Gruppe abgeleitet werden kann, manifestiert sich die ironische Einstellung in (133) bereits zu Beginn der Nachricht durch den Gebrauch der Partikel boah:

(132) Auszug aus dem Dialog \#1306:

Schreiber $A$

Schreiber $B$

Moin Kollege, alles fitto? Wollt mal kurz hören, wie es bei dir nächstes we aussieht? Hast du noch ne Karte für (NAME DER BAND) bekommen? Beste Grüße

Nachricht \#1 - 08.11.2011 - 20:35:00

Ja moinsen, ne leider nicht. Wieso, hast du eine übrig?

Nachricht \#2 - 08.11.2011 - 20:38:05

$\rightarrow$\begin{tabular}{c}
$\begin{array}{c}\text { Nope, aber (VORNAME) wollte wissen, ob er für dich auch die hin-und } \\
\text { Rückfahrt einplanen soll. Schade, aber wird bestimmt eh total } \\
\text { langweilig....NICHT!!; }\end{array}$ \\
\hline Nachricht \#3 - 08.11.2011 - 20:40:05 \\
\hline
\end{tabular}

Die sind live ja auch nur wenig geil. Watt soll's, Euch viel Spaß!

Nachricht \#4 - 08.11.2011 - 20:45:00

In (132) wollten Schreiber $A$ und $B$ gemeinsam mit ihren Freunden zu einem Konzert einer Band gehen, $B$ konnte bisher allerdings keine Eintrittskarte bekommen. Daher scheint $B A$ ’s Vorschlag zunächst als eine Präsequenz zu einem Kartenangebot zu interpretieren. $A$ 's Frage ist aber durch die Planung für die Fahrt zum Konzert motiviert, was er in \#3 expliziert. Anschließend beginnt er eine Tröstungssequenz, in der er $B$ versichert, dass das Konzert „eh total langweilig“ (\#3) wird. Diese Tröstungssequenz stellt sich aber durch das Einfügen der nachrichtenfinalen Negationspartikel „NICHT!!“ (\#3) als eine Frotzelaktivität heraus. Dadurch, dass nicht erst am Ende der Nachricht geliefert wird, wird das Erkennen der vorliegenden Frotzelaktivität herausgezögert, wozu auch die iterierten Auslassungspunkte beitragen (zu verzögernden Auslassungspunkten vgl. Busch 2021: 398- 401). Die Majuskelschreibung dient der graphischen Hervorhebung von nicht, wobei sich in diesem Zusammenhang die Frage stellt, ob die Negationspartikel durch ihre somit erzeugte graphische Prominenz doch 
nicht früher wahrgenommen wird, da Lesen nicht strikt linear erfolgt und der Blick auf Salientes bevorzugt gelenkt wird.

Im Gegensatz zu (132) kann der erste Teil der Nachricht „Boah wie ich dieses Wetter hier vermisst habe“ aus (133) auch ohne Negation durch den Kontext, der im vorherigen Verlauf des Dialogs konstituiert wird, als ironisch verstanden werden. Die nachrichtenfinale Negationspartikel sollte daher vermutlich den ironischen Charakter von B's Stellungnahme zum aktuellen Wetterzustand vielmehr zusätzlich hervorheben bzw. kontextualisieren als im engeren Sinne offenlegen:

(133) Auszug aus dem Dialog \#3873:

Schreiberin $A$ Schreiberin $B$

\begin{tabular}{|c|}
\hline Besprechen wir, wenn wir alle heile angekommen sind \\
\hline Nachricht \#16 - 06.01.2016 - 17:48 \\
\hline
\end{tabular}

\begin{tabular}{|c|}
\hline Alles klar, das klingt nach einem Plan \\
\hline Nachricht \#17 - 06.01.2016 - 17:50 \\
\hline
\end{tabular}

Also wir sind gut angekommen. Es war gar nicht mehr so arg glatt, aber die Autos sahen krass aus. Alle voller Eis.

Nachricht \#18 - 06.01.2016 - 18:30

Ich bin jetzt auch gleich am Bahnhof. Der Zugführer hat eine

Durchsage gemacht, dass man wegen Glätte aufpassen und nicht ausrutschen soll.

Nachricht \#19-06.01.2016 - 18:35

Boah wie ich dieses Wetter hier vermisst habe... NICHT!

Nachricht \#20 - 06.01.2016 - 18:47

Braucht keine Sau

Nachricht \#21 - 06.01.2016 - 19:00

\section{Lexikalische Reparaturinitiierungen: Partikeln und Konjunktionen}

Im deutschen Subkorpus bilden Partikeln bei selbstinitiierten Selbstreparaturen im same turn die größte Gruppe unter Reparaturmarkern: Sie machen mit 43 Fällen 65\% aller Reparaturinitiierungen im same turn aus. Dabei ist der Diskursmarker also (32 Tokens; vgl. (125) und (126)), der v.a. lexiko-semantische und 
pragmatische Reparaturen einleitet, mit Abstand der häufigste Reparaturmarker innerhalb der Gruppe von lexikalischen Reparaturinitiierungen, während nee nur in drei (vgl. (127)) und die Fokuspartikel zumindest (vgl. Fußnote 112) in zwei Belegen vorliegt. Solche Partikeln wie ach, eh (vgl. (135)), eigentlich, pardon, sorry und ups (vgl. (136)) sind in je einem Beispiel vertreten. Im Gegensatz zu den deutschen Daten sind Partikeln im russischen Subkorpus lediglich in zwei von 14 Reparaturen im same turn zu finden, was ca. 18\% aller Reparaturmarker in Selbstreparaturen innerhalb derselben Nachricht entspricht. In beiden Fällen handelt es sich um die Partikel $H y$ (,na ja, nun, halt, äh‘; vgl. (128) und (134)). Ein solch homogenes Bild bei Partikeln als Reparaturmarker im Russischen mag auf den ersten Blick plausibel erscheinen, da unter den untersuchten same turn repairs im russischen Subkorpus keine Korrekturen, sondern ausschließlich Elaborierungen vorkommen. Daher liegt es nahe, dass die Partikel $H y$ (,na ja, nun, halt, äh'), die für eine Reparaturinitiierung im Rahmen einer Elaborierung geeignet ist, in den untersuchten Daten vertreten ist, während andere für die gesprochene Sprache von Tsesarskaya/Shestopalova (2017) belegte Partikeln wie z.B. ой(,ups'), тьфу (,pfui') oder блин (,Mist'), die eher für eine Korrektur typisch sind, dagegen ausbleiben. Allerdings bedarf es weiterer empirischer Untersuchungen, um die Frage der Verwendung der russischen Partikeln bei Reparaturen in der informellen interaktionalen Schriftlichkeit zu klären, da es dennoch zumindest theoretisch denkbar wäre, z.B. die Partikel даже (,sogar`) ebenfalls bei einer Elaborierung im same turn zu benutzen.

An dieser Stelle soll nun das zweite von insgesamt zwei Beispielen mit $H y$ (,na ja, nun, halt, äh`) zur Veranschaulichung herangezogen werden, das erste wurde bereits in (128) diskutiert:

(134) Auszug aus dem Dialog \#2622:

Schreiber $A$

А я разбил градусник когда нагревал его под краном...сразу врубил горячую-он и раскололся...прям на кухне тоже все бегали, суетились...а в школу я все равно потом не пошел =)) а я деньги ел...и зубочистки...ну как ел...случайно заглатывал =) просто кто то сказал моей знакомой что в партии пельменей должен быть один "счастливый" с монеткой...ну она блин и взяла монетку... в 5 рублей, а я как то так не особо прожевал и проглотил...она потом так весело по горлу потом шла...думал сдохну...а еще я ногой гантелю ловил...я ее подгидыва а потом как то не поймал...

а у меня ж инстинкт-надо ногу подставить-чтобы не разбилось...я и подставил...не думал что знаю столько слов...=) 


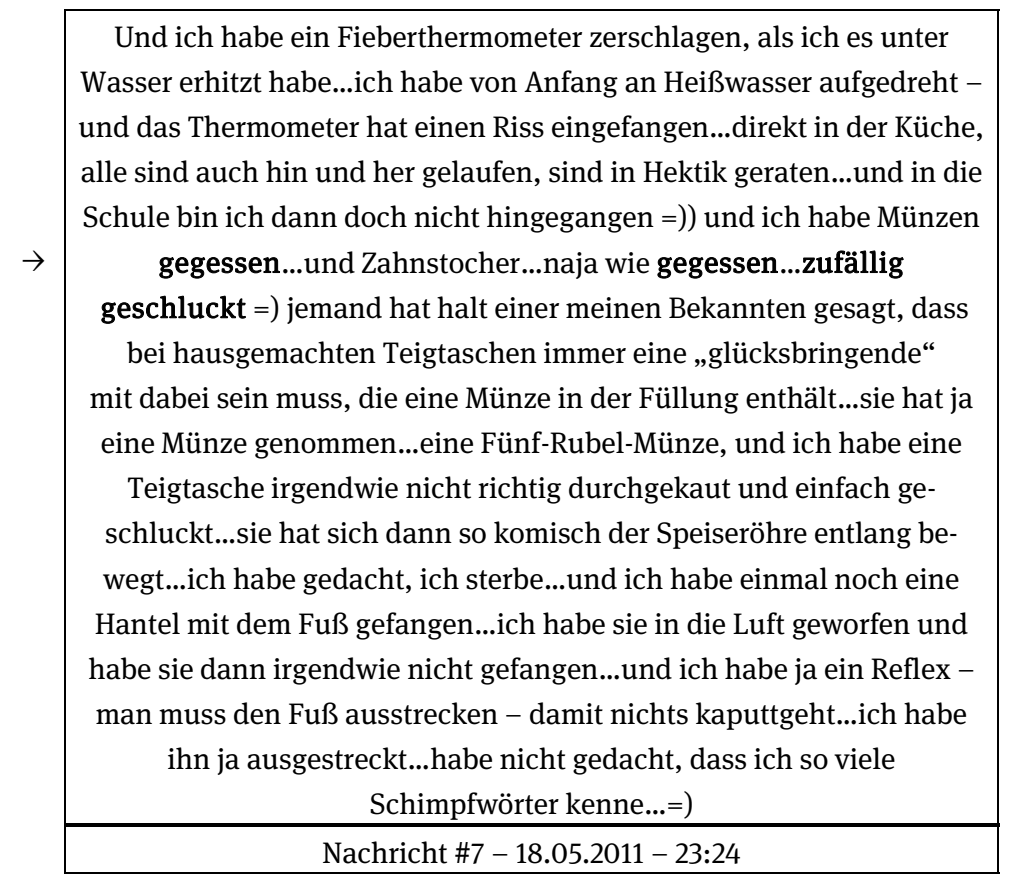

Nachricht \#7 aus (134) stammt aus dem gleichen Dialog wie (131), in dem Schreiber $A$ und Schreiberin $B$ komische Vorfälle aus ihren Leben miteinander teilen. In \#3-7 geht es hauptsächlich darum, welche ungewöhnlichen Sachen die beiden gegessen und getrunken haben. In diesem Kontext erwähnt Schreiber $A$ in \#7, dass er u.a. Münzen sowie Zahnstocher gegessen hat (,а я деньги ел...и зубочистки“ ,und ich habe Münzen gegessen...und Zahnstocher'), wobei Auslassungspunkte in dem Fall analog zu (131) eine rhetorisch-stilistisch bedingte Verzögerungsfunktion (vgl. Busch 2021: 398- 401) ausüben. Das als Reparandum fungierende Verb „ел“ (,essen-1P.SG.PRÄТ“) könnte dabei aber als ,mit Absicht verzehrt` interpretiert werden. Die potenziell beim Rezipienten durch das Verb „ел“ aktivierte semantische Komponente ,mit Absicht“ wird im Rahmen der anschließenden Elaborierung außer Kraft gesetzt. Dafür wird nach einer Reparaturinitiierung mit „ну как“ (,naja wie/naja, was heißt‘) die Problemquelle „ел“ (,essen-1P.SG.PRÄТ‘) wiederaufgenommen, sodass das Lokalisieren der Problemquelle für den Rezipienten erleichtert wird. Danach folgt die Insertion des Adverbs „случайно“ (,zufällig, versehentlich`) sowie die Substitution des Reparandums „ел“ (,essen-1P.SG.PRÄT“) durch ein anderes Verb „заглатывал“ (,schlucken-1P.SG.PRÄT‘), deren Kombination das Ausbleiben der Absicht beim Verzehren erwähnter Gegenstände zum Ausdruck bringen soll. 
Für die deutschen Daten wird zunächst eine mit der Partikel eh eingeleitete selbstinitiierte Selbstreparatur im same turn vorgestellt, die zwischen der lexiko-semantischen und pragmatischen Ebene anzusiedeln ist und eine sprachkreative Movierung einer Personenreferenz darstellt:

(135) Auszug aus dem WhatsApp-Dialog \#2904:

Schreiberin $A$

Schreiberin $B$

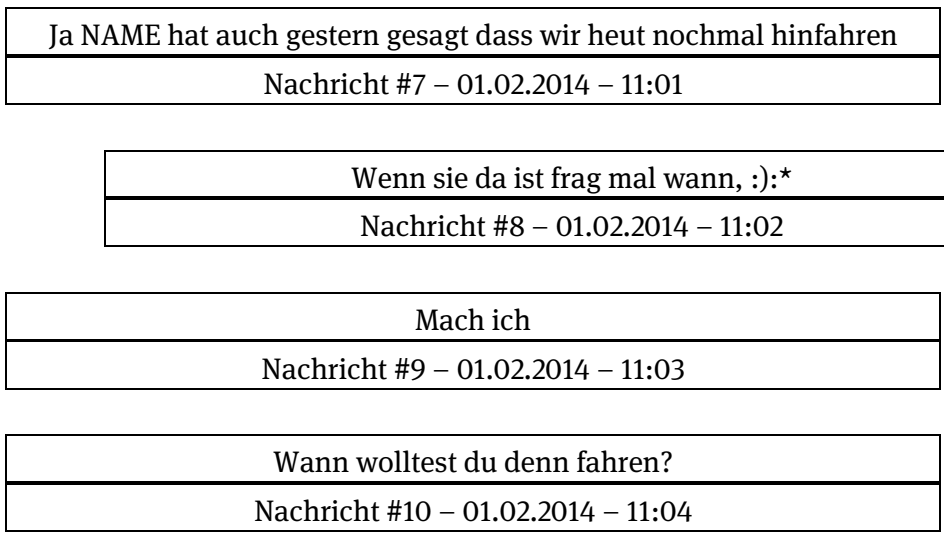

So zwischen 2und 3 damit ich nicht so spät n. Hause komme. :P Nachricht \#11 - 01.02.2014 - 11:05

\begin{tabular}{|c|}
\hline Und wollten sie lieber ganz allein dort sein? ;) :p \\
\hline Nachricht \#12-01.02.2014 - 11:05 \\
\hline
\end{tabular}

\begin{tabular}{|c|}
\hline Versteh ich nicht:) \\
\hline Nachricht \#13 - 01.02.2014 - 11:07 \\
\hline
\end{tabular}

Na ob du wissen willst, wann wir fahren um dann zu einer anderen Zeit zu gehen oder ob du mit uns fahren möchtest ;)

Nachricht \#14 - 01.02.2014 - 11:07

$\rightarrow$

Blödmann, eh frau. Damit wir uns dort treffen können. :P

Nachricht \#15 - 01.02.2014 - 11:09

\begin{tabular}{|c|}
\hline ;) :D \\
\hline Nachricht \#16 - 01.02.2014 - 11:09 \\
\hline
\end{tabular}




\begin{tabular}{|c|}
\hline hab ich mir gedacht :p \\
\hline Nachricht \#17 - 01.02.2014 - 11:09 \\
\hline
\end{tabular}

\begin{tabular}{|c|}
\hline Na also, geht doch! ;) :* \\
\hline Nachricht \#18 - 01.02.2014 - 11:10 \\
\hline
\end{tabular}

Schreiberinnen $A$ und $B$, die in einer Verwandtschaftsbeziehung zueinander stehen, stellen im Laufe des in (135) abgebildeten WhatsApp-Auszugs fest, dass sie am gleichen Tag dieselbe Person besuchen werden. Schreiberin $B$ hat $A$ bereits die ungefähre Zeit ihres geplanten Besuchs in \#11 mitgeteilt. Die scherzhaft gemeinte Frage in \#12 löst bei $B$ ein Verstehensproblem aus, sodass $A$ in \#14 die Motivation ihrer zurückliegenden Frage erläutert. Darauf reagiert $B$ mit dem Schimpfwort „Blödmann“, welches sie einer Movierung unterzieht. Dabei reinterpretiert $B$ das Lexem „Blödmann“, welches sich auf beide Geschlechter beziehen kann, und betrachtet dieses als ein produktives Kompositum, in dem die zweite Konstituente -mann durch das geschlechtsspezifische -frau ersetzt werden kann. Die Substitution wird mit der Partikel „eh“ angekündigt, wonach das Reparans "frau“ kommt, dessen Kleinschreibung als ein impliziter Hinweis dafür gedeutet werden könnte, dass dieses nicht das ganze Wort „Blödmann“, sondern nur eine seiner Konstituenten ersetzen soll. Diese Hypothese lässt sich dadurch stützen, dass $B$ im gesamten Dialog sowohl auf satzinitiale als auch -mediale Majuskelschreibung konsequent achtet.

Des Weiteren soll noch auf die Reparaturinitiierung mithilfe der Interjektion ups eingegangen werden, die im Rahmen der vorliegenden Arbeit auch für selbstinitiierte Selbstreparaturen im next opportunity space bereits beschrieben wurde (vgl. (121)):

(136) Auszug aus dem WhatsApp-Dialog \#1204:

Schreiber $A$

Schreiberin $B$

\begin{tabular}{|c|}
\hline Wie funktioniert das denn alles hier? \\
\hline Nachricht \#1 - 31.10.2011 - 14:37:05 \\
\hline
\end{tabular}

$\rightarrow$

Och nöhö,noch ne nervensäge mehr hier in der Liste?! Ups... HAllo

Tim, wie schön,du scheinst ein neues Handy zu haben ;)

Nachricht \#2 - 31.10.2011 - 15:14:05

Ja das hab ich. Aber dir schreib ich nicht mehr

Nachricht \#3 - 31.10.2011 - 15:17:05 


\begin{tabular}{|c|}
\hline Doooch \\
\hline Nachricht \#4 - 31.10.2011 - 15:21:05 \\
\hline
\end{tabular}

\begin{tabular}{|c|}
\hline Oder hast du deinen Humor heute im bett gelassen? \\
\hline Nachricht \#5 - 31.10.2011 - 15:21:10 \\
\hline
\end{tabular}

Nachrichten \#1 und \#2 deuten darauf hin, dass Schreiber $A$ offensichtlich ein neues Smartphone und damit erstmalig WhatsApp hat. Auf diese implizite Mitteilung reagiert Schreiberin $B$ zunächst mit einer scherzhaften Anmerkung „Och nöhö,noch ne nervensäge mehr hier in der Liste?!“ (\#2) und wechselt innerhalb derselben Nachricht in eine ernsthafte Interaktionsmodalität (vgl. Imo 2017), wobei die ,Übergangsstelle“ mit der Interjektion „Ups...“ markiert wird. Der somit von $B$ vollzogenen Frotzelaktivität (vgl. Günthner 1996, 1999) folgt jedoch ein „po-faced receipt“ (Drew 1987), sodass B face work betreiben und die Frotzelaktivität rechtfertigen muss.

Im Gegensatz zu Partikeln treten alternative Konjunktionen bzw. (vgl. \#4182), oder und или (,oder`) im Kontext von selbstinitiierten Selbstreparaturen im same turn seltener auf. Dabei handelt es sich um sechs lexiko-semantische Elaborierungen im deutschen und eine im russischen Subkorpus.

\section{Phrasale und satzwertige Reparaturinitiierungen}

Im deutschen und russischen Subkorpus liegen jeweils vier und fünf phrasale und satzwertige Reparaturmarker vor. Für das Russische sind dabei z.B. в смысле (,im Sinne von, ich meine‘; vgl. (140)), точнее (,eher/genauer gesagt‘; vgl. (124)) sowie как бы это сказать (,wie soll ich es sagen“; vgl. (131)) zu nennen. In den deutschen Kurznachrichten werden selbstinitiierte Selbstreparaturen v.a. mit Reparaturinitiierungen mit dem Verb meinen (vgl. (137) und (138)) eingeleitet. Während bei Reparaturen im next opportunity space das Verb meinen im Präsens und Präteritum Aktiv vorkommt, veranschaulichen die nächsten zwei Beispiele dessen Gebrauch bei selbstinitiierten Selbstreparaturen im same turn im Präsens Zustandspassiv:

(137) SMS-Dialog \#1880:

Schreiberin $A$

Schreiberin $B$

hi also nur zur info ich habe heute abend noch nichts vor ;-) wenn du zeit hast kannste ja bescheid geben. $\lg$ [spitzname]

Nachricht \#1 - 06.01.2012 - 18:38:20 
Also zur info, wir werden heute die stumpfe nummer machen. Erst bullenkopp, dann wilde hilde. Damit sind [name1], [name2] und ich gemeint. Um 10 ist meet and greet. What about [spitzname]? Biste dabei?

Nachricht \#2 - 06.01.2012 - 18:51:31

ach mist ich schaff es wohl doch nicht habe meinen text noch nicht fertig. 0 uhr deadline. dabei wäre ich nicht abgeneigt. hab gerad dem typen geschrieben.

Nachricht \#3-06.01.2012 - 21:16:44

In (137) zeigt Schreiberin $A$ ihre Bereitschaft, mit Schreiberin $B$ etwas an dem Abend gemeinsam zu unternehmen. Daraufhin schildert Schreiberin $B$, dass sie an dem Abend eine Kneipentour mit ein paar anderen Freunden geplant hat. Ein paar Stunden später teilt $A$ ihrer Freundin mit, dass sie doch viel zu tun hat und es nicht schaffen wird, sich der Kneipentour anzuschließen. Die hier im Fokus stehende selbstinitiierte Selbstreparatur liegt in \#2 vor und bearbeitet das Personalpronomen „wir“, das Schreiberin $B$ expliziert, indem sie die damit gemeinten Personen „[name1], [name2] und ich“ auflistet. Das Präpositionaladverb „Damit“ soll zwar auf das Reparandum verweisen, die Problemquelle ist jedoch eher durch die semantische Übereinstimmung zwischen dem Reparandum und dem Reparans erkennbar.

Die nächste Reparaturinitiierung mit meinen kommt in (138) vor und wird mit einem expliziten Metakommentar „Anmerkung“ (\#4) angekündigt:

(138) Auszug aus dem WhatsApp-Dialog \#4068:

Schreiberin $A$

Schreiberin $B$

VORNAME ich kann diese blöde Hausarbeit nicht schreiben :(( komme nicht klar!

Nachricht \#1 - 22.03.2016 - 17:20

Wieso?

Nachricht \#2 - 22.03.2016-17:22

Ich weiß gar nicht wie ich anfangen soll. Was soll man denn in der Einleitung großartig schreiben?

Nachricht \#3 - 22.03.2016 - 17:31 


\begin{tabular}{|c|c|}
\hline $\begin{array}{r}\text { Ich komme glaube ich etwas putzig herüber, aber so meine ich das } \\
\text { nicht } \text { Anmerkung: gemeint ist "patzig" }\end{array}$ \\
\hline Nachricht \#4-22.03.2016-17:36
\end{tabular}

Nein ach Quatsch, schick mir das mal wenn's fertig ist Packst du schon :*

Nachricht \#5 - 22.03.2016 - 17:36

Schreiberin $B$ beschwert sich in \#1 und \#3 bei $A$ über ihre Probleme mit der Hausarbeit. Anschließend reflektiert sie in \#4 ihr Verhalten und bewertet dieses als „putzig“, was sich im späteren Verlauf der Nachricht als Problemquelle herausstellt. Obwohl $B$ eigentlich die Möglichkeit hatte, das Reparandum mühelos im Rahmen einer Revision zu korrigieren, indem sie lediglich einen Buchstaben im Wort „putzig“ vor dem Abschicken der Nachricht ersetzen müsste, entscheidet sie sich für eine für die Rezipientin sichtbare Bearbeitung des Reparandums, die im Vergleich mit einer Revision aufwändiger ist. Es kann allerdings lediglich darüber spekuliert werden, aus welchem Grund Schreiberin $B$ die Reparatur einer Revision vorgezogen hat. Eine mögliche Erklärung könnte darin liegen, dass $B$ durch das gleichzeitige Auftreten des Reparandums und des Reparans eine für sie gesichtsbedrohende Bewertung etwas abschwächen bzw. die Interaktion etwas auflockern wollte. Abgesehen von B's Motivation liefert sie in \#4 das Reparandum „"patzig"“, das zum einen durch den vorangehenden Metakommentar „Anmerkung“ an den Teil der Nachricht mit der Problemquelle anknüpft und zum anderen mit der Reparaturinitiierung im Format gemeint ist $X$, wo $X$ für das Reparans steht, eingeleitet wird. Die bei Mostovaia (2018: 55) beschriebene Funktion von Anführungszeichen, bei fremdinitiierten Selbstreparaturen das wiederaufgenommene Reparandum zu markieren, kann somit durch eine weitere Funktion im Kontext von selbstinitiierten Selbstreparaturen ergänzt werden, nämlich das Reparans kenntlich zu machen. Die Verwendung von Anführungszeichen könnte in dem Fall evtl. mit dem Bestreben zu tun haben, das Reparans als metasprachlich darzustellen.

\subsubsection{Reparaturdurchführungen}

Reparaturdurchführungen bei Reparaturen in same turn weisen viele Gemeinsamkeiten mit Reparaturen im next opportunity space auf. Schreiber greifen hauptsächlich auf Substitutionen und Insertionen zurück (je fünf Substitutionen und Insertion in den russischen Daten, 19 Substitutionen und 24 Insertionen in den deutschen Kurznachrichtendialogen). Allerdings zeichnen sich same turn repairs im Gegensatz zu selbstinitiierten Selbstreparaturen im next und 
after next opportunity space dadurch aus, dass sie den Schreibern auch die Möglichkeit anbieten, neben retrospektiven Reparaturen, die eine zurückliegende Problemquelle bearbeiten, eine prospektive Reparatur, in deren Rahmen z.B. eine Wortsuche erfolgt (vgl. (131)), zu inszenieren (für das Deutsche vgl. \#3815 in der MoCoDa).

\section{Substitutionen}

Im Rahmen von im same turn durchgeführten Substitutionen werden in den meisten Fällen einzelne Lexeme durch andere Lexeme ersetzt (vgl. (124) und (134)). Bei der in (139) vorliegenden Substitution wird ein Schimpfwort einer Movierung unterzogen:

(139) Auszug aus dem WhatsApp-Dialog \#4216:

Schreiber $A$

Schreiberin $B$

\begin{tabular}{|c|}
\hline Du glaubst nicht, was mir passiert ist \\
\hline Nachricht \#1 - 25.08.2016 - 18:05:05 \\
\hline
\end{tabular}

\begin{tabular}{|c|}
\hline Ich hab ne Vermutung $\wedge^{\wedge}$ \\
\hline Nachricht $\# 2-25.08 .2016-18: 20: 05$ \\
\hline
\end{tabular}

Ich komm grad einfach nicht klar und weiß nicht, was ich tun soll

Nachricht \#3 - 25.08.2016 - 18:25:05

lass mich raten, das date neulich lief nicht so wie geplant? :D

Nachricht \#4 - 25.08.2016 - 18:26:05

$\rightarrow$

Nein...Ja....du Arsch! Pardon, als Repräsentatnten der Münsteraner

Hochschulpolitik wollen wir politisch korrekt sein: Du Arsch^in bzw.

Ärschin!

Nachricht \#5 - 25.08.2016 - 18:26:05

\begin{tabular}{|c|}
\hline \#Genderwahn Dann erzähl! :D \\
\hline Nachricht \#6 - 25.08.2016 - 18:27:05 \\
\hline
\end{tabular}

Nach einem „story preface“ (vgl. Jefferson 1978; Stivers 2013: 200-203) in \#1, das in \#3 weiter ausgebaut wird, kündigt Schreiberin $B$ eine Vermutung über den Gegenstand der Erzählung an, welche sie anschließend in \#4 äußert. Die von $B$ in \#4 aufgeführte Hypothese wird allerdings von $A$ in Nachricht \#5 zuerst zurückgewiesen, dann aber doch bestätigt, woraufhin eine beleidigende Fremd- 
zuschreibung „du Arsch!“ folgt. Diese Fremdzuschreibung dient aber gleichzeitig als Ausgangspunkt für eine selbstinitiierte Selbstreparatur, in deren Rahmen Schreiber $A$ nach dem repair preface (vgl. Kitzinger 2013: 240-241) „Pardon“ und einer Argumentation für die anschließend vorgenommene Movierung versucht, vom Nomen Arsch eine entsprechende Bezeichnung für eine weibliche Person abzuleiten. Dabei bietet $A$ zwei Formen an: Das erste kreative Wortbildungsprodukt „Arsch`in“ wird mithilfe des Gender-Sternchens gebildet, während das alternative Derivat „Ärschin“ an die Movierung wie Arzt-Ärztin angelehnt zu sein scheint. $B$ reagiert auf diese okkasionellen Wortschöpfungen mit einem Kommentar „\#Genderwahn“ und fordert $A$ auf, ihr die Geschichte zu erzählen. Diese, wie die Fortsetzung des Dialogs zeigt, hat nichts mit der davor erwähnten Verabredung von $A$ zu tun, sondern bezieht sich auf eine Flasche mit selbstgemachtem Kürbissirup, die in $A$ 's Kühlschrank explodiert ist und die Regale beschädigt hat.

Neben einzelnen Wörtern bzw. in einigen Fällen Phrasen liegt im deutschen Subkorpus eine Substitution vor, in der ein Morphem ersetzt wird (vgl. (135)). Des Weiteren könnte man die in (127) beschriebene pragmatische Korrektur ebenso als eine Substitution betrachten, in der eine scherzhafte Antwort „Hä, ich red doch nicht mit jedem“ zurückgenommen und durch eine ernsthafte „wollte gleich zurück rufen. Echt“ abgelöst wird (vgl. auch (136)).

\section{Insertionen}

In den analysierten Reparaturen im same turn aus dem deutschen Subkorpus kommen Insertionen ebenso wie bei Reparaturen im next opportunity space etwas seltener als Substitutionen vor, während die russischen selbstinitiierten Selbstreparaturen im same turn die gleiche Anzahl von Substitutionen und Insertionen aufweisen (zu Insertionen in den deutschen Daten vgl. (129), (132) und (133)). Zur Veranschaulichung wird hier ein Beispiel aus dem russischen Korpus herangezogen:

(140) iMessage-Dialog \#3043:

Schreiberin $A$

Schreiberin $B$

$\rightarrow$\begin{tabular}{c|} 
привет!:) как дела? [женское имя], я хотела спросить дискотека \\
во сколько будет на слете? и что брать с собой? именно на \\
дискотеку в смысле)
\end{tabular} \mid \begin{tabular}{c} 
Hallo!:) Wie geht es dir? [Frauenname], ich wollte fragen, wann die \\
Disko beim Jugendforum stattfindet? Und was soll man mitbringen? \\
Gerade für die Disko meine ich) \\
\hline Nachricht \#1 - 24.10.2013-15:38:00
\end{tabular}


Привет, милая;))^ Все хорошо, как у тебя дела? Дискотеки не будет. Открытие будет в стиле диско. Одеваемся, как договорились)) )

Hallo meine Liebe;)^^ Bei mir ist alles gut, wie geht es dir? Es wird keine Disko geben. Die Forumseröffnung findet im Disco-Style statt.

Wir ziehen das an, was wir vorher abgesprochen haben)) )

Nachricht \#2 - 24.10.2013 - 15:46:00

\begin{tabular}{|c|}
\hline aa,хорошо) ) \\
\hline Achso, geht klar) ) \\
\hline Nachricht \#3 - 24.10.2013 - 16:02:00 \\
\hline
\end{tabular}

Die in (140) vorliegende selbstinitiierte Selbstreparatur ist einem der wenigen iMessage-Dialoge im Korpus entnommen. Dabei erkundigt sich Schreiberin $A$ bei ihrer Freundin darüber, was sie mitnehmen soll, nennt aber vorher zwei Veranstaltungen слёт (,Jugendforum') und дискотека (,Diskothek'), sodass unklar bleibt, auf welche der beiden sich $A$ 's Frage bezieht. In der darauffolgenden selbstinitiierten Selbstreparatur wird durch die Insertion der Präpositionalphrase „на дискотеку“ (,für die Disko') der Bezug der Frage erfolgreich disambiguiert. Das lässt sich an B's Reaktion in \#2 erkennen, die Schreiberin $A$ erklärt, dass es eigentlich keine Diskothek, sondern lediglich eine Forumseröffnung im Disco-Style geplant ist. Außerdem erinnert $B$ ihre Kommunikationspartnerin an eine zuvor getroffene Verabredung in Bezug auf den Bekleidungsstil.

\subsubsection{Reaktionen auf selbstinitiierte Selbstreparaturen im same turn}

Bei Reparaturen im same turn finden sich relativ wenige Fälle (drei im russischen und acht im deutschen Korpus), bei denen eine Reaktion des Rezipienten der Problemquelle auf eine Reparaturdurchführung erfolgt. Dabei kann die in der Reparatur getroffene Aussage bestätigt (vgl. (141)), zurückgewiesen (vgl. (136)) oder kommentiert werden (vgl. (142)):

(141) Auszug aus dem Dialog \#2383:

Schreiberin $A$

Schreiberin $B$

als VORNAME kann nicht ansonsten kommen alle. aber VORNAME will unebdingt ihren freund mitbringen....:( ich will den nicht dabei haben

Nachricht \#4 - 01.11.2013 - 18:34:05

och ne wieso das denn? :-o mädelsabend heißt ohne jungs...

Nachricht \#5 - 01.11.2013 - 19:04:05 


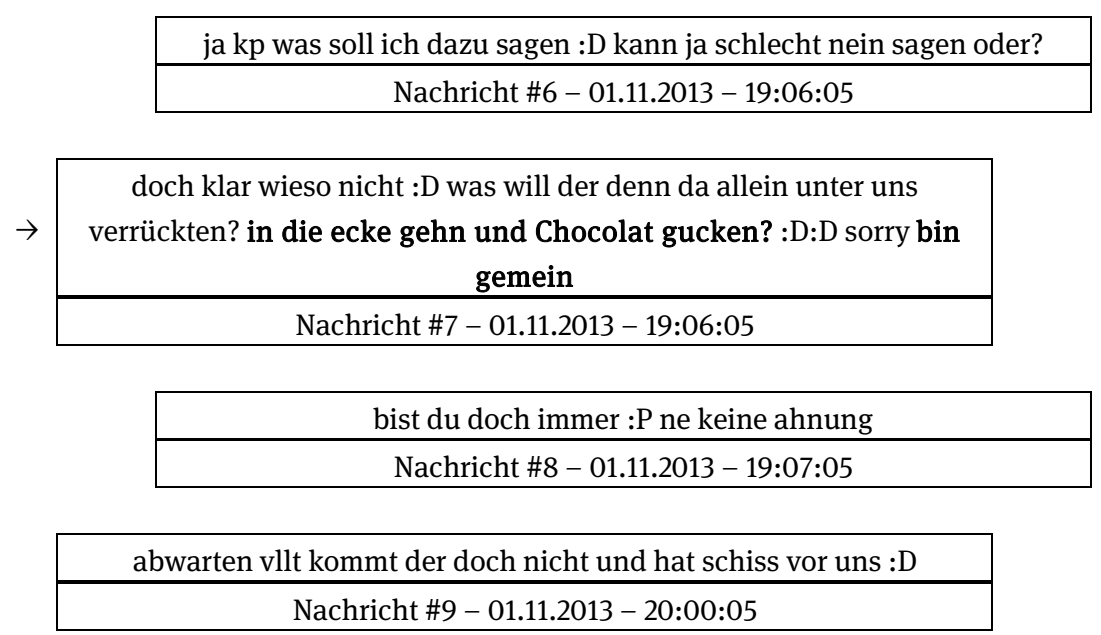

In (141) beschwert sich Schreiberin $B$ bei ihrer Freundin darüber, dass eine weitere Freundin von den beiden ihren Freund zum dem geplanten ,Mädelsabend mitbringen möchte. Darauf reagiert $A$ in \#7 mit einer sarkastischen Bemerkung darüber, womit sich dieser Freund während des Abends beschäftigen könnte. Direkt im Anschluss schwächt $A$ allerdings ihre gesichtsbedrohende Äußerung wieder ab, indem sie die negative Selbstbewertung „sorry bin gemein“ (\#7) abgibt. $B$ greift diese Reparaturdurchführung von $A$ auf und bestätigt diese negative Charakterisierung von $A$ im Rahmen einer Frotzelaktivität in \#8, welche sie durch das Emoticon „:P“ kenntlich macht.

Eine Kommentarfunktion wird bei den untersuchten same turn repairs von der bereits unter (139) diskutierten Antwort „\#Genderwahn“ sowie Emoticons, die in drei weiteren Dialogen festgestellt wurden (vgl. Pappert 2017: 196-198), übernommen. Einer davon liegt in (142) vor:

(142) Auszug aus dem Dialog \#2356:

Schreiberin $A$

Schreiberin $B$

Hab grad mein erstes Weihnachtsgeschenk gekauft <3 (Bilddatei von

Ledertasche angehängt)

Nachricht \#6 - 30.10.2013 - 14:35

Für wen? :o Geil <3

Nachricht \#7 - 30.10.2013 - 14:44

Ja tut mir leid :/ Hätte sonst gerne was mit dir gemacht :*

Nachricht \#8 - 30.10.2013-14:44 


\begin{tabular}{|c|}
\hline Ja für mich :)) Ist doch kein problem <3 \\
\hline Nachricht $\# 9-30.10 .2013-14: 52$ \\
\hline
\end{tabular}

\begin{tabular}{|c|}
\hline Geiles Teil $<3$ \\
\hline Nachricht \#10 - 30.10.2013 - 15:06 \\
\hline Hrr Danke ;) Ach du meinst die Tasche.. ja.. ok :( :D \\
\hline Nachricht \#11 - 30.10.2013 - 15:11 \\
\hline :D :D :D :D du sowieso ;) ;) \\
\hline Nachricht \#12 - 30.10.2013 - 15:11 \\
\hline$: \mathrm{D}: \mathrm{D}::^{\star}{ }^{\star}$ \\
\hline Nachricht \#13-30.10.2013-15:15 \\
\hline$:^{\star}:^{\star}:^{\star}$ \\
\hline Nachricht \#14 - 30.10.2013 - 15:16 \\
\hline
\end{tabular}

In \#6 teilt Schreiberin $A$ ihrer Freundin mit, dass sie sich eine neue Ledertasche als Weihnachtsgeschenk gekauft hat, und schickt ihr ein Foto davon. Nach einer positiven Bewertung der Tasche durch $B(\# 10)$ bedankt sich $A$, stellt aber anschließend fest, dass sich $B$ 's Bewertung auf die Tasche und nicht auf sie selbst bezieht, da sie vermutlich ebenso auf dem verschickten Foto zu sehen war. $B$ reagiert auf $A$ 's Reparatur mit iterierten ,weit lächelnden' Emoticons und versichert ihr, dass die positive Bewertung auch auf $A$ selbst zutreffen würde.

\subsubsection{Interaktionale Funktionen von selbstinitiierten Selbstreparaturen im same turn}

Während selbstinitiierte Selbstreparaturen im next und after next opportunity space eine Problemquelle bearbeiten, die sich in einer bereits verschickten Nachricht befindet, richten sich selbstinitiierte Selbstreparaturen im same turn auf Reparanda, die in der emergenten, noch nicht abgeschickten Nachricht liegen. Dabei bietet es sich zwar an, eine Problemquelle im Rahmen einer Revision zu bearbeiten und somit diese für den Rezipienten unsichtbar zu lassen, jedoch ziehen es Schreiber in 14 Fällen im russischen und 60 im deutschen Subkorpus vor, stattdessen eine für den Rezipienten zugängliche selbstinitiierte Selbstreparatur durchzuführen. Eine solche Entscheidung scheint v.a. dadurch begründet zu sein, dass das gleichzeitige Vorkommen des Reparandums und des Reparans als eine besondere rhetorisch-stilistische Ressource benutzt wird, 
um beispielsweise eine hyperbolische Äußerung abzuschwächen (vgl. (128) sowie Fußnote 112). Außerdem werden Reparaturen im same turn analog zu Reparaturen im next opportunity space bei scherzhaft-ironischen Aktivitäten eingesetzt, beispielsweise bei Frotzelaktivitäten, wie „Schade, aber wird bestimmt eh total langweilig....NICHT!!;)“ aus (132). Dabei scheint der Gebrauch des nachrichtenfinalen nicht nur auf Reparaturen im same turn beschränkt zu sein. Schließlich kann mithilfe von verbalen sowie graphischen Verzögerungssignalen (vgl. Busch 2021: 398-401) im same turn eine prospektive Reparatur inszeniert werden, um eine für die gesprochene Sprache typische zeitliche Entfaltung der Gedanken zu simulieren und somit eine besondere stilistische Wirkung der Nachricht zu erlangen. Das trifft v.a. auf die Reparatur aus Dialog \#2622 zu (vgl. „другому телефону я пыталась приделать панель и зубами экран..как бы это сказать...разбила...“ ,an einem anderen Smartphone wollte ich die rückseitige Abdeckung anbringen und habe den Bildschirm mit den Zähnen..wie soll ich es sagen...zerschlagen...' aus (131)).

\subsubsection{Selbstinitiierte Selbstreparaturen im after next opportunity space}

Selbstinitiierte Selbstreparaturen im after next opportunity space sind in den russischen und den deutschen Daten jeweils mit drei und sieben Belegen vertreten, was je 6\% und 3\% der gesamten Anzahl von selbstinitiierten Selbstreparaturen (ohne third position repair) im entsprechenden Subkorpus ausmacht. Somit kommen selbstinitiierte Selbstreparaturen im after next opportunity space in den analysierten Daten deutlich seltener als bereits beschriebene selbstinitiierte Selbstreparaturen im next opportunity space (je 69\% und 67\% im russischen und deutschen Korpus) sowie im same turn (je 25\% und 29\%) vor. Diese quantitative Verteilung zeigt also, dass Produzenten der Problemquelle einen großen Wert auf die schnelle Bearbeitung des zurückliegenden Reparandums (für die gesprochene Sprache vgl. Schegloff 1979, 2007; Heritage 2007; Pfeiffer 2017a) legen, damit die Problemquelle sowie die Reparaturdurchführung möglichst dicht aneinander platziert werden. Das kann am besten erreicht werden, wenn die Reparaturdurchführung entweder in der gleichen Nachricht erfolgt, in der sich auch die Problemquelle befindet, oder in der direkt darauffolgenden. Je später das Reparandum bearbeitet wird, desto schwieriger ist es für den Rezipienten, dieses zu lokalisieren und mit der gelieferten Reparaturdurchführung in Beziehung zu setzen bzw. sogar eine Reparaturdurchführung als solche zu erkennen. Das betrifft v.a. diejenigen Fälle, bei denen eine Repara- 
turinitiierung mit expliziten Reparaturmarkern ausbleibt und/oder lediglich das Reparans ohne syntaktischen Anker als Reparaturdurchführung geliefert wird.

In den untersuchten Beispielen ist die Verschiebung der Reparatur aus dem next opportunity in den after next opportunity space durch zwei Gründe bedingt. Zum einen kommt sie dadurch zustande, dass die Nachricht mit der Problemquelle in zwei Nachrichten aufgesplittet wird (vgl. Beißwenger 2007), sodass der next opportunity space, in dem die Reparatur potenziell durchgeführt werden könnte, bereits durch die Fortsetzung der problematischen Nachricht besetzt ist (vgl. (143) und (144)). Zum anderen kann zwischen der Nachricht mit der Problemquelle und der Nachricht mit der Reparaturdurchführung ein Beitrag liegen, der eine ganz andere sprachliche Handlung enthält (vgl. (145)). Im letzten Fall kann jedoch lediglich spekuliert werden, ob eine weitere sprachliche Handlung in einem konkreten Kontext der Reparaturdurchführung vorgezogen wurde, weil der Produzent der Problemquelle diese Handlung als eine interaktional relevantere einstuft und diese daher früher vollzieht oder weil er die Notwendigkeit der Reparatur erst später erkennt.

Wegen der geringen Anzahl an Belegen wird dieses Kapitel nicht wie 6.1.1 und 6.1.2 untergliedert, sondern die im Korpus vorgefundenen Reparandatypen, Reparaturmarker sowie Reparaturoperationen werden im Folgenden exemplarisch anhand von vier ausgewählten Beispielen parallel zueinander veranschaulicht. Im deutschen Teilkorpus werden ein (ortho)graphisches sowie drei pragmatische Reparanda (vgl. (146)) im after next opportunity space bearbeitet. Zwei weitere Problemquellen aus den deutschen Daten lassen sich mehr als einem Reparandatyp zuordnen: So liegt in (122a-b) ein Reparandum vor, das gleichzeitig als (ortho)graphisch und lexiko-semantisch anzusehen ist (vgl. \#1 und \#4), während die Problemquelle aus (144) sowohl als (ortho)graphisch als auch als morphosyntaktisch eingestuft werden kann. Im russischen Subkorpus kommen ein (ortho)graphisches (vgl. (143)) und zwei lexiko-semantische Reparanda (vgl. (145)) vor.

Die häufigste Reparaturoperation, auf die sowohl in den russischen als auch in den deutschen Reparatursequenzen zurückgegriffen wird, stellt die Substitution dar (vgl. (143)-(145)). Des Weiteren findet sich in den deutschen Daten eine Insertion, bei der ein Fragezeichen nachgereicht wird. Wenn man annimmt, dass die an einen Mann gerichtete Anrede „süsse“ (\#9) in der pragmatischen Reparatur aus (146) zurückgenommen wird, könnte man diese als Tilgung im weiteren Sinne auffassen (für ein weiteres Beispiel vgl. \#2379).

In Hinblick auf die Reparaturinitiierung weisen drei deutsche und zwei russische selbstinitiierte Selbstreparaturen im after next opportunity space explizite Reparaturmarker auf. Dabei handelt es sich für das Deutsche in erster Linie 
um Partikeln ach, ai (vgl. (146)), nicht (vgl. (144)) und für das Russische um einen Asterisk (vgl. (145)) sowie um die nicht-standardsprachliche Abkürzung eines phrasalen Reparaturmarkers в смысле (,im Sinne von, das heißt'), nämlich $B C M$, in der die Präposition $B$ (,in') mit den ersten zwei Buchstaben des Nomens смысле (,Sinn-PREP‘) verschmolzen ist (vgl. \#4330).

Das erste in diesem Kapitel diskutierte Beispiel (143) stammt aus einem Dialog, der damit beginnt, dass Schreiberin $A$ ihrem Interaktionspartner mitteilt, dass sie die WhatsApp-Software auf ihrem Smartphone erfolgreich installiert hat. Danach begrüßt Schreiber $B$ sie als neue WhatsApp-Nutzerin und äußert seine positive Meinung zu WhatsApp als Messenger-Dienst:

(143) Auszug aus dem WhatsApp-Dialog \#3217:

Schreiberin $A$

Schreiber $B$

\begin{tabular}{|c|}
\hline Ну вот а после года плати \\
\hline Ja und dann muss man nach einem Jahr bezahlen \\
\hline Nachricht \#25 - 24.03.2014 - 14:05:05 ${ }^{117}$ \\
\hline
\end{tabular}

$\rightarrow$

\begin{tabular}{|c|}
\hline И смысл тогда... чл смс смог ватсапп оплачивать... \\
\hline $\begin{array}{c}\text { Und welchen Sinn hat das denn... sowohl SMS konnte WhatsApp muss } \\
\text { man bezahlen... }\end{array}$ \\
\hline Nachricht \#26 - 24.03.2014 - 14:05:05 \\
\hline
\end{tabular}

\begin{tabular}{|c|}
\hline Ежемесячно \\
\hline Monatlich \\
\hline Nachricht \#27-24.03.2014-14:05:05 \\
\hline
\end{tabular}

$\rightarrow$

\begin{tabular}{|c|}
\hline Что смс что ватсапп: \\
\hline Sowohl SMS als auch WhatsApp: \\
\hline Nachricht \#28 - 24.03.2014 - 14:05:05 \\
\hline
\end{tabular}

\begin{tabular}{|c|}
\hline Ксюх, подписка на год стоит доллар \\
\hline Ksjuscha, die Gebühr beträgt einen Dollar pro Jahr \\
\hline Nachricht \#29 - 24.03.2014 - 14:05:05 \\
\hline
\end{tabular}

117 Bei den Zeitangaben in diesem WhatsApp-Dialog handelt es sich um die von der MoCoDa automatisch zugewiesenen Zeitstempel, die von der Datenbank für solche Nachrichten generiert werden, für die keine Informationen über den Zeitpunkt des Abschickens oder des Empfangs der Nachrichten beim Spenden der Daten eingetragen wurden. 


\begin{tabular}{|c|}
\hline А не в месяц \\
\hline Und nicht pro Monat \\
\hline Nachricht \#30 - 24.03.2014 - 14:05:05 \\
\hline
\end{tabular}

\begin{tabular}{|c|}
\hline Aaaa \\
\hline Achsooo \\
\hline Nachricht \#31 $-24.03 .2014-14: 05: 05$ \\
\hline
\end{tabular}

Nach einer längeren Einschubsequenz, in der die beiden über eine gemeinsame Freundin gelästert haben (nicht abgebildet), wird der WhatsApp-Messenger wieder zum Thema. Schreiberin $A$ nimmt im Gegensatz zu $B$, der am Anfang des Dialogs über Vorteile von WhatsApp geschrieben hat, eher eine skeptische Haltung ein und sucht nach Nachteilen des Messengers. In \#25-28 argumentiert sie beispielsweise damit, dass WhatsApp keine bessere Alternative für SMS sei, da man für die WhatsApp-Nutzung ebenso monatlich zahlen müsse. Schreiber $B$ korrigiert aber $A$ 's Wissen über den Messenger, indem er ihr erklärt, dass man einmal pro Jahr und nicht einmal pro Monat zahlen muss. ${ }^{118}$ Schreiberin $A$ quittiert das Erhalten der Information zwar mit einem Erkenntnisprozessmarker $a$ (,achso'), gibt aber nicht auf, und die Diskussion setzt sich im weiteren Verlauf des Dialogs fort. Die in \#28 durchgeführte selbstinitiierte Selbstkorrektur richtet sich auf zwei Problemquellen aus \#26. Dabei lässt sich das erste Reparandum „чл“, das lediglich eine bedeutungslose Konsonantenverbindung darstellt, als (ortho)graphische Problemquelle einstufen, während das zweite Reparandum „Смог“ (,können/schaffen-SG.MASK.PRÄT“ bzw. ,Smog`), das für ein perfektives Verb in der Vergangenheit oder für ein Nomen steht, sowohl als lexiko-semantisch als auch als (ortho)graphisch angesehen werden kann. Die beiden Reparanda werden in \#28 jeweils durch das Pronomen что (,was') ersetzt, wobei die Nomen „смс“ (,SMS`) und „ватсапп“ (,WhatsApp“) als syntaktische Anker fungieren.

Eine weitere Substitution, bei der aber die Anbindung der Reparaturoperation nicht wie in (143) mithilfe des syntaktischen Ankers, sondern durch die Wiederaufnahme des negierten Reparandums (vgl. Kap. 6.1.1.3) erfolgt, findet sich in Auszug (144):

118 Seit 2016 ist die Nutzung von WhatsApp kostenlos geworden. 
(144) Auszug aus dem WhatsApp-Dialog \#4045:

Schreiberin $A$

Schreiberin $B$

$\rightarrow$

\begin{tabular}{|c|}
\hline Feliz cumpleaños! Espero que los pases súper bien \\
\hline Nachricht \#1 - 16.04.2016 - 14:13:05 \\
\hline
\end{tabular}

\begin{tabular}{|c|}
\hline Ist das richtig so? \\
\hline Nachricht \#2 $-16.04 .2016-14: 13: 05$ \\
\hline
\end{tabular}

$\rightarrow$

\begin{tabular}{|c|}
\hline Lo, nicht los \\
\hline Nachricht \#3 $-16.04 .2016-14: 13: 05$ \\
\hline
\end{tabular}

\begin{tabular}{|c|}
\hline Lo \\
\hline Nachricht \#4 $-16.04 .2016-14: 14: 05$ \\
\hline
\end{tabular}

$\mathrm{Zu}$ Beginn des Dialogs bittet Schreiberin $A$ ihre Freundin, die lateinamerikanische Wurzeln hat und über Spanischkenntnisse verfügt, Nachricht \#1 auf ihre Korrektheit zu überprüfen. Daraus, dass \#1 den spanischen Text und \#2 die Bitte selbst enthält, ergibt sich die Position der in \#3 realisierten selbstinitiierten Selbstkorrektur. Analog zur bereits in 6.1.1.3 beschriebenen Selbstreparatur im next opportunity space, die ebenso eine verneinte Problemquelle miteinschließt (vgl. (116)), wird das Reparans vor dem negierten Reparandum platziert. Das von $A$ vorgelegte Reparans wird von $B$ in \#4 bestätigt.

Während bei den in (143) und (144) durchgeführten Reparaturen im after next opportunity space das Reparandum auf die eine oder andere Weise kenntlich gemacht wird, bleibt in (145) eine Anbindung der Reparaturoperation komplett aus:

(145) Auszug aus dem WhatsApp-Dialog \#3356:

Schreiberin $A$

Schreiberin $B$

$\rightarrow$

Мне тоже сначала бюрократы впихнуть, я думаю делаю то я, и показывать буду я

Sie Bürokraten mir das zuerst auch aufhalsen, ich habe mir gedacht, wenn ich es schon mache, dann will ich es auch selbst zeigen

Nachricht \#15 - 29.04.2014 - 14:27:05

Ясноты как классный руководитель делаешь что нибудь?

Alles klar, und machst du etwas als Klassenlehrerin?

Nachricht \#16 - 29.04.2014 - 14:29:05 


\begin{tabular}{|c|}
\hline Да слежу за их работой с ветеранами \\
\hline Ja, ich betreue ihre Arbeit mit Veteranen mit \\
\hline Nachricht \#17 - 29.04.2014-14:30:05 \\
\hline
\end{tabular}

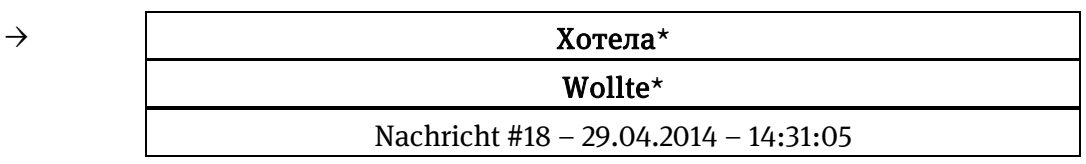

\begin{tabular}{|c|}
\hline Ясноты документы делала какие нибудь? \\
\hline $\begin{array}{c}\text { Okay, hast du schon irgendwelche Unterlagen für den } \\
\text { Praktikumsbericht vorbereitet? }\end{array}$ \\
\hline Nachricht \#19-29.04.2014 - 14:32:05 \\
\hline
\end{tabular}

Bei der Besprechung ihrer Schulpraktika gehen Schreiberinnen $A$ und $B$ u.a. darauf ein, dass die beiden je eine PowerPoint-Präsentation für ihre Praktikumsbetreuerin anfertigen sollen. Dabei hat sich aber Schreiberin $B$ durchgesetzt und das Recht ausgehandelt, ihre Präsentation selbst zu zeigen, wovon sie in \#15 berichtet. Obwohl das in \#15 vorliegende Reparandum „бюрократы“ (,Bürokraten') weder semantisch noch syntaktisch in die Nachricht integriert ist, zeigt $A$ in \#16 trotzdem mit dem Responsiv ясно (,alles klar) ihr Verstehen an und stellt die nächste Frage an $B$. Erst nachdem $B$ auf diese Frage reagiert hat, initiiert sie eine mit dem nachgestellten Asterisk markierte Selbstkorrektur. Es muss jedoch betont werden, dass die von $B$ unternommene Reparaturdurchführung zwar keine Interpretationsprobleme bei $A$ ausgelöst zu haben scheint, diese aber dennoch keine im gegebenen Kontext optimale Problembearbeitung darstellt, da in \#18 die Anbindung der Reparaturoperation ausbleibt, sodass sich das Lokalisieren des Reparandums - zumindest aus der Beobachterperspektive - als schwierig erweist und etwas Zeit in Anspruch nimmt.

Die letzte hier diskutierte selbstinitiierte Selbstreparatur im after next opportunity space bezieht sich auf ein auf der pragmatischen Ebene angesiedeltes Reparandum und zeigt, dass eine solche Reparatur nicht nur in der übernächsten Nachricht des Produzenten der Problemquelle, sondern auch viel später erscheinen kann:

(146) Auszug aus dem WhatsApp-Dialog \#2623:

Schreiber $A$ Schreiberin $B$ $\rightarrow$

\begin{tabular}{|c|}
\hline Hai süsse, sry wegen letztens;) \\
\hline Nachricht \#9- 22.07.2013- 17:47:05 \\
\hline
\end{tabular}




\begin{tabular}{|c|}
\hline Hab heute 2 klausuren geschrieben \\
\hline Nachricht \#10 - 22.07.2013 - 17:47:28 \\
\hline
\end{tabular}

\begin{tabular}{|c|}
\hline Wie war der urlaub? \\
\hline Nachricht \#11 - 22.07.2013 - 17:47:40 \\
\hline
\end{tabular}

\begin{tabular}{|c|}
\hline Und wie war? \\
\hline Nachricht \#12-22.07.2013 - 18:31:55 \\
\hline
\end{tabular}

\begin{tabular}{|c|}
\hline Die eine naja und die andere gut \\
\hline Nachricht \#13 - 22.07.2013 - 20:09:12 \\
\hline
\end{tabular}
süsse geschrieben..

Nachricht \#14 - 22.07.2013 - 20:18:08

\begin{tabular}{|c|}
\hline Hmmm ich weiß du würdest mir niemals aus Eigeninitiative schreiben :( \\
\hline Nachricht \#15 - 22.07.2013 - 21:38:27 \\
\hline
\end{tabular}

Nachricht \#15 - 22.07.2013 - 21:38:27

\begin{tabular}{|c|}
\hline Doch NAME... boah mach mir kein schlechtes gewissen \\
\hline Nachricht \#16 - 22.07.2013 - 21:40:54 \\
\hline Hahahahahaa war nur Spaß \\
\hline Nachricht \#17 - 22.07.2013 - 21:41:05 \\
\hline
\end{tabular}

Das Problem liegt dabei darin, dass die von $B$ verschickten Nachrichten \#9 bis $\# 11$ an den falschen Adressaten geschickt wurden, wobei $B$ dies im Gegensatz zu (99) nicht sofort merkt. Schreiber $A$, der anhand der Anrede „süsse“ (\#9) sowie möglicherweise der Entschuldigung (\#9) und der Frage nach dem Urlaub bereits ahnen könnte, dass die Nachrichten nicht an ihn gerichtet sind, thematisiert das vorliegende Problem jedoch nicht, sondern reagiert mit einer Frage zu Nachricht \#10. Schreiberin $B$ liefert darauf eine erwartbare Antwort und geht erst danach zu einer selbstinitiierten Selbstreparatur über, in der sie erklärt, dass sie $A$ mit einer anderen Person verwechselt hat (,ich dachte du waerst jemand anderes vorhin“). Während die Anredeform „süsse“ von $B$ explizit zurückgenommen wird, geht sie auf die zwei anderen Nachrichten (\#10 und \#11) nicht ein. 


\subsubsection{Missverständnisse im next und after next opportunity space}

Missverständnisse, d.h. selbstinitiierte Selbstreparaturen, deren Notwendigkeit der Produzent der Problemquelle erst an der Reaktion des Rezipienten erkennt (vgl. third position repairs im Sinne von Schegloff 1992; Egbert 2009: 125-156), machen einen vergleichbar geringen Anteil in der gesamten Kollektion von selbstinitiierten Selbstreparaturen aus. Im russischen Korpus sind Missverständnisse mit vier und im deutschen mit 17 Fällen vertreten, was jeweils ca. 7\% und $8 \%$ aller selbstinitiierten Selbstreparaturen entspricht.

Während Missverständnisse in den russischen Kurznachrichtendialogen ausschließlich in der nächsten für die Reparatur zur Verfügung stehenden Position, nämlich im next opportunity space, erscheinen, lässt sich in den deutschen Beispielen zwar in den meisten Fällen die gleiche Position nachweisen, dennoch finden sich auch zwei Missverständnisse im after next opportunity space. Eine solche sequenzielle Position der Reparatur ergibt sich beispielsweise in (147) dadurch, dass die Reparaturdurchführung durch eine reciprocal oder return sequence im Sinne von Schegloff (2007: 195-207) verschoben wird (vgl. \#13-14):

(147) Auszug aus dem Dialog \#2320:

Schreiberin $A$

Schreiber $B$

\begin{tabular}{|c|}
\hline Hey \\
\hline Nachricht \#1 - 12.10.2013 - 12:17:00 \\
\hline
\end{tabular}

\begin{tabular}{|c|}
\hline Mein Baby \\
\hline Nachricht \#2-12.10.2013-12:17:00 \\
\hline
\end{tabular}

\begin{tabular}{|c|}
\hline Nice :D \\
\hline Nachricht \#3 - 12.10.2013 - 12:17:00 \\
\hline
\end{tabular}

\begin{tabular}{|c|}
\hline Bei dir läuft L \\
\hline Nachricht \#4-12.10.2013 - 12:21:00 \\
\hline
\end{tabular}

\begin{tabular}{|c|}
\hline :DD \\
\hline Nachricht \#5 - 12.10.2013-12:21:00 \\
\hline
\end{tabular}

\begin{tabular}{|c|}
\hline :D \\
\hline Nachricht \#6-12.10.2013-12:34:00 \\
\hline
\end{tabular}




\begin{tabular}{|c|}
\hline Langweilig \\
\hline Nachricht \#7 - 12.10.2013 - 12:57:00 \\
\hline
\end{tabular}

\begin{tabular}{|c|}
\hline Ich gucke \\
\hline Nachricht $\# 8-12.10 .2013-12: 57: 00$ \\
\hline
\end{tabular}

\begin{tabular}{|c|}
\hline Walt \\
\hline Nachricht \#9 $-12.10 .2013-12: 57: 05$ \\
\hline
\end{tabular}

$\rightarrow$

\begin{tabular}{|c|}
\hline Wo bist du? \\
\hline Nachricht \#10 - 12.10.2013 - 12:57:05 \\
\hline
\end{tabular}

$\rightarrow$

\begin{tabular}{|c|}
\hline Zu Hause Baby \\
\hline Nachricht \#11 - 12.10.2013 - 13:06:05 \\
\hline
\end{tabular}

\begin{tabular}{|c|}
\hline $\mathrm{Du}$ \\
\hline Nachricht \#12 $-12.10 .2013-13: 06: 05$ \\
\hline
\end{tabular}

\begin{tabular}{|c|}
\hline Uni \\
\hline Nachricht \#13-12.10.2013-14:09:05 \\
\hline
\end{tabular}

\begin{tabular}{|c|}
\hline Langweilig \\
\hline Nachricht \#14 - 12.10.2013 - 14:09:05 \\
\hline
\end{tabular}

$\rightarrow$ Aber ich meinte bei Walt :D

Nachricht \#15 - 12.10.2013 - 14:09:05

\begin{tabular}{|c|c|}
\hline \multirow[t]{2}{*}{$\rightarrow$} & Achso \\
\hline & Nachricht \#16 - 12.10.2013 - 16:15:05 \\
\hline \multirow[t]{2}{*}{$\rightarrow$} & :D \\
\hline & Nachricht \#17 - 12.10.2013-16:15:05 \\
\hline \multirow[t]{2}{*}{$\rightarrow$} & Walt hat gerade den Tipp gegeben \\
\hline & Nachricht \#18 - 12.10.2013 - 16:15:05 \\
\hline
\end{tabular}


Dass dieser Heisenberg doch nicht tot ist

Nachricht \#19 - 12.10.2013 - 16:15:05

Zu Beginn des Dialogs teilt Schreiberin $A$ mit, dass sie sich gerade langweilt, während $B$ im Gegenzug berichtet, dass er „Walt“ schaut, wobei der Name Walt metonymisch für den Serientitel Breaking Bad verwendet wird. Die sich daran anschließende Frage „Wo bist du? “ wird von $B$ allerdings nicht mit dieser Serie, sondern mit seinem aktuellen Standort in Zusammenhang gebracht. Daher liefert $B$ in \#11 eine aus seiner Perspektive sinnvolle Antwort „Zu Hause Baby“ und richtet dieselbe Frage an $A$, produziert also eine return sequence. Schreiberin $A$ verschickt zunächst den zweiten Teil der von $B$ angestoßenen return sequence, bevor sie das vorliegende Missverständnis in \#15 thematisiert. Dabei stellt $A$ mithilfe der adversativen Konjunktion aber einen Gegensatz zu $B$ 's Interpretation ihrer Frage her, welche er durch seine Antwort in \#11 impliziert hat. Anschließend greift $A$ auf das für das Deutsche typische Initiierungsformat ich meinte (vgl. Egbert 2009: 127-128) zurück, gefolgt von der Klarstellung der ursprünglichen Problemquelle durch den Aufbau der Bezüge zur Nachricht \#9 (,bei Walt“). Das nachrichtenfinale Emoticon „:D“ übernimmt dabei eine modalisierende Funktion im Sinne von Pappert (2017: 195-196), indem dieses zur Wahrung des Gesichts sowohl von $A$, deren vage formulierte Frage (\#10) ein Missverständnis ausgelöst hat, als auch von $B$, der diese Frage missverstanden hat, beiträgt (zum Gebrauch eines anderen Emoticons, und zwar :-), bei gesichtsbedrohenden Aktivitäten vgl. Imo 2015b: 150-152). Durch den Erkenntnisprozessmarker achso zeigt $B$ an, dass die von $A$ durchgeführte selbstinitiierte Selbstreparatur seinen Wissensstand verändert hat. Aus diesem Grund reinterpretiert er $A$ 's Frage und liefert in \#18-19 eine neue Antwort darauf, welche dieses Mal $A$ 's kommunikativer Absicht entspricht. Das in der Nachricht davor verschickte Emoticon „:D“ (\#17) könnte man entweder ebenso als modalisierend betrachten oder alternativ als eine Art Kommentar bzw. Bewertung der eigenen Fehlinterpretation deuten (zu Funktionen Modalisierung sowie Kommentierung/Evaluierung vgl. Pappert 2017: 195-198).

Da abgesehen von zwei Fällen in den deutschen Daten Missverständnisse in den Kurznachrichten in beiden hier untersuchten Sprachen in der nächsten Nachricht desselben Schreibers geklärt werden, bezieht sich die folgende Analyse lediglich auf Missverständnisse im next opportunity space. 


\subsubsection{Reparanda}

\section{Problematische Referenzen}

Für Missverständnisse unterscheidet Schegloff (1992) zwei Typen von Reparanda, und zwar diejenigen, die eine problematische Referenz betreffen , und diejenigen, die auf eine problematische sequenzielle Implikation zurückzuführen sind (vgl. auch Egbert 2009: 133-142). In den analysierten Daten finden sich beide Reparandatypen, wobei problematische Referenzen deutlich häufiger repariert werden (jeweils zehn von 15 sowie drei von vier Fällen im deutschen und russischen Subkorpus):

(148) Dialog \#1982:

Schreiberin $A$

Schreiberin $B$

Ich hoffe Du hast mal meinen briefkasten leer gemacht. :) kuss kuss Nachricht \#1 - 08.10.2012 - 09:55

$\rightarrow \begin{gathered}\text { Jajajaja am donnerstag.. Und blumen gegossen. Die waren so komisch } \\ \text { trocken die blätter.. Ist das normal? Also die blumen die für den } \\ \text { balkon sind.. }\end{gathered}$

\begin{tabular}{|c|}
\hline Ja ist normal :) sind schon alt :) kuss \\
\hline Nachricht \#3 $-08.10 .2012-11: 39$ \\
\hline
\end{tabular}

$\rightarrow$\begin{tabular}{c|}
\hline $\begin{array}{c}\text { Ne ich mein die neuen von uns. Die drinnen stehen. Die wir im inet } \\
\text { bestellt haben.. Da sind die blätter so trocken.. }\end{array}$ \\
\hline Nachricht \#4 - 08.10.2012 - 11:41
\end{tabular}

Ja deshalb wollte ich umtopfen die brauchen wohl viel wasser. :) kuss

Nachricht \#5 - 08.10.2012 - 11:55

Ok.dann werd ich die mal wieder giessen heute. Kuss

Nachricht \#6 - 08.10.2012 - 11:56

Ein Missverständnis, das in (148) vorliegt, betrifft die problematische Referenz „blumen“, die in \#2 von $A$ eingeführt wird. Dabei erwähnt $A$, dass sie zwar die Blumen der Mutter gegossen hat, teilt aber $B$ ihre Besorgnis darüber mit, dass einige davon, auf die sie präzisierend mit „die blumen die für den balkon sind“ referiert, trockene Blätter haben. Schreiberin $B$ versichert ihrer Tochter, dass 
das normal sei und sich damit erklären ließe, dass die Blumen „schon alt“ (\#3) sind. Dabei zeigt die Reaktion von $A$ in \#4, dass sie das Adjektiv „alt“ (\#3) offensichtlich als, vor langer Zeit gekauft' interpretiert, während die Mutter sich damit eher auf das ,Lebensalter der Blumen bezieht (vgl. \#5). $A$ fasst allerdings die in \#3 gelieferte Antwort ihrer Mutter als ein Anzeichen eines entstandenen Missverständnisses auf, das sie versucht in \#4 aufzuklären. Zuerst weist sie die vermeintlich auf einem Missverstehen basierende Reaktion ihrer Mutter zurück, dann leitet sie mit „ich mein“ eine Reparatur ein und führt diese anschließend durch, indem sie zu B's Inferenz „schon alt“ (\#3) einen Kontrast aufbaut und mithilfe von zwei attribuierenden Relativsätzen das Reparandum „blumen“ nochmals präzisiert (vgl. Kap. 2.2.2.2). Nachricht \#5 zeigt aber, dass das eigentliche Missverstehen nicht bei $B$, sondern bei $A$ lag, da $A$ die Adjektivgruppe „schon alt“, die sich auf das Nomen „blumen“ (\#2) bezieht, falsch interpretiert hat und daher ein Missverständnis angenommen hat, welches es eigentlich nicht gab. Daher lässt sich (148) als eine Art ,Verschachtelung' von zwei Missverständnissen betrachten, wobei sich das erste (\#2-4) als Produkt des zweiten (\#3-5) herausstellt.

Des Weiteren kann die mit einem Interrogativum erfragte Information anders als von ihrem Produzenten intendiert vom Rezipienten interpretiert werden, was in (149) mit dem Interrogativadverb „сколько“ (,wie viel`) passiert:

(149) Auszug aus dem WhatsApp-Dialog \#3618:

Schreiberin $A$

Schreiberin $B$

$\rightarrow$

\begin{tabular}{|c|}
\hline Хорошо) мы с тобой на сколько в итоге договорились? \\
\hline Geht klar) auf wie viel haben wir uns denn letztendlich geeinigt? \\
\hline Nachricht \#15 - 23.07.2014 - 14:05:05 ${ }^{119}$ \\
\hline
\end{tabular}

\begin{tabular}{|c|}
\hline К 6 примерно \\
\hline So gegen 6 \\
\hline Nachricht \#16 $-23.07 .2014-14: 05: 05$ \\
\hline
\end{tabular}

$\rightarrow$

\begin{tabular}{|c|}
\hline Да я про деньги [Emoji] \\
\hline Ich rede ja übers Geld [Emoji] \\
\hline Nachricht \#17 - 23.07.2014 - 14:05:05 \\
\hline
\end{tabular}

119 Vgl. Fußnote 117. 


\begin{tabular}{|c|}
\hline Aaa \\
\hline Achsoo \\
\hline Nachricht \#18-23.07.2014-14:05:05 \\
\hline
\end{tabular}

In \#3618 geht es darum, dass Schreiberin $B$ einer Freundin $A$ Beeren abkaufen möchte. Es stellte sich jedoch heraus, dass $B$ verreisen muss, sodass sie bis dahin die vorher abgesprochene Menge von Beeren nicht verbrauchen kann und daher zweifelt, ob sich der Kauf für sie lohnt. Nachdem Schreiberin $A$ ihre Freundin doch überzeugt hat, Beeren zu kaufen, stellt $B$ in \#15 eine Frage, die allerdings in der Situation ambig ist. Diese könnte zum einen im Kontext des vermutlich bevorstehenden Treffens als Nachfrage nach dem vereinbarten Termin verstanden werden (,Auf wie viel Uhr haben wir uns denn letztendlich geeinigt?`). Dabei würde man die Präpositionalphrase „на сколько“ als ,на какое время' (,zu welcher Zeit') deuten. Zum anderen besteht die Möglichkeit, dieselbe Präpositionalphrase auf den vorher angesprochenen Preis zu beziehen, sodass „на сколько“ im Sinne von ,на какую сумму“ (,für welche Geldsumme/für welchen Preis ${ }^{\circ}$ ) verwendet wird und die ganze Frage dann folgendermaßen lautet: ,Auf welchem Preis haben wir uns denn letztendlich geeinigt?'. $A$ 's Antwort „К 6 примерно“ (,So gegen $6^{\circ}$ ) impliziert, dass sie sich für die erste Interpretation entscheidet und die Frage in Bezug auf die Zeit des Treffens versteht. In Nachricht \#17 klärt $B$ aber das entstandene Missverständnis auf und legt offen, dass sie sich damit nach dem Preis und nicht nach der Zeit erkundigen wollte. $A$ quittiert zwar in \#18 ihr Verstehen mit dem Erkenntnisprozessmarker „Aaa“ (,achso'), liefert allerdings weder in Anschluss daran noch im weiteren Verlauf des Dialogs eine Antwort in Bezug auf den Preis. Über das Ausbleiben der Antwort kann lediglich spekuliert werden. Da die Zeitangaben in (149) leider nicht miteingegeben wurden, lässt sich nicht eindeutig erkennen, ob der Dialog an dieser Stelle zu Ende war, ob zwischen \#18 und \#19 evtl. ein Telefonat stattfand oder ob die Antwort tatsächlich auch außerhalb des vorliegenden WhatsApp-Dialogs nicht geliefert wurde und die Summe vielleicht erst beim Treffen ausgehandelt wurde.

\section{Problematische sequenzielle Implikationen}

Ein Missverständnis, dessen Entstehen durch das Vorliegen einer problematischen sequenziellen Implikation (problematic sequential implicativeness im Sinne von Schegloff 1992) bedingt ist, liegt dann vor, wenn der Rezipient eine von seinem Gesprächspartner durchgeführte sprachliche Handlung anders als intendiert versteht und dementsprechend darauf reagiert (vgl. Schegloff 1992; Egbert 2009: 133-142). Dabei kann beispielsweise ein an den Rezipienten ge- 
richteter Ratschlag von diesem als Handlungsbekundung des Produzenten interpretiert werden, was in (150) der Fall ist:

(150) Auszug aus dem WhatsApp-Dialog \#4272:

Schreiber $A$

Schreiber $B$

\begin{tabular}{|c|}
$\begin{array}{c}\text { Jo moin } \& \text { was genau habt ihr jetzt eigentlich mit meinem Stick } \\
\text { gemacht? Der zeigt mir trotz formatierung nur } 200 \mathrm{mb} \text { Speicher an, } \\
\text { obwohl der } 16 \text { gb hat }\end{array}$ \\
\hline Nachricht \#1 $-07.07 .2016-13: 40: 42$ \\
\hline
\end{tabular}

$\rightarrow$

:D Hey, frag mal Maggi, ich weiß nicht wie das bei Mac läuft Das gleiche hat er mir auch angezeigt Kommst du Samstag?

Nachricht \#2 - 07.07.2016 - 15:11:42

An manchen PCs funktioniert es garnicht mehr.. Weiß nicht, Maggi hat mir noch nichts gesagt

Nachricht \#3 - 07.07.2016 - 18:33:19

$\rightarrow$ Hat maggi was gesagt?

Nachricht \#4 - 09.07.2016 - 12:14:37

$\rightarrow$

Achso, ich dachte du wolltest ihn anschreiben. Ich frag mal Nachricht \#5 - 09.07.2016 - 12:18:10

\begin{tabular}{|c|c|}
\hline \multirow[t]{2}{*}{$\rightarrow$} & wegen dem \\
\hline & Nachricht \#6 - 09.07.2016 - 12:26:13 \\
\hline & Ja klar, meine ich auch :D \\
\hline & Nachricht \#7 - 09.07.2016 - 12:40:22 \\
\hline & $\mathrm{Ok}: \mathrm{D}$ \\
\hline & Nachricht \#8 - 09.07.2016 - 12:43:10 \\
\hline
\end{tabular}

In \#1 äußert $A$ einen Vorwurf in Bezug darauf, dass $B$ und eine weitere Person $A$ 's USB-Stick auf eine Weise manipuliert haben sollten, dass dieser nicht mehr richtig funktioniert. Schreiber $B$ reagiert auf diesen Vorwurf zunächst mit dem Emoticon „:D“, welches in dem Fall eine modalisierende Funktion ausübt und die für ihn gesichtsbedrohende Handlung abfedert (vgl. Imo 2015b: 150-152; 
Pappert 2017: 196). Danach schlägt $B$ jedoch keine Lösung für $A$ ’s Problem vor, sondern leitet ihn an „Maggi“ weiter und begründet diesen Ratschlag gleichzeitig mit der eigenen mangelnden Expertise bei dem Umgang mit USB-Sticks und MacBooks. Mit der Bestätigung der von $B$ gemeldeten Störung „Das gleiche hat er mir auch angezeigt“ (\#2) schließt $B$ diesen thematischen Abschnitt der Interaktion ab und geht zum nächsten Thema über, indem er eine Frage danach stellt, ob $A$ am Samstag kommt. Daraufhin greift $A$ das Thema mit dem Stick noch einmal auf, indem er sich darüber beschwert, dass sein Stick an einigen PCs nicht mehr geöffnet werden kann. Seine anschließende Bemerkung „Weiß nicht, Maggi hat mir noch nichts gesagt“ bezieht sich höchstwahrscheinlich auf $B$ 's Frage zu dem Treffen am Samstag. Zwei Tage später verschickt $A$ in \#4 eine Nachfrage, welche zeigt, dass $A B$ 's im Imperativ formulierten Ratschlag „frag mal Maggi“ (\#2) als elliptische Struktur mit Verbspitzenstellung (vgl. Auer 1993) im Sinne von ich frage mal Maggi interpretiert und daher auf $B$ 's Rückmeldung gewartet hat. In \#5 signalisiert $B$ zuerst mit achso, dass er das entstandene Missverständnis erkannt hat, dann klärt er dieses mit „ich dachte du wolltest ihn anschreiben“ auf, indem er implizit auf \#2 Bezug nimmt. Letztendlich erklärt sich $B$ bereit, selbst Maggi zu kontaktieren. Die von $B$ somit durchgeführte Reparatur ist allerdings fehlgeschlagen, diese wurde von $A$ nicht als Bearbeitung einer problematischen sequenziellen Implikation, sondern als Anzeichen davon interpretiert, dass $B$ A's Frage in \#4 missverstanden und auf das Treffen am Samstag und nicht auf das Problem mit dem Stick bezogen hat. Daher führt $A$ in \#6 ebenso eine Selbstreparatur durch („Ich meine wegen dem Stick“), um das vermeintliche Missverständnis aufzulösen und die von ihm gemeinte Referenz seinem Kommunikationspartner klarzumachen. Schreiber Bbestätigt in \#7, dass er von derselben Referenz wie $A$ ausgegangen ist (,Ja klar, meine ich auch :D“). Darauf reagiert $A$ zwar mit „Ok :D“, es bleibt aber immer noch unklar, ob er den Grund seines ursprünglichen Missverstehens erkannt hat oder nicht. Im weiteren Verlauf des Dialogs (nicht abgebildet) wird das Problem mit dem Stick weiter besprochen, eine endgültige Lösung wird zwar nicht gefunden, aber in \#14 teilt $A$ mit, dass Maggi ihn gerade diesbezüglich anschreibt.

Eine im Gegensatz zu (150) erfolgreiche Reparatur, die ebenso eine problematische sequenzielle Implikation bearbeitet, liegt in (151) vor:

(151) SMS-Dialog \#2542:

Schreiber $A$

Schreiberin $B$

$\rightarrow$\begin{tabular}{|c|}
\hline [женское имя],мы готовы выйти сейчас. \\
\hline [Frauenname], wir sind bereit, gleich aufzubrechen. \\
\hline Nachricht \#1 - 22.06.2013 - 14:09:00 \\
\hline
\end{tabular}




\begin{tabular}{|c|}
\hline Не рано? За сколько вы доехали туда? \\
\hline $\begin{array}{c}\text { Ist es nicht zu früh? Wie lange habt ihr gebraucht, um dahin zu } \\
\text { kommen? }\end{array}$ \\
\hline Nachricht \#2-22.06.2013 - 14:11:00 \\
\hline
\end{tabular}

\begin{tabular}{|c|}
\hline За 20 мин. Я тебя спрашиваю,или ждать и к 14,50? \\
\hline $\begin{array}{l}20 \text { Minuten. Ich frage dich, sollen wir schon aufbrechen oder sollen wir } \\
\text { warten und gegen 14:50 Uhr kommen? }\end{array}$ \\
\hline Nachricht \#3 - 22.06.2013 - 14:12:00 \\
\hline
\end{tabular}

Давай к 14.50; потому что мы уже в это время договорились)

Lass uns um 14:50 Uhr treffen, da wir diese Zeit ursprünglich vereinbart haben)

Nachricht \#4 - 22.06.2013 - 14:13:00

Мы на месте, на стороне памятника казаку

Wir sind vor Ort, von der Seite, wo das Kosakendenkmal steht

Nachricht \#5 - 22.06.2013 - 14:52:00

Im Rahmen einer Terminvereinbarung zwischen Schreiber $A$ und Schreiberin $B$ signalisiert $A$ in \#1 seine Bereitschaft, sich gleich auf den Weg zum vereinbarten Ort zu machen. Dabei deutet $B$ diese Nachricht als eine Handlungsbekundung, die sie in \#2 jedoch zur Diskussion stellt, da sie vermutet, dass es noch zu früh wäre, um aufzubrechen. Daraufhin macht $A$ in \#3 seine ursprüngliche kommunikative Absicht deutlicher, indem er die intendierte sprachliche Handlung explizit als Frage benennt („Я тебя спрашиваю“ ,Ich frage dich', vgl. die Charakterisierung der Problemquelle wie $I$ was just kidding. bei Schegloff 1992: 1312-1313). Aus dieser Perspektive erhält \#1 somit die Bedeutung ,Wir sind bereit gleich aufzubrechen. Sollen wir es tun?', die mit einer in \#3 gelieferten Alternative („,или ждать и к 14,50?“ ,oder sollen wir warten und gegen 14:50 Uhr kommen?') kontrastiert wird. $B$ bestätigt in \#4 die zweite Option und somit ist das vorliegende Missverständnis behoben.

Neben Fällen, in denen eine sprachliche Handlung als eine andere verstanden wird, unterscheidet Schegloff (1992) auch Missverständnisse, bei denen das Problem an der Interpretation der Interaktionsmodalität liegt, d.h. eine scherzhafte Äußerung wird als ernsthaft aufgenommen oder umgekehrt (vgl. auch Egbert 2009: 133-134). Das einzige in den untersuchten Daten vorliegende Beispiel, das dieser Gruppe zugeordnet werden kann, wird im weiteren Verlauf der Arbeit als (158) diskutiert. 


\subsubsection{Reparaturinitiierungen und -durchführungen}

Die Struktur der Bearbeitung eines Missverständnisses wird im Gegensatz zu den bereits in Kap. 6.1.1-6.1.3 beschriebenen selbstinitiierten Selbstreparaturen von Schegloff (1992: 1304-1308) in vier Komponenten aufgeteilt, von denen allerdings nicht alle zwingend realisiert werden müssen (vgl. auch Egbert 2009: 142). Dazu gehört zunächst die A-Komponente, in der die Reparaturinitiierung produziert wird. Danach folgt die B-Komponente, die i.d.R. bei Beschwerden vorkommt und in deren Rahmen die von der Beschwerde ausgelöste Entschuldigung akzeptiert oder rechtfertigt wird. Im nächsten Schritt kann als C-Komponente eine Zurückweisung der falschen Interpretation erfolgen, bevor die D-Komponente in Form der Reparaturoperation schließlich geliefert wird (vgl. Kap. 2.2.2.2). Im Folgenden soll nun die Realisierung einzelner Komponenten bei Missverständnissen im Korpus vorgestellt werden.

\section{A-Komponente}

Bei 15 Missverständnissen im next opportunity space aus den deutschen Daten enthalten elf mindestens einen Reparaturmarker, der entweder der A-Komponente angehört oder die D-Komponente einleitet (vgl. Egbert 2009: 142). ${ }^{120}$ Dazu gehören zum einen die Partikeln achso (drei Tokens, vgl. (150)), ne(e) (zwei Tokens; vgl. (148) und (159)) und der Diskursmarker also (zwei Tokens; vgl. \#18 in (88)). Zum anderen finden sich in fünf Fällen phrasale und satzwertige Reparaturmarker mit dem Verb meinen im Präsens und Präteritum (fünf Tokens; vgl. (148), (150) und (152)) sowie mit denken im Präteritum (zwei Tokens; vgl. (150)). Einen besonderen Fall stellt (157) dar, in dem die Majuskelschreibung als zusätzliches graphisches Mittel bei der Wiederaufnahme des Reparandums eingesetzt wird, um die Aufmerksamkeit der Rezipientin auf die von ihr falsch gelesene Problemquelle zu lenken und somit die anschließende Reparaturdurchführung anzumoderieren.

Bei den Reparaturinitiierungen mit dem Verb meinen wird dieses in zwei Fällen (vgl. (148) und (150)) im Präsens und in drei im Präteritum realisiert (vgl. (152)):

120 Das eine von zwei Missverständnissen im after next opportunity space enthält ebenso eine A-Komponente. Dabei handelt es sich um den Reparaturmarker ich meinte in Kombination mit der adversativen Konjunktion aber (vgl. (147)). 
(152) Auszug aus dem WhatsApp-Dialog \#3061:

Schreiberin $A$

Schreiberin $B$

\begin{tabular}{|c|}
\hline Wann hast du dann heute frei? \\
\hline Nachricht \#11 - 12.02.2014 - 12:56:05 \\
\hline
\end{tabular}

Tja musste letzte woche für zwei und diese woche auch auch zwei voc.

Tests lernen

Nachricht \#12 - 12.02.2014 - 12:56:05

\begin{tabular}{|c|}
\hline Um 15:00 \\
\hline Nachricht \#13-12.02.2014-12:56:05 \\
\hline
\end{tabular}

$\rightarrow$

\begin{tabular}{|c|}
\hline Englisch? \\
\hline Nachricht \#14 - 12.02.2014 - 12:57:05 \\
\hline
\end{tabular}

Nee arbeitsstunde

Nachricht \#15 - 12.02.2014 - 13:54:05

$\rightarrow$

Ich meinte die Tests

Nachricht \#16 - 12.02.2014 - 14:05:05

Achso Ja englisch

Nachricht \#17 - 12.02.2014 - 14:13:05

Schreiberin $A$ und Schreiberin $B$ sind miteinander verwandt. Schreiberin $A$ geht noch auf die Schule, während $B$ an einer Uni studiert und gerade Ferien hat, wofür sie von $A$ beneidet wird, die sich zum Zeitpunkt des Dialogs in der Schule aufhält. In \#11 knüpft $B$ daran an und fragt, wann $A$ an dem Tag frei hat. Bevor $A$ diese Frage beantwortet, berichtet sie von ihrem Lernen für die Tests. $B$ 's Nachfrage „Englisch?“ (\#14) bringt $A$ mit Nachricht \#13 in Verbindung, sodass sie annimmt, dass $B$ davon ausgeht, dass sie bis 15 Uhr Englischunterricht hat. Daher verneint $A$ in \#15 $B$ 's Annahme, woraufhin $B$ eine Reparatur mit „Ich meinte“ einleitet und den Bezug zu den Tests herstellt. $A$ zeigt ihr Verstehen in Nachricht \#17 an und bestätigt B's Annahme.

Darüber hinaus kann die A-Komponente auch ausbleiben, was im deutschen Subkorpus in fünf Dialogen mit Missverständnissen der Fall ist: 
(153) Auszug aus dem SMS-Dialog \#2109:

Schreiber $A$

Schreiber $B$

$\rightarrow$

Kommste vorher noch zum kopieren zur Bib? Stichwort: VORNAME

Nachricht \#1 - 19.04.2013 - 11:58:54

\begin{tabular}{|c|}
\hline Ich wollt gegen halb eins los, hab aber kein Geld mehr auf der karte \\
\hline Nachricht \#2 - 19.04.2013 - 12:00:01 \\
\hline Ich wollte ja deine Blätter für mich :-) kopieren. \\
\hline Nachricht \#3 - 19.04.2013 - 12:02:10 \\
\hline Achso :-P \\
\hline Nachricht \#4-19.04.2013-12:02:50
\end{tabular}

Auf die in \#1 von $A$ formulierte Frage bezüglich des Kopierens reagiert $B$ mit einer Mitteilung darüber, dass er kein Geld auf seiner Kopierkarte hat, was $A$ zu verstehen gibt, dass seine Nachricht falsch interpretiert wurde. Während $B$ denkt, dass er von $A$ zum Kopieren aufgefordert wurde, wollte $A$ eigentlich $B$ 's Unterlagen für sich kopieren.

Unter vier Missverständnissen im russischen Korpus lässt sich lediglich in einem Fall eine eindeutige Reparaturinitiierung identifizieren:

(154) Auszug aus dem WhatsApp-Dialog \#3635:

Schreiberin $A$

Schreiberin $B$

\begin{tabular}{|c|}
\hline Тань, погнали завтра в кино \\
\hline Tanja, lass uns mal morgen ins Kino gehen \\
\hline Nachricht \#1 - 26.08.2014 - 22:34 \\
\hline
\end{tabular}

$\rightarrow$

\begin{tabular}{|c|}
\hline Погнали:) во сколько? \\
\hline Lass mal machen:) um wie viel Uhr? \\
\hline Nachricht \#2 $-26.08 .2014-22: 34$ \\
\hline
\end{tabular}

Давай после обеда, чтобы поспать

Lieber am Nachmittag, um sich auszuschlafen

Nachricht \#3 - 26.08.2014 - 22:35 


\begin{tabular}{c}
$\qquad \begin{array}{c}\text { ну понятно, что не в } 8 \text { утра:) я думала ты мне скажешь вр } \\
\text { сеанса }\end{array}$ \\
$\begin{array}{c}\text { es ist schon klar, dass nicht um 8 Uhr morgens:) ich dachte, du } \\
\text { mir die Zeit, wann die Vorstellung beginnt }\end{array}$ \\
\hline Nachricht \#4 - 26.08.2014 - 22:35 \\
\hline $\begin{array}{c}\text { a, ну я думала просто в город поедем, а там какой фильм будет, } \\
\text { на такой и пойдём }\end{array}$ \\
\hline $\begin{array}{c}\text { achso, ich dachte ja, dass wir einfach in die Stadt fahren und zu } \\
\text { irgendeinem Film hingehen, der in jenem Moment laufen wird }\end{array}$ \\
\hline Nachricht \#5 - 26.08.2014 - 22:35 \\
\hline
\end{tabular}

Ausschnitt (154) weist eine zu einem deutschen Reparaturmarker ich dachte parallele Konstruktion auf, und zwar „я думала“ (\#4). Diese bezieht sich auf ein Missverständnis bezüglich der Zeitangabe, die im Kontext einer Verabredung für einen gemeinsamen Kinobesuch zwischen Schreiberinnen $A$ und $B$ in \#2 erfragt wird. Dabei bringt $A B$ 's Frage „во сколько?“ (,Um wie viel Uhr?’) mit der Zeit des Treffens in Verbindung und schlägt den Nachmittag als Termin vor. Daraufhin merkt $B$ in \#4 an, dass es sich von selbst versteht, dass sie nicht am frühen Morgen ins Kino gehen, und präzisiert, dass sich ihre Frage auf die Zeit einer Kinovorstellung bezog. In \#5 quittiert $A$ den Erhalt der neuen Information und rechtfertigt ihre Antwort damit, dass sie an keinen konkreten Film gedacht hat, sondern eher dafür wäre, sich einen spontan auszusuchen.

\section{B-Komponente}

Schegloff (1992: 1304-1308) erwähnt das Vorhandensein der B-Komponente in Form einer Akzeptanz v.a. im Kontext von Beschwerden (vgl. auch Egbert 2009: 142). In ihrer Untersuchung von Foren-Beiträgen, die das Verb missverstehen enthalten, weitet Salomonsson (2011: 89) den Geltungsbereich der Akzeptanz aus und versteht unter B-Komponente jede Art von Akzeptanz, die das Vorliegen eines Verstehensproblems bestätigt. Diese Akzeptanz, die beispielsweise mit „OK“ oder ,ja ich weiß“ zum Ausdruck gebracht werden kann, hat laut Salomonsson (2011: 89) mit Beziehungsarbeit zu tun. In den analysierten deutschen Daten könnte der Erkenntnisprozessmarker achso (drei Tokens; vgl. (150)) als B-Komponente im weiteren Sinne (vgl. Salomonsson 2011: 89) angesehen werden. Im russischen Teilkorpus weist der Abschnitt „ну понятно, что не в 8 утра:)“ (,es ist schon klar, dass nicht um 8 Uhr morgens:)') aus der bereits in (154) diskutierten Reparatur die meisten Anknüpfungspunkte mit dem Konzept der Akzeptanz auf. Auf diese Weise wird $A$ 's Idee, zu einer Nachmittagsvor- 
stellung zu gehen, um sich ausschlafen zu können, die aus der falschen Interpretation von $B$ ’s Frage „во сколько?“ (,Um wie viel Uhr?`) erwachsen ist, ironisch angenommen. Man könnte in dem Fall sogar beinahe von einer Frotzelaktivität ausgehen, in deren Rahmen $B$ ihre Kommunikationspartnerin durch den mit „в 8 утра“ (,um 8 Uhr morgens`) aufgebauten Kontrast sanft aufzieht.

\section{C-Komponente}

In der C-Komponente erfolgt die Zurückweisung der durch die Reaktion des Rezipienten explizit oder implizit dargelegten Interpretation der Nachricht mit der Problemquelle. Im Russischen lässt sich die Zurückweisung zum einen mit der Negationspartikel не (,nicht') realisieren. Zum anderen kann die Ellipse не об этом (wortwörtlich ,nicht über das'; sinngemäß ,das meine ich nicht‘), die für я не об этом говорю (,ich rede nicht darüber') steht (vgl. Goletiani 2003: 237-240, 352), ebenso in der gleichen Funktion eingesetzt werden:

(155) Auszug aus dem WhatsApp-Dialog \#3618:

\begin{tabular}{|c|c|}
\hline \multirow{4}{*}{$\rightarrow$} & in $A$ \\
\hline & А на сколько вы едете в Питер \\
\hline & Und für wie lange fahrt ihr nach Sankt Petersburg? \\
\hline & Nachricht \#6 - 23.07.2014 - 14:05:05 ${ }^{121}$ \\
\hline & В 21:20 поезд \\
\hline & Um 21:20 fährt der Zug ab \\
\hline & Nachricht \#7 - 23.07.2014 - 14:05:05 \\
\hline$\rightarrow$ & Не об этом \\
\hline & Das meine ich nicht \\
\hline & Nachricht \#8 - 23.07.2014 - 14:05:05 \\
\hline$\rightarrow$ & Как долго вы там будете \\
\hline & Wie lange bleibt ihr da? \\
\hline & Nachricht \#9-23.07.2014-14:05:05 \\
\hline
\end{tabular}

Ausschnitt (155) stammt aus dem gleichen Dialog wie bereits in (149) vorgestelltes Beispiel: Es geht dabei darum, dass Schreiberin $B$ gerade ihre Entscheidung anzweifelt, Beeren bei $A$ für ihren Freund zu kaufen, da die beiden demnächst

121 Vgl. Fußnote 117. 
nach Sankt Petersburg fahren und daher bis dahin die Beeren nicht aufessen können. Schreiberin $A$ versucht ihrerseits Argumente zu finden, um $B$ doch in der Richtigkeit ihrer Kaufentscheidung zu überzeugen. In Nachricht \#6 leitet $A$ eines der Argumente ein, welches darauf abzielen sollte, dass Schreiberin $B$ im Falle eines kurzen Aufenthalts in Sankt Petersburg die Beeren auch nach ihrer Rückkehr verbrauchen könnte. Auf $A$ 's Frage nach der Dauer ihres Aufenthalts in Sankt Petersburg liefert $B$ aber eine Antwort, die offenlegt, dass sie höchstwahrscheinlich anstatt „на сколько“ (,für wie lange‘) во сколько (,wann, um wie viel Uhr') gelesen hat. In \#8 zeigt $A$ durch eine Zurückweisung von $B$ 's Antwort, dass ihre Frage nicht wie beabsichtigt verstanden wurde, splittet ihre Reparatur in zwei Nachrichten auf und liefert die Reparaturdurchführung (D-Komponente) in \#9, indem sie ihre ursprüngliche Frage umformuliert (vgl. Schegloff 1992: 1310-1311). Dabei hebt $A$ durch die Formulierung „Как долго“ (,wie lange`) den für ihre Argumentation relevanten Aspekt der Reisedauer noch einmal hervor. Allerdings wartet $A$ nicht mehr die Reaktion von $B$ ab, welche das wiederhergestellte gegenseitige Verstehen signalisiert, sondern geht zum nächsten Schritt ihrer Argumentation über und schlägt vor, einen Teil von den Beeren mitzunehmen und den Rest einzufrieren. Danach wendet $B$ zwar ein, dass ihr Freund eingefrorene Beeren nicht mag, und erkundigt sich, ob sie evtl. nur ein Kilo Beeren kaufen könnte, stimmt dem Kauf dennoch implizit zu.

Für das Deutsche hat Egbert (2009:147) eine Reihe von Formaten beschrieben, mit denen Sprechern C-Komponenten bei unterschiedlichen Problemquellen gestalten können. Bei problematischen Referenzen finden sich Floskeln wie ich meinte $Y$ nicht $X$, das heißt nicht $X$ oder so ist das nicht gemeint, während problematische sequenzielle Implikationen in Form von ich will nicht $X$-en zurückgewiesen werden (vgl. Egbert 2009: 147). In den 15 analysierten Missverständnissen im next opportunity space aus den deutschen Daten liegen zwei Fälle von Zurückweisungen vor. In einem Fall wird die C-Komponente durch nicht $X$ realisiert, während in (156) eine weitere Zurückweisung in Form eines regulär gebildeten Satzes, allerdings ebenso mit der Negationspartikel nicht, erfolgt:

(156) WhatsApp-Dialog \#3395:

Schreiberin $A$

Schreiberin $B$

\begin{tabular}{|c|}
\hline Möchtest du dahin gehen? :) \\
\hline Nachricht \#1 $-25.03 .2014-21: 25: 05$ \\
\hline
\end{tabular}

Kommst du mit?

Nachricht \#2 - 25.03.2014 - 21:27:05 


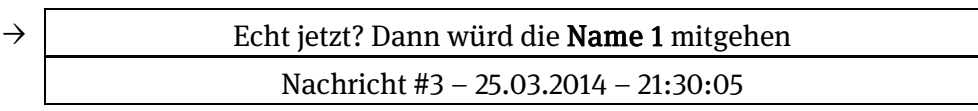

\begin{tabular}{|c|}
\hline Oh,Sorry ich glaube ich habe Kopfweh \\
\hline Nachricht \#4 - 25.03.2014 - 21:31:05 \\
\hline Aber du hast ihm nicht mit Herrn Name 2 verwechselt oder? :) \\
\hline Nachricht \#5 - 25.03.2014 - 21:32:05 \\
\hline Doch,glaube schon. Ist er das nicht? \\
\hline Nachricht \#6 - 25.03.2014 - 21:33:05 \\
\hline Wer lesen kann ist klar im Vorteil! !!! NAME 1 nicht Name 2 \\
\hline Nachricht \#7 - 25.03.2014 - 21:35:05 \\
\hline Ja genau :) aber war ja auch nur ein Buchstabe :) \\
\hline Nachricht \#8 - 25.03.2014 - 21:38:05 \\
\hline
\end{tabular}

Schreiberin $A$ erkundigt sich bei ihrer Verwandten, Schreiberin $B$, ob $B$ irgendwohin gehen wollte. Dabei zeigt das deiktisches Adverb „dahin“, dass das in (156) thematisierte Treffen bzw. die Veranstaltung vorher bereits Thema war. Schreiberin $B$ gibt allerdings keine eindeutige Antwort auf $A$ 's Frage, sondern stellt eine Gegenfrage danach, ob $A$ mitkommt. Diese Gegenfrage könnte sowohl eine implizite Bestätigung als auch eine noch nicht getroffene Entscheidung signalisieren, die in Abhängigkeit von $A$ 's Antwort positiv oder negativ ausfallen könnte. Mit der Floskel „Echt jetzt?“ (\#3), deren Funktion nach Imo (2010: 47) in „der Überprüfung der Interaktionsmodalität“ besteht, hinterfragt $A$ die Ernsthaftigkeit von $B$ 's Frage und begründet dies dadurch, dass, wenn sie mitkommen würde, auch eine andere Person mit ihr mitkäme. Die von $A$ in \#3 erwähnte Person verwechselt $B$ aber mit einem Mann, dessen Anwesenheit bei der Veranstaltung für sie anscheinend unangenehm wäre, was sie durch die in \#4 vorliegende scherzhafte Ausrede signalisiert. Anhand dieser negativen Reaktion vermutet Schreiberin $A$ aber, dass $B$ die von ihr gemeinte Person mit jemand anderem verwechselt hat, sodass $A$ in $\# 5$ explizit darauf eingeht. ${ }^{122} B$ stimmt in

122 Dadurch, dass das Pronomen ,ihm“ (sic!) in dem Fall auf Name 1 aus \#3 verweist, entsteht eine Diskrepanz zwischen diesem Namen, bei dem der vorangestellte Artikel die eigentlich eine weibliche Person impliziert, und dem auf eine männliche Person referierenden Personalpronomen „ihm“, das allerdings nicht im Akkusativ, sondern im Dativ steht. Hinsichtlich des 
\#6 A's Annahme zu und zeigt in \#7 an, dass sie die Verwechslung erkannt hat. Der Grund für das entstandene Missverständnis liegt darin, dass $B$ die Namen, die sich - wie $A$ in \#8 anmerkt - voneinander nur minimal unterscheiden, verwechselt hat.

\section{D-Komponente}

Für die D-Komponente bzw. die Reparaturdurchführung unterscheidet Schegloff (1992: 1308-1312) sechs Reparaturoperationen, und zwar die Wiederholung der problematischen Äußerung (vgl. ,saying it more clearly“ bei Schegloff 1992: 1308), die Charakterisierung der Problemquelle sowie weitere vier Operationen, die i.d.R. in Kombination mit I mean vorkommen: die Kontrastierung, die Umformulierung, die Spezifizierung und die Erklärung der Problemquelle.

Eine explizite Wiederholung der Problemquelle findet sich als Reparaturoperation bei Missverständnissen in den untersuchten Daten äußerst selten, und zwar lediglich in einem Beleg aus dem deutschen Subkorpus (vgl. (157)). Diese Tatsache lässt sich vermutlich damit begründen, dass, wenn eine problematische Äußerung im Rahmen einer Reparaturdurchführung erneut geliefert wird, der Produzent der Problemquelle davon ausgeht, dass der Rezipient die ursprüngliche Äußerung missinterpretiert hat, da er diese nicht korrekt rezipiert hat. In der gesprochenen Sprache kann ein Teil der ursprünglichen Äußerung beispielsweise überhört oder falsch gehört werden, während in der medial schriftlichen Kommunikation der Leseprozess gestört werden könnte (vgl. (156) und (157)). In der Kurznachrichtenkommunikation haben Rezipienten zwar potenziell die Möglichkeit, die abgespeicherten, schriftlich realisierten Daten mehrmals zu rezipieren und somit ein Missverständnis zu vermeiden, gleichzeitig sind sie aber i.d.R. darauf angewiesen, eine Nachricht nebenbei zu lesen und auf diese möglichst schnell zu reagieren, sodass die Rezeptionszeit u.U. verringert wird, was zum ,Verlesen' führen kann:

somit vorliegenden Widerspruchs können zwei Hypothesen formuliert werden. Zum einen könnte bei „die Name 1“ (\#3) wirklich eine weibliche Person gemeint sein, deren Namen sich nur in einem Buchstaben von einem männlichen Namen 2 unterscheidet (vgl. Heike - Heiko). In diesem Fall sollte anstelle des Pronomens ,ihm“ in \#5 das Pronomen sie stehen, das aber aus irgendeinem Grund wie Eingriff der Autokorrektur oder ,Freud'scher Verschreiber' falsch realisiert wurde. Zum anderen ist es nicht ausgeschlossen, dass sich „Name 1“ und „ihm“ auf eine männliche Person beziehen. Dabei würde der Artikel die bei „die Name 1“ (\#3) fehl am Platz sein, da es der Name 1 heißen soll. Da die Namen leider beim Einstellen in die Datenbank anonymisiert wurden, kann dieses Dilemma nicht gelöst werden. 
(157) SMS-Dialog \#1280:

\begin{tabular}{l}
$\rightarrow \quad$ Schreiberin $A$ \\
\hline Noch 30 minuten vorlesung.kauf mal kein ticket. \\
\hline Nachricht \#1 - 26.10.2011 - 15:46:20 \\
\hline Für wann?samstag?wenn ja,wie spät soll ich dann da sein? \\
\hline Nachricht \#2 - 26.10.2011 - 15:47:30 \\
$\rightarrow$ KEIN,ich wiederhole KEIN ticket :-D hab anderen plan.mehr infos in \\
kürze. \\
\hline Nachricht \#3 - 26.10.2011 - 15:48:10
\end{tabular}

\begin{tabular}{|c|}
\hline Oh,ich hab das k überlesen.also nicht. :-D \\
\hline Nachricht \#4 - 26.10.2011 - 15:48:50 \\
\hline
\end{tabular}

Der einzige Fall im Korpus, in dem Wiederholung als Reparaturoperation auftritt und als solche auch explizit bezeichnet wird, liegt in Dialog (157) vor, der zwischen zwei Geschwistern verläuft. Der Nachricht \#1 geht ein Anruf von $B$ voran, den $A$ allerdings nicht annehmen konnte, da sie sich gerade in einer Vorlesung befindet. Den nicht von ihr entgegengenommenen Anruf rechtfertigt $A$ in \#1 und gibt außerdem $B$ eine Anweisung, kein Ticket zu kaufen. Aus $B$ 's Reaktion in \#2, in der sie sich nach einem genaueren Zeitpunkt für die Fahrt erkundigt, lässt sich schließen, dass sie $A$ 's Anweisung genau umgekehrt verstanden hat und vorhat, sich ein Ticket zu besorgen. Das durch ein von $B$ übersehenes $k$ entstandene Missverständnis (vgl. \#4) bearbeitet $A$ in \#3, indem sie das Pronomen kein wiederholt (und zwar sogar zweimal) und dieses mithilfe der durchgehenden Majuskelschreibung graphisch prominent macht. Dabei bringt sie durch „ich wiederhole“ zum einen die Art der vorliegenden Reparaturdurchführung zum Ausdruck und zum anderen macht sie $B$ auf Nachricht \#1 aufmerksam. $B$ reagiert in \#4 mit einem Erkenntnisprozessmarker (vgl. Imo 2009), rechtfertigt ihre Fehlinterpretation und hält abschließend als eine Art Fazit fest, dass doch kein Ticket gekauft werden muss.

Mit der Charakterisierung der Problemquelle bringt Schegloff (1992: 13121313) in erster Linie die Klarstellung der Interaktionsmodalität in Verbindung, also einen expliziten Verweis mit Floskeln wie I'm not kidding. oder I was just kidding. darauf, ob die produzierte Äußerung als ernsthaft oder als scherzhaft gedacht ist (vgl. auch Egbert 2009: 148-150): 
(158) Auszug aus dem WhatsApp-Dialog \#2145:

Schreiber $A$

Schreiber $B$

\begin{tabular}{|c|}
\hline Ciao bis morgen \\
\hline Nachricht \#18 - 02.05.2013-23:20:00 \\
\hline
\end{tabular}

$\rightarrow$

Ich drück dir die daumen

Nachricht \#19-02.05.2013-23:21:00

\begin{tabular}{|c|}
\hline Bist heute aber lustig... -.- \\
\hline Nachricht \#20 - 02.05.2013 - 23:21:00 \\
\hline
\end{tabular}

$\rightarrow$

\begin{tabular}{|c|}
\hline Das ist kein witz \\
\hline Nachricht \#21 - 02.05.2013- 23:21:00 \\
\hline
\end{tabular}

\begin{tabular}{|c|}
\hline Jaja... \\
\hline Nachricht \#22 - 02.05.2013 - 23:21:00 \\
\hline
\end{tabular}

In (158) wird im Rahmen einer Reparatur Nachricht \#19 als ernst gemeint eingestuft, obwohl Schreiber $A$ diese anders wahrnimmt. Die dabei als Problemquelle auftretende Floskel „Ich drück dir die daumen“ bezieht sich auf die von $A \mathrm{im}$ vorherigen Verlauf des Dialogs geäußerte Beschwerde über seinen kaputten Laptop, den er für sein Studium dringend braucht. Nachdem $A$ ankündigt, dass er jetzt ohne Laptop nichts machen kann und daher schlafen geht, und sich in \#18 von $B$ verabschiedet, verschickt $B \mathrm{ihm}$ die ermunternde Floskel „Ich drück dir die daumen". $A$ empfindet diese aber offensichtlich als eine Frotzelaktivität (vgl. \#20), sodass $B$ in \#21 seine vorangehende Nachricht explizit als ernsthaft positionieren muss, was für $A$ allerdings immer noch nicht glaubwürdig genug zu sein scheint (vgl. seine Antwort „Jaja...“ in \#22).

Obwohl Schegloff (1992: 1312-1313) nur die Reparatur der Interaktionsmodalität als Charakterisierung der Problemquelle betrachtet, könnte es m.E. gewinnbringend sein, den Anwendungsbereich dieser Reparaturoperation zu erweitern und auf Reparaturdurchführungen zu übertragen, bei denen eine realisierte sprachliche Handlung explizit genannt wird. Eine solche Erweiterung würde es erlauben, solche Reparaturdurchführungen wie „Я тебя спрашиваю“ (,Ich frage dich') aus (151), in der eine Problemquelle ausdrücklich als eine Frage und nicht als eine Absichtsbekundung bezeichnet wird, als Charakterisierung der Problemquelle (vgl. Schegloff 1992: 1308-1312) aufzufassen. 
Die vier weiteren Reparaturoperationen (die Kontrastierung, die Umformulierung, die Spezifizierung oder die Erklärung der Problemquelle) lassen sich ebenso in den untersuchten Missverständnissen wiederfinden. Während in den Daten von Schegloff (1992) diese Operationsformate v.a. mit der Reparaturinitiierung I mean gekoppelt sind, hat die Analyse gezeigt, dass diese auch auf Reparaturdurchführungen mit anderen Reparaturmarkern bzw. sogar ohne explizite Reparaturmarker übertragbar sind. Als Kontrastierung der Problemquelle kann die in (156) diskutierte Reparaturdurchführung angesehen werden, in der der Name der gemeinten Person dem Namen der anderen mit ihr verwechselten Person gegenübergestellt wird. Ein klarer Fall für die Umformulierung der Problemquelle tritt in (155) auf (на сколько (,für wie lange`) $\rightarrow$ как долго (,wie lange $\left.^{6}\right)$, in dem die eine Zeitspanne erfragende Kombination aus der Präposition на (,für') und dem Adverb сколько (,wie lange, wie viel') durch einen synonymen Ausdruck как долго (,wie lange') ersetzt wird. Die meisten Reparaturdurchführungen - zwei im russischen und sieben im deutschen Korpus - lassen sich als Spezifizierungen betrachten (vgl. (154) für das Russische sowie (148) für das Deutsche). Darunter geht vier Reparaturdurchführungen in den deutschen Dialogen eine Reparaturinitiierung mit ich meine oder ich meinte voran. Der Reparaturoperation Erklärung im weiteren Sinne können fünf Reparaturoperationen aus dem deutschen Korpus zugeordnet werden, u.a. beispielsweise (153), wobei die Grenzen zur Spezifizierung fließend sind.

\subsubsection{Reaktionen auf aufgeklärte Missverständnisse}

Die häufigsten Reaktionen auf eine Reparaturdurchführung beim Bearbeiten eines Missverständnisses sind Erkenntnisprozessmarker im Sinne von Imo (2009) bzw. ,change-of-state‘ tokens im Sinne von Heritage (1984) (vgl. Golato/ Betz 2008). Das deutsche achso ist mit fünf Vorkommnissen in 15 Missverständnissen im next opportunity space (vgl. (152) und (153)) sowie in den beiden Missverständnissen im after next opportunity space vertreten (vgl. (147)). Darüber hinaus kommt in (157) ein anderer Erkenntnisprozessmarker, nämlich oh, vor (vgl. \#4). Im russischen Subkorpus finden sich zwei Fälle, in denen Schreiber ein behobenes Missverständnis mit dem Erkenntnisprozessmarker a (,achso`) quittieren (vgl. (149) und (154)).

Neben der Option, mit einem Erkenntnisprozessmarker seine Reinterpretation der Problemquelle zu signalisieren, sodass sich die durchgeführte Reparatur als erfolgreich (bzw. geglückt in Sinne von Egbert 2009: 70) herausstellt, kann der Rezipient auf die Ursache seiner ursprünglichen Interpretation eingehen. Eine explizite Begründung der nicht erwartbaren Antwort liegt beispielsweise in (157) (,ich hab das k überlesen“, \#4) vor, während ein weiteres beim 
Lesen aufgetretenes Problem auch in (156) (,Wer lesen kann ist klar im Vorteil!“, \#7) thematisiert wird. Eine alternative Möglichkeit, auf die z.B. die Rezipientin in (88) zurückgreift, besteht darin, dass die falsche Interpretation dem Produzenten der Problemquelle explizit vorgelegt wird und somit als Rechtfertigung für die zurückliegende nicht erwartbare Antwort des Rezipienten fungiert. In (88) wird das Missverständnis durch das Demonstrativpronomen die erzeugt (vgl. Nachricht \#14), die die Rezipientin breiter als ihre Kommunikationspartnerin fasst. Während die Produzentin der Problemquelle damit ihre Bekannten meint, bezieht die Rezipientin das Pronomen sowohl auf die Bekannten als auch auf die Eltern der Produzentin der Problemquelle, woraus eine falsche Schlussfolgerung gezogen wird, dass die Produzentin der Problemquelle alleine zu Hause Fußball schauen wird (vgl. Nachricht \#16). Nachdem dieses Missverständnis von der Produzentin der Problemquelle geklärt wurde, geht die Rezipientin auf ihre ursprüngliche Referenzzuordnung des Pronomens die ein („Ach soo =D ich dachte die wären jetzt alle weg =D“, \#19). Die explizite Versprachlichung der angenommenen Referenzzuordnung scheint in dem Fall eine gesichtsschützende Funktion $\mathrm{zu}$ haben, die mit dem modalisierenden Emoticon ,=D“ unterstützt wird.

Eine weitere Reaktion ergibt sich aus der interaktionalen Besonderheit von Missverständnissen, die darin besteht, dass der Produzent der Problemquelle den Reparaturbedarf erst an der anschließenden Rezipientenäußerung erkennt. Wird aber die Antwort des Rezipienten vom Produzenten selbst falsch verstanden, kann er ein Missverständnis bearbeiten, das es eigentlich nicht gibt. In diesem Fall kann die auf ein vermeintlich vorliegendes Missverständnis gerichtete Reparatur vom Rezipienten als überflüssig thematisiert werden. In den untersuchten Daten wurden insgesamt drei Fälle festgestellt, die eine solche Reaktion des Rezipienten aufweisen. Zwei davon wurden bereits in (148) (,Ja deshalb wollte ich umtopfen die brauchen wohl viel wasser. :) kuss“, \#5) und (150) („Ja klar, meine ich auch :D“, \#7) diskutiert. Der dritte Beleg mit der gleichen Reaktionsstrategie findet sich in (159) („Das weiß ich, ich dachte nur so

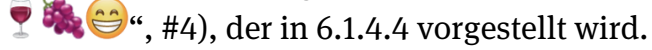

\subsubsection{Interaktionale Funktionen von aufgeklärten Missverständnissen}

Die Reparaturdurchführung in den analysierten Missverständnissen dient in erster Linie dazu, das gegenseitige Verstehen, welches aus der Perspektive des Produzenten beeinträchtigt wird (was - wie (148) uns (150) zeigen - allerdings nicht immer aus der Sicht des Rezipienten der Fall ist), wiederherzustellen (vgl. Deppermann/Schmitt 2008). In Beispiel (159) wurde die Reparaturdurchführung 
jedoch dafür benutzt, um - mehr oder weniger absichtlich - einer implizit geäußerten Bitte auszuweichen:

(159) Auszug aus dem WhatsApp-Dialog \#4248:

Schreiberin $A$

Schreiber $B$

$\rightarrow$\begin{tabular}{|c|}
\hline Stehe dir nächsten Samstag zur Hilfe \\
\hline Nachricht \#1 - 27.10.2016-13:16:05
\end{tabular}

\begin{tabular}{|c|}
\hline $\begin{array}{c}\text { Lesen nur noch diesen Samstag } \\
\text { magst }\end{array}$ \\
\hline Nachricht \#2 $\# 2$ 27.10.2016 - 13:24:05 \\
\hline
\end{tabular}

$\rightarrow$ Nee ich meine bei Nelles am 5.11. $\rightarrow$ wurde heute morgen angerufen, dachte du wüsstest davon 2 ?

Nachricht \#3 - 27.10.2016 - 13:26:05

\begin{tabular}{|c|}
\hline Das weiß ich, ich dachte nur so 0 - 9 \\
\hline Nachricht \#4 - 27.10.2016-13:33:05 \\
\hline
\end{tabular}

Direkt zum Einstieg kündigt Schreiberin $A$ an, dass sie nächsten Samstag Schreiber $B$ bei Weinlese helfen wird, wobei die Emojis stellungsfunktion (vgl. Pappert 2017: 199-202) als visuelle Kontextualisierungshinweise auf die von $A$ gemeinte Beschäftigung der Weinlese fungieren. Schreiber $B$ geht aber in \#2 auf eine andere Weinlese ein, die diesen Samstag stattfindet, und fragt subtil, ob $A$ dabei mithelfen möchte. Anstatt auf $B$ 's Bitte bzw. Angebot reagieren zu müssen, bevorzugt $A$, die vermeintlich missverstandene Referenz „[Weinlese] nächsten Samstag“ (\#1) zu bearbeiten, was ihr erlaubt, auf den zweiten von $B$ erwarteten Teil des Nachbarschaftspaars Angebot/ Bitte - Ablehnung/Akzeptanz zu verzichten. Dabei präzisiert $A$, welche Weinlese sie meint, indem sie den Namen des Weinguts („Nelles“) und das genaue Datum in \#3 liefert. B's Reaktion in \#4 zeigt allerdings, dass es eigentlich kein Missverständnis seinerseits vorlag, sondern er \#1 lediglich als Anlass benutzt hat, seine Bitte zu unterbreiten.

\subsubsection{Emoticons und Emojis bei selbstinitiierten Selbstreparaturen}

Eine Reihe von Reparaturdurchführungen bei selbstinitiierten Selbstreparaturen (inkl. Missverständnisse) werden von den Schreibern mit Emoticons, Emojis 
und vereinzelt auch mit Lachpartikeln versehen. Um feststellen zu können, ob die in Tabelle 7 präsentierte Verteilung der Emoticons und Emojis Zusammenhänge mit der Position und dem Untertyp analysierter Reparatursequenzen (selbstinitiierte Selbstreparatur im engeren Sinne vs. Missverständnis) einerseits und der Sprache der Daten andererseits aufweist, wurden zwei statistische Verfahren benutzt: der Chi-Quadrat-Test und der exakte Test nach Fisher. ${ }^{123}$

Tab. 7: Quantitative Verteilung der Emoticons und Emojis bei selbstinitiierten Selbstreparaturen im russischen und deutschen Korpus (NOS = next opportunity space, ANOS = after next opportunity space)

\begin{tabular}{lllll}
\hline & \multicolumn{2}{l}{ Russische Daten } & \multicolumn{2}{l}{ Deutsche Daten } \\
\cline { 2 - 6 } & Anzahl & $\%$ & Anzahl & $\%$ \\
Selbstinitiierte Selbstreparaturen im NOS & 7 von 38 & 18,42 & 35 von 138 & 25,36 \\
Selbstinitiierte Selbstreparaturen im same turn & 8 von 14 & 57,14 & 21 von 60 & 35,00 \\
Selbstinitiierte Selbstreparaturen im ANOS & 0 von 3 & 0,00 & 1 von 7 & 14,29 \\
Missverständnisse im NOS & 2 von 4 & 50,00 & 8 von 15 & 53,33 \\
Missverständnisse im ANOS & 0 von 0 & 0,00 & 1 von 2 & 50,00 \\
\hline
\end{tabular}

Als Erstes soll gefragt werden, ob der Gebrauch von Emojis und Emoticons in der jeweiligen Sprache in einem Zusammenhang mit der sequenziellen Position (same turn, next opportunity space und after next opportunity space) und der Art der Reparatur (selbstinitiierte Selbstreparatur im engen Sinne vs. Missverständnis) steht. Die statistischen Auswertungen zeigen, dass die fünf in Tabelle 7 für das russische Korpus aufgelisteten Gruppen von selbstinitiierten Selbstreparaturen im weiteren Sinne untereinander signifikante Unterschiede hinsichtlich des Vorkommens von Emojis und Emoticons aufweisen $\left(\chi^{2}(3)=8,8\right.$; $\mathrm{p}<0,05$; Cramer- $\mathrm{V}=0,403)$. Für das deutsche Subkorpus dagegen ließen sich keine signifikanten Unterschiede zwischen dem Gebrauch von Emoticons und Emojis und dem jeweiligen Reparaturuntertyp sowie der jeweiligen Position der Reparaturdurchführung feststellen.

In einem nächsten Schritt wurde die Verwendung von Emoticons und Emojis innerhalb jeder der fünf Gruppen von Reparaturen (vgl. Tabelle 7) für das

123 Exakte Tests nach Fisher wurden für kleine Stichproben angewandt, bei denen mindestens eine erwartete Häufigkeit unter fünf lag. Sowohl Chi-Quadrat-Tests als auch exakte Tests nach Fisher wurden in der Statistik-Software IBM SPSS Statistics (Version 27.0.1.0) durchgeführt. 
Deutsche und Russische miteinander verglichen. Wie Tabelle 7 zeigt, machen Emoticons und Emojis, die selbstinitiierte Selbstreparaturen begleiten, je nach der Gruppe von Reparaturen jeweils einen mehr oder weniger unterschiedlichen Anteil innerhalb der entsprechenden Gruppe aus. Diese Unterschiede sind jedoch statistisch gesehen nicht signifikant.

In den russischen Daten sind Emoticons und Emojis am häufigsten bei selbstinitiierten Selbstreparaturen im same turn vertreten, in 57,14\% weisen Reparaturdurchführungen in dieser Position mindestens ein Emoticon oder ein Emoji auf. Bei Missverständnissen finden sich Emoticons und Emojis in 50\% der Fälle, wohingegen selbstinitiierte Selbstreparaturen im next opportunity space diese lediglich in 18,42\% der Belege aufweisen. Etwas anders sieht die Distribution von Emoticons und Emojis in den deutschen Kurznachrichten aus. Der größte Anteil an Emoticons und Emojis lässt sich nicht bei Reparaturen im same turn, sondern bei Missverständnissen im next opportunity space $(53,33 \%)$ und after next opportunity space (50\%) beobachten, wobei sowohl im deutschen als auch im russischen Subkorpus der Anteil von Emoticons und Emojis bei Missverständnissen relativ hoch ist ( $\geq 50 \%$ ). Diese Tendenz könnte damit erklärt werden, dass Emoticons und Emojis in dem Fall eine modalisierende Funktion zu übernehmen scheinen (vgl. Imo 2015b: 150-152; Pappert 2017: 195-196) und einen Beitrag zum face work leisten. Dabei kann sich die gesichtsschützende Funktion von Emoticons und Emojis je nach Kontext zum einen auf den Rezipienten der Problemquelle richten, dessen Interpretation der problematischen Nachricht vom Produzenten als nicht zutreffend zurückgewiesen wird, was für den Rezipienten potenziell gesichtsbedrohend ist. Zum anderen kann der Produzent der Problemquelle durch den Einsatz von Emoticons und Emojis auch das eigene Gesicht wahren, da er den problematischen Charakter seiner Nachricht nicht rechtzeitig erkannt und die Problemquelle weder im Rahmen einer Revision noch im Laufe einer selbstinitiierten Selbstreparatur im next opportunity space (vgl. Kap. 6.1.1) bearbeitet hat, sondern den Reparaturbedarf erst im Nachhinein erkannt hat, nachdem ein Missverständnis bereits aufgetreten ist.

Ein weiterer - jedoch, wie bereits erwähnt, nicht signifikanter - Unterschied in Bezug auf die Verteilung von Emoticons und Emojis in den deutschen und russischen Daten besteht darin, dass im deutschen Korpus ein Emoji bei selbstinitiierten Selbstreparaturen im after next opportunity space identifiziert wurde. Dabei handelt es sich um das iterierte Emoji aus Nachricht \#17 in (122c), das in einer eigenständigen Nachricht zwischen der Nachricht mit dem Reparandum und derjenigen mit der Reparaturdurchführung realisiert wurde. Durch seine Position schließt sich das Emoji zum einen an das Reparandum an und modalisiert dieses, indem es \#16 als scherzhaft gemeinte Äußerung mar- 
kiert. Zum anderen kann aber das Emoji dank seiner Grenzposition auch als eine Art Ankündigung der bevorstehenden Reparaturdurchführung angesehen werden, welche in der nächsten Nachricht erfolgt.

Allerdings muss darauf hingewiesen werden, dass bei vielen in Tabelle 7 aufgelisteten Gruppen von selbstinitiierten Selbstreparaturen die Anzahl der im Korpus identifizierten Reparaturen relativ gering ist, sodass die Verteilung sowie der Anteil von Emoticons und Emojis innerhalb der jeweiligen Gruppe nur unter Vorbehalt ausgewertet werden kann.

Wenn man die in den beiden Teilkorpora vorliegenden Emoticons und Emojis aus formaler Perspektive betrachtet, stellt man fest, dass die deutschen Daten im Vergleich zu den russischen eine breitere Palette an Emoticons und Emojis aufweisen. Während sich im russischen Korpus hauptsächlich das Emoticon :-) und seine Varianten ), :), =) (14 Fälle) und lediglich drei Emojis ( 3 , ¿ und ein unbekanntes Emoji, das bei der Einstellung in die Datenbank nicht erkannt wurde) verwendet werden, verteilen sich 66 Tokens im deutschen Subkorpus auf zehn Types von Emoticons und acht Types von Emojis. Dabei machen sechs Emoticons :- $D,: D,:-)$, :), ;-) sowie ;) mit 36 Fällen mehr als die Hälfte aller Verwendungen von Emoticons und Emojis bei Reparaturen aus. Neben Emoticons werden in den deutschen Kurznachrichten 14 Emojis verwendet, darunter auch $\Leftrightarrow,=0$, And $\theta$.

Aus funktionaler Perspektive stehen bei selbstinitiierten Selbstreparaturen zwei Funktionen von Emoticons und Emojis im Vordergrund, und zwar die modalisierende und kommentierende (vgl. Pappert 2017: 195-198). Die kommentierende Funktion erfüllen in $(122 \mathrm{a}-\mathrm{b})$ beispielsweise das Emoji $\%$ (\#4) sowie das Emoticon -.- (\#8), mit denen die Schreiberin ihre Verärgerung über Fehler bzw. missglückte Reparaturdurchführung zum Ausdruck bringt. Eine modalisierende Funktion kann dagegen beispielsweise den Emoticons zugeschrieben werden, die eine Frotzelaktivität konstituierende Reparaturen begleiten (vgl. ;) in \#3 aus (132)) oder den Wechsel der Interaktionsmodalität markieren (vgl. ;) in \#3 aus (123)). Des Weiteren können Emojis aber auch beispielsweise eine Darstellungsfunktion ausüben (vgl. Pappert 2017: 199-202), wie das Emoji aus (159), das in dem Kontext auf die Weinlese Bezug nehmen soll. 


\subsubsection{Selbstinitiierte Selbstreparaturen im Kontrast}

\subsubsection{Selbstinitiierte Selbstreparaturen in der gesprochenen Sprache und in der Kurznachrichtenkommunikation}

Der durch die Analyse in 6.1.1-6.1.5 gewonnene Einblick in die Mechanismen der selbstinitiierten Selbstreparaturen in der Kurznachrichtenkommunikation lässt darauf schließen, dass diese zwar gewisse Überschneidungen mit denjenigen aus der gesprochen Sprache aufweisen, gleichzeitig aber über eine Reihe unikaler Besonderheiten verfügen. Die vorliegende Untersuchung zeigt beispielsweise, dass in der Kurznachrichtenkommunikation genau wie in der gesprochenen Sprache neben selbstinitiierten Selbstreparaturen im engeren Sinne (vgl. Kap. 6.1.1-6.1.3) auch Missverständnisse, also third position repairs (vgl. Kap. 6.1.4), vorkommen, wobei deren sequenzielle Positionen in Anbetracht der kommunikativen Bedingungen in der interaktionalen schriftlichen Kommunikation anders als in der gesprochenen Sprache konzeptualisiert werden müssen (vgl. Kap. 3.3). In Bezug auf die drei grundlegenden Komponenten einer Reparatursequenz - das Reparandum, die Reparaturinitiierung und die Reparaturdurchführung - lassen sich ebenso einige Gemeinsamkeiten zwischen Reparaturen in der medialen Mündlichkeit und Schriftlichkeit beobachten. Bei Reparanda trifft das zum einen v.a. auf lexiko-semantische und pragmatische Reparanda bei selbstinitiierten Selbstreparaturen im same turn sowie next und after next opportunity space zu. Zum anderen eignen sich die Kategorien, die Schegloff (1992) für Problemtypen bei Missverständnissen in der gesprochenen Sprache entwickelt hat, nämlich die problematische Referenz und die problematische sequenzielle Implikation, auch für die Analyse von Reparaturen in der computervermittelten Kommunikation. Im Bereich der Reparaturinitiierungen lassen sich ebenso einzelne Parallelen $\mathrm{zu}$ Reparaturen in der mündlichen Kommunikation erkennen: Selbstinitiierte Selbstreparaturen in den untersuchten Daten werden mit Partikeln wie ach, ehm oder $H y$ (,na ja, halt, nun') sowie phrasalen und satzwertigen Reparaturmarkern wie ich meine oder в смысле (,im Sinne von') eingeleitet. Das Gleiche gilt auch für Reparaturdurchführungen, in deren Rahmen Schreiber z.B. auf in der gesprochenen Sprache belegte Reparaturoperationen wie Substitutionen und Insertionen bzw. - im Falle von Missverständnissen - auf Spezifizierungen und Umformulierungen zurückgreifen.

Allerdings fällt auf, dass, abgesehen von einigen Gemeinsamkeiten, Reparaturen in der Kurznachrichtenkommunikation sich von denen in der gesprochenen Sprache in einigen Aspekten unterscheiden. Diese Unterschiede hängen u.a. mit folgenden Faktoren zusammen, die z.T. miteinander eng verbunden 
sind und veranschaulichen, wie Schreiber mit verschiedenen, durch das Kommunikationsmedium und die Kommunikationstechnologie sowie durch das jeweilige Sprachsystem/Schriftsystem geschaffenen Affordanzen (vgl. Hutchby 2001; Zillien 2009 und Marx/Schmidt 2019) umgehen:

(i) mit der medialen Realisierung (vgl. Koch/Oesterreicher 1985) der drei Komponenten einer Reparatur (Stichwörter: mediale Mündlichkeit und mediale Schriftlichkeit);

(ii) mit der technischen Seite des eingesetzten Kommunikationsmediums im Sinne von Dürscheid (2005);

(iii) mit den Kommunikationsbedingungen an sich, d.h. mit der zeitlichen und räumlichen Nähe bzw. Distanz, welche letztendlich Auswirkungen auf den Grad der Synchronizität der Kommunikation ausüben (vgl. Koch/Oesterreicher 1985; Dürscheid 2005);

(iv) mit Besonderheiten der jeweiligen Kommunikationsform (SMS vs. WhatsApp, Viberund iMessage).

(i) Die medial schriftliche Realisierung der analysierten Belege mit selbstinitiierten Selbstreparaturen wirkt sich in erster Linie auf Reparanda und Reparaturinitiierungen aus. Während in der gesprochenen Sprache solche Problemquellen bearbeitet werden müssen, die auf der lautlichen Ebene angesiedelt sind (vgl. (6)), treten an deren Stelle in der medial schriftlichen Kurznachrichtenkommunikation (ortho)graphische Reparanda (vgl. Kap. 6.1.1.1). Dabei betreffen (ortho)graphische Reparaturen, wie in Kap. 6.1.1.1 diskutiert wurde, nicht nur die Schreibung einzelner Lexeme, sondern auch Interpunktionszeichen, Emoticons und sogar die Groß- und Kleinschreibung.

Bei Reparaturinitiierungen wird die mediale Schriftlichkeit einerseits dafür genutzt, um neue Initiierungsstrategien $\mathrm{zu}$ entwickeln, und andererseits, um die bereits aus der gesprochenen Sprache bekannten Strategien kompakter darzustellen. Im ersten Fall handelt es sich um nicht-alphabetische Reparaturmarker wie Asteriske oder Schrägstriche (vgl. Kap. 6.1.1.2) sowie um solche graphischen Ressourcen, die zwar nicht unbedingt als Reparaturmarker im engeren Sinne aufgefasst werden können, trotzdem aber einen Beitrag zur Gestaltung einer Reparatur leisten, wie Klammern, Auslassungspunkte oder die Majuskelschreibung (vgl. Kap. 6.1.2.2). Im zweiten Fall werden zwei Reparaturmarker aus dem russischen Subkorpus gemeint, die in den analysierten Daten nicht in deren vollständigen Form, sondern als eine schriftsprachlich konventionalisierte Abkürzung T.e. (,d.h.'; vgl. (110)) sowie als eine kreative Verschmelzung einer Präposition und eines nicht-standardsprachlich abgekürzten Nomens всм (für в смысле ,im Sinne von'; vgl. Kap. 6.1.3) vorliegen. 
(ii) Das Kommunikationsmedium übt in den untersuchten Kurznachrichten v.a. auf die Texteingabe Einfluss aus, was sich v.a. in den nicht erwünschten Eingriffen des aktivierten Autokorrekturprogramms äußert. Ein extremer Fall liegt in $(122 \mathrm{a}-\mathrm{d})$ vor, in dem durch die Autokorrektur innerhalb von dreizehn Nachrichten einer Schreiberin insgesamt sechs (ortho)graphische Reparanda erzeugt werden.

Dadurch, dass Messenger-Dienste inzwischen nicht nur auf Smartphones, sondern auf anderen Geräten, u.a. auch auf PCs und Laptops, installiert werden können, wird das Entstehen solcher (ortho)graphischer Reparanda wie in (89) ermöglicht, die eigentlich dadurch zustande kommen, dass Schreiber auf die von ihnen ausgewählte Tastatursprache nicht achten und anstatt eines Textes in einer Sprache eine sinnlose Kombination aus Zeichen einer anderen Sprache eingeben.

(iii) Bei den für Reparaturen relevanten Kommunikationsbedingungen in der Kurznachrichtenkommunikation spielt das sogenannte „Mühlen-Prinzip“ im Sinne von (Wichter 1991) eine entscheidende Rolle (vgl. auch Storrer 2001; Beißwenger 2010). Dieses wirkt sich v.a. auf die sequenzielle Organisation von selbstinitiierten Selbstreparaturen aus (vgl. Kap. 3.3), und zwar in der Hinsicht, dass bei der Bestimmung der Position der Reparaturdurchführung in Bezug auf die Nachricht mit der Problemquelle nicht auf deren tatsächliche Position im kompletten Nachrichtenverlauf, sondern auf deren Position unter Nachrichten des Produzenten der Problemquelle geachtet wird. Dabei bleiben also Nachrichten des Rezipienten der Problemquelle unberücksichtigt, deren Verfassen und Abschicken der Produzent der Problemquelle, während er sich mit der Bearbeitung des Reparandums befasst, nicht verhindern kann. Ein solches Umdenken der für die gesprochene Sprache geltenden sequenziellen Positionen erfordert selbstverständlich die Herausarbeitung neuer Kategorien für die Bezeichnung sequenzieller Position der Reparaturen in der medialen Schriftlichkeit, wie z.B. die in der vorliegenden Arbeit verwendeten Begriffe same turn, next und after next opportunity space (vgl. Schönfeldt/Golato 2003). Der Forschungsstand zu dieser Frage wurde bereits in Kap. 3.3 vorgestellt.

Im Zusammenhang mit selbstinitiierten Selbstreparaturen im same turn muss hervorgehoben werden, dass diese in den analysierten Daten meist eine rhetorisch-stilistische Funktion erfüllen. Dadurch, dass die Problemquelle und die Reparaturdurchführung in der gleichen Nachricht platziert werden, wird deutlich, dass der Produzent der Problemquelle diese bereits vor dem Abschicken der Nachricht erkannt hat und daher sie potenziell im Rahmen einer Revision, also unsichtbar für den Rezipienten, hätte bearbeiten können. Des Weiteren zeichnet sich jeweils eine selbstinitiierte Selbstreparatur im same turn aus 
dem deutschen und dem russischen Subkorpus dadurch aus, dass sie als Ressource für eine inszenierte prospektive Reparatur fungiert (vgl. (131)). Mit Ausnahme dieser zwei Fälle stellen alle anderen selbstinitiierten Selbstreparaturen retrospektive Reparaturen dar, d.h. es wird ein zurückliegendes Reparandum bearbeitet (in den Daten von Pfeiffer (2015: 53, 91) dagegen treten prospektive Reparaturen in 50,3\% der gesamten Anzahl von Reparaturen auf, während retrospektive Reparaturen nur in 25,7\% der Fälle belegt sind). Eine solche quantitative Verteilung der prospektiven und retrospektiven Reparaturen in den untersuchten Kurznachrichten lässt sich damit erklären, dass der Rezipient in der schriftlichen interaktionalen Kommunikation mit fertigen Schreibprodukten konfrontiert wird, wohingegen der Formulierungsprozess - und somit auch die Wahrnehmung prospektiver Reparaturen - in der gesprochenen Sprache dem Rezipienten unmittelbar zugänglich ist. In der interaktionalen Schriftlichkeit besteht jedoch die Möglichkeit, v.a. prospektive, aber auch retrospektive, selbstinitiierte Selbstreparaturen während der Produktion einer Nachricht im Rahmen von Revisionen zu bearbeiten, sodass diese für den Rezipienten im Dunkeln bleiben (zu Revisionen vgl. Beißwenger 2010).

(iv) Für die Auseinandersetzung mit dem letzten in diesem Kapitel angesprochenen Faktor, der die Besonderheiten der Textnachrichten bei der SMS-Kommunikation und der Kommunikation via WhatsApp, Viber und iMessage betrifft, ist das dieser Arbeit zugrundeliegende Korpus leider nicht optimal geeignet. Dennoch kann an dieser Stelle eine Frage aufgeworfen werden, die bereits in 6.1.1.1 angesprochen wurde und als Forschungsdesiderat für weitere empirischen Studien formuliert werden kann, nämlich, ob und inwiefern einzelne Funktionen von Messenger-Diensten, die aber in der SMS-Kommunikation nicht gegeben sind, bei selbstinitiierten Selbstreparaturen eingesetzt werden. Das betrifft in erster Linie die Möglichkeit, eine bereits verschickte Nachricht zu löschen (vgl. (100)). Eine weitere technische Funktion, die in der vorliegenden Arbeit in Bezug auf fremdinitiierte Selbstreparaturen diskutiert wird (vgl. Kap. 6.2.1.6) und die auch potenziell für selbstinitiierte Selbstreparaturen in Frage käme, besteht darin, dass in den Messenger-Apps eine zurückliegende Nachricht ähnlich wie in der Foren-Kommunikation als Zitat in die emergente Nachricht integriert werden kann. Dadurch könnten die ursprüngliche Nachricht und die Reparaturdurchführung zueinander in Verbindung gebracht werden, sodass das Erkennen des Reparandums - v.a. wenn die beiden Nachrichten mehrere Nachrichten auseinanderliegen - gewährleistet wird, ohne dass dieses vom Schreiber erneut eingetippt werden muss. 


\subsubsection{Selbstinitiierte Selbstreparaturen im Russischen und Deutschen}

Aus sprachkontrastiver Perspektive konnten trotz der unausgewogenen Größenverhältnisse des russischen und des deutschen Subkorpus dennoch einige erste Erkenntnisse über systematische Gemeinsamkeiten und Unterschiede bei Reparaturen in den beiden Sprachen gewonnen werden. Dabei wurden die in dem jeweiligen Korpus auftretenden selbstinitiierten Selbstreparaturen v.a. qualitativ miteinander verglichen, sodass ein systematischer quantitativer Vergleich u.a. auch in Bezug auf die Verteilung einzelner Initiierungsformate oder in Bezug auf Häufigkeiten bestimmter Reparanda in jeder einzelnen Sprache - ein Forschungsdesiderat für Folgeuntersuchungen anhand größerer Korpora bleibt.

In den Folgestudien könnte beispielsweise näher auf den Einsatz graphischer Reparaturmarker in den beiden Sprachen eingegangen werden, darunter auch auf die Frage, ob Schrägstriche (vgl. (104) und (105)) bei Reparaturinitiierungen in den russischen Daten als idiosynkratisch zu werten sind oder ob diese bereits in der russischen - und evtl. auch in der deutschen - Kurznachrichtenkommunikation als konventionalisierte Reparaturmarker fungieren. Des Weiteren kann im Bereich der Reparaturinitiierungen für die russischen Daten die Verwendung von Partikeln als Reparaturmarker anhand einer größeren Datenkollektion erforscht werden, um festzustellen, welche Partikeln außer $н y$ (,na ja, halt, nun') von Schreibern für Reparaturzwecke eingesetzt werden. Darüber hinaus können die in den analysierten Daten belegten phrasalen und satzwertigen Reparaturmarker ich meine für das Deutsche und в смысле (,im Sinne von') für das Russische in den Blick genommen werden, um herauszufinden, ob deren wortwörtliche Äquivalente aus der jeweils anderen Sprache - я имею в виду (,ich meine') und im Sinne von - ebenso als Reparaturmarker verwendet werden können.

Einige Anknüpfungspunkte für weitere Forschungen bieten auch die Reparaturdurchführungen an. In den untersuchten Daten finden sich z.B. zwei Belege, in denen nicht als utterance deflater (Haiman 1995: 331) fungiert und somit zur Konstitution einer pragmatischen Reparatur beiträgt (vgl. (132) und (133)). Es kann also der Frage nachgegangen werden, ob die russische Negationspartikel не (,nicht') oder das Responsiv нет (,nein`) ebenso in einer vergleichbaren Funktion auftreten können.

Darüber hinaus lohnt sich ein sprachkontrastiver Vergleich im Bereich des Gebrauchs von Emoticons und Emojis in der jeweiligen Sprache. Die hier analysierten Daten haben beispielsweise gezeigt, dass selbstinitiierte Selbstreparaturen im Russischen im Gegensatz zu denen im Deutschen eine geringe Varianz an Emoticons und Emojis aufweisen. Diese Tendenz soll aber in einem ausgewogenen Korpus überprüft werden. 


\subsection{Fremdinitiierte Selbstreparaturen in der Kurznachrichtenkommunikation}

Das deutsche Korpus weist beinahe genauso viele fremdinitiierte wie selbstinitiierte Selbstreparaturen auf. Während sich die Anzahl von selbstinitiierten Selbstreparaturen auf 223 Belege beläuft, kommen in den deutschen Daten 222 fremdinitiierte Selbstreparaturen vor. In den russischen Kurznachrichten ist der Unterschied bei der quantitativen Verteilung der beiden Typen von Selbstreparaturen etwas größer: 73 fremdinitiierte gegenüber 59 selbstinitiierten Selbstreparaturen. Dabei muss darauf hingewiesen werden, dass manche der in den untersuchten Daten gesichteten Reparatursequenzen zwar die Problemquelle sowie die Reparaturinitiierung, aber keine Reparaturdurchführung enthalten. Dafür sind unterschiedliche Gründe verantwortlich, welche in Kap. 6.2.3 näher diskutiert werden. An dieser Stelle soll zunächst lediglich angemerkt werden, dass Reparanda in 206 von 222 Fällen aus den deutschen Verläufen und in 67 von 73 fremdinitiierten Selbstreparaturen in den in der MoCoDa zur Verfügung gestellten russischen Dialogen bearbeitet werden.

Für Reparatursequenzen, in denen sowohl Problemquellen als auch Reparaturdurchführungen in den analysierten Daten vorlagen, wurden in Anlehnung an die in Kapitel 3.3 präsentierte Typologie Reparaturpositionen ermittelt. Die Analyse der Reparaturpositionen hat ergeben, dass über 80\% fremdinitiierter Selbstreparaturen im jeweiligen Korpus im next opportunity space, also in der nächsten Nachricht des Produzenten der Problemquelle nach der Nachricht mit dem Reparandum, erfolgen (172 Reparaturen im deutschen und 58 im russischen Teilkorpus). Gleichzeitig liegen in den deutschen Daten 12\% $(n=25)$ und in den russischen 9\% $(\mathrm{n}=6)$ fremdinitiierter Selbstreparaturen im after next opportunity space vor, d.h. zwischen der Nachricht mit der Problemquelle und der Nachricht mit der Reparaturdurchführung befindet sich mindestens eine weitere vom Produzenten der Problemquelle verschickte Nachricht. Wenn man sich fremdinitiierte Selbstreparaturen im after next opportunity space im Detail anschaut, stellt man fest, dass sich diese Position der Reparaturdurchführung in vier Kontexten finden lässt. Dabei erscheinen weitere Nachrichten des Produzenten der Problemquelle im jeweiligen Nachrichtenverlauf (i) nach der Nachricht mit dem Reparandum, aber vor der Nachricht mit der Fremdinitiierung ( $\mathrm{n}=19$ in den deutschen und $\mathrm{n}=4$ in den russischen Daten), (ii) erst nach der Nachricht mit der Fremdinitiierung, aber vor derjenigen mit der Reparaturdurchführung ( $\mathrm{n}=5$ in den deutschen und $\mathrm{n}=1$ in den russischen Daten) oder (iii) an den beiden genannten Stellen gleichzeitig, (i) und (ii) werden also kombiniert ( $\mathrm{n}=1$ in den deutschen und $\mathrm{n}=1$ in den russischen Daten). Hinsichtlich der 
Reparaturposition gibt es allerdings noch eine weitere Option, bei der es sich um die auf zwei Nachrichten verteilte Reparaturdurchführung handelt, sodass sich die erste Nachricht, die den ersten Teil der Reparaturdurchführung enthält, im next opportunity space und die nächste streng genommen im after next opportunity space befindet. Es liegt also das Splitting im Sinne von Beißwenger (2007) vor ( $n=8$ in den deutschen und $n=3$ in den russischen Daten). Die hier skizzierten sequenziellen Umgebungen für fremdinitiierte Selbstreparaturen im after next opportunity space legen offen, dass ,aufgrund der NichtSimultaneität von Produktion und Rezeption“, die „die Koordination von Sprachhandlungen in nicht unerheblicher Weise erschwert und überdies zu einer nie gänzlich synchronen Sicht der Beteiligten auf den aktuellen Progress gemeinsam gewebter Handlungssequenzen führt“ (Beißwenger 2005: 64), es gelegentlich dazu kommt, dass die Reparaturinitiierung erst dann geliefert wird, nachdem die Problemquelle bereits einige Nachrichten zurückliegt, gefolgt von einer oder mehreren weiteren Nachrichten des Produzenten der Problemquelle. Es ist dennoch erstaunlich, dass, sobald die Fremdinitiierung geliefert wurde, sie jeweils - in mehr als $97 \%$ der Fälle - in der nächsten im Nachrichtenverlauf platzierten Nachricht des Produzenten der Problemquelle bearbeitet wird. Lediglich in sechs Belegen im deutschen und zwei Beispielen im russischen Korpus liegen zwischen der Nachricht mit der Fremdinitiierung und der Reparaturdurchführung andere Nachrichten des Produzenten der Problemquelle. Diese acht Fälle scheinen allerdings in gewisser Weise mit der Richtung zusammenzuhängen, in der der Produzent der Problemquellee auf die Nachrichten seines Kommunikationspartners bzw. deren Teile reagiert (diese können vom Produzenten der Problemquelle z.B. von oben nach unten ,abgearbeitet werden, wie in Nachrichten \#5-6 in (104); zur Flächigkeit vgl. Beißwenger/Pappert 2019: 42, 62). Eine solche ,Verschiebung‘ der Reparaturdurchführung zugunsten einer anderen sprachlichen Handlung kann zudem vereinzelt als ein Indiz dafür interpretiert werden, dass diese Handlung implizit mehr oder weniger intendiert als eine für die laufende Interaktion wichtigere eingestuft und daher ,vorgezogen“ wird (vgl. Nachrichten \#6 und \#7 in (182)).

Abgesehen von der oben beschriebenen Tendenz, die Reparaturdurchführung möglichst nah am Reparandum oder zumindest an der Reparaturinitiierung zu platzieren und somit die gegenseitige Verständigung so schnell wie möglich wiederherzustellen (für die gesprochene Sprache vgl. Schegloff 1979, 2007; Heritage 2007), zeichnet sich auch bei der Position der Fremdinitiierung eine ähnliche Tendenz ab, nämlich, das Vorhandensein eines Problems möglichst unmittelbar nach der Nachricht mit der Problemquelle zu signalisieren. Lediglich in weniger als 10\% der im jeweiligen Korpus vorhandenen Fremdiniti- 
ierungen erscheint zwischen der Nachricht mit dem Reparandum und der Nachricht mit der Reparaturinitiierung eine weitere Nachricht des Rezipienten der Problemquelle ( $\mathrm{n}=16$ in den deutschen und $\mathrm{n}=6$ in den russischen Dialogen; vgl. Nachrichten \#53-54 aus dem WhatsApp-Dialog \#4353 in der MoCoDa). Bei der Realisierung einer Fremdinitiierung scheinen Rezipienten der Problemquelle also dazu zu neigen, ihre Kommunikationspartner möglichst zeitnah auf das aus ihrer Sicht vorliegende Problem aufmerksam zu machen und ihnen die Möglichkeit zu geben, dieses zu beheben. Eine räumlich relativ dicht an dem Reparandum gelieferte Fremdinitiierung erhöht außerdem die Chancen, dass sie vom Produzenten der Problemquelle als solche erfolgreich erkannt und eingelöst wird.

Da bei der Analyse keine ausschlaggebenden Unterschiede zwischen fremdinitiierten Selbstreparaturen im next und after next opportunity space festgestellt werden konnten, werden diese im Folgenden nicht separat voneinander betrachtet, sondern es wird auf die in den untersuchten Daten auftretenden Fremdinitiierungsformate, Typen von Reparanda sowie Reparaturdurchführungen für fremdinitiierte Selbstreparaturen in den beiden Positionen zusammen eingegangen. ${ }^{124}$ Darüber hinaus wird auch die Reaktion auf fremdinitiierte Selbstreparaturen sowie der Beitrag von Emoticons und Emojis bei diesem Reparaturtyp kurz vorgestellt. Der sich daran anschließenden Auseinandersetzung mit den interaktionalen Funktionen von selbstinitiierten Fremdreparaturen im next und after next opportunity space folgt eine Zusammenfassung, in der die im vorliegenden Kapitel präsentierten Analyseergebnisse die Grundlage dafür bilden, um fremdinitiierte Selbstreparaturen einerseits in der interaktionalen Mündlichkeit und Schriftlichkeit und andererseits in der deutschen und russischen Kurznachrichtenkommunikation einander gegenüberzustellen und die sich dabei ergebenden Unterschiede und Gemeinsamkeiten zu diskutieren.

\subsubsection{Fremdinitiierungen}

\subsubsection{Unspezifische Fremdinitiierungen}

Wie bereits in Kapitel 2.3.1.1 erwähnt, signalisieren unspezifische Reparaturinitiierungen lediglich ein vorliegendes Verstehensproblem, ohne dieses jedoch weiter zu spezifizieren (vgl. Drew 1997; Egbert 2009; Baranova 2015; Kendrick 2015). In dieser Funktion werden in der gesprochenen Sprache v.a. Partikeln

124 Vgl. Fußnote 30. 
bzw. Interjektionen, unspezifische Fragewörter und Höflichkeitsfloskeln benutzt (vgl. Egbert 2009; Baranova 2015; Dingemanse/Enfield 2015).

In den analysierten Kurznachrichtendaten kommen ausschließlich Interjektionen sowie unspezifische Fragewörter vor, wobei es einen signifikanten Unterschied zwischen unspezifischen Fremdinitiierungen in den beiden Korpora gibt $\left(\chi^{2}(1)=5,2 ; p<0,05 ;\right.$ Cramer-V=0,133). Während im russischen Subkorpus lediglich ein einziger Fall $\left(1,2 \%{ }^{125}\right)$ mit einem unspezifischen Fragewort zu finden ist, weist das deutsche Subkorpus 21 unspezifische Fremdinitiierungen $(7,7 \%)$ auf, die sowohl in Form der Interjektion $h a ̈$ (acht Tokens, inkl. der Variante $h \ddot{)}$ ) als auch in Form des unspezifischen Fragepronomens was (13 Tokens, inkl. der regionale Variante wat $(t)^{126}$ sowie what ${ }^{127}$ ) realisiert sind. Das Nicht-Vorkommen der Interjektionen $M\left(, \mathrm{hm}^{*}\right)$ und $a($,hä') im russischen Subkorpus könnte damit zu tun haben, dass diese Interjektionen im Gegensatz zu ihren deutschen Pendants möglicherweise nur das Vorhandensein von akustischen Verstehensproblemen signalisieren und dementsprechend in der schriftbasierten informellen Kommunikation keinen Einsatz finden. Um diese These zu stützen oder zu widerlegen, benötigt man aber eine systematische Studie zum Gebrauch von Interjektionen $M\left(, \mathrm{hm}^{\circ}\right)$ und $a$ (,hä') bei Reparaturen in der russischen gesprochenen Sprache, die die in solchen Kontexten auftretenden Prob-

125 Die Prozentangaben in Klammern zeigen im gesamten Kapitel 6.2 den Anteil des jeweiligen Initiierungsformats an allen Fremdinitiierungen im entsprechenden Subkorpus. Dabei muss allerdings beachtet werden, dass zehn fremdinitiierte Selbstreparaturen in den russischen und 37 Reparatursequenzen in den deutschen Daten je zwei Fremdinitiierungen aufweisen. Des Weiteren liegen in einem russischen und sieben deutschen Belegen jeweils drei Reparaturinitiierungen pro Reparatur vor. Daraus folgt, dass 73 fremdinitiierte Selbstreparaturen aus dem russischen Teilkorpus 85 Fremdinitiierungen enthalten, während sich die Anzahl der Reparaturinitiierungen im deutschen Teilkorpus mit 222 fremdinitiierten Selbstreparaturen auf 271 Fremdinitiierungen beläuft.

126 Die meisten Daten aus der Datenbank MoCoDa wurden an der Universität Duisburg-Essen gesammelt, was das Vorkommen dieser u.a. für niederdeutsche Dialekte typischen Variante erklärt (vgl. auch die Verteilung der Varianten was und wat im Atlas der deutschen Alltagssprache unter http://www.atlas-alltagssprache.de/runde-1/f17a-c/).

127 Bei what (zwei Tokens) könnte es sich sowohl um eine intendiert eingesetzte englischsprachige Form als auch um ein durch die Autokorrektur abgeändertes Wort handeln. Im ersten Fall reichen die Daten allerdings nicht, um zu diskutieren, wodurch der Gebrauch von what ausgelöst werden könnte, da in den dazugehörigen Metadaten kein Hinweis auf den Stand der Englischkenntnisse der Schreiber gegeben wird. Außerdem weist keiner der beiden Dialoge - bzw. der in der Datenbank MoCoDa abgebildeten Dialogabschnitte (vgl. ,endloser Dialog، bei Imo 2015a) - ein Code-Switching ins Englische auf. Daher muss die Frage, inwiefern what intendiert gebraucht wird und - falls ja - ob es funktionale Unterschiede zwischen what und was gibt, in weiteren Untersuchungen geklärt werden. 
lemquellen ermitteln soll. Alternativ kann das Ausbleiben der Interjektionen als Reparaturressourcen in den russischen Kurznachrichten darauf zurückgehen, dass solche Fälle in dem relativ überschaubaren russischsprachigen Analysekorpus einfach nicht miterfasst worden sind. Im Gegensatz dazu lässt sich das Fehlen der Höflichkeitsfloskeln bei Fremdinitiierungen in beiden Korpora besser erklären, wenn man diese Tatsache mit den Ergebnissen von Selting (1987a), Egbert (1996), Dingemanse/Blythe/Dirksmeyer (2014) sowie Baranova (2015) in Relation setzt, die zeigen, dass Höflichkeitsfloskeln meistens in formellen Kommunikationssituationen verwendet werden und/oder mit nicht-verbalen Komponenten der Kommunikation, wie z.B. mit dem Blickverhalten, gekoppelt sind, sodass sich deren Vorkommen in einem informellen Interaktionssetting als eher wenig wahrscheinlich erweist.

\section{Unspezifische Fragewörter}

In der mündlichen Kommunikation kann mithilfe einer unspezifischen Fremdinitiierung eine breite Palette an Problemquellen thematisiert werden (vgl. Kap. 2.3.1.1). In den analysierten Daten lässt sich eine ähnliche Tendenz nachweisen. Dabei lassen sich allerdings einige Unterschiede bei den angezeigten Problemtypen feststellen. Während in der gesprochenen Sprache unspezifische Reparaturinitiierungen oft einen Hinweis auf das vorliegende Hörverstehensproblem geben, werden unspezifische Fremdinitiierungen im Analysekorpus nicht für Rezeptionsprobleme (sei es diejenige des Lesens oder - im Falle von Sprachnachrichten - des Hörens), sondern für Bedeutungsverstehens- sowie Erwartungsprobleme eingesetzt:

(160) Auszug aus dem WhatsApp-Dialog \#3059:

Schreiber $A$

Schreiber $B$

\begin{tabular}{|c|}
\hline ich klemm mir heute abend einen ;) \\
\hline Nachricht \#21 - 13.02.2014 - 14:05:05 ${ }^{128}$ \\
\hline
\end{tabular}

\begin{tabular}{|c|}
\hline hat man sich ja auch ma verdient, woll.... \\
\hline Nachricht \#22 - 13.02.2014 - 14:05:05 \\
\hline
\end{tabular}

\begin{tabular}{|c|}
\hline isso \\
\hline Nachricht \#23 $-13.02 .2014-14: 05: 05$ \\
\hline
\end{tabular}

128 Vgl. Fußnote 117. 


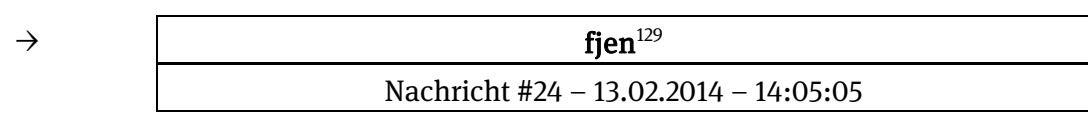

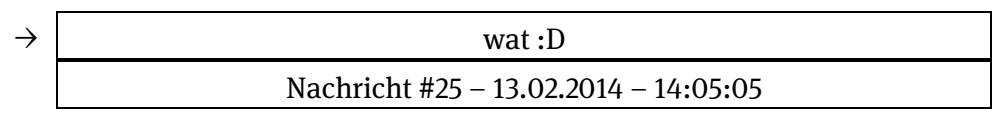

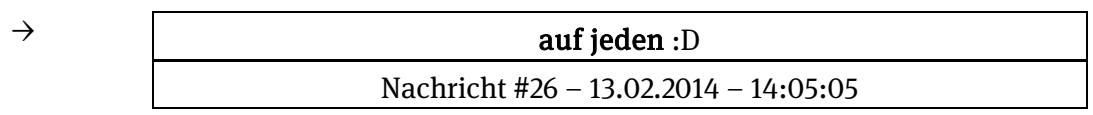

\begin{tabular}{|c|}
\hline haha \\
\hline Nachricht \#27 - 13.02.2014 - 14:05:05 \\
\hline
\end{tabular}

In Auszug (160) planen zwei Freunde einen gemeinsamen Abend und besprechen einige Details. Die Problemquelle „fjen“ (\#24), die die regionale Variante der Präpositionalphrase auf jeden Fall repräsentieren soll und vermutlich durch ebenfalls regional geprägtes „,isso“ in \#23 ausgelöst wurde, wird von $A$ in \#25 hinterfragt. Dabei bleibt $A$ bei dialektalen Varianten und benutzt das für das Niederdeutsche typische Fragepronomen „wat“ in Kombination mit einem Emoticon, das ,lautes Lachen` symbolisiert. Es kann allerdings nicht geklärt werden, ob $A$ 's Fremdinitiierung ernst gemeint oder nur als Sprachspiel gedacht wurde. In jedem Fall führt diese Fremdinitiierung dazu, dass $B$ das bestehende Problem bearbeitet, indem er das Reparans „auf jeden“ in Kombination mit demselben Emoticon wie in der Initiierungsnachricht von $A$ verschickt. Die erfolgreiche Lösung des Problems wird durch $A$ 's ,ikonisches Lachen` (vgl. auch Imo 2015b) in \#27 implizit bestätigt.

Mit unspezifischen Fremdinitiierungen können auch Erwartungsprobleme aufgezeigt werden. Dabei zeichnen sich entsprechende Reparaturinitiierungen in der gesprochenen Sprache durch ihre saliente prosodische Realisierung aus (z.B. durch hohes Tonhöhenregister, Erhöhung der Lautstärke oder eine starke Akzentuierung), was dem gesamten Initiierungsturn „Staunen“ bzw. „Überraschung“ als Nebenbedeutung verleiht (vgl. Kap. 2.3.2.4; Couper-Kuhlen/Selting 2018: 191-193). In der medialen Schriftlichkeit werden die oben erwähnten prosodischen Besonderheiten mithilfe unterschiedlicher graphischer Ressourcen emuliert, beispielsweise mithilfe der Iteration einzelner Zeichen wie in (161):

129 Bei fremdinitiierten Selbstreparaturen aus den untersuchten Kurznachrichtendialogen werden in den präsentierten Belegen jeweils die (vermutliche) Problemquelle sowie die Reparaturdurchführung fett markiert. 
(161) SMS-Dialog \#2374:

Schreiberin $A$

Schreiberin $B$

Wie viele leute seid ihr eigtl? Ist die vl voll oder nur so die hälfte?

Nachricht \#1 - 14.10.2013 - 14:24:05

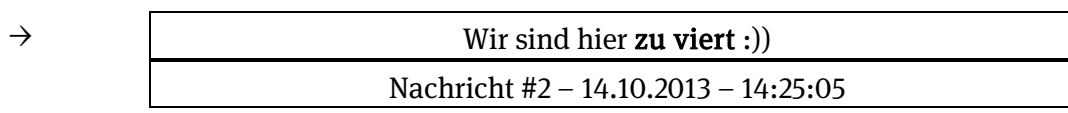

$\rightarrow$

Waass? Wieso zu viert?? Ich glaub du hast mich falsch verstanden :D

Nachricht \#3 - 14.10.2013-14:26:05

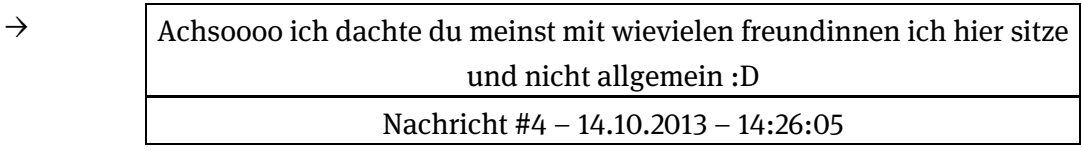

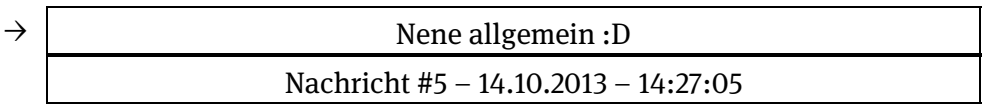

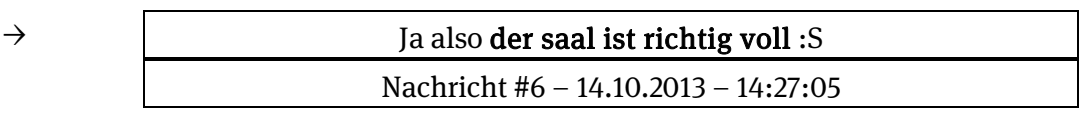

Dann halt mir bitte einen platz frei :)

Nachricht \#7 - 14.10.2013 - 14:28:05

Auf dem Weg zu einer Vorlesung fragt $A$ ihre Freundin, die bereits vor Ort ist, wie viele Studenten im Vorlesungssaal sitzen und ob dieser bereits voll ist. Die Nachricht wird jedoch von $B$ missverstanden, was vermutlich u.a. an der nicht-eindeutigen Personenreferenz „ihr“ (\#1) liegt. Da $B$ das Pronomen „ihr“ (\#1) nicht auf alle im Vorlesungssaal Anwesenden, sondern auf sich selbst und ihre Freundinnen bezieht, liefert sie in \#2 eine ihrem Verständnis entsprechende Anzahl der Personen, nämlich vier, was für eine Vorlesung eindeutig zu wenig wäre und bei $A$ ein Erwartungsproblem auslöst, welches von ihr in \#3 dargelegt wird. Die ersten zwei Nachrichten aus (161) weisen dabei die typische Struktur einer Reparatur in dritter Position (third position repair) im Sinne von Schegloff (1992) auf. Dabei sollte in \#3 eigentlich eine selbstinitiierte Selbstreparatur erfolgen, in der $A$ die richtige Lesart für ihre Frage in \#1 präsentiert. 
Stattdessen entscheidet sich $A$ für eine Fremdinitiierung, die sie in \#3 mit einer Kombination ${ }^{130}$ aus drei einzelnen Initiierungen konstruiert: Das durch die Iteration hervorgehobene „Waass?“(\#3) trägt dazu bei, das Erwartungsproblem zu signalisieren. Die Teilwiederholung mit dem Frageadverb wieso (,Wieso zu viert??“) richtet sich ebenso auf das Erwartungsproblem und trägt zur genauen Lokalisierung der Problemquelle bei. Letztendlich verweist der Deklarativsatz, der mit ich glaube eingeleitet ist, explizit auf ein aus $A$ 's Perspektive vorliegendes Missverständnis. In Nachricht \#4 signalisiert $B$ mit „Achsoooo“ die Veränderung in ihrem Verstehen und liefert eine Erklärung bzw. eine Rechtfertigung für ihre ursprüngliche Interpretation (,ich dachte du meinst mit wievielen freundinnen ich hier sitze") sowie kontrastiert diese mit der neuen, durch \#3 erzeugten Interpretation (,allgemein“) (vgl. Golato/Betz 2008; Imo 2009). Dieses alternative Verständnis wird in \#5 von $A$ bestätigt, woraufhin $B$ zur allerersten Nachricht zurückkehrt und ihre ursprüngliche Antwort revidiert, indem sie $A$ mitteilt, dass der Vorlesungssaal voll ist. Auffällig ist jedoch in diesem $\mathrm{Zu}$ sammenhang, dass eine andere Hervorhebungsstrategie, die sich v.a. für die Emulation der Lautstärke sehr gut eignet, nämlich die Majuskelschreibung, bei unspezifischen Fremdinitiierungen im Analysekorpus nicht vorkommt.

Bei der einzigen unspezifischen Fremdinitiierung im russischen Subkorpus handelt es sich ebenso um diejenige, die ein Erwartungsproblem thematisiert. In Nachricht \#1 aus (162) erkundigt sich Schreiber $A$ bei Schreiberin $B$, ob sie bereits eine Einkaufsliste für seinen bevorstehenden Junggesellenabschied zusammengestellt hat. Dabei geht $A$ davon aus, dass seine Bitte, eine Einkaufsliste für die Party zu erstellen, an $B$ weitergeleitet wurde, was allerdings nicht passiert zu sein scheint (vgl. \#8):

(162) Auszug aus dem SMS-Dialog \#3125:

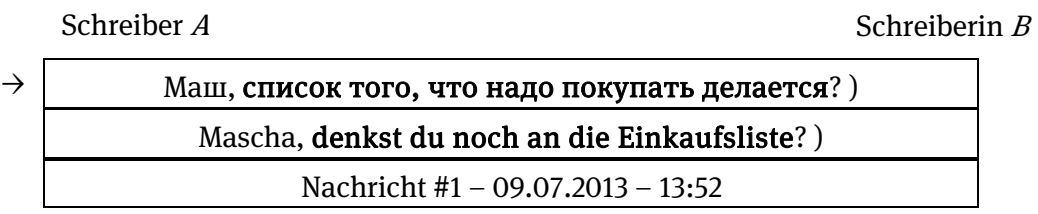

130 Dabei muss angemerkt werden, dass unspezifische Fremdinitiierungen im deutschen Subkorpus in fast 60\% der Fälle in einer Kombination mit einem spezifischeren Initiierungsformat, wie z.B. mit einer Teilwiederholung mit Fragewort, vorkommen. Das hat vermutlich damit zu tun, dass eine alleinstehende unspezifische Fremdinitiierung für den Rezipienten wenig aufschlussreich ist. Daher werden solche Reparaturinitiierungen oft zusammen mit ,stärkeren“ Formaten gekoppelt, die entweder in der gleichen Nachricht auftauchen oder in der Nachricht danach (zum Splitten von Nachrichten vgl. Beißwenger 2007: 245-253, 261-264; Imo 2015a). 


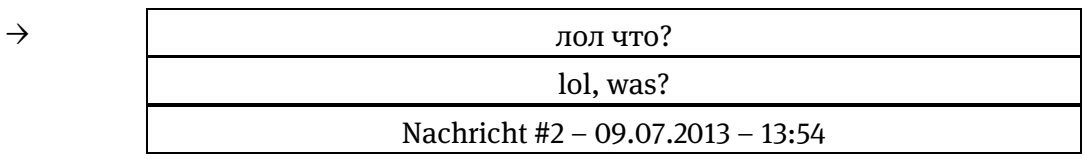

$\rightarrow$\begin{tabular}{c|} 
опа =) я просил тебя попросить составить список закупок на \\
сегодня)))
\end{tabular}

\begin{tabular}{|c|}
\hline ээээ. так. во первых, я это не могу \\
\hline äähm. Also, erstens kann ich das nicht \\
\hline Nachricht \#4 - 09.07.2013 - 13:56 \\
\hline
\end{tabular}

\begin{tabular}{|c|}
\hline Почему \\
\hline wieso \\
\hline Nachricht \#5 $-09.07 .2013-13: 56$ \\
\hline
\end{tabular}

\begin{tabular}{|c|}
\hline очень расстерянная и неорганизованная \\
\hline Ich bin sehr hektisch und unorganisiert \\
\hline Nachricht \#6 - 09.07.2013 - 13:57 \\
\hline
\end{tabular}

\begin{tabular}{|c|}
\hline почему \\
\hline wieso \\
\hline Nachricht \#7 $-09.07 .2013-13: 57$ \\
\hline
\end{tabular}

\begin{tabular}{|c|}
\hline во вторых первый раз слышу.хд \\
\hline Zweitens: Ich höre zum ersten Mal davon. xD \\
\hline Nachricht \#8 - 09.07.2013 - 13:57 \\
\hline
\end{tabular}

Da $B$ von der Bitte ihres Kommunikationspartners offensichtlich nichts mitbekommen hat, kann sie seine erste Nachricht in (162) in ihrem Wissensrahmen nicht richtig einordnen und initiiert daher mit dem unspezifischen Fragewort „что“ (,was') eine Reparatur. Neben dem Fragepronomen enthält die Initiierungsnachricht dabei auch das Akronym „лол“ (,lol'), das lediglich in diesem einen Dialog vertreten ist und analog zu Emojis sowie Emoticons (vgl. Pappert 2017: 196-198) in dem Fall eine Kommentarfunktion übernimmt. In der darauffolgenden Nachricht führt $A$ eine Selbstreparatur durch, indem er eine Erklä- 
rung für seine Nachfrage liefert, nämlich, dass er davon ausgegangen ist, dass $B$ von seiner Bitte gewusst hat, was, wie es sich nach einer Reihe weiterer - diesmal aber von $A$ - initiierter Reparaturen in Nachricht \#8 herausstellt, nicht der Fall war.

\section{Interjektionen}

Im Gegensatz zu unspezifischen Fragepronomen, die (zumindest im deutschen Korpus) auf das Vorhandensein eines Bedeutungsverstehens- oder eines Erwartungsproblems hinweisen, werden die Partikel hä sowie ihre Variante hö in den analysierten Daten ausschließlich bei Erwartungsproblemen eingesetzt. In (163) wird ein Auszug aus einem Dialog zwischen Schreiberin $A$, die sich in jenem Moment in Neuseeland aufhält, und Schreiberin $B$, die in Essen ist, präsentiert:

(163) Auszug aus dem WhatsApp-Dialog \#2624:

Schreiberin $A$

Schreiberin $B$

$\rightarrow$

Wann hast du nochmal nicht uni oder kannst so um 9 Skypen?

Nachricht \#8 - 08.12.2013 - 19:12:36

\begin{tabular}{|c|}
\hline Kannst jetzt gleixh? \\
\hline Nachricht \#9-08.12.2013-19:26:20 \\
\hline
\end{tabular}

\begin{tabular}{|c|}
\hline ?? \\
\hline Nachricht \#10 - 08.12.2013 - 19:29:02 \\
\hline
\end{tabular}

$\rightarrow$

\begin{tabular}{|c|}
\hline Muss in 15 Minuten zur Arbeit los fahren :D :D :D \\
\hline Nachricht \#11 - 08.12.2013 - 19:30:13 \\
\hline
\end{tabular}

$\rightarrow$

\begin{tabular}{|c|}
\hline Hä? \\
\hline Nachricht \#12-08.12.2013-19:30:28 \\
\hline
\end{tabular}

$\rightarrow$

\begin{tabular}{|c|}
\hline Ich hab schon Montag morgen :D \\
\hline Nachricht \#13 - 08.12.2013 - 19:30:39 \\
\hline
\end{tabular}

$\rightarrow$

Ach meinst morgen um 9

Nachricht \#14 - 08.12.2013-19:30:47

$\rightarrow$

\begin{tabular}{|c|}
\hline Ja genau für dich morgens und Mich abends \\
\hline Nachricht \#15-08.12.2013-19:31:23 \\
\hline
\end{tabular}




\begin{tabular}{|c|}
\hline Aso nee da kann ich nicht \\
\hline Nachricht \#16-08.12.2013 - 19:31:47 \\
\hline Uni... \\
\hline Nachricht \#17 - 08.12.2013 - 19:31:50 \\
\hline
\end{tabular}

Schreiberinnen $A$ und $B$ handeln in (163) den nächsten Termin für einen SkypeAnruf aus. In diesem Zusammenhang fragt $A$ zunächst danach, ob $B$ morgen früh zur Uni muss (nicht abgebildet). Nachdem $B$ dies bestätigt, erkundigt sich $A$ in \#8 danach, wann $B$ generell Zeit hat. Gleichzeitig unterbreitet sie aber einen konkreten Vorschlag für die Zeit eines potenziellen Skype-Termins, nämlich 9 Uhr. Dabei nimmt $B$ offensichtlich an, dass die angegebene Zeit sich auf die Ortszeit in A's Aufenthaltsort (also in Neuseeland) bezieht. Daher rechnet $B$ die Ortszeit für Deutschland aus, kommt auf $21 \mathrm{Uhr}$ und unterbreitet einen alternativen Vorschlag, kurz davor zu skypen, nämlich um ca. 19:30 nach deutscher und 7:30 nach neuseeländischer Zeit. Dieser Terminvorschlag wird von $A$ jedoch abgelehnt, was sie damit begründet, dass sie gleich zur Arbeit fahren muss. Somit baut sich für $B$ ein Widerspruch zwischen dieser Ablehnung und dem von $A$ ursprünglich vorgeschlagenen 9-Uhr-Skype-Termin auf, den sie in \#12 mithilfe der Partikel hä zum Ausdruck bringt. Die Reparaturdurchführung in \#13 deutet allerdings eher darauf hin, dass $A \# 11$ und nicht die problematische Zeitreferenz in \#8 als Problemquelle interpretiert hat, da sie die Erklärung dafür liefert, wieso sie gleich zur Arbeit fahren muss. Dennoch gibt diese Reparatur $B$ anscheinend einen Anstoß, ihre Interpretation der Zeitreferenz zu überdenken. Das passiert in Nachricht \#14, in der B nach dem Erkenntnisprozessmarker ach eine candidate reference „morgen um 9“ verschickt. Dabei präzisiert sie allerdings wieder nicht, ob sie 9 Uhr nach neuseeländischer oder deutscher Zeit meint, sondern verschiebt den Termin auf den nächsten Tag. In \#15 sichert $A$ das gegenseitige Verständnis, indem sie den nächsten Tag als gemeinten Termin bestätigt und außerdem klarstellt, dass die Zeitreferenz 9 Uhr als 9 Uhr morgens nach deutscher Zeit zu verstehen ist. Darauf reagiert $B$ zwar mit dem Erkenntnisprozessmarker „Aso“ (\#16), was die Wiederherstellung des gegenseitigen Verständnisses signalisiert, lehnt jedoch den von $A$ vorgeschlagenen Termin aus dem Grund ab, den sie bereits vor dem Auftreten der problematischen Zeitreferenz genannt hat (vgl. \#4-5 (nicht abgebildet) sowie „Uni...“ in \#17).

\section{Fragezeichen}

Neben Interjektionen sowie unspezifischen Fragewörtern, die in der gesprochenen Sprache sowie im Untersuchungskorpus als Fremdinitiierungen fungieren können, finden sich in den analysierten Daten auch solche Reparaturinitiierun- 
gen, die ausschließlich in der schriftbasierten Kommunikation vorkommen und funktional den unspezifischen Reparaturinitiierungen zugeordnet werden können, nämlich Fragezeichen (vgl. Kap. 3.5.2.1 sowie Höhn 2016; Mostovaia 2018, 2021). Dabei hat es sich gezeigt, dass Fragezeichen (zwölf Fälle im deutschen und vier im russischen Subkorpus; jeweils 4,4\% und 4,7\% aller Fremdinitiierungen) sowohl alleine als Fremdinitiierungen auftreten als auch mit anderen Initiierungsformaten kombiniert werden können.

Einen Beleg dafür, dass ein Fragezeichen in der Kurznachrichtenkommunikation zum Anzeigen des Nicht-Verstehens benutzt werden kann, liefert der Auszug (164), der einer Kommunikation zwischen einem Pärchen entnommen ist:

(164) Auszug aus dem SMS-Dialog \#3903:

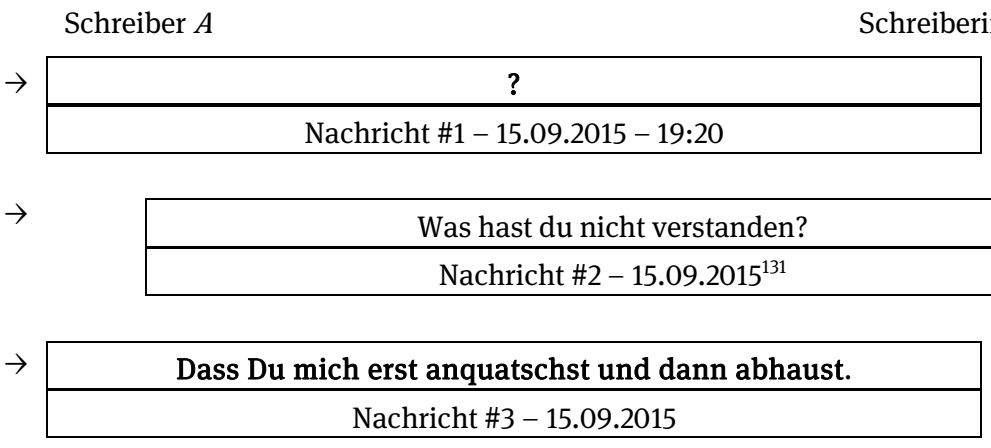

Der in die Datenbank MoCoDa eingetragene Dialog (164) beginnt direkt mit Nachricht \#1, sodass man über den Auslöser für das darin verschickte Fragezeichen lediglich anhand der Reparaturdurchführung in \#3 spekulieren kann. Hier steht jedoch ein anderer Aspekt im Fokus: Die in \#2 abgebildete Reparaturinitiierung expliziert B's Interpretation des in \#1 stehenden Fragezeichens als Ressource, mit der $A$ sein Nicht-Verstehen zum Ausdruck bringt. Diese Interpretation unterstützt also somit die in der vorliegenden Arbeit angenommene reparative Funktion von Fragezeichen.

In allen anderen Fällen der Reparatursequenzen, die Fragezeichen miteinbeziehen, sind die jeweiligen Problemquellen im Gegensatz zu (164) vorhanden und erkennbar. Bei den meisten davon handelt es sich um Bedeutungsverstehensprobleme, wie in (165):

131 In diesem Dialog ist lediglich die erste Nachricht mit den Zeitangaben versehen worden. 
(165) Auszug aus dem SMS-Dialog \#1958:

Schreiber $A$

Schreiberin $B$

\begin{tabular}{|c|c|}
\hline & Mittagessen gefunden :-) \\
\hline & Nachricht \#27 - 12.10.2012 - 22:00:05 \\
\hline$\rightarrow$ & $?$ \\
\hline & Nachricht \#28 - 12.10.2012 - 22:01:05 \\
\hline
\end{tabular}

$\rightarrow \quad$ Mc Donalds gefunden. VORNAME futtert 3 Burger mit einer grossen Pommes und Cola. so wie gestern. Doppel schlange quasi.

Nachricht \#29 - 12.10.2012 - 22:12:05

Aaachso. Ohne mampf kein kampf. Guten hunger zusammen. :)

Nachricht \#30 - 12.10.2012 - 22:14:05

Schreiber $A$ und Schreiberin $B$ sind ein Pärchen und machen getrennt Urlaub. $A$ ist gerade unterwegs und hat bereits im vorherigen Verlauf desselben Dialogs geschildert, dass er Hunger hat und nach einer McDonalds-Filiale Ausschau hält. In \#27 teilt $A$ seiner Freundin eine freudige Nachricht mit, endlich ein Mittagessen gefunden $z u$ haben, woraufhin $B$ mit einem Fragezeichen ihr Verstehensproblem signalisiert. Schreiber $A$ reagiert auf diese Fremdinitiierung in \#29, indem er das vermutlich problematische Nomen „Mittagessen“ (\#27) durch „Mc Donalds“ (\#29) ersetzt und somit eine thematische Verknüpfung zum vorherigen Verlauf des Dialogs herstellt (McDonalds wurde von ihm bereits in der hier nicht abgebildeten Nachricht \#22 erwähnt). Außerdem expandiert er seine Nachricht und liefert mehr Informationen zu seinem Aufenthalt bei McDonalds, sodass man diesen erweiterten Kontext ebenso als eine Art Hilfestellung zum Beheben des entstandenen Verstehensproblems deuten könnte.

Neben einzelnen Fragezeichen können auch iterierte Fragezeichen als Fremdinitiierungen fungieren. Außerdem werden Fragezeichen, wie bereits erwähnt, im Untersuchungskorpus auch mit anderen Initiierungsformaten kombiniert (vgl. (166) und (198)):

(166) Auszug aus dem WhatsApp-Dialog \#2614:

Schreiber $A$

Schreiberin $B$

$\rightarrow$

Ruf einfach an bevor du kommst, damit ich aus dem Krankenhaus zurück bin

Nachricht \#5 - 15.11.2013 - 12:20:00 


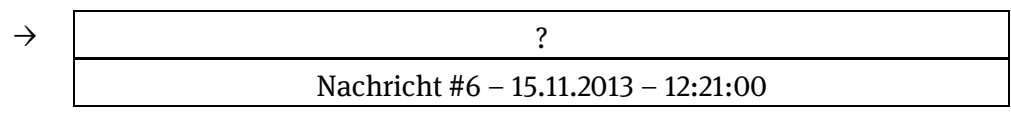

\begin{tabular}{|c|c|}
\hline \multirow[t]{2}{*}{$\rightarrow$} & Krankenhaus? Was ist los? \\
\hline & Nachricht \#7 - 15.11.2013 - 12:21:00 \\
\hline
\end{tabular}

\begin{tabular}{|c|c|}
\hline \multirow[t]{2}{*}{$\rightarrow$} & Besuche Lena \\
\hline & Nachricht \#8 - 15.11.2013 - 12:23:00 \\
\hline
\end{tabular}

\begin{tabular}{|c|}
\hline Ach du besuchst du die Lena. \\
\hline Nachricht \#9-15.11.2013 - 12:23:00 \\
\hline
\end{tabular}

\begin{tabular}{|c|}
\hline Stimmt. Gehts ihr gut? \\
\hline Nachricht \#10 - 15.11.2013 - 12:24:00 \\
\hline
\end{tabular}

In (166) bittet $A$ seine Kommunikationspartnerin, ihm bei der Anmeldung von Klausuren zu helfen. Schreiberin $B$ willigt ein und schlägt den kommenden Sonntag als Termin vor (nicht abgebildet). Nachdem $A$ dem Termin zugestimmt hat, weist $B$ ihn darauf hin, dass er vor seinem Besuch kurz anrufen soll, um sicher zu gehen, dass sie aus dem Krankenhaus zurück ist (vgl. \#5). Die Erwähnung des Krankenhauses löst eine Fremdinitiierung aus, die auf zwei Nachrichten \#6 und \#7 gesplittet wurde. Einer unspezifischen Fremdinitiierung in Form eines Fragezeichens folgt eine Teilwiederholung, die die Problemquelle exakt lokalisiert, sowie eine explizite Nachfrage nach dem Grund des Krankenhausbesuchs. In \#8 erklärt $B$, dass sie eine gemeinsame Bekannte von den beiden im Krankenhaus besuchen möchte.

Darüber hinaus werden Fragezeichen auch vereinzelt dafür benutzt, um die ausbleibende Reaktion einzufordern (vgl. \#10 aus (163)). Solche Fälle betrachten Schönfeldt/Golato (2003: 266) bei ihrer Analyse der Gruppenchatkommunikation im Kontext von Reparaturen, die sie als sequential repairs bezeichnen. In der vorliegenden Arbeit wird die Reaktionsanforderung allerdings nicht als eine Reparatur aufgefasst.

\subsubsection{Metakommunikativ explizite Fremdinitiierungen}

Die Gruppe der metakommunikativ expliziten Fremdinitiierungen ist im deutschen Subkorpus mit 29 (11\%) und im russischen mit zwölf Reparaturinitiierungen (14\%) vertreten. Diese werden von Rezipienten der Problemquelle in den untersuchten Daten eingesetzt, um Bedeutungsverstehens- und Referenzprobleme anzuzeigen. Das deutsche Teilkorpus enthält dabei meist satzwertige 
Fremdinitiierungen mit Verben wie verstehen (neun Tokens; vgl. (135), (197), (200), (209)), heißen (sieben Tokens; vgl. (168)), meinen (sechs Tokens; vgl. (217)), reden (zwei Tokens) und bedeuten (ein Token), während im russischen Subkorpus Fremdinitiierungen mit dem Verb понимать (,verstehen'; zwei Tokens; vgl. (167)) sowie der Präpositionalphrase в смысле (,im Sinne von/ich meine'; acht Tokens; vgl. (169), (170), (171)) vorliegen.

In den deutschen Kurznachrichten bilden Fremdinitiierungen mit dem negierten Verb verstehen die größte Gruppe im Rahmen des metakommunikativ expliziten Initiierungsformats (neun Fälle). Dabei wird verstehen meist in der 1.P. Sg. Präsens in Deklarativsätzen mit Verberststellung und Verbzweitstellung gebraucht (vgl. (135) und (197); für verstehen im Perfekt vgl. (200)). Im russischen Subkorpus finden sich zwei ähnliche Fälle mit dem Verb понимать (,verstehen'). Allerdings steht понимать (,verstehen') in den beiden Belegen im Gegensatz zu deutschen Beispielen nicht im Präsens, sondern im Präteritum:

(167) Auszug aus dem WhatsApp-Dialog \#3355:

Schreiber $A$

Schreiberin $B$

\begin{tabular}{|c|}
\hline В одноклассниках и в агенте [смайл] как сессия ? \\
\hline Und ich chatte in Odnoklassniki und Agent [Emoji]. Wie läuft es mit \\
deinen Prüfungen? \\
\hline Nachricht \#5 - 01.02.2012 - 20:39 \\
\hline
\end{tabular}

\begin{tabular}{|c|}
\hline У меня в декабре была, а у тя? \\
\hline Ich habe meine Prüfungen im Dezember abgelegt. Und bei dir? \\
\hline Nachricht \#6 - 01.02.2012 - 20:40 \\
\hline Тоже , два долга [смайл] сопромат и термех \\
\hline $\begin{array}{l}\text { h, habe zwei nicht bestanden [Emoji] Festigkeitslehre und } \\
\text { theoretische Mechanik }\end{array}$ \\
\hline Nachricht \#7 - 01.02.2012 - 20:41 \\
\hline ниче не поняла \\
\hline Ich habe nichts verstanden \\
\hline Nachricht \#8-01.02.2012 - 20:41 \\
\hline
\end{tabular}




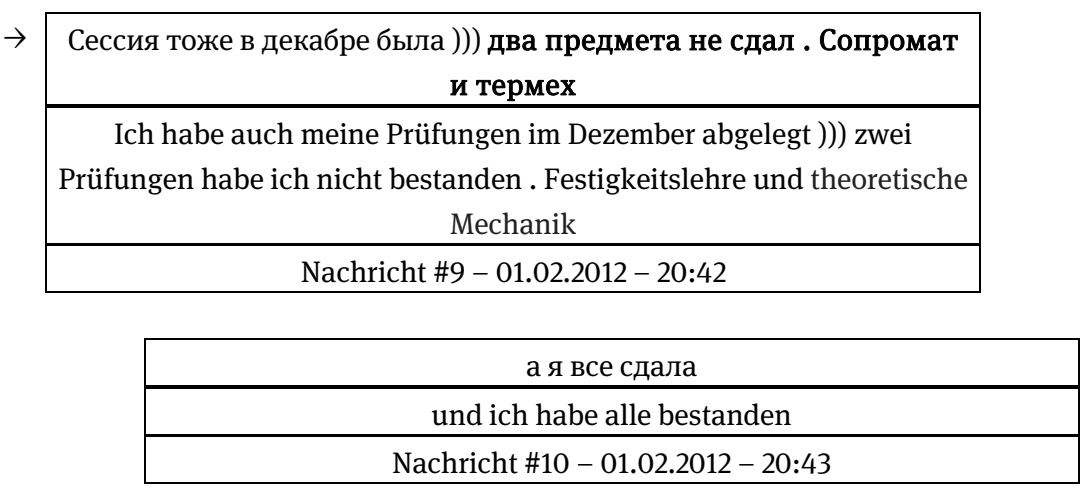

In (167) besprechen zwei Studierende (Schreiber $A$ und Schreiberin $B$ ) ihre Prüfungszeit. Als Antwort auf $A$ 's Frage teilt $B$ in \#6 mit, dass ihre Prüfungszeit bereits im Dezember gelaufen ist, liefert jedoch keine Informationen zu ihrem ,Erfolgsgrad' bei den Prüfungen und gibt die Frage zurück. $A$ berichtet, dass seine Prüfungszeit ebenfalls im Dezember war, fügt jedoch hinzu, dass er zwei Prüfungen nicht bestanden hat. Dabei handelt es sich um die Festigkeitslehre (сопротивление материалов $\rightarrow$ сопромат) und theoretische Mechanik (теоретическая механика $\rightarrow$ теормех $\rightarrow$ der einfacheren Aussprache zuliebe kann das $o$ weggelassen werden, sodass aus теормех термех entsteht). Nachricht \#7 löst allerdings bei $B$ ein Bedeutungsverstehensproblem aus, dessen Vorhandensein sie $A$ in \#8 signalisiert. Daraufhin baut $A$ die problematische Nachricht lexikalisch sowie syntaktisch aus. Dabei zeigt er, dass sich das Wort „тоже“ (,auch') auf die Zeit der Prüfungsperiode bezieht. Danach formuliert $A$ den eigentlich für Studierende geläufigen Ausdruck „два долга“ aus (wortwörtlich ,zwei Schulden', sinngemäß ,zwei Prüfungen, die man bei dem ersten Versuch nicht bestanden hat'), indem er schreibt, dass er zwei Prüfungen nicht bestanden hat und deren Bezeichnungen aus Nachricht \#7 wiederholt. Die darauffolgende Reaktion von $B$ zeigt, dass das Problem erfolgreich gelöst wurde.

Des Weiteren wurden in den deutschen Daten sechs Fremdinitiierungen mit der was heißt $x$-Konstruktion festgestellt, und zwar mit derer Variante, die Günthner (2015) als „klarifizierende fremd-responsive was heißt $x^{\text {- }}$ Konstruktion“ bezeichnet. Solche Reparaturinitiierungen fungieren „als Informationsrückfragen, mit denen Sprecher vom vorangegangenen Gesprächspartner weitere Erläuterungen bzw. Spezifikationen bzgl. der thematisierten Einheit , $x^{‘}$ einfordern“ (Günthner 2015: 198; vgl. auch Hrncal 2011). Dabei werden Fremdinitiierungen in vier von sechs Beispielen mit der Modalpartikel denn erweitert, was auch für die gesprochene Sprache belegt wurde (vgl. Hrncal 2011; Günthner 2015; zur Funktion von denn in der gesprochenen Sprache vgl. Dep- 
permann 2009). Die für Internetforen von Hrncal (2011) belegte Variante der Konstruktion was heißt $x$ in Kombination mit der Partikel schon kommt jedoch in den analysierten Fremdinitiierungen nicht vor.

Fremdinitiierungen mit der was heißt $x$-Konstruktion werden bei Bedeutungsverstehens- sowie Referenzproblemen eingesetzt. Ein Beispiel für ein damit angezeigtes Bedeutungsverstehensproblem findet sich im weiteren Verlauf der Arbeit in (210). An dieser Stelle wird der Gebrauch der was heißt $x^{-}$ Konstruktion im Falle einer problematischen Referenz veranschaulicht:

(168) Auszug aus dem SMS-Dialog \#1222:

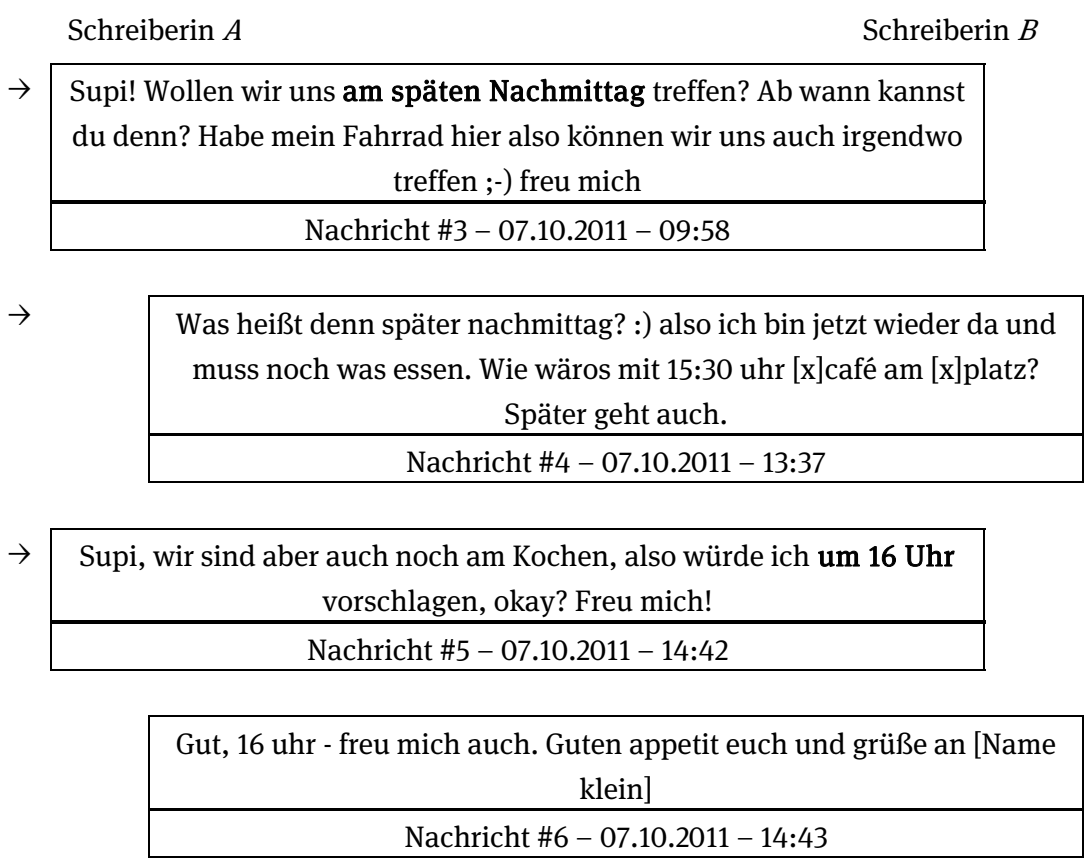

Schreiberin $A$ besucht ihren Freund, der in der gleichen Stadt wie eine ihrer Freundinnen, Schreiberin $B$, wohnt. Diese Gelegenheit nutzt $A$, um sich mit $B$ zu treffen. Daher unterbreitet sie ihr in \#3 ein Verabredungsangebot, in dessen Rahmen sie einen Termin vorschlägt, nämlich den späten Nachmittag. Da die von $A$ gelieferte Zeitangabe nicht eindeutig interpretierbar zu sein scheint, leitet $B$ in \#4 eine Reparatur ein, indem sie in Form einer was heißt denn $x$-Konstruktion mit der wiederholten problematischen Zeitreferenz deren Präzisierung einfordert. Gleichzeitig liefert $B$ selbst einen Terminvorschlag („15:30 uhr“), den man u.U. als eine Art candidate reference ansehen könnte. Die somit initiierte Reparatur wird von $A$ allerdings eher implizit nebenbei bearbeitet, da sie nach 
einer kurzen Begründung („wir sind aber auch noch am Kochen“) einen Gegenvorschlag macht, nämlich „16 Uhr“ (vgl. \#5). In \#6 wird der von $A$ vorgelegte Termin von $B$ bestätigt, sodass man das gegenseitige Verstehen als wiederhergestellt betrachten kann.

Analog zu was heißt $x$-Konstruktionen im Deutschen, die bei selbstinitiierten und fremdinitiierten Selbstreparaturen sowie bei fremdinitiierten Fremdreparaturen verwendet werden können (vgl. Günthner 2015), fungiert im Russischen die Konstruktion в смысле $+X$ ? (wortwörtlich: ,im Sinne (von)', sinngemäß: ,In welchem Sinne? Was meinst du mit $X$ ?/Was meinen Sie mit $X /$ Was meint ihr mit $X$ ? Was heißt $X$ ?'), die nicht nur bei Reparaturinitiierungen, sondern auch bei Reparaturdurchführungen eingesetzt wird (vgl. Goletiani 2003; für weitere Beispiele in der gesprochenen Sprache vgl. Bolden 2009; Afinogenova 2015; Gerasimova 2016). Im Kontext von fremdinitiierten Selbstreparaturen verweisen Fremdinitiierungen mit в смысле (,In welchem Sinne?‘) v.a. auf Referenz- sowie Bedeutungsverstehensprobleme (vgl. Goletiani 2003: 203-204). Die Analysedaten liefern fünf Beispiele, zwei von denen die Wiederholung der Problemquelle in der nachgestellten Position aufweisen. Neben Referenz- und Bedeutungsverstehensproblemen werden dabei auch Erwartungsprobleme bzw. u.U. auch problematische sequenzielle Implikationen thematisiert, wie in (169):

(169) Auszug aus dem WhatsApp-Dialog \#3623:

\begin{tabular}{|c|c|}
\hline \multicolumn{2}{|c|}{ Schreiberin $A$} \\
\hline \multirow[t]{3}{*}{$\rightarrow$} & Слушай ты красавчик какой) \\
\hline & Weißt du was, du bist ja cool) \\
\hline & 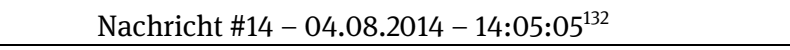 \\
\hline & В смысле? \\
\hline & In welchem Sinne? \\
\hline & Nachricht \#15 - 04.08.2014 - 14:05:05 \\
\hline \multirow[t]{3}{*}{$\rightarrow$} & Сколько баллов набрала [Emoji] \\
\hline & Du hast so viele Punkte gesammelt [Emoji] \\
\hline & Nachricht \#16 - 04.08.2014 - 14:05:05 \\
\hline
\end{tabular}

132 Vgl. Fußnote 117. 


\begin{tabular}{|c|}
\hline Мало .. \\
\hline Wenig .. \\
\hline Nachricht \#17 - 04.08.2014-14:05:05 \\
\hline
\end{tabular}

In (169) besprechen zwei Freundinnen Ergebnisse ihrer Aufnahmeprüfungen für einen Masterstudiengang, von denen abhängt, ob der Staat oder sie selbst ihr Studium finanzieren würden. Nachdem erwähnt wurde, dass $B$ genug Punkte bekommen hat, um sich ihr Studium von dem Staat finanzieren zu lassen, macht sie ihrer Freundin in \#14 ein Kompliment, das sich in dem Fall als Problemquelle herausstellt, was mindestens zwei Gründe haben könnte. Zum einen kann der (vermeintliche) Themenwechsel von $B$ 's Aufnahmeprüfung und ihren Studienplänen zu denen von $A$ eine problematische sequenzielle Implikation ausgelöst haben, sodass $A$ die Relevanz bzw. die Anknüpfung der Nachricht \#14 an den vorherigen WhatsApp-Verlauf nicht erkennen kann. Zum anderen kann das Entstehen eines Problems dadurch bedingt sein, dass $A$ inhaltlich zwar ,mitgeht', doch die Bewertungen von $A$ und $B$ hinsichtlich der von $A$ erreichten Punktezahl divergieren. Während $B$ versucht, ihre Freundin aufzumuntern und ihre Leistung als gut darzustellen, bleibt $A$ objektiv und schätzt die Anzahl ihrer Punkte eher als wenig ein, da ihr Name in der Liste mit Studienbewerbern nicht hoch genug steht, um nach dem aktuellen Stand einen Anspruch auf eine staatliche Finanzierung zu haben. In diesem Fall würde ein Erwartungsproblem vorliegen.

$\mathrm{Zu}$ den oben bereits erwähnten fünf Fällen von Reparaturinitiierungen in Form в смысле $(+X)$ ? (,In welchem Sinne? Was meinst du (mit $X)$ ?') kommen noch drei weitere Beispiele, bei denen dieselbe Konstruktion in der für die schriftbasierte computervermittelte Kommunikation typischen Kurzform BCM vorkommt (vgl. Zemzereva 2016; Vagenlyaitner/Luparevich 2017). Diese Form kommt durch eine Verschmelzung der Präposition $B($,in') mit dem auf die ersten zwei Buchstaben abgekürzten Nomen смысле (,Sinn-PREP`) zustande:

(170) Auszug aus dem WhatsApp-Dialog \#3361:

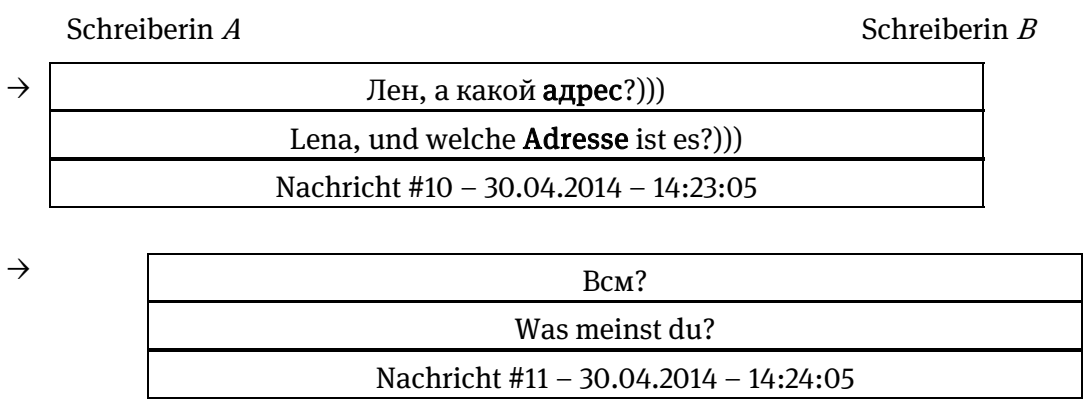




$\rightarrow \mid$\begin{tabular}{c|}
\hline ну где съёмка будет)) \\
\hline Wo das Foto-Shooting halt stattfindet)) \\
\hline Nachricht \#12 - 30.04.2014 - 14:25:05 \\
\hline
\end{tabular}

\begin{tabular}{|c|}
\hline [адрес] \\
\hline [Adresse] \\
\hline Nachricht \#13-30.04.2014-14:25:55 \\
\hline
\end{tabular}

Zu Beginn des Dialogs (170) diskutieren Schreiberinnen $A$ und $B$, welche Frisur zu einer Sportjacke passen würde, da sie sich, wie es sich in Nachricht \#12 herausstellen wird, auf ein Foto-Shooting vorbereiten. In \#10 wechselt Schreiberin $A$ jedoch das Thema und fragt unangekündigt nach einer Adresse, woraufhin $B$ mit der Fremdinitiierung „Всм?“ (,Was meinst du?`) reagiert. Diese Reparaturinitiierung wird von $A$ mit der in den Kontext nicht eindeutigen Referenz „адрес“ (,Adresse'), die sich potenziell auch auf die Adresse des Friseursalons beziehen könnte, in Verbindung gebracht und in \#12 entsprechend bearbeitet. Dabei liefert $A$ zur Problemquelle ein Attribut in Form eines Relativsatzes, das klarstellt, dass sie die Adresse gemeint hat, wo das Foto-Shooting stattfinden wird. Dadurch ist die Intersubjektivität wiederhergestellt, da $B$ ihr Verstehen anschließend durch das Verschicken der eingeforderten Information, nämlich der Adresse, signalisiert.

Im Gegensatz zur Vollform в смысле $(+X)$ ? (,In welchem Sinne? Was meinst du (mit $X)$ ?`) wird $в с м$ ? nicht mit der wiederholten Problemquelle kombiniert. Die Frage, ob solche Kombinationen möglich sind oder nicht, muss in zukünftigen Untersuchungen geklärt werden. Im Analysekorpus findet sich lediglich ein Fall (171), wo die Problemquelle durch eine dem $B C M$ folgende Erklärung konkret angesprochen wird. Dabei stellt sich jedoch die Frage, ob diese Erklärung mit $B C M$ eine Einheit bildet oder ob es sich dabei um eine zweite Fremdinitiierung handelt:

(171) Auszug aus dem WhatsApp-Dialog \#3346:

Schreiberin $A$

Schreiberin $B$

\begin{tabular}{|c|}
\hline ты тоже к ней не пойдешь?)) \\
\hline Gehst du dann zu ihrem Geburtstag auch nicht?)) \\
\hline Nachricht \#16 - 29.04.2014 - 14:28:05 \\
\hline
\end{tabular}

\begin{tabular}{|c|}
\hline X3 \\
\hline Weiß der Geier \\
\hline Nachricht \#17 $-29.04 .2014-14: 29: 05$ \\
\hline
\end{tabular}




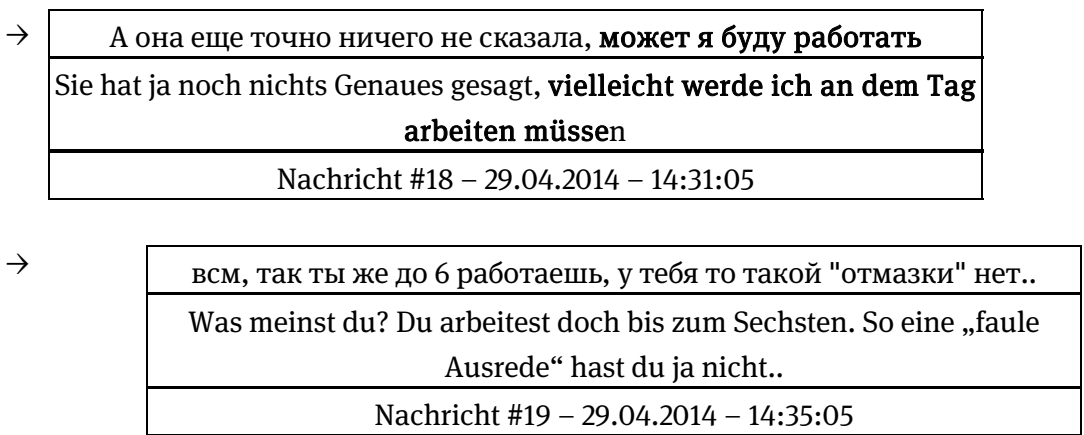

$\rightarrow \mid$\begin{tabular}{|c|}
\hline Я не знаю до какого работаю \\
\hline Ich weiß nicht, bis wann ich arbeite \\
\hline Nachricht \#20 - 29.04.2014 - 14:37:05 \\
\hline
\end{tabular}

Schreiberinnen $A$ und $B$ lästern über eine gemeinsame Freundin, die eine Einladung zu $A$ 's Geburtstagsfeier abgelehnt hat und ihre Ablehnung damit begründet hat, dass sie an dem Tag arbeiten muss. Sowohl $A$ als auch $B$ halten diesen Grund für eine faule Ausrede, daher zeigt sich $A$ etwas beleidigt. In \#16 fragt $B$, ob $A$ im Gegenzug zur Geburtstagsparty dieser Freundin auch nicht hingeht, woraufhin $A$ noch keine eindeutige Antwort geben kann, da sie noch nicht weiß, ob sie an dem Tag arbeiten muss. Diese Begründung löst bei $B$ ein Erwartungsproblem aus, da sie glaubt, dass $A$ bis zum Sechsten arbeiten muss und daher ihre Arbeit nicht als Vorwand für die Ablehnung dieser Einladung verwenden kann. Da $A$ über den $[\mathrm{K}+]$-Status über ihren Job (vgl. Heritage/Raymond 2005) verfügt, relativiert sie $B$ 's Behauptung und bestätigt gleichzeitig ihre eigene Aussage in \#18, indem sie eine Reparatur in \#20 durchführt und offenlegt, dass sie selbst noch nicht weiß, bis wann sie arbeiten wird.

\subsubsection{Fragewörter und Fragewörter mit Teilwiederholungen}

Bei analysierten Fremdinitiierungen in Form eines Fragewortes bzw. eines Fragewortes mit Teilwiederholung lässt sich eine Reihe von Gemeinsamkeiten mit entsprechenden Fremdinitiierungen in der gesprochenen Sprache erkennen. Das betrifft sowohl die Wahl der Interrogativa als auch den Einsatz dieser Formate bei bestimmten Problemquellen. Die Übersicht der in den beiden Teilkorpora gefundenen Fragewörter, die sowohl alleine als auch mit Teilwiederholung eine Reparatur einleiten können, ist in Tabelle 8 dargestellt. 
Tab. 8: Fragepronomen und -adverbien in Formaten Fragewort (FW) sowie Fragewort mit Teilwiederholung $(F W T W)$ im russischen und deutschen Subkorpus

\begin{tabular}{llll}
\multicolumn{3}{c}{ Deutsche Fremdinitiierungen } \\
\hline & FW & & FWTW \\
\hline was & 9 & welch- & 7 \\
wo & 5 & wieso & 6 \\
wieso & 4 & was für & 5 \\
wann & 3 & was & 4 \\
warum & 3 & warum & 3 \\
welch- & 3 & wie & 2 \\
wie & 3 & wofür & 2 \\
wem & 1 & wo & 1 \\
wofür & 1 & wohin & 1 \\
wohin & 1 & inwiefern & 1 \\
& 33 & & 32 \\
& $(12,2 \%)$ & & $(11,8 \%)$ \\
\hline
\end{tabular}

\begin{tabular}{|c|c|c|c|}
\hline \multicolumn{4}{|c|}{ Russische Fremdinitiierungen } \\
\hline & FW & & FWTW \\
\hline $\begin{array}{l}\text { почему } \\
(\text {,warum') }\end{array}$ & 6 & $\begin{array}{l}\text { как- } \\
\text { (,welch-') }\end{array}$ & 8 \\
\hline $\begin{array}{l}\text { как- } \\
\text { (,welch-`) }\end{array}$ & 3 & $\begin{array}{l}\text { почему } \\
\text { (,warum') }\end{array}$ & 2 \\
\hline что (,was') & 3 & $\begin{array}{l}\text { что } \\
\text { (,was') }\end{array}$ & 1 \\
\hline $\begin{array}{l}\text { куда } \\
\text { (,wohin') }\end{array}$ & 2 & $\begin{array}{l}\text { куда } \\
\text { (,wohin') }\end{array}$ & 1 \\
\hline $\begin{array}{l}\text { сколько } \\
\text { (,wie viel') }\end{array}$ & 1 & где (,wo') & 1 \\
\hline \multirow[t]{3}{*}{$\begin{array}{l}\text { зачем } \\
(, \text { wieso' })^{134}\end{array}$} & 1 & $\begin{array}{l}\text { зачем } \\
\text { (,wieso') }\end{array}$ & 1 \\
\hline & & $\begin{array}{l}\text { кто } \\
\text { (,wer') }\end{array}$ & 1 \\
\hline & $\begin{array}{l}16 \\
(18,8 \%)\end{array}$ & & $\begin{array}{l}15 \\
(17,6 \%)^{135}\end{array}$ \\
\hline
\end{tabular}

Aus der tabellarischen Darstellung (Tab. 8) der im Untersuchungskorpus verwendeten Fragewörter geht hervor, dass in den beiden Sprachen eine ganze Reihe von Fragepronomen sowie -adverbien als (Teile von) Fremdinitiierungen eingesetzt werden. Bei dem Vergleich der Verteilung von Interrogativa auf die beiden Formate in der jeweiligen Sprache weist nur das $w$-Determinativ was für eine systematisch bedingte, nur auf das Format Fragewort mit Teilwiederholung beschränkte Distribution auf. Während alle anderen Fragewörter in der jeweiligen Sprache zumindest potenziell für beide Formate denkbar sind, erfordert das w-Determinativ was für (Zifonun/Hoffmann/Strecker 1997: 36) eine Nominalphrase, die in den vorliegenden Daten dem Reparandum entspricht, und fungiert somit immer nur im Kontext des Formats Fragewort mit Teilwiederholung.

133 Почему entspricht außerdem den deutschen wieso, weshalb und weswegen.

134 Зачем kann ins Deutsche auch als ,wieso', ,wozu', ,wofür‘ etc. übersetzt werden.

135 Die Prozentzahlen in Tabelle 8 zeigen den Anteil der Fremdinitiierungen im Format Fragewort und Fragewort mit Teilwiederholung in Bezug auf die Gesamtzahl der Fremdinitiierungen im jeweiligen Subkorpus (vgl. Fußnote 125). 
Die von Podlesskaya (2007) für das Russische beschriebene parallele Konstruktion что за $+X$ (,was für $+\mathrm{X}^{`}$ ) wurde in den russischen Daten nicht gesichtet. In zwei Fällen wurde die Fremdinitiierung mit was für $X$ in den deutschen Kurznachrichten durch die Partikel denn erweitert, die direkt nach was eingefügt wurde (vgl. (172)):

(172) Auszug aus dem Dialog \#2344:

Schreiber $A$

Schreiberin $B$

\begin{tabular}{|c|}
\hline Hi :P \\
\hline Nachricht \#1 $-28.10 .2013-20: 07$ \\
\hline
\end{tabular}

\begin{tabular}{|c|}
\hline :D :D hi \\
\hline Nachricht \#2 - 28.10.2013- 20:07 \\
\hline
\end{tabular}

\begin{tabular}{|c|}
\hline Wie geht's dir? \\
\hline Nachricht \#3-28.10.2013-20:07 \\
\hline
\end{tabular}

\begin{tabular}{c|c|}
\hline \multicolumn{1}{c|}{ Ganz gut :) und dir? } \\
\hline Nachricht \#4 - 28.10.2013 - 20:09 \\
\hline Den Umständen entsprechend ${ }^{\wedge \wedge}$ Also gerade gut :D \\
\hline Nachricht \#5 - 28.10.2013 - 20:10 \\
\hline Was denn für Umstände? \\
\hline Nachricht \#6 - 28.10.2013 - 20:10
\end{tabular}

$\rightarrow$

Abschlussprüfung in einem Monat.Vorbereitungskurs.Schule.

Klausuren.Präsentationen. Und viel Arbeit ${ }^{\wedge} \wedge$

Nachricht \#7 - 28.10.2013-20:12

Oh gott dann drück ich dir mal ganz fest die daumen :)

Nachricht \#8 - 28.10.2013- 20:12

In der Eröffnungsphase des Dialogs (172) tauschen befreundete Schreiber $A$ und Schreiberin $B$ Begrüßungsfloskeln sowie Wie geht's-Fragen aus. Während $B$ mit der allgemeinen Phrase „Ganz gut“ ihre aktuelle Lebenssituation charakterisiert, liefert $A$ seinerseits eine längere Beschreibung, in der er seine Verwendung von gut relativiert und in Beziehung zu irgendwelchen Umständen setzt, 
die er jedoch nicht weiter ausführt. Die von $A$ erwähnten Umstände greift $B$ in ihrer Fremdinitiierung auf und definiert sie somit als Problemquelle (vgl. \#6). Das von $B$ eingesetzte Initiierungsformat was für $X$ soll in dem Fall anzeigen, dass $B$ nähere Informationen zu dem für sie problematischen Ausdruck braucht. Diese liefert $A$ in \#7, indem er seine Umstände in Form einer Liste aufzählt.

Aus einer sprachvergleichenden Perspektive weist das deutsche Subkorpus eine größere Variation hinsichtlich der verwendeten Fragewörter auf, hier kommt beispielsweise das Interrogativadverb wie vor, das in den analysierten russischen Fremdinitiierungen nicht vertreten ist. Allerdings muss beachtet werden, dass in absoluten Zahlen das deutsche Subkorpus zweimal so viele Fremdinitiierungen mit Fragewörtern (inkl. Fragewörter mit Teilwiederholungen) als das russische aufweist, sodass die Variation der Fragewörter in der russischen schriftbasierten informellen Kommunikation weiter anhand einer größeren Datenkollektion untersucht werden muss. Funktional werden die Formate Fragewort sowie Fragewort mit Teilwiederholung sowohl im Russischen als auch im Deutschen ähnlich wie in der gesprochenen Sprache v.a. zum Signalisieren von Referenz- sowie Erwartungsproblemen eingesetzt.

In den beiden analysierten Subkorpora treten jeweils die Interrogativpronomen welch- und как-(,welch-') auf, die im Gegensatz zu ihrem englischen Analog which (vgl. Kendrick 2015: 171) auch alleine gebraucht werden können und außerdem mit der Problemquelle in Kasus, Genus und Numerus kongruieren, wodurch das Reparandum präziser lokalisiert wird. Dabei beziehen sich Fremdinitiierungen mit den alleinstehenden Pronomen welch- und как(,welch-') auf Referenzprobleme, während die Kombination mit dem Reparandum sowohl Referenz- als auch Erwartungsprobleme markieren kann (vgl. für die gesprochene Sprache vgl. Golato 2013):

(173) Auszug aus dem SMS-Dialog \#3052:

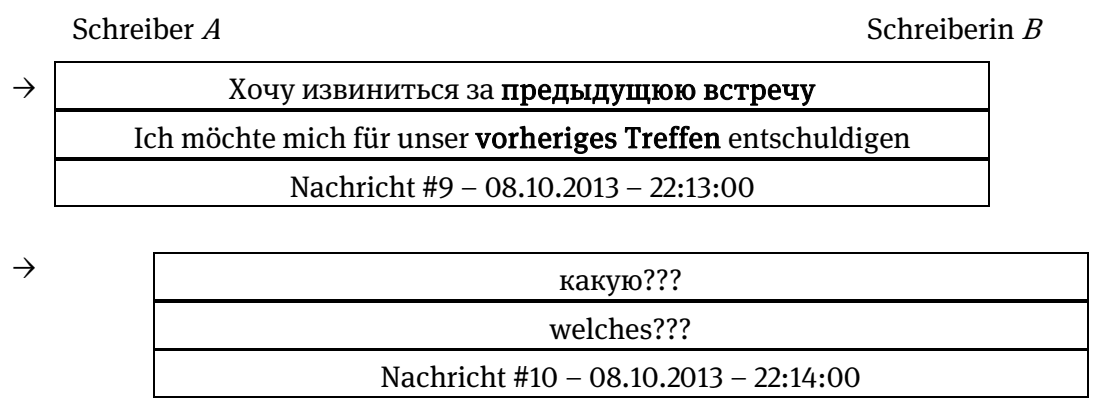


Das im Sommer. Es ist schon gut, dass du dich nicht daran erinnerst...

Nachricht \#11 - 08.10.2013 - 22:15:00

\begin{tabular}{c}
$\begin{array}{c}\text { нет, ну ты скажи..) я все помню)) но что же тебе показалось таким, } \\
\text { что потом нужно за это извеняться.? }\end{array}$ \\
\hline $\begin{array}{c}\text { Nein, sag mal..) ich erinnere mich an alles)) aber was ist dir denn } \\
\text { damals so schlimm vorgekommen, dass du dich jetzt dafür } \\
\text { entschuldigen musst.? }\end{array}$ \\
\hline Nachricht \#12 - 08.10.2013 - 22:22:00
\end{tabular}

Dialog (173) beginnt damit, dass Schreiber $A$ in \#1 ein Verabredungsangebot unterbreitet, das von Schreiberin $B$ jedoch abgelehnt wird, was sie mit Zeitmangel begründet (vgl. dafür (110)). In \#9 eröffnet $A$ ein neues Thema, indem er sich bei Schreiberin $B$ für ihr vorheriges Treffen entschuldigt. Für Schreiberin $B$ reicht der deiktische Verweis „предыдущюю“ (,vorherige') offensichtlich nicht aus, um das von $A$ gemeinte Treffen zu identifizieren. Daher initiiert sie mithilfe des Interrogativpronomens „какую“ (,welche`), kombiniert mit iterierten Fragezeichen, eine Reparatur. Dabei stimmt das Pronomen „какую“ (,welche-AKK.SG.FEM`) mit dem Nomen „встречу“ (,Treffen-AKK.SG.FEM`) in Kasus, Genus und Numerus überein, wodurch die Wiederholung des Nomens nicht notwendig ist, da die Problemquelle durch die morphologische Markierung präzise genug angezeigt wird. Als Reaktion darauf liefert $A$ lediglich einen Zeitraum für das angesprochene Treffen und lässt eine weitere potenziell notwendige Erklärung demonstrativ , unter den Tisch fallen' unter dem Vorwand, dass es umso besser ist, dass $B$ dieses Treffen vergessen hat.

Die Erwähnung eines Treffens löst auch in (174) ein Verständigungsproblem aus. Dabei geht es allerdings nicht um ein bereits vergangenes, sondern um ein bevorstehendes Treffen, das Schreiberin $B$ vergessen hat:

(174) Auszug aus dem SMS-Dialog \#3308:

Schreiber $A$

Schreiberin $B$

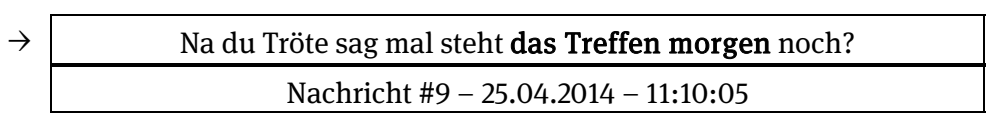

$\rightarrow$

Aloha :) hä? welches treffen? Wollten wir was machen?

Nachricht \#10 - 25.04.2014 - 11:11:11 
JA NATÜRLICH VERDAMMT :D ich hab mir schon gedacht, dass du es verplant hast :D Wollten doch Django gucken man!

Nachricht \#11 - 25.04.2014 - 11:13:15

Aaaaach mensch, du hast recht! ja also ich habe es zwar um ehrlich zu sein vergessen aber zeit habe ich trotzdem! :D

Nachricht \#12 - 25.04.2014 - 11:15:17

Im Gegensatz zu (173) weist die Fremdinitiierung in (174) jedoch auf ein Erwartungsproblem hin. Dieser Effekt wird zusätzlich durch die Verwendung der Interjektion „hä“ (\#10) als unspezifischer Fremdinitiierung sowie durch einen expliziten Hinweis darauf, dass $B$ nicht von einem geplanten Treffen ausgeht, verstärkt.

Darüber hinaus kann eine Teilwiederholung als syntaktischer Anker (vgl. Auer/Pfänder 2007; Pfeiffer 2015) oder frame fungieren (vgl. Schegloff 2013; Kendrick 2015). In (175) fragt Schreiber $A$ einen Freund, ob er zur Eröffnung einer Halle hingeht, und unterbreitet somit ein subtiles Verabredungsangebot:

(175) Auszug aus dem SMS-Dialog \#4011:

Schreiber $A$

Schreiber $B$

$\rightarrow \quad$ Hey kommst du heute zur eröffnung von der Halle? Ist auch umsonst;)

Nachricht \#1 - 27.02.2016 - 15:42:00

$\rightarrow$

Von was?

Nachricht \#2 - 27.02.2016 - 15:44:01

$\rightarrow$ Erstes linkes Zentrum in Bielefeld. Direkt an der uni. Livemusik und viele menschen;)

Nachricht \#3-27.02.2016 - 15:45:08

\begin{tabular}{|c|}
\hline Um wie viel Uhr wollt ihr dahin? \\
\hline Nachricht \#4 - 27.02.2016 - 15:45:22 \\
\hline
\end{tabular}

So zwischen 6 und 7

Nachricht \#5 - 27.02.2016 - 15:46:29

Dem Verabredungsvorschlag in \#1 folgt jedoch nicht der erwartbare zweite Teil der Paarsequenz in Form einer Zusage oder Ablehnung, sondern eine Fremdinitiierung. Die Lokalisierung des Reparandums wird dabei durch die Wiederho- 
lung der Präposition von aus der ursprünglichen Nachricht mit der Problemquelle gewährleistet. Somit wird der syntaktische Anker geschaffen, der darauf hinweist, dass sich das Fragepronomen „was“ auf die problematische Referenz, nämlich „Halle“, und nicht auf ein anderes Nomen aus \#1 bzw. auf die gesamte Nachricht bezieht (vgl. das Beispiel The what? bei Kendrick 2015: 170).

Im Vergleich zu diesen Fällen lässt sich bei Beispielen mit den Frageadverbien wie warum, wieso oder wie für das Deutsche sowie почему (,wieso/warum') für das Russische nicht immer eindeutig bestimmen, ob jeweils eine Reparaturinitiierung oder eine Nachfrage vorliegt. Für die Analyse wurden lediglich diejenigen Fälle ausgewählt, bei denen im gegebenen Kontext ein Widerspruch zum Wissensrahmen bzw. den Erwartungen des Rezipienten ersichtlich ist und/oder bei denen die Reaktion auf die jeweilige Frage als eine Reparaturdurchführung interpretiert werden kann (vgl. Selting 1987a; Egbert/Vöge 2008; Egbert 2009; Weber 2014).

Im folgenden Auszug (176) aus einem Dialog zwischen zwei Freunden erkundigt sich Schreiber $B$, ob Schreiber $A$ noch eine Karte für ein BundesligaFußballspiel braucht:

(176) Auszug aus dem SMS-Dialog \#2104:

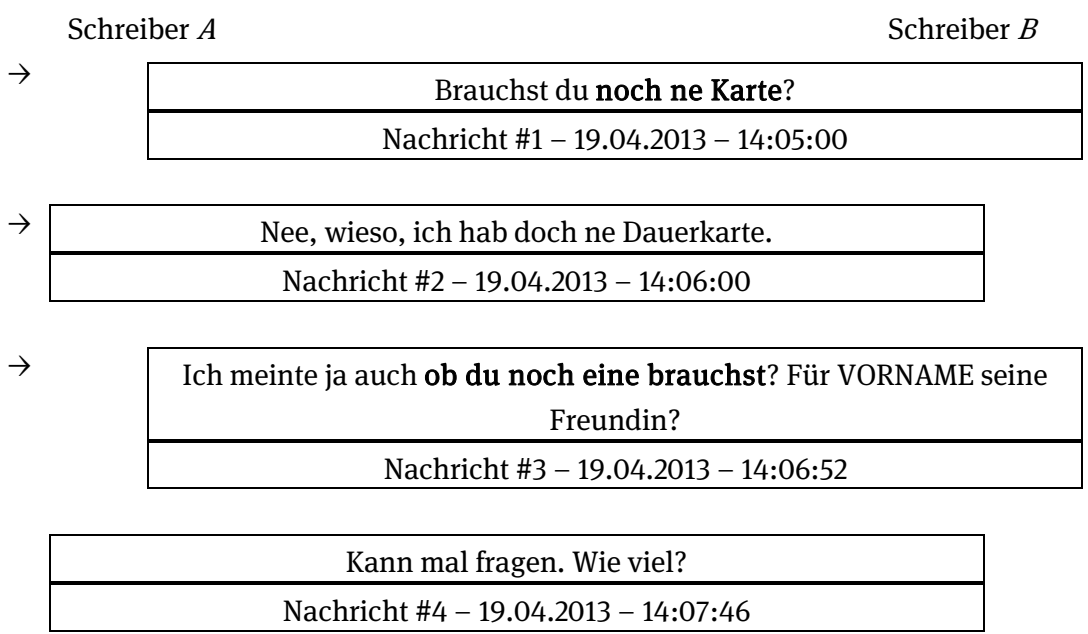

Die in \#1 abgebildete Nachricht lässt sich aufgrund der Phrasenstruktur auf zwei Arten interpretieren (vgl. Imo 2016 zur Ambiguität und Phrasenstruktur). Zum einen können „noch“ und „ne Karte“ zwei voneinander unabhängige Phrasen bilden, sodass „noch“ im Sinne von ,momentan, zurzeit“ gebraucht wird. Zum anderen könnte „noch“ der Nominalphrase mit dem Kopf „Karte“ untergeordnet sein, in dem Fall wird eine zusätzliche Karte gemeint. Nachricht \#2 legt 
offen, dass Schreiber $A$ sich für die erste Lesart entschieden hat. Da $A$ aber eine Dauerkarte besitzt und anscheinend davon ausgeht, dass Schreiber $B$ das auch weiß, löst $B$ ’ $s$ Nachfrage bei ihm ein Erwartungsproblem aus, welches er in \#2 thematisiert. Dabei setzt $A$ nach der Negationspartikel „Nee“, die als Ablehnung des (potenziellen) Angebots von $B$ gedeutet werden kann, das Interrogativadverb „wieso“ als Fremdinitiierung ein und liefert anschließend eine Erklärung, die die Ablehnung rechtfertigt und gleichzeitig implizit seine Interpretation von \#1 wiedergibt. In \#3 wird Nachricht \#1 von $B$ disambiguiert, indem er mit „noch eine“ die von ihm ursprünglich intendierte Bedeutung (nämlich ,eine zusätzliche Karte') klarstellt. Zudem liefert $B$ im Anschluss daran noch einen expliziten Hinweis auf den potenziellen Interessenten für die Karte aus $A$ 's Freundeskreis.

Während in (176) ein vorliegendes Erwartungsproblem mithilfe einer Fremdinitiierung in Form eines Fragewortes angezeigt wird, wird der Gebrauch des Initiierungsformats Fragewort mit Teilwiederholung anhand von (177) aus dem russischen Teilkorpus veranschaulicht:

(177) Auszug aus dem WhatsApp-Dialog \#3618:

Schreiberin $A$

Schreiberin $B$

\begin{tabular}{|c|}
\hline 즐 Игорь не офигел от количества ягоды?) \\
\hline War Igor von der Menge an Beeren nicht verblüfft?) \\
\hline Nachricht \#49-23.07.2014 - 14:05:05 ${ }^{136}$ \\
\hline
\end{tabular}

$\rightarrow$

\begin{tabular}{|c|}
\hline $\begin{array}{c}\text { Офигел))) сказал, что это странный подарок и не знает, как он ее } \\
\text { съест }\end{array}$ \\
\hline $\begin{array}{c}\text { War er))) Er hat gesagt, dass das ein komisches Geschenk ist und er } \\
\text { nicht weiß, wie er alle Beeren aufessen soll }\end{array}$ \\
\hline Nachricht \#50 - 23.07.2014-14:05:05 \\
\hline
\end{tabular}

$\rightarrow$

\begin{tabular}{|c|}
\hline Почему странный? \\
\hline Wieso komisch? \\
\hline Nachricht \#51 - 23.07.2014 - 14:05:05 \\
\hline
\end{tabular}

$\rightarrow$

Он не ожидал))

Er hat sowas einfach nicht erwartet))

Nachricht \#52 - 23.07.2014 - 14:05:05

136 Vgl. Fußnote 117. 
Im vorherigen Verlauf des Dialogs haben Freundinnen $A$ und $B$ einen Termin vereinbart, da $B$ bei $A$ Beeren für ihren Freund kaufen wollte (vgl. zwei weitere Auszüge aus demselben Dialog in (149) und (155)). Nachdem das Treffen, bei dem der Kauf abgeschlossen wurde, vorbei war, erkundigt sich $A$ bei ihrer Freundin, wie $B$ 's Freund auf die große Menge von Beeren reagiert hat. Darauf antwortet Schreiberin $B$, dass ihr Freund Beeren als Geschenk seltsam fand. In \#51 greift Schreiberin $A$ den aus ihrer Sicht offensichtlich problematischen Ausdruck „странный“ (,komisch/seltsam') auf und produziert eine Fremdinitiierung in Form eines Fragewortes mit Teilwiederholung. Damit macht sie deutlich, dass diese Bewertung des ,Beeren-Geschenks“ ihren Vorstellungen über das Konzept ,komische Geschenke' widerspricht. Nachdem $A$ ihr Problem thematisiert hat, liefert $B$ in \#52 eine Erklärung für die Wortwahl bei der Beschreibung der Reaktion ihres Freundes auf das Geschenk.

\subsubsection{Teilwiederholungen, vollständige Wiederholungen und nicht abgeschlossene Wiederholungen}

Teilwiederholungen kommen im Untersuchungskorpus nicht nur als Bestandteil anderer Formate wie z.B. Fragewort mit Teilwiederholung, sondern auch als eigenständiges Initiierungsformat vor. Dabei finden sich im deutschen Subkorpus 22 solcher Fremdinitiierungen (8\%), während die russischen Daten lediglich vier Fälle (5\%) aufweisen, von denen drei allerdings aus demselben Dialog stammen. Im russischen Korpus - evtl. wegen der insgesamt geringen Anzahl der Belege für das Format - kommen alle Teilwiederholungen alleine vor, in den deutschen Daten dagegen finden sich sowohl alleinstehende Teilwiederholungen als auch solche in Kombination mit anderen Formaten (vgl. (166) und (180)). In beiden Teilkorpora werden Teilwiederholungen v.a. bei Bedeutungsverstehensproblemen und gelegentlich bei Erwartungsproblemen eingesetzt.

Ein Auszug aus dem russischen Dialog, der wie erwähnt, drei Teilwiederholungen beinhaltet und in dem Schreiberin $B$ über das von ihr besuchte koreanische Restaurant berichtet, findet sich in (178). Der abgebildete Ausschnitt präsentiert lediglich zwei Teilwiederholungen (\#49 und \#52), die dritte Teilwiederholung aus dem ersten Teil des Dialogs wird in Kapitel 6.2.4 (vgl. (225)) diskutiert: 
(178) Auszug aus dem WhatsApp-Dialog \#4354:

Schreiberin $A$

Schreiberin $B$

$\rightarrow \quad$\begin{tabular}{c}
$\begin{array}{c}\text { Пибимбап-это короче микс различных овощей(в том числе } \\
\text { маринованных по особенному), говядины, яичницы и спец. } \\
\text { Острого соуса. Тебе ни продают и ты все это перемешиваешь... } \\
\text { Сложность приготовления в этих овощях }\end{array}$ \\
\hline $\begin{array}{c}\text { Bibimbap ist so eine Mischung aus unterschiedlichen Gemüsesorten } \\
\text { (auch auf eine besondere Weise eingelegt), Rindfleisch, einem Spiegelei } \\
\text { und einer speziellen scharfen Soße. Die werden dir nicht verkauft und } \\
\text { du mischst das... Am schwierigsten ist es, dieses Gemüse zuzubereiten }\end{array}$ \\
\hline Nachricht \#47 - 15.02.2018 - 15:56 \\
\hline
\end{tabular}

$\rightarrow$

\begin{tabular}{|c|}
\hline И к нему обычно ещё мисо сум отдельно подают \\
\hline Und dazu wird normalerweise noch eine Miso-Summe getrennt serviert \\
\hline Nachricht \#48 - 15.02.2018 - 15:56 \\
\hline
\end{tabular}

$\rightarrow$

\begin{tabular}{|c|}
\hline Не продают? \\
\hline Nicht verkauft? \\
\hline Nachricht \#49 - 15.02.2018 - 15:57 \\
\hline
\end{tabular}

$\rightarrow$

\begin{tabular}{|c|}
\hline Ааааа, их подают \\
\hline Achso, die werden serviert \\
\hline Nachricht \#50 - 15.02.2018 - 15:57 \\
\hline
\end{tabular}

\begin{tabular}{|c|}
\hline Ты меня прямо испытываешь на прочность \\
\hline Du stellst mich ja auf eine harte Probe \\
\hline Nachricht \#51 - 15.02.2018 - 15:57 \\
\hline
\end{tabular}

$\rightarrow$

\begin{tabular}{|c|}
\hline Мисо см? \\
\hline Miso-Summ? \\
\hline Nachricht \#52-15.02.2018 - 15:57 \\
\hline
\end{tabular}

$\rightarrow$

\begin{tabular}{|c|}
\hline Суп \\
\hline Suppe \\
\hline Nachricht $\# 53-15.02 .2018-15: 58$ \\
\hline
\end{tabular}

Dem in (178) dargestellten Ausschnitt geht $B$ 's Bericht über ihre Eindrücke von einem koreanischen Restaurant (vgl. (105)) voraus, wobei sie u.a. auch Gerichte 
erwähnt, die sie da gegessen hat (nicht abgebildet). Eine der Gerichtsbezeichnungen, und zwar Bibimbap, löst bei $A$ ein Bedeutungsverstehensproblem aus, woraufhin $B$ in \#47 und \#48 eine ausführliche Erklärung über dessen Zutaten sowie darüber, wie dieses gegessen wird, liefert. Die beiden Nachrichten, die als Reparaturdurchführungen für die Problemquelle in \#30 (nicht abgebildet) fungieren, enthalten jedoch selbst jeweils ein Reparandum, die von $A$ in \#49 und \#52 adressiert werden. Im ersten Fall stellt die Kombination aus einer Negationspartikel „ни“ und des Verbs „продают“ (,verkaufen-3P.PL‘) die Problemquelle dar, die in dem gegebenen Kontext keinen Sinn ergibt, was zu einer Fremdinitiierung in \#49 führt. Dabei wird die ursprüngliche Problemquelle „ни продают“ in der Initiierungsnachricht leicht modifiziert, indem die Negationspartikel „ни“ durch die andere, in dem Kontext korrekte, Negationspartikel „не“ ersetzt wird. Direkt in der nächsten Nachricht liefert $A$ jedoch selbst die Lösung für ihr Problem „их подают“ (,die werden serviert'), markiert mit dem iterierten Erkenntnisprozessmarker „Ааaаa“ (,achso“). Da der von $A$ vorgeschlagene Interpretationsvorschlag „их подают“ offensichtlich der richtige ist und das Problem somit gelöst ist, verzichtet $B$, die erst zwei Nachrichten später ,zum Wort kommt', darauf, diese Interpretation von $A$ zu bestätigen. Dieser Aufgabe zieht $B$ die Reparaturdurchführung zur zweiten Fremdinitiierung vor, die von $A$ in \#52 ebenfalls in Form der Teilwiederholung realisiert wurde. Diesmal bezieht sich die Fremdinitiierung aus \#52 auf die Problemquelle „мисо сум“ (мисо-су프 ,Miso-Suppe`) aus \#48. Der Vertipper wird von Bin \#53 korrigiert, indem sie nur die orthographisch korrekte Version der zweiten Konstituente verschickt. Dabei bleibt es unklar, ob sich die in \#52 abgebildete Teilwiederholung, die selbst einen Tippfehler in Form eines weggelassenen $y$ enthält, nur auf das Element „сум“ ( $с у \underline{\Pi}$,Suppe') richtete oder ob die Bedeutung des gesamten Ausdrucks „мисо сум“ (мисо-суп् ,Miso-Suppe`) erfragt wurde, da eigentlich die beiden Elemente wiederholt wurden. Immerhin scheint die Reparaturdurchführung in \#53 A zufrieden zu stellen, da sie keine erneute Fremdinitiierung unternimmt.

Ein Beispiel für eine Teilwiederholung in den deutschen Daten liegt in (179) vor. Hier formuliert Schreiberin $B$ in \#8 eine implizite Absage auf $A$ 's Frage nach ihrer Teilnahme an einem Basketballtraining. Dabei begründet sie ihre Entscheidung v.a. mit dem langen Weg sowie mit der Notwendigkeit bzw. Möglichkeit, diese Zeit effizienter zu nutzen, nämlich um zu arbeiten: 
(179) Auszug aus dem WhatsApp-Dialog \#3549:

Schreiberin $A$

Schreiberin $B$

$\rightarrow \quad \begin{array}{r}\begin{array}{r}\text { ja wäre glaube zu schwierig. bin vorher eh noch unterwegs. hab auch } \\ \text { keine lust } 3 \text { std zu fahren. da arbeite ich doch lieber. }\end{array} \\ \hline \text { Nachricht \#8-19.06.2014 - 15:45:05 }\end{array}$

$\rightarrow$

\begin{tabular}{|c|}
\hline arbeiten??? \\
\hline Nachricht \#9 $-19.06 .2014-15: 50: 05$ \\
\hline
\end{tabular}

$\rightarrow$

für die Uni. ist grad ziemlich viel.

Nachricht \#10 - 19.06.2014 - 15:55:05

$\rightarrow$

\begin{tabular}{|c|}
\hline Meinste quasi lernen?? \\
\hline Nachricht \#11 - 19.06.2014 - 16:00:05 \\
\hline
\end{tabular}

$\rightarrow$

ja und vorbereiten und so halt

Nachricht \#12 - 19.06.2014 - 16:05:05

Das von $B$ in \#8 erwähnte Verb „arbeite“ wird in $A$ 's Fremdinitiierung in Form einer leicht modifizierten Teilwiederholung aufgegriffen. Dabei könnte die Iteration der darauffolgenden Fragezeichen analog zur prominenten prosodischen Gestaltung einer Fremdinitiierung in der gesprochenen Sprache als ein Hinweis auf ein Erwartungsproblem gedeutet werden. Dieses könnte je nach geteiltem Wissen unterschiedlich bedingt sein. Beispielsweise kann bei $A$ ein Widerspruch entstehen, wenn sie das Verb arbeiten als ,einen Job haben“ interpretiert und weiß, dass $B$ zurzeit nur mit ihrem Studium beschäftigt ist und keinen Job hat. Alternativ könnte $B$ einen Job haben, aber sich gerade im Urlaub befinden oder von dem besprochenen Zeitpunkt abweichende Arbeitszeiten haben. Die von $B$ in \#10 gelieferte Präzisierung reicht $A$ nicht aus, sodass sie eine weitere Reparaturinitiierung in Form eines candidate understanding einleitet und das Verb lernen als Interpretationsvorschlag vorlegt, das von $B$ in der darauffolgenden Nachricht bestätigt wird.

Neben bereits veranschaulichten Teilwiederholungen findet sich im deutschen Subkorpus jeweils ein Dialog, in dem eine vollständige und eine als nicht abgeschlossen gestaltete Wiederholung als Fremdinitiierung vorliegen (vgl. Kap. 2.3.1.4). Auszug (180) präsentiert ein Beispiel für eine vollständige Wiederholung der problematischen Nachricht: 
(180) Auszug aus dem WhatsApp-Dialog \#2770:

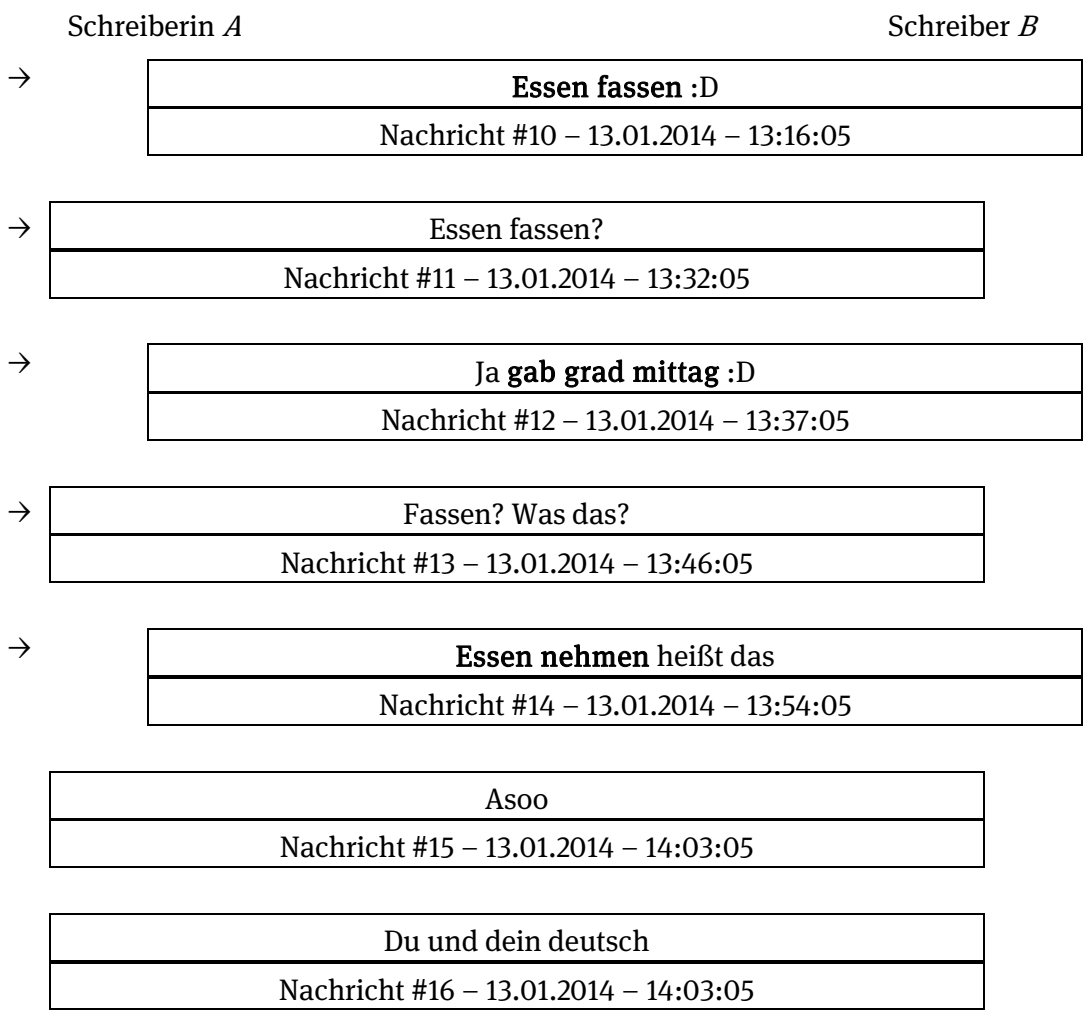

Während des Dialogs hält sich Schreiber $B$ an der Universität auf, während seine Freundin, Schreiberin $A$, zu Hause ist. In \#10 gibt $B$ im Telegrammstil einen ,Bericht' über seine aktuelle Tätigkeit durch. Die Kollokation „Essen fassen" aus \#10, die v.a. in der Soldatensprache gebräuchlich ist (vgl. https://www.duden.de/rechtschreibung/fassen), führt bei $A$ zu einem Bedeutungsverstehensproblem, das sie in Form einer (abgesehen von dem Emoticon) vollständigen Wiederholung andeutet. Diese Fremdinitiierung behandelt $B$ in \#12, indem er sie zuerst bestätigt und nachträglich die Erläuterung liefert, dass es gerade Mittagessen gab. Schreiberin $A$ gibt sich jedoch offensichtlich mit seiner Antwort nicht zufrieden, da sie zu einer Teilwiederholung wechselt, die lediglich die Problemquelle „Fassen?“ (\#13) aus der ihr offensichtlich nicht bekannten Kollokation beinhaltet. Außerdem sichert sie diese erneute Fremdinitiierung zusätzlich dadurch ab, dass sie eine zweite Reparaturinitiierung in Form eines elliptischen kopulativen Fragesatzes innerhalb derselben Nachricht nachschiebt (vgl. auch (45)). Diesmal liefert Schreiber $B$ ein Synonym zum ur- 
sprünglich problematischen Ausdruck und setzt somit das Verb „fassen“ (\#10) mit dem Verb „nehmen“ (\#14) gleich. Der kommunikative Erfolg der Reparatur wird von Schreiberin $A$ mit einem change-of-state token „Asoo“ (\#15) sowie dem nachträglichen ironischen Kommentar über B's Sprachvermögen quittiert (\#16).

Im Gegensatz zur vollständigen Wiederholung sowie Teilwiederholung zeichnet sich eine als unvollständig gestaltete Wiederholung in der gesprochenen Sprache durch die nicht-abgeschlossene Prosodie aus (vgl. Kendrick 2015: 173; vgl. Kap. 2.3.1.4). Graphisch kann diese Nicht-Abgeschlossenheit beispielsweise mit Auslassungspunkten dargestellt werden, wie in (181), in dem sich zwei Freundinnen über ihre Neujahrsvorsätze unterhalten:

(181) Auszug aus dem SMS-Dialog \#2030:

Schreiberin $A$

Schreiberin $B$

sowas wie: mehr für die uni tun, mehr sport, sich gesunder ernähren ...

:-D aber der einzige vorsatz, der bleibt, ist, die ferien bis zum 7.1. noch

ma richtig zu genießen $\wedge \wedge$

Nachricht \#5 - 02.01.2013-10:25

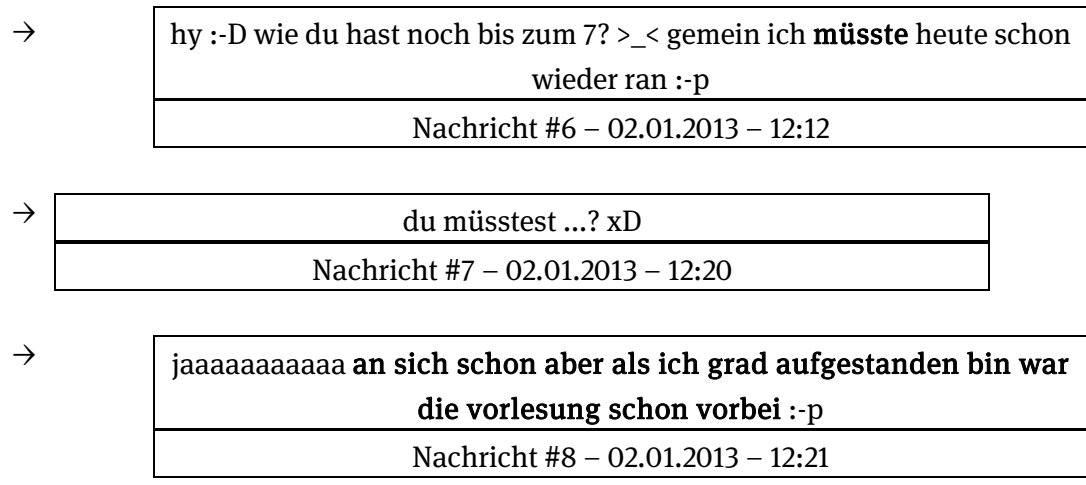

so startet man richtig ins neue jahr :-p wie gehts dir sonst so'? außer dass du ausgeschlafen sein musst, um diese uhrzeit? ${ }^{\wedge} \wedge$

Nachricht \#9 - 02.01.2013 - 12:21

Im Laufe des Austausches über die Neujahrsvorsätze hat sich herausgestellt, dass Schreiberin $A$ bis zum 7. Januar Ferien hat, während der Unterricht von Schreiberin $B$ schon am 2. Januar angefangen hat (vgl. \#6). Als Problemquelle fungiert dabei die Konjunktivform von müssen, die $B$ in \#6 benutzt und welche von $A$ im Rahmen einer Reparaturinitiierung in \#7 wiederaufgenommen wird. Darauf antwortet Schreiberin $B$, dass sie zwar heute eine Vorlesung hatte, diese 
aber verschlafen hat. Immerhin muss angemerkt werden, dass unvollständige Wiederholungen ähnlich wie Reparaturinitiierungen mit ,increments' (CouperKuhlen/Selting 2018: 178; vgl. Kap. 2.3.1.5) auch dazu beitragen können, die Progressivität der Interaktion durch die Ko-Konstruktion aufrechtzuerhalten (vgl. Couper-Kuhlen/Selting 2018: 183).

\subsubsection{Fremdinitiierungen in Form von copular interrogative clauses}

Im deutschen Subkorpus finden sich insgesamt 17 Fälle von copular interrogative clauses mit den Fragewörtern wer, was, wann und wo. Dabei kann als Prädikativ sowohl das Demonstrativpronomen das (182) als auch die wiederholte Problemquelle (183) fungieren. Manchmal wird der Frage die Modalpartikel denn hinzugefügt. Mit den in den Daten vorkommenden copular interrogative clauses werden einerseits nicht-eindeutige Referenzausdrücke (vgl. (183)) problematisiert, die „nicht korrekt auf den Adressaten zugeschnitten wurden“ (Golato 2013: 47). Andererseits werden copular interrogative clauses auch bei Bedeutungsverstehensproblemen eingesetzt, wie in (182) (vgl. dazu auch Beispiel (42) aus der gesprochenen Sprache):

(182) Auszug aus dem WhatsApp-Dialog \#3393:

Schreiberin $A$ Schreiberin $B$

\begin{tabular}{|c|}
\hline Ich hab einen film für uns gefunden !!! Im kino \\
\hline Nachricht \#3 - 27.04.2014 - 16:40:05 \\
\hline
\end{tabular}

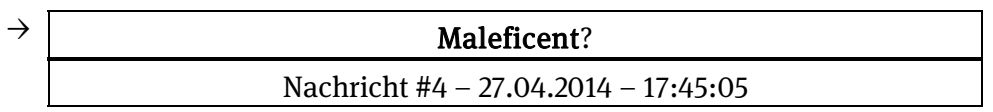

\begin{tabular}{l}
$\rightarrow \quad$ Was ist das denn ${ }^{\prime}$ :D Ne schöne und das biest mit hugh $<3$ \\
\hline Nachricht \#5 - 27.04.2014 - 17:46:05 \\
\hline Oh geil wann? \\
\hline Nachricht \#6 - 27.04.2014 - 18:34:05 \\
\hline Ist übrigens dornröschen mit Angelina Jolie als böse fee \\
\hline Nachricht \#7 - 27.04.2014 - 18:36:05
\end{tabular}

Mhh :/ Ab 1.5. :)

Nachricht \#8-27.04.2014 - 18:52:05 
In (182) kündigt Schreiberin $B$ ihrer Schwester an, dass sie einen für die beiden interessanten Film gefunden hat. Daraufhin versucht $A$ zu raten, um welchen Film es gehen könnte, und vermutet in \#4, dass $B$ den Film „Maleficent“ meint. Diese Vermutung wird von $B$ im zweiten Teil der Nachricht \#5 aber abgelehnt. Der erste Teil der Nachricht wird dabei für eine Fremdinitiierung in Form eines copular interrogative clause reserviert. Mithilfe dieser Reparaturinitiierung gibt $B$ ihrer Interaktionspartnerin zu erkennen, dass sie den von $A$ gemeinten Film nicht kennt. Das ,weit lächelnde“ Emoticon mit einem ,Schweißtropfen“ „: $D^{\prime \prime}$ könnte man in diesem Fall als eine face work-Strategie interpretieren, mit der $B$ die Peinlichkeit ihres Unwissens mit einem gewissen Grad der Selbstironie reflektiert. Obwohl zumindest in der gesprochenen Sprache die Tendenz besteht, ein Problem so schnell wie möglich zu lösen (vgl. Schegloff 1979, 2007; Heritage 2007; Pfeiffer 2017a), gibt Schreiberin $A$ in dem Fall einer anderen sprachlichen Handlung den Vorrang, bevor sie zur Reparaturdurchführung (\#7) übergeht: Sie gibt zunächst eine Bewertung zum von $B$ vorgeschlagenen Film Schöne und das Biest ab sowie fragt, wann dieser gezeigt wird. Erst danach liefert $A$ eine kurze Beschreibung zum problematischen Filmtitel, indem sie den Film mit einem bekannten Märchen Dornröschen in Verbindung bringt sowie die Hauptdarstellerin nennt. Das im Mittelfeld verwendete „übrigens“ (\#7) soll dabei vermutlich eine Verbindung zu einer zurückliegenden Reparaturinitiierung herstellen (zur Funktionen von übrigens vgl. Egbert 2003). Die Tatsache, dass zwei weitere sprachliche Handlungen vor einer Reparaturdurchführung vollzogen werden, kann unterschiedlich motiviert werden. Zum einen kann die gewählte Reihenfolge mit der Länge bzw. dem Formulierungsaufwand der Nachrichten \#6 und \#7 zusammenhängen. Zum anderen wird dadurch der Film Schöne und das Biest als thematischer Schwerpunkt der Interaktion im Vergleich zur Reparatursequenz implizit als relevanter eingestuft, da diese einen ,punktuellen' Charakter hat und keine schwerwiegende Hindernisse für die Entfaltung des ,Hauptthemas' des Dialogs schaffen würde. Die Reparaturdurchführung in \#7 liegt dabei also im after next opportunity space. Es sei allerdings angemerkt, dass fremdinitiierte Selbstreparaturen in dieser Position - genauso wie im Falle von selbstinitiierten Selbstreparaturen - im Vergleich zu Reparaturen im next opportunity space seltener vorkommen (jeweils acht Fälle in den beiden Teilkorpora; für das Russische vgl. (104)). Dies zeigt die Orientierung der Schreiber an der möglichst schnellen Bearbeitung der vom Rezipienten gelieferten Fremdinitiierung (für die gesprochene Sprache vgl. Schegloff 1979, 2007; Heritage 2007; Pfeiffer 2017a), wofür sich next opportunity space als die erste mögliche Position anbietet. 
Ein Beispiel, in dem ein copular interrogative clause auf ein vorliegendes Referenzproblem (eine nicht-eindeutige Ortsreferenz) gerichtet ist, liegt in Ausschnitt (183) vor, der aus einem Nachrichtenaustausch zwischen Schreiber $A$ und Schreiberin $B$ stammt, die ein Pärchen sind:

(183) Auszug aus dem Dialog \#3710:

Schreiber $A$

Schreiberin $B$

$\rightarrow$

\begin{tabular}{|c|}
\hline Ich lass das hadny hier. Wenn was wichtiges ist schreib NAME! \\
\hline Nachricht \#3 - 15.11.2014- 12:48:26 \\
\hline
\end{tabular}

$\rightarrow$

Wo ist "hier"?

Nachricht \#4 - 15.11.2014 - 12:49:04

$\rightarrow$

\begin{tabular}{|c|}
\hline In der alten wohnung \\
\hline Nachricht \#5 - 15.11.2014 - 12:49:17 \\
\hline
\end{tabular}

Ach hwrje kannst ja nicht wissen

Nachricht \#6 - 15.11.2014 - 12:49:34

\begin{tabular}{|c|}
\hline Wir gehen wieder rueber \\
\hline Nachricht \#7 - 15.11.2014 - 12:49:56 \\
\hline
\end{tabular}

\begin{tabular}{|c|}
\hline Okay! Ja das dachte ich mir schon! \\
\hline Nachricht \#8 - 15.11.2014 - 12:50:27 \\
\hline
\end{tabular}

In Nachricht \#3 benutzt Schreiber $A$ das deiktische Adverb „hier“, welches sich im weiteren Verlauf der Kommunikation jedoch als problematisch erweist. Daher initiiert Schreiberin $B$ in \#4 eine Reparatur, indem sie das Interrogativadverb „wo“ mit einem wiederholten Reparandum aus \#3 mithilfe des Kopulaverbs verbindet. Dabei markiert sie die wiederholte Problemquelle zusätzlich mit Anführungszeichen, sodass diese zum einen graphisch hervorgehoben und zum anderen als Zitat aus einer der vorherigen Nachrichten kenntlich gemacht wird (vgl. Mostovaia 2018). Eine für das vorliegende Referenzproblem typische Reparaturdurchführung erfolgt in \#5, indem ein präziserer Referenzausdruck geliefert wird - in dem Fall „In der alten wohnung“.

Im russischen Subkorpus können den copular interrogative clauses mit der Struktur Fragewort + Kopulaverb + X? Fragen im Format это (,das $)+$ Frage- 
wort? bzw. Fragewort + это (,das ${ }^{\circ}$ ? $^{137}$ gegenübergestellt werden, da es im Russischen zwar das Kopulaverb есть (,sein`) gibt, dieses jedoch im Präsens i.d.R. nicht realisiert wird. Dabei fungiert das deiktische Pronomen это (,das`) als eine obligatorische Komponente der Frage (vgl. (184)), während die Wiederaufnahme der Problemquelle fakultativ ist und oft in der Form einer Linksversetzung realisiert wird (vgl. (185)). In den russischen Daten schließt dieses Format Interrogativa wie что (,was'), где (,wо'), как (,wie') und во сколько (,um wie viel, wann') ein (neun Tokens).

In (184), das ähnlich wie (182) aus dem deutschen Korpus funktioniert, wird ein Bedeutungsverstehensproblem thematisiert (vgl. денотативный запрос (,Frage zur Denotation`) in Goletiani 2003: 195-196):

(184) Auszug aus dem SMS-Dialog \#2527:

Schreiberin $A$

Schreiberin $B$

$\rightarrow$

Слушай, я что подумала А может мы в сквош сходим поиграть? :-)

Weißt du, was ich mir überlegt habe. Lass uns mal Squash spielen gehen. :-)

Nachricht \#1 - 22.07.2013 - 16:05:05

$\rightarrow$

\begin{tabular}{|c|}
\hline А что это? \\
\hline Was ist das denn? \\
\hline Nachricht \#2 - 22.07.2013 - 16:06:05 \\
\hline
\end{tabular}

$\rightarrow$ Google в помощь :-) Как теннис, только игроки на одной стороне и играют со стенкой)

Frag mal Google :-) Wie Tennis, aber die Spieler stehen auf der gleichen Seite des Spielfeldes und schlagen den Ball gegen die Wand)

Nachricht \#3 - 22.07.2013 - 16:08:05

Bei der Vereinbarung eines Treffens schlägt Schreiberin $A$ ihrer Freundin vor, Squash spielen zu gehen. Im Rahmen einer Reparaturinitiierung in Nachricht \#2 fordert Schreiberin $B$ nähere Informationen zur Bedeutung des Wortes „сквош“ (,Squash') ein. Darauf reagiert Schreiberin $A$ zuerst mit einem ironischen Ratschlag, dass Schreiberin $B$ die Google-Suchmaschine zu Rate ziehen sollte. Die-

137 Die Frage, ob die Reihenfolge des Pronomens und des Fragewortes einen funktionalen Unterschied hervorruft, muss in zukünftigen Untersuchungen geklärt werden. 
sen versieht sie mit dem Emoticon ,:-)“, welches in dem Fall eine für $B$ gesichtsbedrohende Handlung modalisieren und als einen Witz rahmen soll (vgl. Pappert 2017: 195-196). Danach liefert $A$ dennoch selbst eine Definition, in der sie das Spiel mit Tennis (also mit einer ihrer Meinung nach anscheinend Schreiberin $B$ vertrauten Sportart) vergleicht und durch den Gebrauch der Partikel „только“ (,nur`) die Unterschiede zwischen den beiden Spielen herausstellt.

Die Reparaturinitiierung in (185) stellt ein Kontrastbeispiel zu der Reparatur aus dem deutschen Dialog (183) dar, in dem ebenfalls ein deiktischer Ausdruck, der sich auf eine Ortsreferenz bezieht, als Problemquelle fungiert:

(185) Auszug aus dem Viber-Dialog \#3168:

Schreiberin $A$

Schreiberin $B$

\begin{tabular}{|c|}
\hline Ты где? \\
\hline Wo bist du? \\
\hline Nachricht \#5 $-10.03 .2014-14: 05: 05^{138}$ \\
\hline
\end{tabular}

$\rightarrow$

\begin{tabular}{|c|}
\hline Тута уже \\
\hline Ich bin schon hier \\
\hline Nachricht \#6 -10.03.2014-14:05:05 \\
\hline
\end{tabular}

$\rightarrow$

\begin{tabular}{|c|}
\hline Тута это где?? \\
\hline Wo ist hier?? \\
\hline Nachricht \#7 - 10.03.2014 - 14:05:05 \\
\hline
\end{tabular}

\begin{tabular}{|c|}
\hline Я в корпус зашла \\
\hline Ich bin in das Gebäude reingegangen \\
\hline Nachricht \#8 - 10.03.2014 - 14:05:05 \\
\hline
\end{tabular}

$\rightarrow$

\begin{tabular}{|c|}
\hline B 4 кopпyce \\
\hline Im Gebäude 4 \\
\hline Nachricht \#9-10.03.2014-14:05:05 \\
\hline
\end{tabular}

Bei (185) handelt es sich um einen Auszug aus einem Dialog über den Messenger-Dienst Viber, der zwischen zwei Freundinnen erfolgt, die auf dem Weg zu einem gemeinsamen Treffpunkt sind. In Nachricht \#5 fragt Schreiberin $B$ ihre Freundin nach ihrem Standort, woraufhin Schreiberin $A$ antwortet, dass sie

138 Vgl. Fußnote 117. 
bereits ,hier“ („Тута уже“ in \#6) ist. Analog zu (183) stellt das Adverb „Тута“ (\#6) - eine umgangssprachliche Variante von тут (,hier`) - in diesem Fall eine Problemquelle dar, die in der darauffolgenden Fremdinitiierung von Schreiberin $B$ thematisiert wird. Dabei wird die wiederholte Problemquelle „Тута“ (,hier') anaphorisch mit dem Demonstrativpronomen „это“ (,das') aufgenommen, während das Interrogativadverb „где“ (,шо) an die letzte Stelle rückt, sodass man in dem Fall von einer Linksversetzung ausgehen könnte (vgl. ähnliche Fälle für что за X-Konstruktion in Podlesskaya 2007). Im Gegensatz zu (183) enthält diese Reparaturinitiierung außerdem kein Kopulaverb und keine Anführungszeichen für die Markierung der wiederholten Problemquelle. Als Reparaturdurchführung liefert $A$ in \#9 einen vollständigen referenziellen Ausdruck, nämlich die Nummer eines universitären Gebäudes.

\subsubsection{Fremdinitiierungen mit candidate solutions}

Candidate solutions gehören sowohl im russischen als auch im deutschen Subkorpus mit jeweils 16 und 79 Fällen (oder in relativen Zahlen mit jeweils 19\% und 29\% aller Fremdinitiierungen) zu dem am häufigsten benutzten Initiierungsformat. Das könnte damit zusammenhängen, dass candidate solutions auf dem von Schegloff/Jefferson/Sacks (1977) aufgespannten Kontinuum ,schwache - starke Reparaturinitiierungen“ als ein relativ ,starkes' Initiierungsformat angesehen werden und somit das Reparandum genau lokalisieren sowie gleichzeitig die Art des vorliegenden Problems offenlegen. Wenn man candidate solutions mit anderen Initiierungsformaten hinsichtlich des Aufwandes vergleicht, der bei der Produktion der jeweiligen Reparaturinitiierung entsteht, stellt man fest, dass candidate solutions, die meist als elliptische oder vollständige Sätze realisiert werden, beispielsweise im Gegensatz zu Fremdinitiierungen mit Fragewörtern mehr Zeit für deren Produktion benötigen. Nach der Häufigkeit von candidate solutions im jeweiligen Teilkorpus $\mathrm{zu}$ urteilen, scheint jedoch die präzise Lokalisierung der Problemquelle, die vom Produzenten der Problemquelle meist lediglich bestätigt werden muss, den Vorrang vor der schnellen Produktion der Beiträge zu haben.

Sowohl in den russischen als auch in den deutschen Daten kommen candidate solutions meist in Form von Konstituenten vor, die syntaktisch entweder einen (i.d.R. deiktischen) Ausdruck aus der problematischen Nachricht ersetzen (vgl. (186)) oder die ursprüngliche Nachricht semantisch und syntaktisch erweitern (vgl. (187)): 
(186) Auszug aus dem WhatsApp-Dialog \#4352:

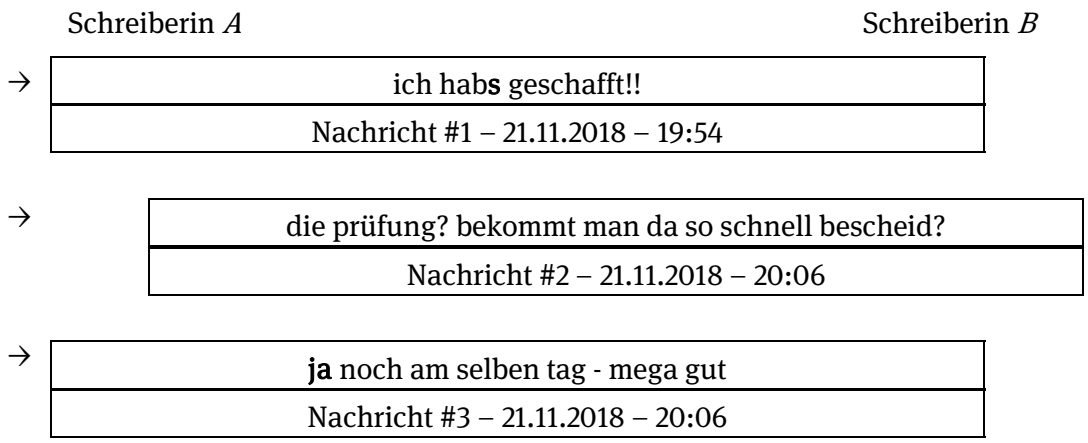

In (186) problematisiert $B$ das als Enklitikon realisierte Pronomen es aus dem Beitrag ihrer Tochter (vgl. \#1) und schlägt in \#2 die Nominalphrase „die prüfung“ als candidate reference dafür vor. Das Fragezeichen, das der von $B$ vermuteten Referenz folgt, weist auf die Notwendigkeit der Ratifikation seitens Schreiberin $A$ hin. Diese erfolgt implizit in \#3, indem $A$ die zweite Frage ihrer Mutter nach der Frist für die Bekanntgabe der Note bestätigt.

Ein weiteres Beispiel für eine candidate reference stellt der russischsprachige Auszug (187) bereit, in dem sich Schreiber $A$ bei seiner Freundin erkundigt, ob sie über den bevorstehenden Poesieabend informiert wurde:

(187) Auszug aus dem Dialog \#3024:

\begin{tabular}{l} 
Schreiber $A$ \\
$\rightarrow$\begin{tabular}{c|} 
[Женское имя], а вам [фамилия преподавателя]говорила про \\
поэтический вечер?
\end{tabular} \\
\hline $\begin{array}{c}\text { [Frauenname], hat [Name der Dozentin] euch über den Poesieabend } \\
\text { Bescheid gesagt? }\end{array}$ \\
\hline Nachricht \#1 - 11.02.2014 - 14:05:05 \\
\hline Который завтра? \\
\hline Über den, der morgen stattfindet? \\
\hline Nachricht \#2 - 11.02.2014 - 14:07:05 \\
\hline Ara, ты пойдешь? \\
\hline Jap, gehst du hin? \\
\hline Nachricht \#3 - 11.02.2014 - 14:09:05 \\
\hline
\end{tabular}


Im Gegensatz zu (186) stellt die problematische Referenz „поэтический вечер“ (,Poesieabend') zwar keinen deiktischen Ausdruck dar, muss aber dennoch im Rahmen einer Reparatur bearbeitet werden. Die von $B$ in \#2 angebotene candidate reference in Form eines elliptischen Relativsatzes fungiert nicht nur als Fremdinitiierung, sondern auch als eine implizite Bestätigung der in \#1 von $A$ gestellten Frage. Zudem lässt sich die candidate reference „Который завтра?“ (,Über den, der morgen stattfindet?`) als syntaktische Fortsetzung von \#1 betrachten, wodurch die Problemquelle, die einer Präzisierung bedarf, genauer lokalisiert wird. Die Reparaturdurchführung erfolgt in \#3 in Form einer Bestätigung der von $B$ vorgelegten candidate reference mit der Interjektion „Ага“ (,ja/jap').

Im deutschen Subkorpus sind candidate solutions überwiegend mit elliptischen sowie syntaktisch vollständigen Entscheidungsfragen vertreten. Bei einigen von ihnen lassen sich gewisse Muster erkennen, die sich v.a. im Gebrauch einzelner Lexeme wie also (sechs Fälle), heißen (sechs Fälle) und meinen (13 Fälle) niederschlagen. Darüber hinaus kommen in den untersuchten Kurznachrichtendialogen neben Entscheidungsfragen auch Alternativfragen (13 Fälle) vor, die syntaktisch sowohl vollständig als auch elliptisch realisiert werden. In den russischen Daten finden sich allerdings abgesehen von einer auf zwei Nachrichten gesplitteten Alternativfrage ausschließlich Entscheidungsfragen, die aber leider kaum rekurrente Reparaturmarker aufweisen. Einen reparativen Charakter kann man lediglich dem Lexem так (,so/auf solche Art und Weise‘) sowie der abgekürzten Konjunktion T.e. (,d.h.') zuschreiben.

Bei sechs candidate solutions in den deutschen Daten lässt sich die Verwendung von also „zur Anzeige einer intersubjektiven Inferenz“ feststellen (Deppermann/Helmer 2013: 5). Dabei wird also in den Kurznachrichten im Gegensatz zur gesprochenen Sprache ausschließlich mit Phrasen und nicht mit ganzen Sätzen kombiniert (vgl. Deppermann/Helmer 2013: 11-13, die diese beiden Optionen beschreiben). Zudem enthalten die untersuchten Fremdinitiierungen im Unterschied zu den Ergebnissen von Deppermann/Helmer (2013: 1516) keine Vorlaufelemente, was vermutlich daran liegt, dass diese auch in den gesprochensprachlichen Daten zum einen relativ selten vorkommen (lediglich in 28 von 157 Fällen) und zum anderen v.a. mit den Rückversicherungssignalen vertreten sind, die in der schriftbasierten Kommunikation anders eingesetzt werden. Eine Fremdinitiierung mit also wird mit Auszug (188) veranschaulicht, in dem Schreiberin $A$ ihrem Freund über ihren Traum berichtet: 
(188) Auszug aus dem WhatsApp-Dialog \#3281:

Schreiberin $A$

Schreiber $B$

Einen wunderschönen guten Morgen 6 Heute ist ja schon der dritte, sollen wir dann heut Abend in den Zirkus? :-)

Nachricht \#1 - 03.01.2014 - 09:11:00

$\rightarrow$\begin{tabular}{c|} 
Morgeen mein Schatz :-) hab vom Skifahren geträumt :D war aber \\
so cool und bin immer nur mit einem Brett gefahren \\
wir machen Ja können
\end{tabular}

$\rightarrow \quad$ Also Snowboard? $\Leftrightarrow$

$\rightarrow \mid$\begin{tabular}{|c|}
\hline Nee nur auf einem Skier, auch nur mit einem Stock :D \\
\hline Nachricht \#4 - 03.01.2014 - 10:43:00 \\
\hline
\end{tabular}

\begin{tabular}{|c|}
\hline Boa richtiger Rebell 6 (3) \\
\hline Nachricht \#5 - 03.01.2014 - 11:17:00 \\
\hline
\end{tabular}

Bei der Beschreibung ihres Traums benutzt $A$ den Ausdruck „mit einem Brett gefahren“ (Nachricht \#2), aus dem $B$ offensichtlich inferiert, dass es sich um ein Snowboard handelt. Sein candidate understanding präsentiert $B$ in Nachricht \#3 in Kombination mit einem ,lächelnden' Emoji. Während die meisten candidate understandings in den analysierten Daten bestätigt werden, wird $B$ 's Verstehensversuch von $A$ abgelehnt und korrigiert (vgl. \#4).

Des Weiteren finden sich in den deutschen Daten sechs Reparaturinitiierungen mit dem Verb heißen. Dabei nehmen diese zum einen die Form einer Ergänzungsfrage „was soll das heißen??“ (\#3510 in der MoCoDa) an (vgl. Deppermann/Schmitt 2008: 226-227), zum anderen werden Fremdinitiierungen mit heißen als Entscheidungsfragen realisiert, und zwar im Format (das) + heißt + (Problemquelle) + candidate solution?, wo fakultative Elemente eingeklammert sind (vgl. Deppermann/Schmidt 2014; Kaiser 2016; Helmer/Zinken 2019). Eine elliptische Version der Reparaturinitiierung in Form einer Entscheidungsfrage mit heißen, in der sowohl das Reparandum als auch das Demonstrativpronomen weggelassen werden, liegt in (189) vor: 
(189) Auszug aus dem WhatsApp-Dialog \#3663:

Schreiberin $A$

Schreiberin $B$

\begin{tabular}{|c|}
\hline NAME? Wegen Gitarre. Ab wann kannst du jetzt? \\
\hline Nachricht \#1 - 05.09.2014 - 11:49 \\
\hline
\end{tabular}

\begin{tabular}{|c|}
\hline NAME sagt wir können um 3 kommen \\
\hline Nachricht \#2 - 05.09.2014 - 11:53 \\
\hline
\end{tabular}

\begin{tabular}{|c|}
\hline Ok \\
\hline Nachricht \#3 $-05.09 .2014-11: 54$ \\
\hline
\end{tabular}

\begin{tabular}{|c|}
\hline Passt \\
\hline Nachricht \#4-05.09.2014-11:54 \\
\hline
\end{tabular}

\begin{tabular}{|c|}
\hline Super :) für in Zukunft auch oder nur heute? \\
\hline Nachricht \#5 - 05.09.2014-11:54 \\
\hline
\end{tabular}

$\rightarrow$

\begin{tabular}{|c|}
\hline :) \\
\hline Nachricht \#6-05.09.2014-11:55 \\
\hline
\end{tabular}

$\rightarrow$

\begin{tabular}{|c|}
\hline Heißt ja? \\
\hline Nachricht \#7 $-05.09 .2014-11: 55$ \\
\hline
\end{tabular}

$\rightarrow$

\begin{tabular}{|c|}
\hline :D \\
\hline Nachricht \#8 - 05.09.2014-11:55 \\
\hline
\end{tabular}

$\rightarrow$

Für heute.

Nachricht \#9 - 05.09.2014 - 11:57

Zwei Schwestern machen per WhatsApp einen Termin aus. Nachdem der Termin feststeht, fragt Schreiberin $A$ in Nachricht \#5, ob die eben festgelegte Zeit nur für heute oder auch für später gilt. Das dieser Frage folgende Emoticon „:)“ (\#6) könnte dabei entweder als Fortsetzung B's vorheriger Nachricht (\#4, vgl. auch \#7 und \#8 im gleichen Dialog) oder als ihre Reaktion auf $A$ 's Frage (\#5) interpretiert werden. Von $A$ wird es als Antwort auf ihre Frage gedeutet, die sie allerdings nicht ganz versteht und zu der sie daher in \#7 eine Reparatur initiiert, in der sie ein Ja als candidate understanding anbietet. Allerdings muss angemerkt werden, dass die Fremdinitiierung ebenfalls potenziell problematisch ist, 
da das von $A$ vorgelegte Ja als eine Antwort auf eine Alternativfrage mit zwei einander ausschließenden Optionen eingesetzt wird. Vermutlich bezieht sich dabei die bestätigende Antwort auf eine von $A$ bevorzugte Option, die jedoch aus dem Kontext - zumindest aus der Beobachterperspektive - nicht ersichtlich ist. Bei der Reparaturdurchführung orientiert sich $B$ allerdings nicht an der vorliegenden Reparaturinitiierung, die eine Bestätigung oder eine Ablehnung des vorgelegten candidate understanding ,ja“ (\#7) voraussetzt, sondern an der der Problemquelle vorangehenden Alternativfrage und bestätigt dann mithilfe einer Wiederholung eine der in \#5 vorgegebenen Optionen, nämlich die Zeitreferenz „heute“.

Eine der Entsprechungen für das heißt im Russischen stellt die Konjunktion то есть dar, die ebenso wie ihr deutsches Pendant auch bei selbstinitiierten Selbstreparaturen zum Einsatz kommt (für das Russische vgl. Podlesskaya 2014; für das Deutsche vgl. Kaiser 2016; zu selbstinitiierten Selbstreparaturen mit то есть vgl. (95)):

(190) Auszug aus dem SMS-Dialog \#3170:

Schreiberin $A$

Schreiberin $B$

\begin{tabular}{|c|}
\hline Ну что. Как дела на подготовке в фирме? \\
\hline Und? Wie läuft dein Betriebspraktikum bei der Firma? \\
\hline Nachricht \#1 - 10.03.2014 - 14:05:05 ${ }^{139}$ \\
\hline
\end{tabular}

$\rightarrow \begin{gathered}\text { Привет)все хорошо, я только вчера сходила на стажировку сегодня } \\ \text { уже не поехала))))потому что это просто риэлтор и я сидела } \\ \text { обзванивала все варианты квартир полдня }\end{gathered}$

Hallo) Alles ist gut, ich bin nur gestern dahingefahren und heute nicht mehr)))) weil es nur ein Immobilienmakler ist und ich einen halben Tag am Telefon gesessen habe und wegen aller in Frage kommenden Wohnungsangebote telefoniert habe

Nachricht \#2 - 10.03.2014 - 14:05:05

$\rightarrow$

Aaа. T.e. не было тех лекций и тп? Вот это облом((((

Achso, d.h. es gab diese Vorlesungen usw. nicht? Das ist aber sehr schade $((()$

Nachricht \#3 - 10.03.2014 - 14:05:05

139 Vgl. Fußnote 117. 
$\rightarrow$

Ну типа первый этап это обзвон параллельно с лекциями

Ja, auf der ersten Etappe sollten wohl Vorlesungen mit den

Telefonaten gekoppelt werden

Nachricht \#4 - 10.03.2014 - 14:05:05

In (190) erkundigt sich $B$ bei ihrer Bekannten, wie ihr Betriebspraktikum verläuft. Darauf antwortet $A$, dass sie die Stelle aufgegeben hat, da diese ihren Vorstellungen von einem Praktikum nicht entspricht, weil es sich ergeben hat, dass sie lediglich Telefonate für einen Immobilienmakler führen sollte. Nach$\operatorname{dem} A$ über das Scheitern ihres Praktikums berichtet hat, quittiert Schreiberin $B$ die erhaltene Information mit dem Erkenntnisprozessmarker „Aaa“ (,achso) und schildert unmittelbar danach ihr Erwartungsproblem. Dabei fragt sie nach der von ihr vorausgesetzten und offensichtlich den beiden bekannten Aktivität, nämlich „тех лекций“ (wortwörtlich ,jene Vorlesungen'), die im Rahmen von $A$ 's erstem Tag stattfinden sollte. $A$ reagiert auf die von $B$ in \#3 gelieferte Annahme über die nicht-stattgefundenen Vorlesungen, indem sie ihr mitteilt, dass die Vorlesungen parallel zu den Telefonaten verlaufen sollten.

Neben Fremdinitiierungen mit heißen und alsoliegen in den deutschen Daten dreizehn candidate understandings vor, in denen das Verb meinen auftritt. Dabei werden diese Reparaturinitiierungen meist in Form von Entscheidungsfragen (Meinst du + candidate solution?) realisiert, während die umgekehrte Reihenfolge der Konstituenten candidate solution + meinst du?, die von Egbert (2009: 170) für Fremdinitiierungen in der gesprochenen Sprache belegt wurde, lediglich in einem Fall vorkommt (vgl. Dialog \#4249 in der MoCoDa).

In den meisten Reparaturinitiierungen wird meinen im Präsens verwendet, nur in einem Beispiel findet sich eine präteritale Form. Bei der Verwendung im Präsens taucht meinen dreimal in der klitisierten Form meinste auf. Neben Fragesätzen werden Fremdinitiierungen mit meinen vereinzelt auch in Form von Deklarativsätzen eingesetzt: Einer von vier solchen Fällen stellt einen asyndetischen untergeordneten Satz dar, der im weiteren Verlauf der Arbeit im Kontext von candidate corrections ausführlich diskutiert wird (vgl. (192)). Zunächst soll aber eine Fremdinitiierung mit meinen in Form einer Entscheidungsfrage im Format Meinst du + candidate solution? anhand von (191) veranschaulicht werden:

(191) Auszug aus dem WhatsApp-Dialog \#2798:

Schreiberin $A$

Schreiberin $B$

\begin{tabular}{|c|c|}
\hline \multirow[t]{2}{*}{$\rightarrow$} & Dein Foto ist voll schön Schatz \\
\hline & Nachricht \#1 - 19.01.2014 - 16:44:05 \\
\hline
\end{tabular}




\begin{tabular}{l}
$\rightarrow \quad$ Danke! Meinst du das Profilbild? \\
\cline { 2 - 2 }
\end{tabular}

Nachdem Schreiberin $A$ in \#1 eine positive Bewertung zu $B$ 's Foto abgibt, bedankt sich $B$ bei ihr dafür und präzisiert anschließend mithilfe einer candidate reference, die eine V1-Frage mit dem Verb meinen darstellt, auf welches Foto sich $A$ 's Nachricht bezieht (zu meinen vgl. Imo 2007: 173-200). $A$ reagiert mit einer für diese Konstellation typischen Reparaturdurchführung, indem sie die vorgelegte candidate solution bestätigt.

Wie bereits angekündigt, soll nun in (192) eine candidate correction in Form einer Deklarativsatzfrage mit meinen vorgestellt und mit einer syntaktisch ähnlich aufgebauten fremdinitiierten Fremdreparatur aus (193) kontrastiert werden. Dabei liegt in (192) eine Fremdinitiierung vor, die mit Problemen der Akzeptabilität zu tun hat, d.h. sie spricht solche Elemente an, die aus der Perspektive des Rezipienten der Problemquelle im weiten Sinne nicht ,korrekt' sind (vgl. Svennevig 2008). Dadurch haben Fremdinitiierungen in Form von candidate corrections einige Berührungspunkte mit fremdinitiierten Fremdreparaturen, sodass die Grenzen gelegentlich fließend sind:

(192) Auszug aus dem SMS-Dialog \#4181:

Schreiberin $A$

Schreiber $B$

$\rightarrow$

Hey, hab eine Frage hast du zufällig Zuhause Zitronensäure? Wollte knete für Kindergarten machen. Bist du da. Hey?

Nachricht \#1 - 24.10.2016 - 18:10:17

\begin{tabular}{|c|}
\hline $\begin{array}{l}\text { Guten Tag, Alexandra! Wie geht es dir? Mir geht es gut! Hattet ihr } \\
\text { gestern Abend noch viel Spaß? Ich glaube, du meinst Zitronensaft, } \\
\text { oder? Zitronensäure ist ätzend und gefährlich! }\end{array}$ \\
\hline Nachricht \#2 - 24.10.2016 - 18:15:23 \\
\hline Ja Zitronensaft. Ja den hatten wir gestern \\
\hline Nachricht \#3 - 24.10.2016 - 18:15:46 \\
\hline $\begin{array}{l}\text { Ja, ich müsste eigentlich noch eine Flasche haben, aber ob diese noch } \\
\text { voll ist, weiß ich nicht. }\end{array}$ \\
\hline Nachricht \#4 - 24.10.2016 - 18:21:32 \\
\hline
\end{tabular}


In (192) legt Schreiber $B$ in \#2 eine candidate correction vor, indem er die von $A$ erfragte Zitronensäure durch eine Bezeichnung der für $A$ 's Zwecke passenderen Flüssigkeit, nämlich den Zitronensaft, ersetzt, wobei der Unterschied zwischen den beiden Flüssigkeiten anschließend aufgezeigt wird. Dabei lässt sich „Ich glaube, du meinst Zitronensaft, oder?“ (\#2) v.a. dank dem Vergewisserungssignal „oder“ und dem Fragezeichen eher als eine candidate correction und nicht als eine Fremdreparatur interpretieren, da dies die Bestätigung der vorgelegten Interpretation durch Schreiberin $A$, die in \#3 erfolgt, relevant macht.

Im Gegensatz zu (192) lässt sich die Reparatur in (193) eher als eine fremdinitiierte Fremdreparatur einstufen, obwohl die beiden viele Gemeinsamkeiten aufweisen:

(193) SMS-Dialog \#1445:

Schreiber $A$

Schreiberin $B$

\begin{tabular}{|c|}
\hline Und? \\
\hline Nachricht \#1 - 15.11.2011 - 10:12:05 \\
\hline
\end{tabular}

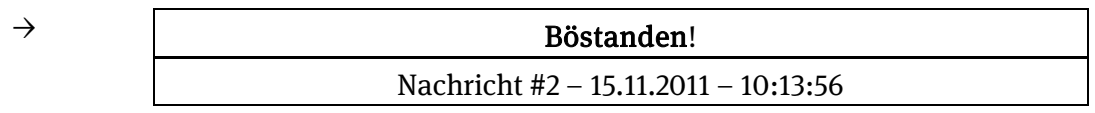

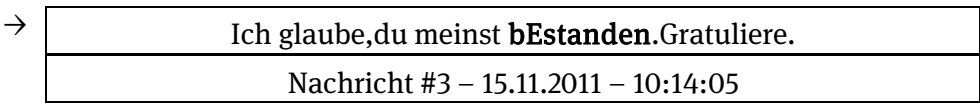

$\rightarrow$

Nein du klugscheißer.ich meine böstanden,das ist lautsprache und saucool.danke.und weils so schön ist:lol,omfg,grins,liebguck,wtf,rofl und all der andere quatsch

Nachricht \#4 - 15.11.2011 - 10:16:05

\begin{tabular}{|c|}
\hline ok.... \\
\hline Nachricht \#5 - 15.11.2011 - 10:18:05 \\
\hline
\end{tabular}

Die Problemquelle liegt in B's Mitteilung (\#2) über ihre bestandene Prüfung. Dabei entspricht die orthographische Form des Partizips „Böstanden!“ offensichtlich $A$ 's Normvorstellungen nicht, sodass er dieses in \#3 korrigiert. Bei der Gestaltung der Fremdkorrektur in \#3 nutzt $A$ die partielle Majuskelschreibung, um den Kontrast zwischen dem von ihm gelieferten Reparans „bEstanden“ und der Problemquelle aufzubauen. Die von $A$ vorgenommene Korrektur ähnelt weitgehend der Reparaturinitiierung in (192). Im vorliegenden Fall handelt es sich jedoch nicht mehr um eine Deklarativsatzfrage, sondern um einen Deklara- 
tivsatz, sodass die gelieferte Korrektur so gut wie keinen ,Angebotscharakter ‘ aufweist. Die Korrektur wird von $B$ explizit abgelehnt und der Gebrauch der Form böstanden (\#4) wird rechtfertigt, womit $B$ der impliziten Unterstellung von $A$, sie sei keine kompetente Schreiberin des Deutschen, entgegenwirkt (vgl. face work im Sinne von Goffman 1967).

Während bei den bisher in 6.2.1.6 diskutierten Beispielen Fremdinitiierungen analog zur gesprochenen Sprache funktionieren, wird bei dem in (194) vorliegenden candidate understanding auf eine lediglich in der schriftbasierten Kommunikation mögliche Ressource zurückgegriffen, nämlich auf ein Gleichheitszeichen (vgl. Mostovaia 2018: 73-75): ${ }^{140}$

(194) Auszug aus dem WhatsApp-Dialog \#4106:

\begin{tabular}{l} 
Schreiberin $A$ \\
$\qquad \begin{array}{c}\text { Alex sagt ungefähr jeden zweiten Tag er hätte kein Bock mehr aber ist } \\
\text { dann voll normal und keine Ahnung will Urlaub buchen und so voll } \\
\text { komisch }\end{array}$ \\
\hline Nachricht \#11 - 13.06.2016 - 19:23:05 \\
\hline Er meint ich sei knatschig \\
\hline Nachricht \#12 - 13.06.2016 - 19:23:05 \\
\hline Marianne ich bin auch gerade bei dem @- \\
\hline Nachricht \#13 - 13.06.2016 - 19:23:05 \\
\hline Oh \\
\hline Nachricht \#14 - 13.06.2016 - 19:24:05 \\
\hline Woher soll ich das wissen haha \\
\hline Nachricht \#15 - 13.06.2016 - 19:24:05 \\
\hline Nachricht \#16 - 13.06.2016 - 19:24:05 \\
\hline
\end{tabular}

140 Die folgende Analyse des Ausschnitts (194) wurde bereits in Mostovaia (2018) veröffentlicht. 


$\rightarrow$\begin{tabular}{|l|}
\hline Ja kein bock mehr $=$ Schlus machen $?$ \\
\hline Nachricht \#17 - 13.06.2016 - 19:25:05 \\
\hline
\end{tabular}

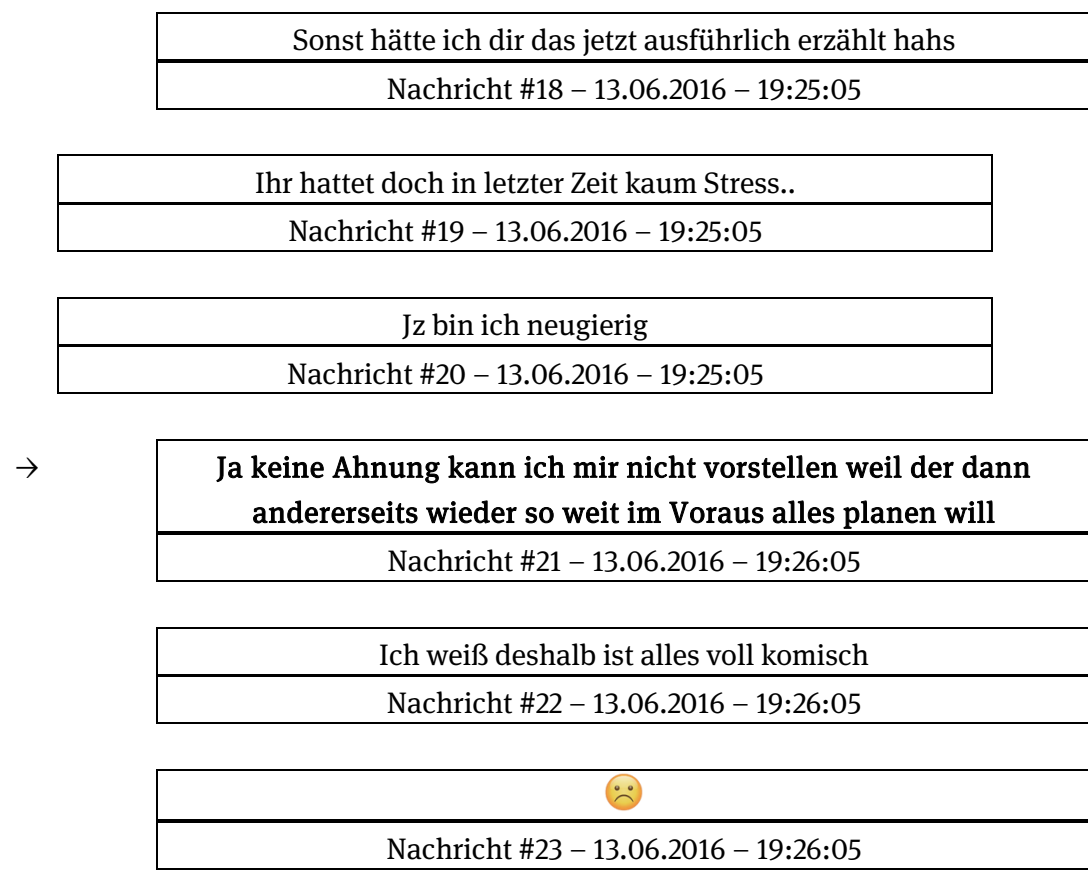

Am Anfang des Dialogs (194), der zwischen zwei Freundinnen verläuft, teilt Schreiberin $B$ mit, dass sie ein Beziehungsproblem mit ihrem Freund Alex hat und dass sie Schreiberin $A$ darüber beim Treffen am Freitag berichten will (nicht abgebildet). Nach einer Besprechung des bevorstehenden Treffens, das vom Freitag auf Samstag verlegt wird, kommt $B$ jedoch schon im Rahmen desselben WhatsApp-Dialogs auf ihr Beziehungsproblem zurück. Dabei beschwert sie sich bei $A$ darüber, dass ihr Freund sich komisch verhält, weil er immer öfter meint, dass er keine Lust auf eine Beziehung mit ihr hat. Anschließend liefert $B$ auch den Grund dafür, wieso sie die Besprechung ihres Beziehungsproblems ursprünglich auf Freitag verschieben wollte, nämlich, weil sie sich gerade bei ihrem Freund aufhält. In \#17 greift $A$ auf die von $B$ in \#11 geschilderte Situation zurück und thematisiert ihr Bedeutungsverstehensproblem mit dem Ausdruck „kein bock mehr“ (\#11). In diesem Zusammenhang liefert sie das candidate understanding „Schlus machen“, das sie mithilfe eines Gleichheitszeichens mit der von ihr wiederholten Problemquelle in Relation setzt. Dadurch werden potenzielle Schwierigkeiten bei der Erkennung der Problemquelle, die fünf Nachrichten zurückliegt, vermieden. Darauf reagiert $B$ in \#21, wobei sich das nach- 
richteninitiale „Ja“ nicht als eine Bestätigung in Bezug auf die Initiierungsnachricht, sondern entweder als Rückversicherungssignal oder als eine Bestätigung $A$ 's Annahme in \#19 interpretieren lässt. Zudem weist $B$ das von $A$ vorgelegte candidate understanding „Schluss machen“ implizit zurück. Anschließend zieht sie sogar den Wunsch ihres Freundes, alles im Voraus zu planen, als ein Argument hinzu, das ihrer Meinung nach für das Fortbestehen ihrer Beziehung sprechen soll.

Im Rahmen einer Fremdinitiierung in Form einer candidate solution kann mehr als ein Interpretationsvorschlag zur Ratifikation oder Korrektur angeboten werden. Das erfolgt mithilfe von Alternativfragen. Während sich im russischen Subkorpus lediglich ein einziger Beleg dafür findet, kommen in den deutschen Daten 13 Fälle vor:

(195) Auszug aus dem iMessage-Dialog \#3933:

Schreiberin $A$ Schreiber $B$

Dann muss ich mir was besonderes einfallen lassen :D Nene, dafür bin ich dann zu nett :D Oah, ich will Wochenende!

Nachricht \#30 - 26.01.2016 - 18:52:05

$\rightarrow$ ist aber gerade mal Mittwoch! Und ich will Urlaub :( Nachricht \#31 - 27.01.2016 - 11:15:05

$\rightarrow$ Oh Gott, nein :( Urlaub im Sinne von frei haben oder wegfahren? Nachricht \#32 - 27.01.2016 - 11:49:05

Im Sinne von wegfahren, natürlich! Kann das hier allmählich
alles nicht mehr sehen

Hab ich mir schon fast gedacht! Ich will das auch, einfach mal was anderes sehen wäre jetzt super! Wo willst du denn hin?

Nachricht \#34 - 27.01.2016 - 16:28:05

In (195) bietet Schreiber $B$ beispielweise zwei candidate understandings zu dem von ihm als ambig empfundenen Nomen Urlaub aus $A$ 's Beitrag (\#31) an. Die von $B$ in \#32 realisierte Fremdinitiierung nimmt die folgende Form an: die wiederholte Problemquelle + im Sinne von + candidate understanding 1 + oder + candidate understanding 2. Die der in Kap. 6.2.1.2 beschriebenen russischen Fremdinitiierung в смысле (,im Sinne von') parallele Konstruktion im Sinne 
von $+X$ wird in dem Fall sowohl bei der Präsentation der candidate understandings verwendet als auch in der Reparaturdurchführung mitwiederholt. Diese zwei Verwendungskontexten sind zwar für die mündliche Kommunikation im Russischen generell auch belegt (vgl. Goletiani 2003: 371-376), kommen allerdings in den analysierten russischen Kurznachrichten nicht vor.

Während in (195) die Problemquelle „Urlaub“ bei der Reparaturinitiierung in Form einer Alternativfrage (\#32) wiederaufgenommen und syntaktisch in die Initiierungsnachricht integriert wird, wird bei der Reparaturinitiierung in (196) auf das Zitieren der problematischen Nachricht zurückgegriffen, das Schreibern in der Messenger-Kommunikation zur Verfügung steht (vgl. auch Kap. 4.2):

(196) Auszug aus den für die russische Version der MoCoDa 2 erhobenen Daten (Zitieren einer WhatsApp-Nachricht in einer Fremdinitiierung):

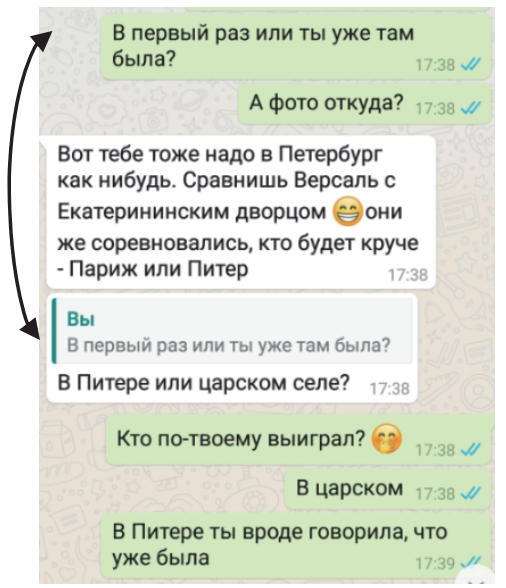

1 B: Bist du zum ersten Mal da oder warst du schon mal dort?

2 B: Und wo wurden die Fotos geschossen?

3 A: Du musst auch unbedingt mal Sankt Petersburg besuchen. Dann kannst du den Katharinenpalast und das Schloss Versailles miteinander vergleichen sie standen doch im Wettbewerb miteinander, welche Stadt ist cooler Paris oder Sankt Petersburg

4 A: Du

Bist du zum ersten Mal da oder warst du schon mal dort?

In Sankt Petersburg oder in Zarskoje Selo?

5 B: Wer hat deiner Meinung nach gewonnen?

\section{B: In Zarskoje Selo}

7 B: Du hast, glaube ich, bereits erzählt, dass du schon mal in Sankt Petersburg warst

Die in Form eines Zitats wiederholte problematische Nachricht enthält ein deiktisches Adverb „там“ (,da/dort'), das sich auf eine der beiden im vorherigen Verlauf des Dialogs genannten Städte bezieht, die von $A$ in \#4 als zwei candi- 
date references vorgelegt werden. Die zweite Referenz „[в] царском селе“ (\#4) wird von $B$ danach in \#6 bestätigt. Durch das Zitieren einer Nachricht, in der sich die Problemquelle befindet, wird ein Bezug zwischen dem Reparandum und der Reparaturinitiierung hergestellt, was v.a. in den Dialogen relevant ist, in denen die Nachricht mit der Problemquelle und diejenige mit der Reparaturinitiierung nicht unmittelbar aufeinander folgen. Die in (196) veranschaulichte Strategie kann im Kontext einer fremdinitiierten Selbstreparatur zudem auch angewandt werden, um eine Nachricht mit der Reparaturinitiierung in diejenige mit der Reparaturdurchführung zu integrieren. Somit wird also ebenfalls die eindeutige gegenseitige Zuordnung der beiden sequenziellen Bestandteile einer Reparatursequenz gewährleistet.

\subsubsection{Mehrere Fremdinitiierungen}

Ähnlich wie in der gesprochenen Sprache finden sich auch in der Kurznachrichtenkommunikation Fälle, bei denen mehr als eine Fremdinitiierung zum Anzeigen einer Problemquelle eingesetzt wird (vgl. doubles bei Egbert 2017). Dabei können unterschiedliche Initiierungsformate sowohl in derselben Nachricht liegen (vgl. (197) und (198)) als auch auf mehrere - i.d.R. zwei - Nachrichten aufgesplittet werden (vgl. (199), zum Splitting vgl. Beißwenger 2007: 245-253, 261-264 sowie Imo 2015a). Bei dem Vorhandensein zweier (seltener dreier) Fremdinitiierungen, die sich auf dasselbe Reparandum beziehen, bestätigt deren Reihenfolge weitgehend die These von Schegloff/Jefferson/Sacks (1977), dass jede weitere Fremdinitiierung, stärker ${ }^{\star}$ als die vorangehende ist. Anders formuliert: Die zweite Fremdinitiierung ist spezifischer als die erste, was sich darin niederschlägt, dass die zweite Reparaturinitiierung die Problemquelle genauer lokalisiert bzw. den Typ des vorliegenden Problems präziser anzeigt:

(197) Auszug aus dem SMS-Dialog \#2123:

\begin{tabular}{c} 
Schreiberin $A$ \\
$\rightarrow$\begin{tabular}{c|} 
Oh, Berlin ist schön! Und es gibt Duschen und feste Häuser. : ‘) Ich war \\
hier, nur für die Familie "weg". Wenig sprechen fällt mir übrigens \\
schwer :^), aber beim Lernenhält man ja eher den Mund. Ich baue schon \\
wieder komische Schreibweisen ein, muss wohl ins Bett. Gute Nacht!
\end{tabular} \\
\hline Nachricht \#8 - 04.04.2013 - 22:00 \\
\hline $\begin{array}{l}\text { Recht Du hast :) OK, verstehe. VORNAME allein zu Haus! ;) Ich verstehe } \\
\text { nicht: Welche komischen Schreibweisen? So oder so: Geruhsame Nacht! }\end{array}$ \\
\hline Nachricht \#9-04.04.2013 - 23:00 \\
\hline
\end{tabular}


Im vorherigen Verlauf des Dialogs (197) haben Schreiberin $A$ und Schreiber $B$ bereits mehrere Themen besprochen, die von ihrem Studium über ihre Freizeitaktivitäten bis zu ihren Vorstellungen über einen idealen Urlaub reichen. In \#8 leitet $A$ eine Verabschiedung ein, indem sie ihre nachlassende Aufmerksamkeit bzw. Schreibfähigkeit als Anlass zum Beenden des Dialogs nutzt und dabei ihre Schreibweise kritisiert („Ich baue schon wieder komische Schreibweisen ein, muss wohl ins Bett"). In \#9 arbeitet $B$ einzelne sprachliche Handlungen aus $A$ 's Nachricht der Reihe nach ab, indem er zuerst $A$ 's Meinung über Berlin unterstützt und danach ihre von ihm zuvor initiierte Reparaturdurchführung quittiert sowie einen weiteren Kommentar dazu abgibt. Erst danach kommt er zur Fremdinitiierung, die aus einem expliziten Teil „Ich verstehe nicht“, der die Art des Problems andeutet, sowie einer Teilwiederholung mit Fragewort „Welche komischen Schreibweisen?“, die die Problemquelle explizit benennt, besteht. In dem in der Datenbank abgebildeten Dialog fehlt jedoch die Reparaturdurchführung, sodass man nur darüber spekulieren kann, ob eine Problembearbeitung später erfolgt ist, da laut den vorliegenden Zeitangaben zwischen dem Verschicken von \#8 und \#9 bereits eine Stunde vergangen ist, sodass $A$ u.U. bereits im Bett war. Immerhin scheint der der Fremdinitiierung folgende Ausdruck „So oder so“ die konditionelle Relevanz der Reparaturdurchführung abzuschwächen.

Wie bereits erwähnt, können im Untersuchungskorpus bis zu drei Fremdinitiierungen miteinander kombiniert werden, was anhand von (193) aus dem russischen Teilkorpus illustriert werden kann:

(198) Auszug aus dem WhatsApp-Dialog \#4332:

Schreiber $A$

Schreiberin $B$

\begin{tabular}{|c|}
\hline Саш,ты жива? \\
\hline Sascha, lebst du noch? \\
\hline Nachricht \#1-24.11.2014-09:45 \\
\hline
\end{tabular}

$\rightarrow$

\begin{tabular}{|c|}
\hline Мне капут \\
\hline Ich bin erledigt \\
\hline Nachricht \#2 - 24.11.2014 - 09:50 \\
\hline
\end{tabular}

$\rightarrow$\begin{tabular}{|c|}
\hline ? работа? учёба? \\
\hline ? Job? Studium? \\
\hline Nachricht \#3 - 24.11.2014 - 09:50 \\
\hline
\end{tabular}


Если есть что-нибудь ,чем помочь могу..Я ещё дома торчу ,если тебе к 3 паре нужно что-то, скажи, найду-распечатаю

Wenn es etwas gibt, wobei ich dir helfen könnte.. Ich stecke noch zu Hause, wenn du etwas zur dritten Unterrichtsstunde brauchst, sag

Bescheid, ich kann das finden und ausdrucken Nachricht \#4 - 24.11.2014 - 09:52

\begin{tabular}{|c|}
\hline Да нужно \\
\hline Ja, ich brauche was \\
\hline Nachricht \#5 - 24.11.2014 - 10:23 \\
\hline
\end{tabular}

In der Fremdinitiierung in \#3 versucht $A$, den Grund für $B$ 's Probleme herauszufinden, die $B$ in $\# 2$ implizit angedeutet hat. Aus diesem Grund liefert er nach einem Fragezeichen, das analog $\mathrm{zu}$ unspezifischen Reparaturinitiierungen in der gesprochenen Sprache fungiert (vgl. Kap. 2.3.1.1 und 3.5.2.1), zwei candidate understandings. Daraufhin bietet $A$ seine Hilfe beim Beheben von $B$ 's Problemen an. $B$ nimmt $A$ 's Hilfsangebot an, liefert dabei allerdings keine explizite Reparaturdurchführung. Im weiteren Verlauf des Dialogs wird zumindest eines der beiden candidate understandings, und zwar „учёба“ (,Studium'), implizit bestätigt, da $A$ darum gebeten wird, nach Informationen zu einem bestimmten Thema für ein Geschichte-Seminar von $B$ zu suchen und diese auszudrucken.

Die in der WhatsApp-Kommunikation beobachtete Tendenz zum Aufsplitten von Nachrichten (vgl. Beißwenger 2007: 245-253, 261-264; Imo 2015a) zeigt sich ebenso bei einigen Fremdinitiierungen in den analysierten Daten, wie z.B. in (199), in dem sich zwei Studentinnen über ihr Schulpraktikum austauschen:

(199) Auszug aus dem WhatsApp-Dialog \#3356:

Schreiberin $A$ Schreiberin $B$

Там надо презентацию сделать? Или еще сдать в печатном варианте??

Sollten wie nur eine PowerPoint machen? Oder das Ganze noch in ausgedruckter Form abgeben?

Nachricht \#8 - 29.04.2014 - 14:19:05

$\rightarrow$

Презентация, я ее показывать классу буду

Eine PowerPoint-Präsentation, ich werde sie einer Klasse zeigen

Nachricht \#9-29.04.2014 - 14:21:05 


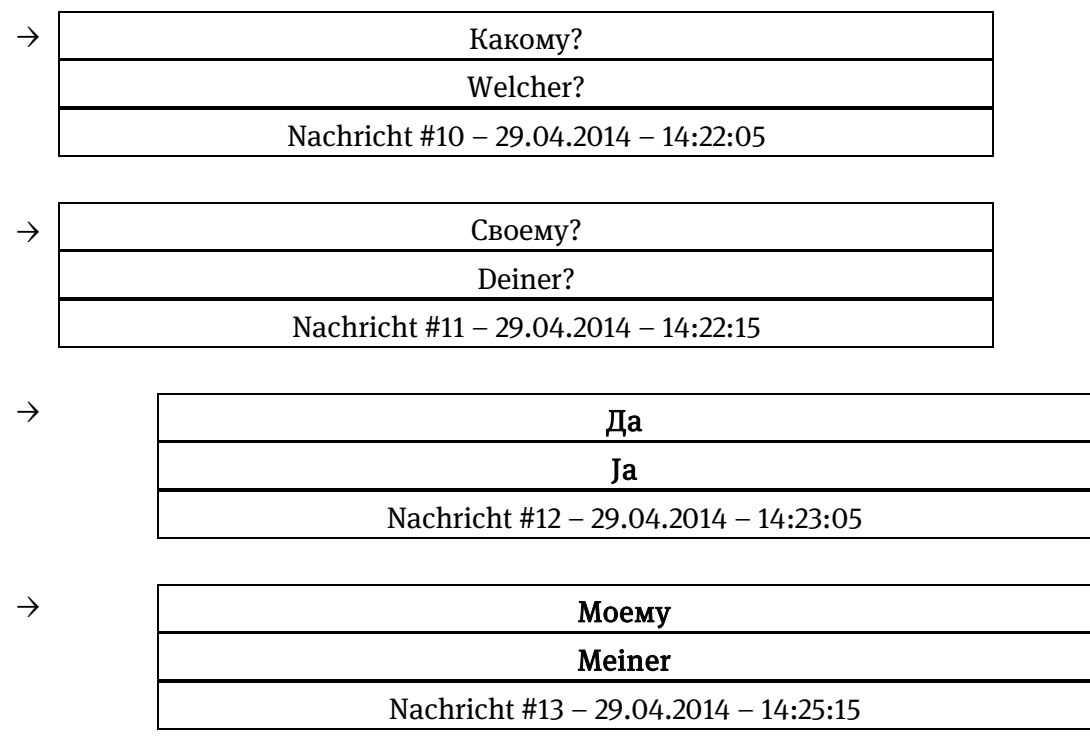

Im Laufe der Besprechung der für das Praktikum zu erbringenden Leistung klärt $B$ ihre Kommilitonin darüber auf, dass sie nur eine PowerPoint-Präsentation vorbereiten sollen, und fügt hinzu, dass sie diese in einer Unterrichtsstunde einer Klasse präsentieren wird. Schreiberin $A$ leitet danach eine Referenzreparatur an und benutzt dafür in \#10 das Format Fragewort („Какому?“ ,Welcher?`), wobei in der nächsten Nachricht eine zweite Fremdinitiierung in Form einer candidate reference „Своему?“ (,Deiner?‘, \#11) folgt. Diese candidate reference bestätigt $B$ in \#12 mit dem Responsiv „Да“ (,ja). Knapp zwei Minuten später liefert sie eine weitere Reparaturdurchführung, die man sowohl als eine Antwort auf \#10 als auch als eine weitere Bestätigung der Reparaturinitiierung in \#11 interpretieren kann, bei der das angebotene Possessivpronomen - zwar grammatisch angepasst - wiederholt wird.

\subsubsection{Reparanda}

\subsubsection{Rezeptionsprobleme}

Da akustische Verstehensprobleme ausschließlich in der medial mündlichen Kommunikation auftreten können, können diese entweder in einer MMSNachricht oder - im Falle der Messenger-Kommunikation - in einer Sprachnachricht verankert sein. Im gesamten Korpus findet sich lediglich ein einziger 
Fall (200), in dem das Reparandum in der WhatsApp-Sprachnachricht liegt, während die Bearbeitung des Problems in der medialen Schriftlichkeit erfolgt: ${ }^{141}$

(200) Auszug aus dem WhatsApp-Dialog \#4311:

Schreiberin $A$

Schreiberin $B$

\begin{tabular}{|c|}
\hline Wie gehts dirr?? Wir haben voll lane nicht geschrieben $2:$ : \\
\hline Nachricht \#1 - 24.10.2016 - 11:55:24 \\
\hline
\end{tabular}

\begin{tabular}{|c|}
\hline Jaaa das stimmt $\theta$ \\
\hline Nachricht \#2 $-24.10 .2016-12: 20: 16$ \\
\hline
\end{tabular}

\begin{tabular}{|c|}
\hline Und was machst du sooo heute??? \\
\hline Nachricht \#3-24.10.2016-12:20:25 \\
\hline
\end{tabular}

\begin{tabular}{|c|}
\hline Die uni hat angefangen heute Bin in münster \\
\hline Nachricht \#4 - 24.10.2016 - 12:26:58 \\
\hline
\end{tabular}

\begin{tabular}{|c|}
\hline Und du?? \\
\hline Nachricht \#5 - 24.10.2016 - 12:27:04 \\
\hline
\end{tabular}

$\rightarrow$

<Audio weggelassen>

Nachricht \#6 - 24.10.2016 - 12:27:06

Wiee cool ich freuu nichh für dichhh

Nachricht \#7 - 24.10.2016 - 12:31:40

Dann hast du ja jetzt auch ein ticket und kannst mich besuchen kommen

Nachricht \#8-24.10.2016-13:45:08

$\rightarrow$

Ich habe den namen des mädchens nicht gabz verstandne wen meinst $\mathrm{du}$ ?

Nachricht \#9 - 24.10.2016 - 13:45:31

$\rightarrow$

Welchen namen ?? $\Leftrightarrow$ das war dein name

Nachricht \#10 - 24.10.2016 - 13:46:08

141 Die Analyse von (200) wurde bereits in Mostovaia (2018) publiziert. 


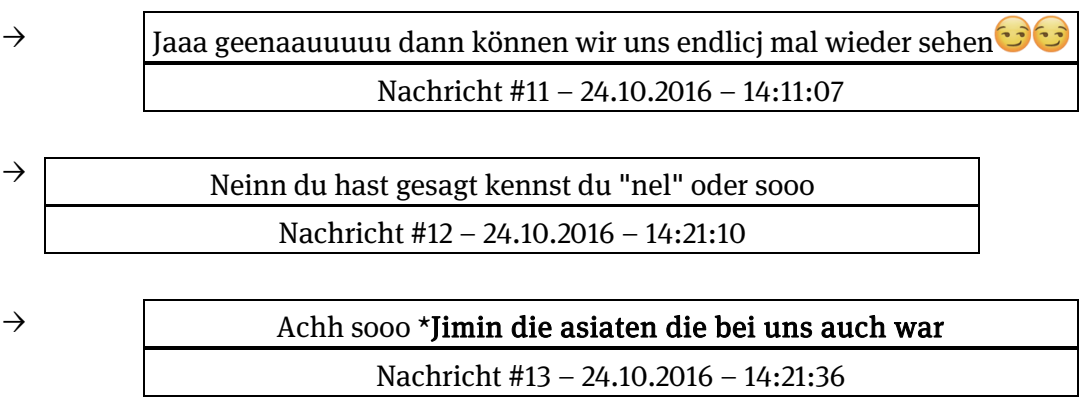

Nach einem kurzen Small-Talk zu Beginn der Dialogsequenz in (200) fragt Schreiberin $B$ ihre Kommunikationspartnerin nach ihren Plänen für den Tag. Nachdem Schreiberin $A$ diese Frage in Nachricht \#4 beantwortet hat, gibt sie diese zurück. Schreiberin $B$ verfasst ihre Antwort jedoch nicht in Form einer Textnachricht, sondern schickt eine Sprachnachricht (\#6), deren Inhalt leider bei der Einstellung des Dialogs in die MoCoDa nicht miterfasst wurde. Im späteren Verlauf des Dialogs stellt sich jedoch heraus, dass diese Sprachnachricht eine Problemquelle enthält. Aus den Nachrichten \#7-9 geht hervor, dass Schreiberin $B$ in ihrer Sprachnachricht anscheinend mehrere Themen angesprochen hat: Unter anderem hat sie dort einen Mädchennamen erwähnt, den Schreiberin $A$ jedoch akustisch nicht verstanden hat, worauf sie ihre Kommunikationspartnerin in Nachricht \#9 explizit hinweist. Damit initiiert sie die erste Reparatur, auf die Schreiberin $B$ in Nachricht \#10 eingeht. Dabei kann der erste Teil der Nachricht \#10, nämlich „Welchen namen ??“, der in Form einer Teilwiederholung mit Fragewort realisiert wird, als Reparaturinitiierung zu \#9 interpretiert werden (vgl. Schegloff/Jefferson/Sacks 1977: 368; Egbert 2009: 100). Im zweiten Teil wird mit „das war dein name“ die von $A$ initiierte Selbstreparatur durchgeführt, indem $B$ präzisiert, dass sie den Namen von Schreiberin $A$ selbst meinte. In der nächsten Nachricht weist Schreiberin $A$ jedoch diese Reparaturdurchführung zurück und versucht, den von ihr gemeinten Namen in Form eines candidate hearings ,"nel" “ zu liefern, welches sie in Anführungszeichen einschließt. Darauf reagiert Schreiberin $B$ mit dem Erkenntnisprozessmarker „Achh sooo“ (vgl. Golato/Betz 2008 sowie Imo 2009) und führt anschließend eine Selbstreparatur durch, indem sie den Mädchennamen „Jimin“ - mit einem vorangestellten Asterisk gekennzeichnet - verschickt und zusätzlich erläutert, woher sie bzw. die beiden Schreiberinnen diese Person kennen. Die Reparatur in (200) stellt dabei den einzigen Beleg in den untersuchten Daten dar, in dem ein Asterisk im Kontext einer fremdinitiierten und nicht selbstinitiierten Selbstreparatur gebraucht wird (vgl. Mostovaia 2018: 65-69). 
Während bei Problemen des Hörens ein akustisches Signal nicht dekodiert werden kann (vgl. Couper-Kuhlen/Selting 2018: 147), wird die verschickte Nachricht aus unterschiedlichen technischen Gründen nicht immer korrekt und/oder vollständig auf dem Gerät des Rezipienten dargestellt, sodass das Lesen verhindert wird. Somit könnte man Probleme in der Kurznachrichtenkommunikation, bei denen das akustische Signal oder der schriftlich repräsentierte Inhalt einer Nachricht nicht dekodiert werden können, unter dem Oberbegriff Rezeptionsprobleme fassen (es finden sich sechs Fälle von Rezeptionsproblemen in den deutschen und ein Fall in den russischen Daten). Ein Rezeptionsproblem kann zum Beispiel vorliegen, wenn nicht die komplette Nachricht, sondern nur ein Teil davon beim Kommunikationspartner ankommt, wie in Ausschnitt (201):

(201) Auszug aus dem SMS-Dialog \#1335:

Schreiberin $A$

Schreiberin $B$

\begin{tabular}{|c|}
\hline $\begin{array}{c}\text { Oh oh :-) ich hab grad NAME getroffen und natürlich lese ich nicht } \\
\text { meine studivz nachrichten :-D ha,hab ich mich gefreut! Ich hab mit } \\
\text { geredet und auf einmal hält sie mir den ring vor die nase :-) dann } \\
\text { sehen wir uns ja spätestens dann alle wieder.viel erfolg beim } \\
\text { schreiben heute. Meld dich mal,wenn d [Nachricht defekt] }\end{array}$ \\
\hline Nachricht \#1 - 01.06.2011 - 10:19 \\
\hline
\end{tabular}

\begin{tabular}{|c|}
\hline $\begin{array}{c}\text { Haha ich dachte natürlich du wüsstest es. Hab auch nicht } \\
\text { nachgeguckt, NAME2 hats gesagt. Mein handy zerstückelt immer } \\
\text { deine sms :( ich meld mich..̈̈h dann :) LG! }\end{array}$ \\
\hline Nachricht \#2 - 01.06.2011 - 10:31 \\
\hline
\end{tabular}

$\rightarrow$ Wenn du was weißt wegen deinem attest :-) nö,natürlich wusste ich nix...im studi bin ich nie mehr irgendwie. Bis dann! Liebste grüße Nachricht \#3 - 01.06.2011 - 10:54

Die erste Nachricht in (201), in der Schreiberin $A$ von ihrem Treffen mit einer gemeinsamen Freundin berichtet, die vor Kurzem ihre Verlobung per StudiVZNachricht bekannt gegeben hat, wird wegen ihrer Länge auf dem Gerät von Schreiberin $B$ nicht korrekt angezeigt, sodass das Ende der Nachricht fehlt. Stattdessen erscheint die Systemmeldung ,[Nachricht defekt]“ (\#1). Schreiberin $B$ reagiert in \#2 zunächst auf den für sie sichtbaren Teil der Nachricht und geht danach explizit auf das bei ihr aufgetretene technisch bedingte Problem ein, indem sie Schreiberin $A$ darauf hinweist, dass ihr Handy längere Nachrichten von $A$ immer zerstückelt. Außerdem fokussiert $B$ die Abbruchsstelle zusätzlich, 
indem sie auf den in \#1 abgebildeten Matrixsatz „Meld dich mal [...]“ mit „ich meld mich [...]“ (\#2) antwortet, geht aber mit dem abgebrochenen wennNebensatz, der außer der Subjunktion lediglich den Anfangsbuchstaben des nächsten Wortes $d$ beinhaltet, kreativ um. Auf den nicht abgebildeten Inhalt der Nachricht \#1 verweist $B$ mit dem Adverb dann, das zufällig oder absichtlich mit $d$ beginnt, genauso wie das erste Wort im Mittelfeld des abgebrochenen Nebensatzes aus \#1. Ihre Unsicherheit bezüglich des gelieferten, aber nicht ernst gemeinten candidate understandings bringt $B$ mithilfe des Hesitationsmarkers „äh“ sowie der zwei Auslassungspunkte, die wahrscheinlich eine Pause (und somit Nachdenken) symbolisieren sollten, zum Ausdruck. In der dritten Nachricht räumt Schreiberin $A$ der Reparatur den Vorrang ein und bearbeitet das vorliegende Rezeptionsproblem direkt am Anfang ihrer Nachricht, indem sie den kompletten wenn-Nebensatz erneut verschickt.

Während in (201) die Rezipientin der Problemquelle lediglich einen Teil der Nachricht nicht angezeigt bekommen hat, liegt in (202) ein Extremfall vor, bei dem die komplette Nachricht \#2 nicht lesbar ist:

(202) Auszug aus dem SMS-Dialog \#3413:

Schreiberin $A$

Schreiberin $B$

\begin{tabular}{|c|}
\hline Privet, ja v bonn, 1 ice na 15 min verspätung( \\
\hline Hallo, ich bin jetzt in Bonn. Der erste ICE hat 15 Minuten Verspätung( \\
\hline Nachricht \#1 - 05.12.2013 - 09:07:40 \\
\hline \\
\hline ??????, ??????. ???? ???, ??????? ?? ?????? ??????. \\
\hline ??????, ??????. ???? ???, ??????? ?? ?????? ??????. \\
\hline Nachricht \#2 - 05.12.2013 - 09:40:26 \\
\hline
\end{tabular}

$\rightarrow$

\begin{tabular}{|c|}
\hline Mne ot tebja odni voprosiki prishli \\
\hline Ich habe von dir nur lauter Fragezeichen bekommen \\
\hline Nachricht \#3 - 05.12.2013 - 09:41:31 \\
\hline
\end{tabular}

$\rightarrow$

Esli chto, poezgai na drugom poezde

Falls was, nimm einen anderen $\mathrm{Zug}$

Nachricht \#4 - 05.12.2013 - 09:50:59

\begin{tabular}{|c|}
\hline Horosho, poprobuju \\
\hline Ok, ich versuche es \\
\hline Nachricht \#5 - 05.12.2013 - 09:51:55 \\
\hline
\end{tabular}


Der in (202) vorliegende Auszug stammt aus dem russischen Subkorpus und ist in Translit verfasst (vgl. Kap. 4.1), und zwar aus dem Grund, dass das Handy von Schreiberin $A$ weder über die Spracheinstellungen für das Russische noch über die dazu benötigte physische Tastatur verfügt, während auf dem Handy von $B$ dagegen das Russische als Eingabesprache auf der virtuellen Tastatur eingestellt werden kann. In \#1 gibt $A$ ihrer Kommunikationspartnerin ihren aktuellen Standort durch und teilt außerdem mit, dass ihr Zug sich verspätet. $B$ 's Antwortnachricht \#2, die auf Russisch in kyrillischer Schrift verfasst wurde, konnte allerdings von $A$ 's Handy nicht richtig dekodiert werden, sodass auf dem Bildschirm ihres Handys eine Abfolge von Fragezeichen abgebildet wurde, die an einigen Stellen durch Leerzeichen oder Interpunktionszeichen voneinander getrennt sind. Darauf reagiert $A$ mit einer metakommunikativ expliziten Fremdinitiierung, in der sie $B$ auf das bei ihr entstandene technische Problem aufmerksam macht. Wie Nachricht \#4 zeigt, stellt sich Schreiberin $B$ darauf ein und wechselt ebenso ins Translit. Dabei kann man anhand der Zeichenzahl in Nachricht \#4 vermuten, dass nur ein Teil der problematischen Nachricht \#2 wiederholt wurde (vgl. Abb. 5).

\author{
Если что, поезжай на другом поезде. \\ "??????, ?????? ???? ???, ?????? ?? ?????? ??????."
}

Abb. 5: Hypothese zur Rekonstruktion der nicht dekodierten Nachricht \#2 aus (202)

Dies kann damit zusammenhängen, dass der erste Teil der Nachricht eine Gegenbegrüßung beinhaltet haben könnte (z.В. Привет, привет. (,Hallo, hallo.')) oder eine Begrüßung in Kombination mit einer Anrede (z.B. Привет, [Name]. (,Hallo [Name].')), da die Anrede im Russischen immer mit Kommata von dem Rest des Textes abgetrennt wird. Abgesehen davon hat die in \#4 dargestellte Reparaturdurchführung ihren interaktionalen Zweck erfüllt, was sich mit der von $A$ gelieferten Akzeptanz $B$ 's Ratschlags bestätigen lässt.

\title{
6.2.2.2 Referenzprobleme
}

Bei Referenzproblemen handelt es sich in erster Linie um diejenigen Fälle, bei denen ein in dem Kontext nicht ausreichend präziser Referenzausdruck vorliegt (vgl. (183) und (185); 24 Belege im russischen und 72 im deutschen Teilkorpus): 
(203) Auszug aus dem WhatsApp-Dialog \#3580:

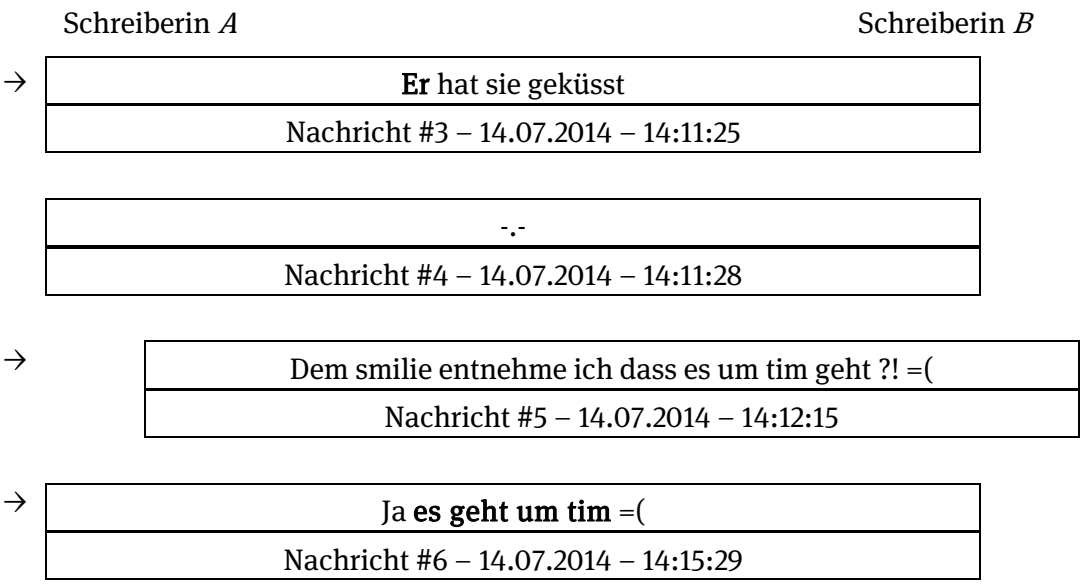

In \#3 teilt Schreiberin $A$ einer Freundin mit, dass jemand jemanden geküsst hat, ohne jedoch die Personen, auf die sie lediglich mit Personalpronomen „er“ und „sie“ referiert, genauer zu benennen. Schreiberin $B$ stellt in Nachricht \#5 eine Hypothese bezüglich der erwähnten männlichen Person auf, indem sie explizit darauf verweist, dass sie dabei das Emoticon „-.-“ aus Nachricht \#4 als Kontextualisierungshinweis nutzt und daher den Vornamen Tim als candidate reference vorlegt. Wie der hier nicht abgebildete Titel des Dialogs „küsse nie den Ex“ vermuten lässt, handelt es sich bei Tim höchstwahrscheinlich um $A$ 's Ex-Freund, sodass $B$ 's Schlussfolgerung, die in \#6 von $A$ bestätigt wird, nachvollziehbar wird.

Darüber hinaus finden sich in den untersuchten Kurznachrichtendialogen einzelne Problemquellen, bei denen die Referenz infolge eines Autokorrektureingriffs unkenntlich gemacht wurde, wie in dem WhatsApp-Verlauf (204), der zwischen zwei Freundinnen entstanden ist:

(204) Auszug aus dem WhatsApp-Dialog \#2918:

Schreiberin $A$ Schreiberin $B$

\begin{tabular}{|c|c|}
\hline \multirow[t]{3}{*}{$\rightarrow$} & Ты включая пойдешь сегодня?! \\
\hline & Gehst du heute einschaltend hin?! \\
\hline & Nachricht \#6 - 06.07.2013 - 13:42:50 \\
\hline
\end{tabular}

$\rightarrow \mid$\begin{tabular}{c|}
\hline Куда? \\
\hline Wohin? \\
\hline Nachricht \#7 $-06.07 .2013-14: 02: 00$ \\
\hline
\end{tabular}




\begin{tabular}{c}
$\rightarrow \quad$ В клюкву \\
\hline In „Kliukva“142 \\
\hline Nachricht \#8 - 06.07.2013 - 14:03:00 \\
\hline Незнаю даже, ты туда хочешь \\
\hline Bin mir nicht sicher, möchtest du hin? \\
\hline Nachricht \#9 $-06.07 .2013-14: 05: 00$ \\
\hline
\end{tabular}

Die von $B$ in \#6 gestellte Frage danach, ob Schreiberin $A$ irgendwohin hingeht, enthält zwar keine Ortsreferenz, die durch die Valenz des Verbes „пойдешь“ (,hingehen-2P.SG) gefordert wird, dafür aber das in dem Kontext unpassende Wort „включая“ ((a) ,einschaltend“; (b) ,einschließlich‘), das entweder als Adverbialpartizip (деепричастие) oder als Präposition fungieren kann. Eine Ortsreferenz wird in \#7 durch eine Fremdinitiierung in Form eines spezifischen Fragewortes, nämlich eines Interrogativadverbs „Куда?“ (,Wohin?`), eingefordert und in \#8 von $B$ auch geliefert. Dabei trägt die graphische Ähnlichkeit zwischen „включая“ (,einschaltend“/,einschließlich‘) und „В клюкву“ (,in Kliukva“, wo Kliukva der Name einer Bar ist) dazu bei, dass „включая“ als Problemquelle erkannt werden kann, die im Rahmen einer Substitution bearbeitet wird.

\subsubsection{Bedeutungsverstehensprobleme}

In der Kurznachrichtenkommunikation werden Bedeutungsverstehensprobleme ähnlich wie in der gesprochenen Sprache u.a. durch die Verwendung von ambigen oder vagen Lexemen (vgl. (195) und (205)) oder von Lexemen, deren Bedeutung dem Rezipienten nicht bekannt ist (vgl. (184) und (206)), ausgelöst (31 Fälle im russischen und 99 im deutschen Teilkorpus):

(205) Auszug aus dem SMS-Dialog \#2186:

Schreiberin $A$

Schreiberin $B$

Hey, also kurze Umfrage: was passt dir besser, 15. oder 21.? Lg Nachricht \#5 - 06.09.2012 - 21:11:00

$\rightarrow$

Also am 15. können jedenfalls VORNAME und ich. 21. ist bei mir eher schlecht... LG

Nachricht \#6 - 06.09.2012 - 21:13:00

142 „Kliukva“ ist der Name einer Bar. 


\begin{tabular}{|c|}
\hline $\begin{array}{c}\text { Heißt eher schlecht dass du gar nicht kannst? Oder müsstest du nur } \\
\text { machen dass du kannst? }\end{array}$ \\
\hline Nachricht \#7 - 06.09.2012 - 21:16:00
\end{tabular}

\begin{tabular}{|c|}
\hline $\begin{array}{c}\text { Ich bin von der Uni aus bis Freitag in STADT und weiß nicht } \\
\text { ob ich's schaffen würde ... : }\end{array}$ \\
\hline Nachricht \#8 - 06.09.2012 - 21:17:00 \\
\hline
\end{tabular}

Schreiberin $B$ plant ihre Geburtstagsfeier und sucht daher nach einem passenden Termin, indem sie eine kleine Umfrage unter ihren Gästen veranstaltet. In diesem Zusammenhang bietet $B$ ihrer Freundin $A$ in \#5 zwei Termine zur Auswahl, von denen $A$ für den ersten Termin plädiert (,am 15.“), während sie ihre Verfügbarkeit am zweiten der beiden möglichen Termine als „eher schlecht“ beschreibt. Schreiberin $B$ hakt bei der interpretationsbedürftigen Formulierung „eher schlecht“ nach und legt in \#7 zwei candidate understandings vor. Die von $A$ in \#8 gelieferte Reparaturdurchführung bestätigt keines der von $B$ angebotenen candidate understandings, sondern knüpft direkt an die Problemquelle an und gibt den Grund für das vage formulierte Reparandum an. Letztendlich beschließt Schreiberin $B$, ihren Geburtstag an den beiden Tagen zu feiern, da keiner der von ihr vorgeschlagenen Termine allen ihren Gästen gepasst hat.

Ein weiterer Auslöser für Bedeutungsverstehensprobleme liegt an der gelegentlichen Verwendung sprachlicher Ausdrücke, deren Bedeutungen dem Rezipienten nicht bekannt sind:

(206) Auszug aus dem SMS-Dialog \#2023:

Schreiberin $A$

Schreiberin $B$

\begin{tabular}{|c|c|}
\hline \multirow[t]{2}{*}{$\rightarrow$} & Vor der ORT kriegt man kostenlos lumumba und Glühwein :D \\
\hline & Nachricht \#8 - 11.12.2012 - 11:57 \\
\hline \multirow[t]{2}{*}{$\rightarrow$} & Was ist Lumumba?? Glühwein mag ich sowieso nicht :D \\
\hline & Nachricht \#9 - 11.12.2012 - 12:13 \\
\hline & Kakao mit rum :D \\
\hline & Nachricht \#10 - 11.12.2012 - 12:14 \\
\hline & Ja gut, mag ich dann auch nicht :D \\
\hline & Nachricht \#11 - 11.12.2012 - 12:15 \\
\hline
\end{tabular}


Schreiberin $A$ berichtet in \#8 über die Ausgabe kostenloser Getränke (,lumumba und Glühwein“) an einem Ort, an dem sie sich höchstwahrscheinlich zurzeit befindet. Die Bezeichnung des ersten Getränks „lumumba“ führt bei $B$ jedoch zu einem Bedeutungsverstehensproblem, das sie mit dem copular interrogative clause „Was ist Lumumba??“ (\#9) zum Ausdruck bringt. In \#10 liefert $A$ eine Umschreibung „Kakao mit rum“, deren Erhalt $B$ durch ihre Bewertung dieses Getränk quittiert. Das in \#9 sowie in \#10 und \#11 verwendete Emoticon „:D“ scheint in diesem Auszug lediglich eine phatische Funktion auszuüben (vgl. Imo 2015b: 146-148; auch Rahmung bei Pappert 2017: 189-191).

Ein weiterer Grund für Bedeutungsverstehensprobleme lässt sich auf die Besonderheiten der computervermittelten Kommunikation - u.a. auch der Kurznachrichtenkommunikation - zurückführen, nämlich auf die Besonderheiten der sequenziellen Organisation dieser Kommunikation (vgl. Beißwenger 2007 sowie Imo 2015a). Das Zusammenwirken mehrerer Faktoren, die die Produktion der Nachrichten beeinflussen, wie z.B. die Tippgeschwindigkeit, die Tendenz zum Aufsplitten von Nachrichten etc., führt dazu, dass in manchen Fällen die richtige Zuordnung der Antwortnachricht und somit die richtige Interpretation misslingen (vgl. auch das sogenannte Mühlenprinzip im Sinne von Wichter 1991):

(207) WhatsApp-Dialog \#4339:

Schreiber $A$ Schreiberin $B$

\begin{tabular}{|c|}
\hline Ты на парах? \\
\hline Bist du bei einer Lehrveranstaltung? \\
\hline Nachricht \#1 -29.10.2014 - 11:19 \\
\hline
\end{tabular}

\begin{tabular}{|c|}
\hline Куда мне подарок [имя] положить? или потом отдать? \\
\hline $\begin{array}{c}\text { Wo soll ich das Geschenk für [Name] hinlegen? Oder soll ich es dir später } \\
\text { übergeben? }\end{array}$ \\
\hline Nachricht \#2 - 29.10.2014 - 11:20 \\
\hline
\end{tabular}

$\rightarrow$

\begin{tabular}{|c|}
\hline Да \\
\hline Ja \\
\hline Nachricht \#3 $-29.10 .2014-11: 20$ \\
\hline
\end{tabular}

$\rightarrow$

\begin{tabular}{|c|}
\hline Что да? \\
\hline Was ja? \\
\hline Nachricht \#4 - 29.10.2014 - 11:21 \\
\hline
\end{tabular}




\begin{tabular}{|c|}
\hline Оставь на столе,где учебники \\
\hline Lass es auf dem Tisch, wo die Lehrbücher liegen \\
\hline Nachricht \#5 - 29.10.2014 - 11:22 \\
\hline
\end{tabular}

\begin{tabular}{|c|}
\hline В верхний ящик положил \\
\hline Ich habe es in die obere Schublade gelegt \\
\hline Nachricht \#6 - 29.10.2014-11:23 \\
\hline
\end{tabular}

Die ersten zwei Nachrichten aus (207) enthalten insgesamt drei Fragen, wobei in Nachricht \#2 außerdem eine Alternativfrage mit zwei einander ausschließenden Optionen gestellt wird. Darauf reagiert $B$ jedoch lediglich mit dem Responsiv „Да“ (,ja'), dessen Zuordnung von $A$ in \#4 als problematisch thematisiert wird. Dabei setzt sich die Fremdinitiierung aus dem Fragepronomen „Что“(,was') und dem wiederholten Responsiv „да“ (,ja‘) zusammen und stellt im Prinzip eine Ellipse für Что значит да? (,Was bedeutet $j a$ ?') dar. In der darauffolgenden Reparaturdurchführung knüpft $B$ an eine der in \#2 angesprochenen Varianten an und bittet $A$, das Geschenk auf den Tisch zu legen. Für (207) wäre aber auch eine andere Interpretation denkbar, und zwar, dass $B$ auf Nachrichten \#1 und \#2 jeweils mit einer separaten Nachricht geantwortet hat, sodass \#3 als eine bestätigende Antwort auf \#1 und \#5 als eine Reaktion auf \#2 gedacht waren, die durch die Fremdinitiierung in \#4 voneinander getrennt wurden. In diesem Fall fällt die in \#5 abgebildete Antwort auf die Frage in \#3 nur zufällig mit der zu \#4 passenden Reparaturdurchführung zusammen.

Des Weiteren können auch Emoticons für das Entstehen eines Bedeutungsverstehensproblems sorgen. Ein solcher Fall wurde bereits in (189) beschrieben, ein weiterer liegt in (208) vor:

(208) SMS-Dialog \#2120:

Schreiberin $A$

Schreiber $B$

Hey [Kosename]. Mein zug kommt gleich :-) Ich freue mich auf dich [animiertes Emoji] Ich liebe dich ganz feste. [animiertes Emoji]

Nachricht \#1 - 01.05.2013 - 12:47

Ok. Nimmst du den zug um 12.55 uhr? Ich liebe dich auch

Nachricht \#2 - 01.05.2013 - 12:49

\begin{tabular}{|c|}
\hline Genau :-) \\
\hline Nachricht \#3-01.05.2013-12:49 \\
\hline
\end{tabular}




\begin{tabular}{|c|}
\hline ok super. \\
\hline Nachricht \#4 - 01.05.2013 - 12:49 \\
\hline
\end{tabular}

\begin{tabular}{|c|}
\hline Und,ist der zug pünktlich? \\
\hline Nachricht \#5 - 01.05.2013 - 12:57 \\
\hline
\end{tabular}

\begin{tabular}{|c|c|}
\hline & :-) \\
\hline & Nachricht \#6 - 01.05.2013 - 12:58 \\
\hline & Das heißt wohl "ja"? \\
\hline & Nachricht \#7 - 01.05.2013 - 12:58 \\
\hline & Da hast du recht [animiertes Emoji] \\
\hline & Nachricht \#8-01.05.2013-12:59 \\
\hline
\end{tabular}

Schreiber $B$ ist auf dem Weg nach Hause zu seiner Frau, Schreiberin $A$, die er per SMS über seine Fahrt am Laufenden hält. Im Gegensatz zum Emoticon in (189) liefern die Zeitangaben sowie die inhaltliche Analyse der Nachrichten \#4 und \#5 aus (208) eine Evidenz dafür, dass das alleinstehende Emoticon „:-)“ in \#6 intendiert eingesetzt wird, und zwar als Antwort auf die Frage bezüglich der Pünktlichkeit des Zuges. Dieses Emoticon wird aufgrund seiner ,positiven Grundbedeutung، von $A$ als eine positive Antwort interpretiert, welche dem Kommunikationspartner als candidate understanding in \#7 zur Bestätigung vorgelegt wird, was in \#8 von $B$ auch getan wird.

Schließlich sollen nun die technischen Rahmenbedingungen der Kurznachrichtenkommunikation angesprochen werden, die im Untersuchungskorpus ebenso für Bedeutungsverstehensprobleme sorgen. Zum einen führt genauso wie bei Referenzproblemen die aktivierte Autokorrekturfunktion gelegentlich zu einem Bedeutungsverstehensproblem. Es finden sich allerdings insgesamt relativ wenige Beispiele, in denen ein durch die Autokorrektur oder durch einen Vertipper nicht mehr erkennbarer bzw. nicht im Kontext interpretierbarer sprachlicher Ausdruck problematisiert wird. In den meisten Fällen handelt es sich dabei um ein einziges Lexem, das betroffen ist (vgl. (210)), allerdings findet sich im Korpus auch ein Beispiel (209), in dem der unerwünschte Eingriff der Autokorrektur das gegenseitige Verstehen nicht nur punktuell gefährdet, sondern sich in mehreren aufeinander folgenden Nachrichten auswirkt und somit die gesamte Kommunikation erheblich beeinträchtigt. Diese Aneinanderreihung von Problemquellen kommt in (209) dadurch zustande, dass die Autokorrekturfunktion von Schreiberin $A$ auf die englische Sprache eingestellt ist, sodass die 
von ihr eingetippten deutschen Wörter teilweise in englische umgewandelt werden und die verschickten Nachrichten daher wenig Sinn ergeben:

(209) Auszug aus dem SMS-Dialog \#2117:

Schreiberin $A$

Schreiberin $B$

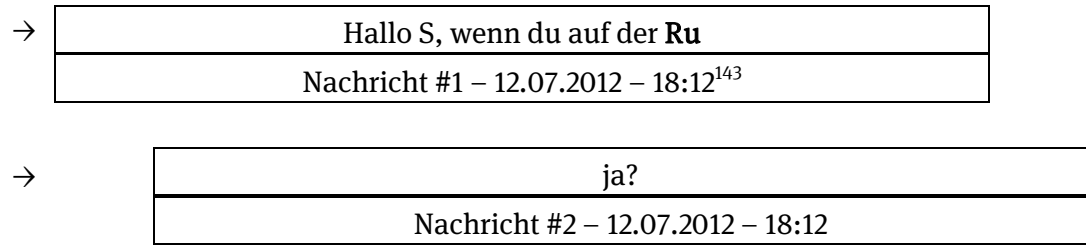

$\rightarrow$ Ruckfahrt langeweile hast, kannst du Mir mal men Schoenberg text fu

Nachricht \#3 - 12.07.2012 - 18:12

$\rightarrow$

\begin{tabular}{|c|}
\hline Das ding screwing vielleicht men sheiks \\
\hline Nachricht \#4 - 12.07.2012 - 18:12 \\
\hline
\end{tabular}

$\rightarrow$

\begin{tabular}{|c|}
\hline ich versteh kein Wort \\
\hline Nachricht \#5 - 12.07.2012-18:12 \\
\hline
\end{tabular}

$\rightarrow$

\begin{tabular}{|c|}
\hline Ich branches eminent text fur eminent beileidsbrief inenglisch \\
\hline Nachricht $\# 6-12.07 .2012-18: 12$ \\
\hline
\end{tabular}

$\rightarrow$

\begin{tabular}{|c|}
\hline boar Mama \\
\hline Nachricht \#7 $-12.07 .2012-18: 12$ \\
\hline
\end{tabular}

$\rightarrow$

\begin{tabular}{|c|}
\hline Have gestern beim googlen geese hen, dads spencer ende ma'am \\
\hline Nachricht $\# 8-12.07 .2012-18: 12$ \\
\hline
\end{tabular}

$\rightarrow$

\begin{tabular}{|c|}
\hline Marx gestorben it's \\
\hline Nachricht \#9 $-12.07 .2012-18: 12$ \\
\hline
\end{tabular}

$\rightarrow$ spencer ist gestorben? Nachricht \#10 - 12.07.2012 - 18:12

143 Nachrichten \#1-11 weisen die gleichen Zeitangaben auf, ab \#12 fehlen die Zeitangaben. 


\begin{tabular}{|c|c|}
\hline \multirow[t]{2}{*}{$\rightarrow$} & $\mathrm{Ja}$ \\
\hline & icht $\# 11$ - 12.07.2012 - 18:12 \\
\hline
\end{tabular}

\begin{tabular}{|c|}
\hline Mama les erstmal durch bevor du schreibst \\
\hline Nachricht \#12 - 12.07.2012 \\
\hline
\end{tabular}

\begin{tabular}{|c|}
\hline oh nein \\
\hline Nachricht \#13-12.07.2012 \\
\hline
\end{tabular}

\begin{tabular}{|c|}
\hline Der resetting \\
\hline Nachricht \#14 - 12.07.2012 \\
\hline
\end{tabular}

\begin{tabular}{|c|}
\hline stell mal auf deutsch um \\
\hline Nachricht \#15-12.07.2012 \\
\hline
\end{tabular}

Der ersetzt die Worte automatisch, da muss man jedes Wort dreimal schreiben, mal schnell ist nicht und der senden Knopf liegt so nah am loeschknopd, da schickt der schneller als der loscht Nachricht \#16 - 12.07.2012

Die erste Nachricht von $A$ wird unabgeschlossen abgeschickt, sodass das von $B$ gelieferte Rückmeldesignal ,ja?“ ihre Orientierung an die Fortsetzung der nicht kompletten Nachricht impliziert. In \#3 knüpft $A$ an das abgebrochene Wort „Ruckfahrt“ an und setzt ihre Nachricht fort. Wegen der Eingriffe der Autokorrektur ergeben sich für $B$ allerdings erhebliche Schwierigkeiten bei der Interpretation der Nachrichten \#3 und \#4. Diese signalisiert $B$ zwar explizit in \#5, das Problem setzt sich jedoch weiter fort, bis es Schreiberin $B$ in \#10 gelingt, zumindest die Nachrichten \#8 und \#9 zu entziffern und in \#10 ein candidate understanding für deren Inhalt anzubieten. Dieses wird von $A$ mit einem „Ja“ bestätigt. Danach folgt ein weiterer expliziter Hinweis auf das nicht gerade kooperative Schreibverhalten von $A$, gefolgt von einer Reaktion auf die Bestätigung des Todes der genannten Person. Erst nach dem Ratschlag, die Sprache bei der Autokorrektur zu wechseln, kann die Kommunikation erfolgreich fortgeführt werden.

Das Bedeutungsverstehensproblem in (210) ist ebenso wie in (209) durch das Auftreten eines technischen Problems bedingt. Dabei schickt Schreiber $A$ ihrer Tochter eine Nachricht per Sprachbefehl, d.h. er spricht auf sein Handy eine Nachricht. Diese wird jedoch nicht als Sprachnachricht verschickt, sondern wird von einer speziellen Spracherkennungssoftware bearbeitet und in Form 
einer medial schriftlichen Nachricht überführt, welche letztendlich an die Adressatin verschickt wird:

(210) Auszug aus dem Dialog \#1419:

Schreiber $A$

Schreiberin $B$

$\rightarrow$\begin{tabular}{c|}
$\begin{array}{c}\text { Hallo Sophie nutze jetzt mein neues Handy nummer siehst du ja liebe } \\
\text { grüße bis bald mal wieder googoo }\end{array}$ \\
\hline Nachricht \#1 - 16.10.2011 - 14:03:05 \\
\hline
\end{tabular}

\begin{tabular}{|c|c|}
\hline \multirow[t]{2}{*}{$\rightarrow$} & was heißt denn "googoo"? \\
\hline & Nachricht \#2 - 16.10.2011 - 14:05:05 \\
\hline & keine ahnung kommt beim sprechen ich bin dein vater \\
\hline & Nachricht \#3 - 16.10.2011 - 14:07:05 \\
\hline & Kanns mir bildlich vorstellen wie du ins Telefon brüllst :-) \\
\hline & Nachricht \#4 - 16.10.2011 - 14:10:05 \\
\hline
\end{tabular}

Bei der Spracherkennung läuft allerdings etwas schief, sodass am Ende der Nachricht \#1 das Wort „googoo“ erscheint, das im Laufe einer Fremdinitiierung in \#2 problematisiert wird. Dieses wird von $B$ wiederaufgenommen und in eine metasprachlich explizite Fremdinitiierung integriert, bei der das Verb heißen darauf hinweisen sollte, dass die Definition des wiederholten Elements benötigt wird, während die Modalpartikel denn dazu beiträgt, die erfragte Information als „bestimmungsbedürftig“ zu markieren und dem Schreiber $A$ die Verpflichtung zuzuschreiben, diese Problemquelle zu korrigieren (vgl. Deppermann 2009: 32). In \#3 liefert Schreiber $A$ das Reparans ,ich bin dein vater“ und erklärt außerdem explizit, wie das in \#1 aufgetretene Problem zustande kam.

\subsubsection{Erwartungsprobleme und Probleme der Akzeptabilität}

Erwartungsprobleme sowie Probleme der Akzeptabilität treten in denjenigen Fällen auf, bei denen die Problemquelle zu den Erwartungen, Erfahrungen, dem Wissensstand bzw. den Normvorstellungen des Rezipienten in Widerspruch steht (in den analysierten Daten ist das in 22 Belegen aus dem russischen und 52 aus dem deutschen Subkorpus der Fall). Da der jeweilige Kontext jedoch lediglich vereinzelt Informationen zu dem Wissensstand bzw. zu den Erwartungseinstellungen der Beteiligten liefert, lassen sich innerhalb dieser Gruppe der Reparanda kaum weitere Untergruppen bilden. Es kann höchstens zwischen Widersprüchen in Bezug auf allgemeines Wissen und denen in Bezug auf das 
,intern' geteilte Wissen (vgl. common ground in Clark/Marshall 1981; Clark 1996) unterschieden werden, wobei sich bei Fällen aus der zweiten Gruppe die Analyse aus der Beobachterperspektive als schwierig erweist.

Die erste Gruppe von Erwartungsproblemen, bei denen sich der entstandene Widerspruch auf allgemein bekannte Fakten bezieht, repräsentiert Ausschnitt (211):

(211) Auszug aus dem SMS-Dialog \#1423:

Schreiber $A$

Schreiberin $B$

\begin{tabular}{|c|}
\hline Maaaaaaaan!!die deutsche bahn ist fürn arsch.aber sowas von! \\
\hline Nachricht \#1 - 26.11.2011 - 07:34:05 \\
\hline
\end{tabular}

\begin{tabular}{|c|}
\hline ja kannst ja bald mit auto fahren \\
\hline Nachricht \#2 $-26.11 .2011-07: 36: 05$ \\
\hline
\end{tabular}

$\rightarrow$\begin{tabular}{|c|}
\hline $\begin{array}{c}\text { Aber bis dahin bin ich auf die penner angewiesen:(baustellen auf der } \\
\text { strecke u der schienenersatzverkehr ist einfach weg.ALTER! }\end{array}$ \\
\hline Nachricht \#3 - 26.11.2011 - 07:37:05 \\
\hline
\end{tabular}

\begin{tabular}{|c|}
\hline ??wie kann ein SCHIENENERSATZverkehr einfach weg sein??? \\
\hline Nachricht \#4-26.11.2011 - 07:40:05 \\
\hline
\end{tabular}

$\rightarrow \mid$\begin{tabular}{|c|}
\hline GUTE frage..er wird ja keine gäste befördert haben ne... \\
\hline Nachricht $\# 5-26.11 .2011-07: 44: 05$ \\
\hline
\end{tabular}

\begin{tabular}{|c|}
\hline wie dreist!!beschwer dich!!! \\
\hline Nachricht \#6 - 26.11.2011 - 07:58:05 \\
\hline
\end{tabular}

In \#1 beschwert sich Schreiber $A$ bei seiner Freundin über die Deutsche Bahn und teilt ihr später außerdem mit, dass er nicht mehr weiterkommt, da der Schienenersatzverkehr „weg“ (\#3) sei. In \#4 reagiert Schreiberin B auf A's Mitteilung bezüglich des Schienenersatzverkehrs mit einem iterierten Fragezeichen, gefolgt von einer weiteren Reparaturinitiierung in Form einer wie-Nachfrage, in deren Rahmen sie einen Teil von $A$ 's Beitrag wiederholt. Die iterierten Fragezeichen in der nachrichteninitialen und -finalen Position sowie die Majuskelschreibung des ersten Teils des Kompositums „SCHIENENERSATZverkehr“ implizieren $B$ 's Erwartungsproblem, da durch \#3 offensichtlich ein Widerspruch $\mathrm{zu}$ ihrem Wissen zu diesem Konzept bzw. zu ihren Erwartungen in Bezug darauf, wie dieses funktionieren soll, erzeugt wird. In \#5 zeigt $A$ mit der Floskel 
„GUTE frage“ an, dass er zwar B’s Wissen über den Schienenersatzverkehr teilt, die von ihm in \#3 skizzierte Situation jedoch tatsächlich diesem nicht entspricht, was $A$ mithilfe der anschließend gelieferten Erklärung zu beschreiben versucht.

Im Gegensatz dazu basiert das in (212) thematisierte Erwartungsproblem nicht auf der Diskrepanz zwischen den erhaltenen Informationen und dem Allgemeinwissen, sondern zwischen dem Inhalt von Nachricht \#3 und der Informiertheit von $B$ über Details aus $A$ 's Leben:

(212) Auszug aus dem SMS-Dialog \#3430:

Schreiberin $A$

Schreiberin $B$

\begin{tabular}{|c|}
\hline Privet, nu kak tam tvoe zdorovje? \\
\hline Hallo, wie fühlst du dich denn so? \\
\hline Nachricht \#1 - 28.04.2014-18:53:18 \\
\hline
\end{tabular}

Привет:-) Живу. Пью травяной отвар. Помогает. Ты как?

Hallo:-) Ich bin noch am Leben. Trinke einen Kräutertee. Das hilft. Wie geht's dir?

Nachricht \#2 - 28.04.2014 - 19:10:23

$\rightarrow$ Toze zivu. Poka ne piju, no mogu na4at $\Theta$ ti doma visdoravlivaesch?

Ich bin auch am Leben. Ich trinke noch nicht, kann aber bald anfangen $\hat{\theta}$ Kurierst du dich zu Hause aus?

Nachricht \#3 - 28.04.2014 - 19:12:30

$\rightarrow$

Да какой там. Приехала только с учебы. Зачем пить???

Und ob. Ich bin eben von der Uni zurückgekehrt. Wozu denn trinken???

Nachricht \#4 - 28.04.2014 - 19:15:51

$\rightarrow \quad$ Sa kompaniju $\hat{\theta}$ ti piescj, ja toze ho4u) a ja hotela tebja v kino posvat v konze nedeli, a ti es4e boleesch...

Aus der Solidarität $\hat{\theta}$ du trinkst, ich möchte auch) ich wollte dich ja fragen, ob du Ende der Woche ins Kino gehen wolltest, aber du bist noch krank...

Nachricht \#5 - 28.04.2014 - 19:18:16 


\begin{tabular}{|c|}
\hline Аааа.. мы можем в среду сходить. Часа в 3-4. \\
\hline Achsoo.. Wir können am Mittwoch hingehen. Gegen 15 oder 16 Uhr. \\
\hline Nachricht \#6 - 28.04.2014 - 19:19:15 \\
\hline
\end{tabular}

In (212) erkundigt sich Schreiberin $A$ nach $B$ 's Gesundheit, woraufhin sie in \#2 einen ausführlichen Bericht darüber erhält. In \#3 geht $A$ auf eine ebenso in \#2 von $B$ geäußerte Frage nach ihrem Befinden ein und stilisiert ihre Nachricht in einem witzigen Ton, indem sie die von $B$ in \#2 beschriebenen Handlungen wiederaufnimmt und auf sich selbst bezieht. Die scherzhafte Interaktionsmodalität des ersten Teils ihrer Nachricht markiert sie dabei mit dem Emoji $\hat{\theta}$ (vgl. Pappert 2017: 195-196). Das von $A$ verwendete Verb „piju“ (пью ,trinke‘) könnte man in dem Kontext sowohl analog zu \#2 im Sinne von ,Kräutertee trinken“ oder auch als ,Alkohol trinken“ interpretieren. Dabei würde man im ersten Fall auf eine einsetzende Erkrankung von $A$ schließen, während die zweite Interpretation Rückschlüsse darauf erlauben würde, dass $A$ evtl. einen Anlass hat, Alkohol zu trinken, von dem $B$ nichts weiß. Da $B$ offensichtlich die nicht ernsthaft gemeinte Aussage als ernsthaft aufgefasst hat, leitet sie in \#4 eine Fremdinitiierung ein, indem sie den Grund für das Trinken erfragt. A's Antwort zeigt, dass sie weder Gesundheitsbeschwerden noch sonstige Probleme hat, sondern das Ganze als Spaß gemeint hat.

Im gesamten Analysekorpus finden sich relativ wenige Reparaturen, die man eindeutig den Problemen der Akzeptabilität zuordnen kann (vgl. z.B. \#4204 in der MoCoDa). Dazu könnte aber u.a. die kreative Abschiedsfloskel „Kuss\&schuss“ (\#2) aus der Liebeskommunikation in (213) gezählt werden:

(213) SMS-Dialog \#1298:

Schreiberin $A$

Schreiber $B$

\begin{tabular}{|c|}
\hline Hey mein liebling, du fehlst mir soooooo!!!! \\
\hline Nachricht \#1 - 16.06.2011 - 00:09:03 \\
\hline
\end{tabular}

$\rightarrow$

huhu du mir auch!!!!!!ich liebe dich, Kuss\&schuss dein schatzi Nachricht \#2 - 16.06.2011 - 00:09:30

$\rightarrow \begin{gathered}\text { Oh mann, kuss und shuss?höhö, sagt man das unter computerspielern } \\ \text { so?du NERD!!!aber ein süßer...:) }\end{gathered}$

In \#3 zeigt Schreiberin $A$, dass die Floskel „Kuss\&schuss“, die Schreiber Bin \#2 verwendet, ihren Sprachvorstellungen nicht unbedingt entspricht und vermutet, dass es sich dabei um einen unter Computerspielern gebräuchlichen Aus- 
druck handelt. Der in der MoCoDa abgebildete Dialog endet allerdings mit Nachricht \#3, sodass offenbleibt, ob Schreiber $B$ später noch darauf reagiert hat oder nicht.

\subsubsection{Problematische sequenzielle Implikationen}

Bei problematischen sequenziellen Implikationen handelt es sich um Problemquellen, die durch das Auftreten einer aus der Sicht des Rezipienten sequenziell und/oder thematisch nicht passenden Äußerung ausgelöst werden. Weber (2014) hat für das Deutsche zwei Formate beschrieben, und zwar Reparaturinitiierungen mit und und wieso (vgl. Kap. 2.3.2.5 und 2.3.3.5). In den analysierten Daten lassen sich nur vereinzelt Fälle finden, die dieser Gruppe von Reparanda zugeordnet werden können. Es handelt sich hierbei um drei Beispiele im deutschen und zwei im russischen Korpus. Die Datenauswertung zeigte, dass sowohl in den deutschen als auch in den russischen Kurznachrichten ein Fragezeichen als Fremdinitiierung zum Anzeigen einer problematischen sequenziellen Implikation benutzt werden kann. Darüber hinaus tritt in einem russischen Dialog das Interrogativadverb почему (,wieso, warum') in der gleichen Funktion auf (vgl. (214)), während Schreiber in der deutschen Kurznachrichtenkommunikation auf das Fragepronomen was bzw. seine englische Variante what (vgl. (215)) zurückgreifen:

(214) Auszug aus dem Dialog \#2823:

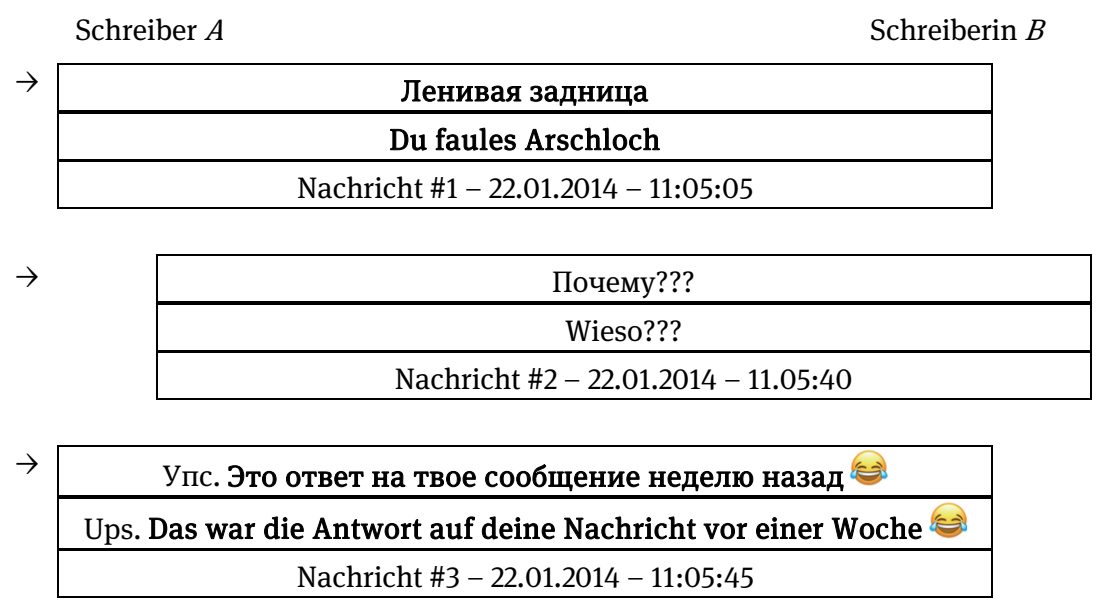

\begin{tabular}{|c|}
\hline Пфффф \\
\hline Pffff \\
\hline Nachricht \#4 -22.01.2014-11:06:05 \\
\hline
\end{tabular}




\begin{tabular}{|c|}
\hline Оно тогда не отправилось а сейчас я зашел и оно ушло \\
\hline $\begin{array}{c}\text { Sie wurde damals nicht zugestellt, und jetzt habe ich die App geöffnet } \\
\text { und sie wurde erneut versandt. }\end{array}$ \\
\hline Nachricht \#5 - 22.01.2014 - 11:06:05 \\
\hline
\end{tabular}

Die problematische sequenzielle Implikation besteht in (214) darin, dass Schreiber $A$ direkt in der ersten Nachricht eine negative, für $B$ gesichtsbedrohende Bewertung „Ленивая задница“ (,Du faules Arschloch') liefert, die von $B$ im Rahmen einer Reparaturinitiierung „Почему???“ (,Wieso???‘) hinterfragt wird. In \#3 reflektiert $A$ mit der Interjektion „Уп““ (,ups') sein Missgeschick und erläutert anschließend, dass er die in \#1 abgebildete Nachricht nicht vor ein paar Minuten, sondern vor einer Woche als Antwort auf eine Nachricht von $B$ verschickt hat. In seiner nächsten Nachricht setzt $A$ seine Erklärung fort und nimmt an, dass die Nachricht damals nicht abgeschickt wurde und er durch den Start der App das Verschicken der Nachricht unabsichtlich ausgelöst haben muss. Mit der lautmalerischen Interjektion „Пфффф“ (,pffff') drückt $B$ ihre Einstellung zu $A$ ’s Reparaturdurchführung bzw. Rechtfertigung der beleidigenden Bewertung aus.

In Ausschnitt (215) aus dem deutschen Teilkorpus, der ebenfalls eine problematische sequenzielle Implikation aufweist, bleibt die Nachricht mit der Problemquelle aus, wobei lediglich spekuliert werden kann, ob das Reparandum tatsächlich in einer zurückliegenden WhatsApp-Nachricht vorliegt oder ob dieses u.U. im Rahmen eines Face-to-Face-Gesprächs bzw. eines Telefonats realisiert wurde, das dem in (215) abgebildeten Dialog vorausging:

(215) WhatsApp-Dialog \#2451:

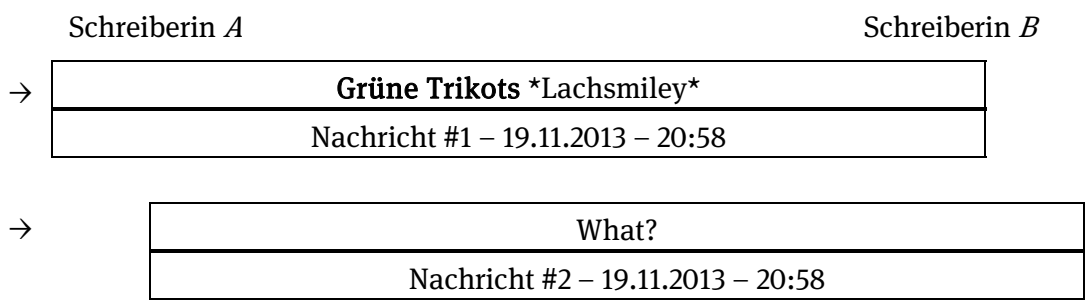

\begin{tabular}{|c|c|}
\hline & Fußball \\
\hline & Nachricht \#3 - 19.11.2013 - 20:59 \\
\hline
\end{tabular}

\begin{tabular}{|c|}
\hline Du hast doch gefragt welche Ausweich Finger die haben oder?! \\
\hline Nachricht \#4 - 19.11.2013 - 20:59 \\
\hline
\end{tabular}




\begin{tabular}{|c|}
\hline Jaa genau... wieder grün? \\
\hline Nachricht \#5 - 19.11.2013 - 21:00 \\
\hline
\end{tabular}

\begin{tabular}{|c|}
\hline Ja \\
\hline Nachricht \#6 - 19.11.2013 - 21:00 \\
\hline
\end{tabular}

Die in Nachricht \#1 gelieferte Mitteilung „Grüne Trikots“ erscheint Schreiberin $B$ sequenziell nicht eingebettet zu sein, daher leitet sie in \#2 eine Fremdinitiierung mithilfe eines englischen Interrogativs „What?“ ein, die von $A$ in zwei Schritten bearbeitet wird. Zunächst gibt $A$ ihrer Interaktionspartnerin in \#3 einen ersten Hinweis, indem sie die erwähnten Trikots mit einer bestimmten Sportart, nämlich mit Fußball, in Verbindung bringt. Danach beschreibt $A$ den Kontext, in dem die Farbe der Trikots von $B$ thematisiert wurde. Die Reaktion von $B$,Jaa genau...“ (\#5) zeigt, dass das Problem erfolgreich gelöst und die interaktionelle Relevanz der Nachricht \#1 von ihr nachvollzogen wurde.

\subsubsection{Reparaturdurchführungen}

Bezüglich der Reparaturdurchführung lassen sich in den analysierten Reparatursequenzen folgende Vorgehensweisen bzw. Strategien beobachten, die im Folgenden nach Häufigkeit des Auftretens im Korpus geordnet sind:

(i) Es erfolgt eine explizite oder eine implizite Reparaturdurchführung (65 Fälle im russischen und 184 im deutschen Teilkorpus).

(ii) Es liegt keine Reaktion auf eine geäußerte Fremdinitiierung vor (ausbleibende Reparaturdurchführung; sechs Fälle in den russischen und 24 in den deutschen Daten).

(iii) Schreiber reagieren zwar auf eine Fremdinitiierung, signalisieren aber, dass sie nicht im Stande sind, die adäquate Reparaturdurchführung zu produzieren (neun Fälle in den deutschen Kurznachrichtendialogen).

(iv) Schreiber weigern sich, eine Reparaturdurchführung zu liefern, und weisen die vorliegende Fremdinitiierung somit zurück (ein Fall im russischen und fünf Fälle im deutschen Subkorpus).

(v) Schreiber gehen zwar auf eine vorliegende Fremdinitiierung ein, die Problembearbeitung wird jedoch verschoben bzw. aus der vorliegenden Kommunikation ausgelagert und erfolgt dann beispielsweise bei einem späteren Face-to-Face-Gespräch (ein Fall in den russischen Daten). 


\subsubsection{Implizite und explizite Reparaturdurchführungen}

Bei zwei Fällen im deutschen und einem Fall im russischen Korpus könnte man von einer impliziten Reparaturdurchführung ausgehen. Dabei handelt es sich um Reparaturen, bei denen sich eine Problemquelle in einer Frage befindet, der eine Reparaturinitiierung in Form einer candidate solution folgt. Dabei wird diese Frage unter Vorbehalt der Tatsache beantwortet, dass die vorgelegte candidate solution zutrifft. Danach wird aber keine explizite Reparaturdurchführung unternommen, die eine Bestätigung oder eine Ablehnung des angebotenen Interpretationsvorschlages beinhaltet, sondern es wird der nächste erwartbare Schritt realisiert und somit die vorher gelieferte candidate solution implizit bestätigt (vgl. Deppermann/Schmitt 2008):

(216) Auszug aus dem WhatsApp-Dialog \#3032:

Schreiberin $A$

Schreiberin $B$

$\rightarrow$

[женское имя], ты видела сообщения в диалоге в контакте?

[Frauenname], hast du die Nachrichten in V kontakte ${ }^{144}$ gesehen?

Nachricht \#1 - 15.12.2013 - 15:32:15

$\rightarrow$

\begin{tabular}{|c|}
\hline Про вечеринку?Да \\
\hline Über die Party? Ja \\
\hline Nachricht \#2 - 15.12.2013 - 15:33:15 \\
\hline
\end{tabular}

$\rightarrow$

ты придешь? 3(

Gehst du hin? 3(

Nachricht \#3 - 15.12.2013 - 15:37:34

\begin{tabular}{|c|}
\hline К сожалению, пока что не знаю \\
\hline Leider weiß ich es noch nicht \\
\hline Nachricht \#4 - 15.12.2013 - 15:40:31 \\
\hline
\end{tabular}

Schreiberin $A$ macht ihre Freundin Schreiberin $B$ in (216) auf Nachrichten aufmerksam, die $B$ per Messenger-Funktion eines Sozialnetzwerks namens „B контакте“ (,V kontakte') erhalten haben muss. In \#2 liefert $B$ ein candidate understanding zur Problemquelle „сообщения“ (,Nachrichten'), indem sie deren thematische Zuordnung präzisiert („Про вечеринку?“,Über die Party?‘). Unter der Voraussetzung, dass ihr candidate understanding stimmt, liefert $B$ in

144 Vkontakte ist ein russisches Sozialnetzwerk. 
der gleichen Nachricht auch eine bestätigende Antwort auf $A$ 's Frage. Darauf reagiert $A$ mit einer Frage danach, ob $B$ dahingehen wird, womit sie dann $B$ 's candidate understanding implizit bestätigt, sodass keine explizite Reparaturdurchführung für die Verstehenssicherung notwendig ist.

In Bezug auf explizite Reparaturdurchführungen (64 Fälle in den russischen und 182 in den deutschen Daten) weisen die meisten im Korpus vorgefundenen Fälle die gleichen Bearbeitungsverfahren entsprechender Reparanda wie in der gesprochenen Sprache auf (vgl. Kap. 2.3.3). Bei Referenzproblemen wird die hinterfragte Referenz verdeutlicht und durch eine nicht-deiktische Form ersetzt (vgl. (183) und (185)). Im Falle von candidate references wird die vom Rezipienten angebotene Interpretation der problematischen Referenz entweder bestätigt oder abgelehnt und anschließend korrigiert. Analog dazu wird bei Bedeutungsverstehensproblemen vorgegangen, die mit candidate understandings aufgezeigt werden. Dabei erfolgt eine Bestätigung in beiden Fällen in den deutschen Daten überwiegend mithilfe der Antwortpartikel ja (u.a. mit deren Varianten jo, jep, jup). Damit ist die Verwendung des Responsivs да (,ja') vergleichbar, das in den russischen Daten für die Bestätigung von candidate solutions eingesetzt wird. Eine weitere Reparaturdurchführung, die ähnlich wie in der gesprochenen Sprache funktioniert, lässt sich bei candidate solutions beobachten, die in Form einer Alternativfrage realisiert werden. In solchen Fällen erfolgt die Bestätigung eines der beiden in der Fremdinitiierung angebotenen Elemente durch seine Wiederholung.

Lediglich in wenigen Einzelfällen weicht die Reparaturdurchführung davon ab, wobei das nicht unbedingt automatisch zum Scheitern einer Reparatursequenz führt. Als Beispiel dafür dient Auszug (217), in dem Schreiberin $A$ sich bei ihrer Schwester erkundigt, wann sie und die Mutter von den beiden in die Stadt, wo $A$ sich gerade befindet, ankommen:

(217) Auszug aus dem SMS-Dialog \#2685:

Schreiberin $A$

Schreiberin $B$

Ich hab gehört, du und Mama kommt ihr kommt morgen hier her.?

Ich freu mich. Kannst du mir aber kurz vorher bitte Bescheid geben wann ihr losfährt und wann ihr in STADT seid?

Nachricht \#1 - 03.01.2014 - 14:05:05

Hey! Ja richtig. VORNAME kommt auch mit. Wir wären so gegen

Nachmittag da.

Nachricht \#2 - 03.01.2014 - 14:09:05 


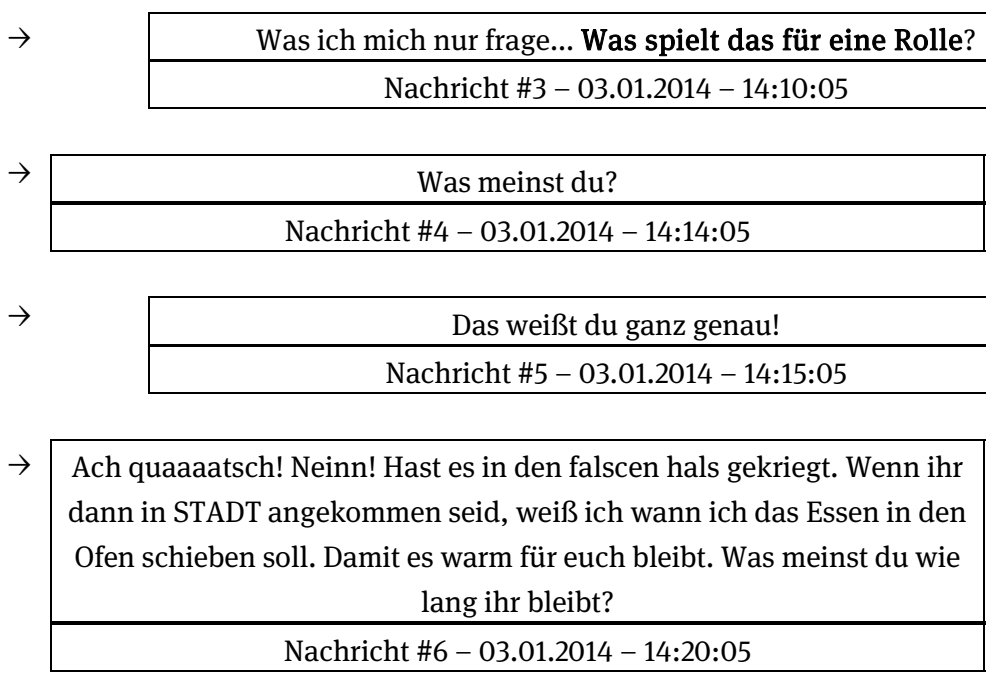

Schreiberin $B$ teilt in \#2 mit, dass sie zu dritt kommen, und reagiert anschließend auf die Nachfrage über deren Ankunftszeit. In \#3 unterstellt $B$ jedoch ihrer Schwester, kein ,Recht' auf die in \#1 gestellte Frage zu haben. Darauf reagiert $A$ mit einer metakommunikativ expliziten Fremdinitiierung „Was meinst du?“ (\#4). ${ }^{145}$ Anstatt ihre Äußerung in \#3 zu erklären, weist Schreiberin $B$ jedoch ihre Schwester in \#5 darauf hin, dass sie die Antwort auf ihre Frage selbst kennt. Diese Unterstellung scheint interaktional zu funktionieren, da $A$ den von $B$ angedeuteten Grund für die Frage nach der Ankunftszeit negiert und ihre Frage damit rechtfertigt, dass sie das Essen rechtzeitig aufwärmen wollte.

Ein weiteres Beispiel, in dem die Fremdinitiierung nicht mit einer dafür typischen Reparaturdurchführung eingelöst wird, liegt in Auszug (218) aus einem Dialog zwischen zwei Freunden vor:

(218) Auszug aus dem Dialog \#2252:

Schreiber $A$

Schreiber $B$

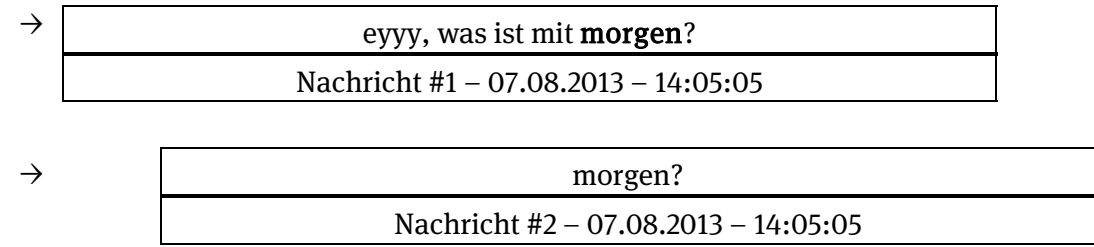

145 Zur Rolle der Metaebene in Streitgesprächen vgl. Watzlawick (1999: 39-50). 


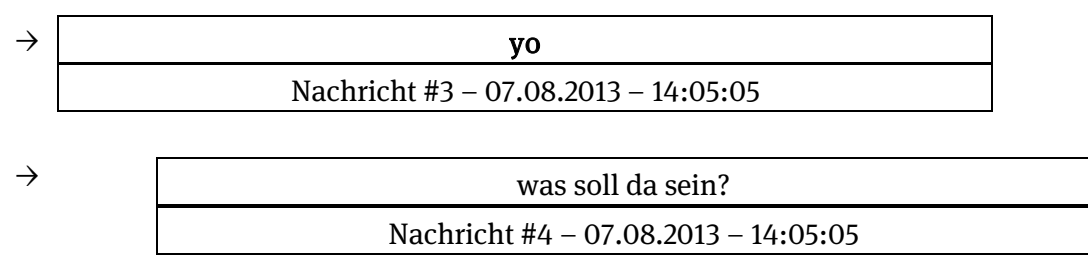

\begin{tabular}{|c|c|}
\hline & wir wollten doch nach ORT fahren \\
\hline & Nachricht \#5 - 07.08.2013 - 14:05:05 \\
\hline
\end{tabular}

scheiße, sorry kumpel. hab ich voll vergessen. bin mit WEIBLICHER NAME verabredet...

Nachricht \#6 - 07.08.2013 - 14:05:05

super. bist ja echt ein toller kumpel. kaum hast du ne perle...

Nachricht \#7 - 07.08.2013 - 14:05:05

Direkt am Anfang des Dialogs (218) fragt Schreiber $A$, was mit morgen sei. Darauf reagiert Schreiber $B$ mit einer Fremdinitiierung in Form der Teilwiederholung „morgen?“ (\#2), die als Initiierungsformat bei akustischen Problemen, Bedeutungsverstehensproblemen oder Erwartungsproblemen eingesetzt werden kann (vgl. Couper-Kuhlen/Selting 2018: 197-200; vgl. Kap. 2.3.1.4). Ein akustisches Problem bzw. - angepasst an die Kommunikationsbedingungen der medialen Schriftlichkeit - ein Rezeptionsproblem (vgl. Kap. 6.2.2.1) liegt in dem Fall nicht vor, weil der Inhalt von Nachricht \#1, die die Problemquelle enthält, richtig angezeigt wird. Ein Bedeutungsverstehensproblem lässt sich auch ausschließen, weil $B$ sich im weiteren Verlauf des Dialogs als erfahrener Sprecher bzw. Schreiber des Deutschen erweist und daher keine Probleme mit der Bedeutung von „morgen“ haben kann. Sogar ein für die vorliegende Fremdinitiierung eher untypisches Referenzproblem kann ausgeschlossen werden, da jede Nachricht mit einer Zeitangabe versehen ist und die beiden Schreiber sich im Gegensatz zu (163) in einer Zeitzone befinden. Der einzige Problemtyp, der also übrig bleibt, ist das Erwartungsproblem. Bei einem Erwartungsproblem würde man z.B. mit einer Erklärung, einer expliziten Auseinandersetzung mit dem entstandenen Widerspruch oder mit einer Veränderung des Wissensstandes des Kommunikationspartners rechnen (vgl. Couper-Kuhlen/Selting 2018: 200). Im vorliegenden Beispiel besteht die Reparaturdurchführung jedoch lediglich aus der Gesprächspartikel „yo“ (\#3), die man eher als Reparaturdurchführung bei einem Bedeutungsverstehens- oder Referenzproblem erwarten würde. Den Einsatz einer Reparaturdurchführung, die für eine bestimmte Kombination aus 
einem Reparandum und einer Reparaturinitiierung als nicht typisch erscheint, bezeichnen Couper-Kuhlen/Selting (2018: 142) als Rekategorisierung. Die in (218) vorliegende Rekategorisierung erfüllt in der analysierten Interaktion eine wichtige pragmatische Funktion: Die Wahl der nicht prototypischen Reparaturdurchführung in einem Kontext, in dem die Art des Problems (im Gegensatz z.B. $\mathrm{zu}$ (219)) eindeutig erkennbar ist, erzeugt eine Implikatur: Schreiber $B$ wird damit implizit vorgeworfen, dass seine Fremdinitiierung eigentlich nicht gerechtfertigt ist, da er bereits über die angefragten Informationen verfügen sollte. Die erneute, diesmal aber etwas präzisere, Fremdinitiierung in \#4 zeigt, dass $B$ dennoch nicht im Stande ist, zu dem Zeitpunkt die benötigten Informationen über den morgigen Tag in seinem Gedächtnis abzurufen. Daher erinnert $A$ im Laufe der Reparaturdurchführung in \#5 seinen Kommunikationspartner an ihre gemeinsamen Pläne. Diese Reparaturdurchführung führt im Gegensatz zur ersten zum Erfolg, da $B$ sich an die Abmachung erinnert, was er explizit in \#6 zeigt, und sich dafür entschuldigt, dass er bereits eine andere Verabredung an dem Tag hat, da er den Termin mit $A$ vergessen hat.

Die Analyse der Zusammenhänge zwischen einer Reparaturinitiierung und -durchführung wird vereinzelt dadurch erschwert, dass eine (meist unspezifische) Fremdinitiierung im gegebenen Kontext auf unterschiedliche Reparanda hinweisen könnte, sodass sich die Interpretation der Fremdinitiierung seitens des Rezipienten der Problemquelle nicht eindeutig rekonstruieren lässt:

(219) Auszug aus dem SMS-Dialog \#3563:

Schreiber $A$

Schreiberin $B$

Na gut, das ist ja schon mal etwas! Und wie gehts dir jetzt? <3 Ja total!! Vor allem hab ich nen richtig coolen ansatz, voll interessant, ich hoffe die erlaubt mir das! :))

Nachricht \#8 - 27.06.2014 - 12:10:05

Schön :) Ganz ok soweit..

Nachricht \#9-27.06.2014-12:11:05

Jaa kann ich dir gleich erzählen wenn du möchtest :) Was heißt ganz ok soweit? $<3$

Nachricht \#10 - 27.06.2014 - 12:12:05

\begin{tabular}{|c|c|}
\hline \multirow[t]{2}{*}{$\rightarrow$} & $\mathrm{Kla}$ \\
\hline & Nachricht \#11 - 27.06.2014 - 12:13:05 \\
\hline
\end{tabular}




$\rightarrow \quad \begin{gathered}\text { Hm okay... Na dann muss ich machen dass es dir gut geht! :-) Hast du } \\
\text { denn interesse dran? ;-) }\end{gathered} \mid$\begin{tabular}{c} 
Nachricht \#12 - 27.06.2014 - 12:19:05 \\
\hline
\end{tabular}

$\rightarrow$

\begin{tabular}{|c|}
\hline Hö? \\
\hline Nachricht \#13 $-27.06 .2014-12: 19: 05$ \\
\hline
\end{tabular}

$\rightarrow$

\begin{tabular}{|c|}
\hline Dass ich dir von meiner hausarbeit erzähle? ;) \\
\hline Nachricht \#14 - 27.06.2014 - 12:22:05 \\
\hline
\end{tabular}

\begin{tabular}{|c|}
\hline Ja klar wir können da mal drüber reden.. \\
\hline Nachricht \#15 - 27.06.2014 - 12:24:05 \\
\hline
\end{tabular}

Schreiberin $B$ fragt ihren Freund nach seinem Befinden und berichtet ihm über einen spannenden Ansatz, den sie in ihrer Hausarbeit anwendet und welchen sie zum ersten Mal in \#6 (nicht abgebildet) erwähnt hat. In \#9 gibt $A$ eine Bewertung über $B$ 's Begeisterung für ihre Hausarbeit ab und beschreibt sein Befinden als „Ganz ok soweit“. In der darauffolgenden Nachricht stimmt $B$ seiner Bewertung $\mathrm{zu}$, fragt nach, ob von seiner Seite aus Interesse an mehr Informationen über die Hausarbeit bestehe, und stuft den Ausdruck „ganz ok soweit“ als reparaturbedürftig ein. In \#11 arbeitet $A$ Schritt für Schritt einzelne kommunikative Handlungen aus der vorherigen Nachricht ab. Er zeigt seine Bereitschaft, detailliert über $B$ 's Hausarbeit informiert zu werden, und liefert die von $B$ eingeforderte Reparaturdurchführung „nicht gut aber ok“. Als Reaktion darauf gibt $B$ ihre Absicht bekannt, etwas zu tun, damit es $A$ besser geht, wobei sie diese Mitteilung mit einer modifizierten Form ihrer Anfrage in \#10 koppelt. Diese Kombination löst eine Fremdinitiierung in Form der Partikel „Hö?“ (\#13) aus. Dadurch, dass die Partikel hö zu den unspezifischen Initiierungsformaten zählt, lässt sich die somit zustande gekommene Konstellation aus Problemquelle und Reparaturinitiierung mindestens auf dreierlei Weise deuten. An dieser Stelle müssen nun die drei potenziellen Interpretationsmöglichkeiten kurz erläutert werden. Erstens kann der deiktische Bezug des Präpositionaladverbs „dran“ aus \#12 von $A$ anders als von $B$ intendiert verstanden werden. Dabei würde $A$,dran“ mit dem ersten Teil der Nachricht \#12 „Na dann muss ich machen dass es dir gut geht!" in Verbindung bringen, was u.U. zu einem Erwartungsproblem führen würde. Die zweite Option besteht darin, dass Schreiber $A$ die Frage „Hast du denn interesse dran? ;-)“ aus \#12 als modifizierte Wiederholung $B$ ’s Anfrage in \#10 deutet (,Jaa kann ich dir gleich erzählen wenn du möchtest :)“), die er eigentlich bereits in \#11 mit einer Bestätigung quittiert hat. In diesem Fall könnte 
die Notwendigkeit, im Grunde genommen dieselbe Frage erneut zu beantworten, ebenfalls ein Erwartungsproblem auslösen. Die dritte Möglichkeit hat damit zu tun, dass $A$ sich der Ambiguität des deiktischen Ausdrucks „dran“ im vorliegenden Kontext bewusst ist, sich aber nicht entscheiden kann, ob sich „dran“ auf das Erzählen über die Hausarbeit bezieht oder im Zusammenhang mit B'S Absicht steht, etwas gegen seine schlechte Laune zu unternehmen. Daher wäre es potenziell nicht auszuschließen, dass das hö in dem Fall auf ein Referenzproblem verweist. Abgesehen davon, dass hö in der Forschungsliteratur eigentlich nicht als prototypische Fremdinitiierung bei Referenzproblemen beschrieben wird, wird das in (219) vorliegende Reparandum von $B$ trotzdem als solches bearbeitet (vgl. \#14). Dabei lässt sich der dass-Nebensatz als nachgeliefertes Attribut zu „dran“ aus \#12 betrachten, wodurch dieses disambiguiert wird. Ob $A$ tatsächlich ein Referenzproblem hatte oder ob er sein Erwartungsproblem thematisieren wollte, was von $B$ anders gedeutet wurde, lässt sich leider dem weiteren Kontext nicht entnehmen. Es kann lediglich festgehalten werden, dass die in \#14 angebotene Reparaturdurchführung anscheinend ihren Zweck erfüllt und zur Verstehenssicherung beigetragen hat, da keine weitere Reparaturinitiierung unternommen wurde.

Der letzte Aspekt, der v.a. in Bezug auf explizite Reparaturdurchführungen noch erwähnt werden muss, hängt mit den sogenannten multiples im Sinne von Schegloff (2000) sowie Egbert (2017) zusammen. Dabei handelt es sich um zwei und mehr Fremdinitiierungen, die in unterschiedlichen Nachrichten des Rezipienten der Problemquelle realisiert werden, aber sich auf dasselbe Reparandum richten, da die vorangehende Reparaturinitiierung ihren Zweck nicht erfüllt hat (vgl. (218)). Multiples machen einen relativ geringen Anteil der analysierten Reparaturen aus. In den deutschen Daten finden sich zwölf multiples unter insgesamt 222 Reparaturen, während sich in den russischen Daten die Anzahl auf fünf von 73 Fällen beläuft. Die niedrige Frequenz von multiples zeugt davon, dass der Reparaturmechanismus in der medial schriftlichen Kommunikation ein effizientes Instrument für das Beheben verschiedener Probleme darstellt, sodass die meisten Reparanda bereits bei der ersten Reparaturdurchführung erfolgreich bearbeitet werden.

\subsubsection{Ausbleibende Reparaturdurchführungen}

$\mathrm{Zu}$ ausbleibenden Reparaturdurchführungen (sechs Fälle im russischen und 24 im deutschen Teilkorpus) wurden beispielsweise solche Fälle gezählt, bei denen die jeweilige Reparaturinitiierung die letzte Nachricht in dem für die Datenbank gespendeten Dialog darstellt, sodass es sich nicht feststellen lässt, ob die Reparaturdurchführung jeweils wirklich nicht geliefert wurde oder lediglich in der 
Datenbank nicht abgebildet ist (vgl. (197) und (213); acht Fälle in den deutschen Daten).

Zudem finden sich innerhalb dieser Kategorie Fälle, bei denen die Kommunikation nach einer Reparaturinitiierung zwar fortgesetzt, aber die Reparaturdurchführung trotzdem nicht realisiert wird. Dabei scheint das Ausbleiben der Reparaturdurchführung bei solchen Belegen v.a. darauf zurückzuführen zu sein, dass die Problembearbeitung sich nicht (mehr) als relevant für die bestehende Kommunikation erweist oder die Reparaturinitiierung übersehen bzw. zugunsten anderer wichtigerer kommunikativer Aufgaben ignoriert wurde.

Ein Beispiel für eine ausbleibende Reparaturdurchführung, deren Notwendigkeit sich bereits erübrigt hat, liefert der WhatsApp-Dialog (220):

(220) WhatsApp-Dialog \#3906:

Schreiberin $A$

Schreiberin $B$

\begin{tabular}{|c|}
\hline Hey! Kommst du gleich zu höfische Literatur? \\
\hline Nachricht \#1 - 26.10.2015 - 11:59:00 \\
\hline
\end{tabular}

\begin{tabular}{|c|}
\hline Hey Ja ich werde da sein! Treffen wir uns dort? \\
\hline Nachricht \#2 - 26.10.2015 - 12:01:00 \\
\hline
\end{tabular}

\begin{tabular}{|c|}
\hline $\begin{array}{c}\text { Jo! Falls du früher da bist, könntest du dann vielleicht 3plätze frei } \\
\text { halten? }\end{array}$ \\
\hline Nachricht \#3 - 26.10.2015 - 12:10:00 \\
\hline
\end{tabular}

\begin{tabular}{|c|}
\hline Jao. Äh bin aber erst so 5 nach da \\
\hline Nachricht \#4 - 26.10.2015 - 12:57:00 \\
\hline
\end{tabular}

\begin{tabular}{|c|}
\hline Vielleicht sind wir ja früher da. Ich halte dir dann einen Platz frei! \\
\hline Nachricht \#5-26.10.2015 - 12:59:00
\end{tabular}

Nachricht \#5 - 26.10.2015 - 12:59:00

Alles klar danke! der der da ist schreibt einfach mal kurz

Nachricht \#6 - 26.10.2015 - 13:05:00

\begin{tabular}{|c|}
\hline Genau \\
\hline Nachricht \#7 $-26.10 .2015-13: 06: 00$ \\
\hline
\end{tabular}

\begin{tabular}{|c|}
\hline Supii bis gleich dann \\
\hline Nachricht \#8 $-26.10 .2015-13: 06: 00$ \\
\hline
\end{tabular}




\begin{tabular}{l}
$\rightarrow \quad$ 3 Plätze zusätzlich oder mit mit zusammen 3 \\
\hline Nachricht \#9 - 26.10.2015 - 14:05:05 \\
$\rightarrow$ Das wird nicht gehen. 2wären noch frei \\
\hline Nachricht \#10 - 26.10.2015 - 14:05:05
\end{tabular}

Die beiden daran beteiligten Schreiberinnen besuchen gemeinsam ein Seminar zur höfischen Literatur und halten in den Nachrichten \#1-2 fest, dass sie sich da sehen werden. Dabei bittet Schreiberin $A$ ihre Kommilitonin, drei Plätze für sie frei zu halten, falls $B$ früher im Seminarraum antreffen soll (\#3). Die Anzahl der frei zu haltenden Plätzen (\#3) wird im späteren Verlauf der Kommunikation (\#9) von $B$ problematisiert, indem sie sich erkundigt, ob ein Platz für sie bei den drei Plätzen inbegriffen ist oder nicht. ${ }^{146}$ Diese Reparaturinitiierung wird allerdings nicht mit einer Reparaturdurchführung eingelöst, weil, wie die zur gleichen Zeit von $A$ verschickte Nachricht zeigt, die Klärung dieser Frage sich erübrigt hat, da lediglich zwei Plätze im Seminarraum noch frei sind.

Darüber hinaus finden sich vereinzelt Fälle, bei denen das Ausbleiben der Reparaturdurchführung mit hoher Wahrscheinlichkeit auf die sequenzielle Organisation der Kurznachrichtenkommunikation sowie auf das Zusammenspiel der sich relativ schnell vollziehenden Rezeption und Produktion zurückzuführen sind. Als Beispiel kann der WhatsApp-Dialog (221) zwischen zwei Freundinnen, die eine Einkaufsliste für einen gemeinsamen Abend zusammenstellen, herangezogen werden:

(221) WhatsApp-Dialog \#4234:

\begin{tabular}{l}
$\rightarrow$ Schreiberin $A$ \\
\hline \\
$\rightarrow \quad$ Welcher Rewe denn? \\
\hline Nachricht \#1 - 09.11.2016 - 17:45:00 \\
\hline Neben der Michael Kirche würde ich sagen...aber erst um 7 nachricht \#2 - 09.11.2016 - 17:46:20 \\
\hline Jap \\
\hline Nachricht \#3 - 09.11.2016 - 17:46:25
\end{tabular}

146 Beim zweiten „mit“ handelt es sich anscheinend um das durch die Autokorrektur veränderte Pronomen mir. 
$\rightarrow$

\begin{tabular}{|c|}
\hline Ähm welche Strasse? \\
\hline Nachricht \#4 - 09.11.2016 - 17:46:35 \\
\hline
\end{tabular}

\begin{tabular}{|c|}
\hline Und was hälst du von Pizza \\
\hline Nachricht \#5 - 09.11.2016 - 17:46:45 \\
\hline
\end{tabular}

Mel hätte gerne Feta drauf was passt da denn noch zu?

Nachricht \#6 - 09.11.2016 - 17:46:50

\begin{tabular}{|c|}
\hline Gut aber ich hab ja keinen Ofen :( \\
\hline Nachricht \#7 - 09.11.2016-17:47:10 \\
\hline
\end{tabular}

$\rightarrow$

\begin{tabular}{|c|}
\hline Ist das der im Zentrum? wo auch der Biomarkt in der nähe ist \\
\hline Nachricht \#8 - 09.11.2016-17:47:20 \\
\hline Oh ehrlich? :) \\
\hline Nachricht \#9-09.11.2016-17:47:40 \\
\hline Dann nicht \\
\hline Nachricht \#10 - 09.11.2016 - 17:47:50 \\
\hline
\end{tabular}

Können ja vllt verschiedene :)ich finde Frischkäse und Hummus beides gut...Cocktailsauce find ich aber auch gut. Ich tue die Wraps immer in der Mikro kurz damit der Käse schmilzt :)wir können sonst auch ein bisschen Hackfleisch machen...dann isst warm...Mel kann ja trotzdem vegetarisch mampfen...Aber wir können auch was ganz anderes machen wenn dir Wraps zu kalt sind :P Nachricht \#11 - 09.11.2016 - 18:21:20

\begin{tabular}{|c|}
$\begin{array}{c}\text { Ähm fehlt dir was anderes warmes ein? Fetakäse wäre nur für die Pizza } \\
\text { gewesen }\end{array}$ \\
\hline Nachricht \#12 - 09.11.2016 - 18:24:25 \\
\hline Chilli sin carne? \\
\hline Nachricht \#13-09.11.2016 - 18:24:40
\end{tabular}

\begin{tabular}{|c|}
\hline Das ist aber doch nicht vegetarisch? \\
\hline Nachricht \#14 - 09.11.2016-18:25:15 \\
\hline
\end{tabular}




\begin{tabular}{|c|}
\hline Sin nicht con ;) \\
\hline Nachricht\#15-09.11.2016-18:25:30 \\
\hline
\end{tabular}

Feta ist aber auch im Wrap geil, mit Avocado :D Was ist denn dann sin carne?

Nachricht \#16 - 09.11.2016 - 18:25:40

\begin{tabular}{|c|}
\hline Zum Beispiel Spaghetti mit Spinat-Feta-Knobi-Sosse \\
\hline Nachricht \#17 - 09.11.2016 - 18:26:05 \\
\hline
\end{tabular}

\begin{tabular}{|c|}
\hline Das wäre einfach und bestimmt lecker \\
\hline Nachricht \#18 - 09.11.2016 - 18:26:15 \\
\hline
\end{tabular}

Ja das klingt auch super :)dann machen wir das Knobi ich sich noch hier Nachricht \#19 - 09.11.2016 - 18:27:20

\begin{tabular}{|c|}
\hline Ok! \\
\hline Nachricht \#20 - 09.11.2016 - 18:27:20 \\
\hline
\end{tabular}

\begin{tabular}{|c|}
\hline Bin da :) \\
\hline Nachricht \#21 - 09.11.2016 - 18:57:15 \\
\hline
\end{tabular}

Nachrichten \#1 bis \#3 lassen sich als Fortsetzung einer Verabredungssequenz interpretieren, deren Anfang in dem vorliegenden Dialog nicht abgebildet wurde, in dem aber eine ReweFiliale vermutlich als Treffpunkt vorgeschlagen wurde. Die in \#1 gestellte Frage soll daher klären, in welcher Filiale sich die beiden Schreiberinnen zum Einkaufen treffen wollen. In diesem Zusammenhang schlägt Schreiberin $B$ eine Filiale neben der Michael-Kirche vor und nutzt außerdem die gleiche Nachricht, um die Zeit des Treffens anzusprechen. B's Vorschlag wird von $A$ zwar mit einem zustimmenden „Jap“ quittiert, der durch die Nähe zu einer Kirche beschriebene Standort der ReweFiliale scheint $A$ jedoch nicht auszureichen, sodass sie in \#4 nähere Informationen einfordert und nach der genaueren Adresse der Filiale fragt („Ähm welche Strasse?“). Der Anfrage nach der genaueren Adresse in \#4, in der mit der Partikel ähm die Mündlichkeit emuliert wurde, folgen in kurzen Abständen zwei weitere Nachrichten: In \#5 wird das Angebot unterbreitet, Pizza zu machen, während in \#6 auf den Wunsch bezüglich des Pizzabelags eingegangen wird. Nachdem in \#7 nicht auf die Reparaturinitiierung in \#4, sondern auf den Vorschlag in \#5 eingegangen wird, unternimmt $A$ in \#8 eine weitere Reparaturinitiierung. Dabei liefert sie das 
candidate understanding ,der im Zentrum? wo auch der Biomarkt in der nähe ist“, das allerdings in der über dreißig Minuten später verschickten Nachricht von $B$ weder bestätigt noch widerlegt wird. Anstatt auf die erneute Reparaturinitiierung einzugehen, scheint Schreiberin $B$ auf die Frage aus \#6 zu reagieren, indem sie Essensvorschläge für den Abend macht. In dem in der Datenbank abgebildeten Verlauf finden sich also weder weitere Reparaturinitiierungen, die den Standort der am Anfang angesprochenen Rewe-Filiale thematisieren, noch eine dieses Problem bearbeitende Reparaturdurchführung. Man kann daher vermuten, dass Schreiberin $A$ das Problem selbst gelöst hat (beispielsweise durch einen Blick auf die Google Maps) oder dass dem vermutlich stattgefundenen Treffen (vgl. \#21) ein Telefonat vorausgegangen ist, sodass der Standort der Filiale mündlich geklärt wurde. Das Ausbleiben der Reparaturdurchführung könnte in diesem Fall dadurch bedingt sein, dass in beiden Fällen die Reparaturinitiierungen in \#4 und \#8 von anderen Nachrichten umgeben sind, in denen unterschiedliche sprachliche Handlungen vollzogen werden (zum Splitting vgl. Beißwenger 2007; Imo 2015a). Eine solche Anordnung der Nachrichten legt daher die Vermutung nahe, dass Schreiberin $B$ entweder die vorliegenden Reparaturinitiierungen übersehen hat oder sie zwar rezipiert hat, aber der Reaktion auf andere Nachricht den Vorrang gegeben hat, wobei ihr die Reparaturdurchführung entging.

\subsubsection{Accounts}

In neun Fällen aus dem deutschen Subkorpus wird das thematisierte Problem nicht gelöst, wobei der Produzent der Problemquelle seine Ratlosigkeit in Bezug auf die von ihm eingeforderte Reparaturdurchführung bzw. seine Unfähigkeit, eine durchzuführen (vgl. Weber 2014: 143-146), zum Ausdruck bringt, was meist in Form von Floskeln wie Weiß ich nicht bzw. Keine Ahnung geschieht:

(222) Auszug aus dem WhatsApp-Dialog \#4131:

Schreiber $A$

Schreiberin $B$

\begin{tabular}{|c|}
\hline Und ich wollte herausfinden, ob das Buch wirklich so schlimm ist, wie \\
alle sagen $\because$ \\
\hline Nachricht \#9 $-11.10 .2016-23: 14$
\end{tabular}

\begin{tabular}{|c|}
\hline Und ist es? \\
\hline Nachricht $\# 10-11.10 .2016-23: 14$ \\
\hline
\end{tabular}




\begin{tabular}{|l|}
\hline Wenn ja dann hör auf zu lesen -.- \\
\hline Nachricht \#11 - 11.10.2016 - 23:15 \\
\hline
\end{tabular}

Ja, ist es. Aber es ist auch schon wieder so schlecht, dass es lustig ist. Besonders, wenn man es kurt vorliest $\Leftrightarrow$

Nachricht \#12 - 11.10.2016 - 23:15

\begin{tabular}{|c|}
\hline Hahaha hör auf 2 \\
\hline Nachricht \#13 - 11.10.2016 - 23:16 \\
\hline
\end{tabular}

\begin{tabular}{|l|}
\hline KT ist auch ohne das Buch witzige \\
\hline Nachricht \#14 - 11.10.2016 - 23:16 \\
\hline
\end{tabular}

\begin{tabular}{|c|}
\hline Manno...nie darf ich $\approx$ \\
\hline Nachricht \#15 $-11.10 .2016-23: 16$ \\
\hline
\end{tabular}

\begin{tabular}{|c|}
\hline Heul nicht \\
\hline Nachricht \#16-11.10.2016-23:21 \\
\hline
\end{tabular}

$\rightarrow$

\begin{tabular}{|c|}
\hline Das ist Diskriminierung! 6 \\
\hline Nachricht \#17 - 11.10.2016 - 23:23 \\
\hline
\end{tabular}

$\rightarrow$

? Was für eine Art von diskriminierung

Nachricht \#18 - 11.10.2016-23:26

$\rightarrow$

\begin{tabular}{|c|}
\hline Keine Ahnung $\Theta$ \\
\hline Nachricht \#19-12.10.2016-07:32 \\
\hline
\end{tabular}

\begin{tabular}{|c|}
\hline $\mathrm{xD}$ \\
\hline Nachricht $\# 20-12.10 .2016-12: 00$ \\
\hline
\end{tabular}

In (222) berichtet Schreiber $A$ darüber, dass er sich entschlossen hat, ein Buch $\mathrm{zu}$ lesen, das von vielen kritisiert wird, um eine eigene Meinung darüber zu bilden. Nachdem Schreiberin $B$ nach $A$ 's Bewertung des Buches fragt, meint er, dass das Buch tatsächlich schlecht sei. Gleichzeitig rechtfertigt $A$ in \#12 das weitere Lesen und erwähnt, dass er dieses Buch einer dritten Person namens Kurt vorliest. Das in \#13 zum Ausdruck gebrachte ,Verbot', das sich vermutlich entweder auf das Lesen oder auf das Vorlesen bezieht, löst bei $A$ eine Reihe von 
Beschwerden aus. Letztendlich weist er seine Kommunikationspartnerin in \#17 darauf hin, dass er sich dadurch diskriminiert fühlt, kann aber die in \#18 eingeleitete Reparaturinitiierung, die auf die Art der Diskriminierung abzielt, nicht einlösen, was er in \#19 offenlegt. Die darauffolgende Reaktion von $B$ in Form des Emoticons „xD“ zeigt, dass die Kommunikation einfach weitergeführt wird.

\subsubsection{Verweigern einer Reparaturdurchführung}

Eine Reparaturinitiierung kann explizit zurückgewiesen werden (ein Fall im russischen und fünf im deutschen Teilkorpus). Das passiert v.a. dann, wenn die Interaktion im Streitmodus erfolgt, wenn eine scherzhafte Aktivität wie ,Frotzeln' etc. ausgeführt wird oder wenn die Reparaturinitiierung als gesichtsbedrohend oder nicht angemessen empfunden wird:

(223) SMS-Dialog \#3894:

Schreiberin $A$

Schreiber $B$

Lieber VORNAME1, meine Veranstaltung morgen von 10-12 fällt aus.

Insofern würde ich gerne unsere Verabredung auf nächste Woche verschieben. Ich hoffe, Du verstehst das, ich würde gerne mit Dir in die Mensa gehen, aber so könnte ich VORNAME2 zu Hause ins Bett legen und an den Schreibtisch gehen. Ok? Lg, VORNAME3

Nachricht \#1 - 17.01.2016 - 20:37

\begin{tabular}{|} 
Liebe VORNAME3, volles Verständnis, erst recht bei den Temperaturen. \\
Kuschelt Euch ein und gib der Süßen bitte einen nachträglichen \\
Geburtstagskuss von mir. Telefonieren wäre vermutlich schwierig \\
geworden. $=$ Liebe Grüße, VORNAME1 \\
\hline Nachricht \#2 $-17.01 .2016-21: 44$ \\
\hline
\end{tabular}

Super. Danke! Die letzte Stunde war ich übrigens damit beschäftigt, SMS von uns in die Datenbank eizuspeisen. Wenn ich richtig gezählt

$\rightarrow$ habe, fehlen noch 5. Du kannst ja mal ein paar garantiert kurze schicken $=$ Nee, Scherz.

Nachricht \#3 - 17.01.2016 - 21:46

$\rightarrow$

Was?

Nachricht \#4 - 17.01.2016 - 21:47 


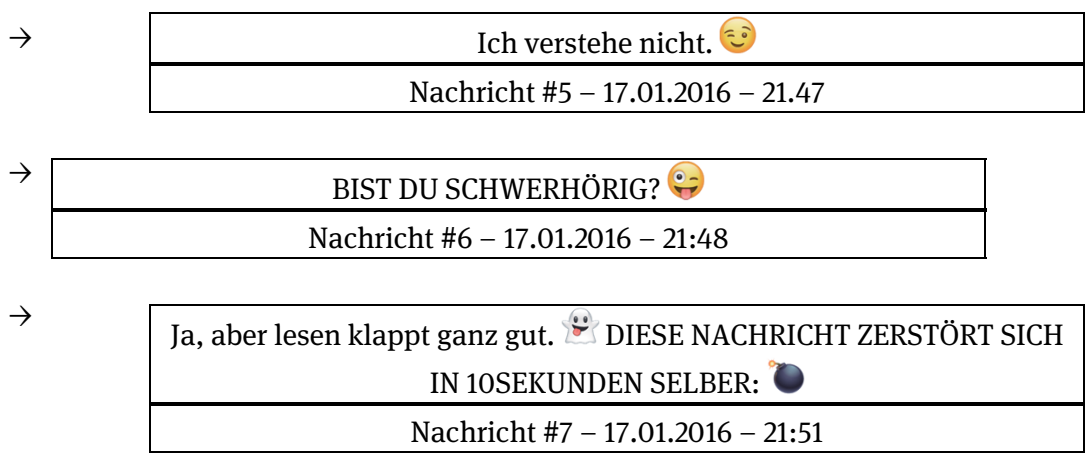

In \#1 äußert Schreiberin $A$ die Bitte, eine Verabredung mit Schreiber $B$ zu verschieben. Als Reaktion darauf stimmt $B$ in Nachricht \#2, die ca. eine Stunde nach dem Eingang der ersten Nachricht verschickt wird, der Verschiebung zu. Anschließend bedankt sich $A$ bei ihrem Kommunikationspartner und berichtet, dass sie während der letzten Stunde SMS-Nachrichten aus den Dialogen, an denen die beiden beteiligt waren, in die MoCoDa eingestellt hat. Dabei erwähnt sie, dass ihr vermutlich noch fünf Nachrichten bis zur für die Leistungserbringung erforderlichen Anzahl von Nachrichten fehlen, und fordert $B$ scherzhaft auf, ihr ein paar kurze Nachrichten zu schicken, wobei sie diese mit dem Emoji ¿- markierte ,Forderung' später explizit zurückweist und als ein Scherz bezeichnet. Die in \#4 sowie in \#5 enthaltenen Reparaturinitiierungen in Form des alleinstehenden Interrogativpronomens „Was?“ sowie der metakommunikativ expliziten Fremdinitiierung „Ich verstehe nicht.“ lassen sich auf zweierlei Weise interpretieren. Einerseits könnte es sich dabei um eine ernst gemeinte Reparaturinitiierung handeln, andererseits könnten sie der in \#3 von $A$ geäußerten Aufforderung geschuldet sein, ein paar Nachrichten mehr zu schreiben, was dazu führen könnte, dass $B$ eine Fremdinitiierung nur dafür einleitet, damit $A$ die benötigte Anzahl an Nachrichten erhält. Für die zweite Interpretation würden beispielsweise das ,zwinkernde“ Emoji in \#5 sprechen sowie die spielerisch gestaltete, scherzhafte Verweigerung der Reparaturdurchführung in \#6, die mit der medialen Realisierung der SMS-Kommunikation spielt (vgl. mediale Schriftlichkeit und Mündlichkeit im Sinne von Koch/Oesterreicher 1985) und die von $B$ in \#7 weitergeführt wird, anstatt die ausbleibende Reparaturdurchführung einzufordern bzw. eine erneute Reparaturinitiierung in Bezug auf die gleiche Problemquelle zu liefern.

\subsubsection{5 ,Auslagerung' einer Reparaturdurchführung}

Des Weiteren kann die Reparaturdurchführung aus der bestehenden Kurznachrichtenkommunikation ausgelagert werden, sodass die Lösung des bestehenden 
Problems im Rahmen eines Face-to-Face-Gesprächs stattfindet (ein Fall in den russischen Daten):

(224) Auszug aus dem SMS-Dialog \#2582:

Schreiber $A$

Schreiberin $B$

\begin{tabular}{|c|}
\hline Тока пришел)го \\
\hline Ich bin eben gekommen)[unklar] \\
\hline Nachricht \#1 - 18.10.2013 - 19:05:05 \\
\hline
\end{tabular}

$\rightarrow \mid$\begin{tabular}{|c|}
\hline че нидь вкумненько вези, у нас ниче нет кушать) \\
\hline Bring was Leckeres mit, wir haben nichts zum Essen) \\
\hline Nachricht \#2 - 18.10.2013 - 19:07:05 \\
\hline
\end{tabular}

У меня нет ничего тоже. Думала, что хоть у тебя есть) Ich habe auch nichts. Ich habe gedacht, dass zumindest du etwas hast)

Nachricht \#3 - 18.10.2013 - 19:09:05

$\rightarrow$

Я могу зайти в магазин. Вкусненького - это что надо купить?

Ich kann einkaufen gehen. Was Leckeres soll ich denn kaufen?

Nachricht \#4 - 18.10.2013 - 19:12:05

$\rightarrow$

\begin{tabular}{|c|}
\hline Давай я тя встречу лучше и там глянем, ты уже где? \\
\hline Lass uns lieber treffen und schauen, wo bist du schon? \\
\hline Nachricht \#5 - 18.10.2013 - 19:23:05 \\
\hline
\end{tabular}

In einem SMS-Dialog zwischen Schreiber $A$ und Schreiberin $B$, die ein Pärchen sind, bittet Schreiber $A$ seine Freundin, etwas Leckeres („че нидь вкумненько“) mitzubringen, woraufhin Schreiberin $B$ erwidert, dass sie nichts Leckeres hat, aber etwas kaufen kann. Anschließend leitet sie eine Reparaturinitiierung in Bezug auf die Problemquelle „че нидь вкумненько“ (,etwas Le-

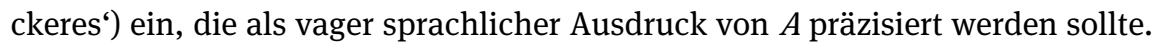
Schreiber $A$ führt aber in \#5 nicht aus, was er in \#2 unter „че нидь вкумненько“ (,etwas Leckeres`) gemeint hat, sondern schlägt vor, zusammen einkaufen zu gehen und dabei face-to-face diese Frage zu klären. 


\subsubsection{Reaktionen auf durchgeführte fremdinitiierte Selbstreparaturen}

Neben den drei wichtigen sequenziellen Komponenten einer Reparatur - der Problemquelle, der Reparaturinitiierung und der Reparaturdurchführung - soll nun kurz auf einen bisher noch nicht systematisch erforschten Aspekt eingegangen werden, nämlich auf die Reaktion des Rezipienten der Problemquelle auf die gelieferte Reparaturdurchführung. In Kap. 6.2.3 wurde bereits darauf hingewiesen, dass eine Reparaturdurchführung, die aus der Sicht des Rezipienten das vorliegende Reparandum nicht adäquat genug bearbeitet, eine weitere Reparaturinitiierung nach sich ziehen kann (vgl. (218)). Des Weiteren kann eine Reparaturdurchführung ein Verstehens- oder Verständigungsproblem lösen, aber gleichzeitig ein anderes auslösen (vgl. \#1977 aus der MoCoDa). In beiden Fällen handelt es sich um Reparaturdurchführungen, die man analog zu Egbert (2009) als (zumindest zum Teil) missglückt bezeichnen könnte, da sie nicht ausreichend zur Wiederherstellung der Intersubjektivität im Sinne von Schegloff (1992) beitragen.

Die Mehrheit der analysierten Fälle bilden jedoch geglückte Reparaturdurchführungen, bei denen der Rezipient der Problemquelle explizit oder implizit sein Verstehen der angebotenen Reparaturdurchführung signalisiert. Die somit erfolgte Verstehensdokumentation (vgl. Deppermann/Schmitt 2008) äußert sich i.d.R. darin, dass die mit der Reparatursequenz unterbrochene sprachliche Handlung wiederaufgenommen und fortgeführt wird (vgl. „Fortsetzung der übergeordneten Gesprächsaktivität“ bei Deppermann/Schmitt 2008: 235). Beispielsweise wird die Verabredungssequenz in (204) fortgesetzt, nachdem die Bezeichnung des von einer Kommunikationsteilnehmerin vorgeschlagenen Ortes (Bar Kliukva) repariert wurde. Ähnlich sieht es in (218) aus, in dem einer der Schreiber seinen Kommunikationspartner an die bevorstehende Verabredung erinnert. Dabei stellt sich im Laufe der Reparatursequenz heraus, dass der Interaktionspartner diese vergessen hat und bereits parallel eine Verabredung mit seiner Freundin vereinbart hat, was zu einer Absage führt.

Abgesehen von dem Fortsetzen der durch die Reparatur ,angehaltenen“ Gesprächsaktivität können Interaktanten ihr Verstehen der dargelegten Reparaturdurchführung verbal zum Ausdruck bringen. Hierfür werden u.a. Responsive wie ja und ok (vgl. (215)) sowie Erkenntnissprozessmarker wie achso oder ach (vgl. (161) und (166)) für das Deutsche und a (,achso') für das Russische (vgl. (212)) verwendet (vgl. dazu ,implizite, kodierte Verstehensdokumentationen' bei Deppermann/Schmitt 2008: 229). Darüber hinaus kann der Rezipient der Problemquelle sein Verstehen der Reparaturdurchführung auch metasprachlich signalisieren (vgl. „explizite Verstehensmanifestationen“ bei Dep- 
permann/Schmitt 2008: 229): In den deutschen Daten finden sich z.B. ,Verstehensmanifestationen“ wie „Okay! Ja das dachte ich mir schon!“ (vgl. (183)) oder „Hab ich mir schon fast gedacht!“ (vgl. (195)), während im Russischen auf das Verb понять (,verstehen') wie in (225) oder auf das davon abgeleitete Adverb понятно ( $\approx$,verstanden') wie in \#2947 zurückgegriffen wird.

In manchen Fällen können metasprachliche ,Verstehensmanifestationen“ zusätzlich eine wichtige Funktion beim face work übernehmen. Eine gesichtswahrende Funktion könnte beispielsweise der ,Verstehensmanifestation“ in Nachricht \#13 aus (225) zugeschrieben werden:

(225) Auszug aus dem WhatsApp-Dialog \#4354:

Schreiberin $A$

Schreiberin $B$

$\rightarrow$

\begin{tabular}{|c|}
\hline В кафе или в Пензе? \\
\hline In einem Café oder in Pensa ${ }^{147}$ ? \\
\hline Nachricht \#8 $-15.02 .18-15: 41$ \\
\hline
\end{tabular}

\begin{tabular}{|c|}
\hline$\Leftrightarrow \Leftrightarrow \theta$ \\
\hline Nachricht \#9-15.02.18 $-15: 41$ \\
\hline
\end{tabular}

$\rightarrow$

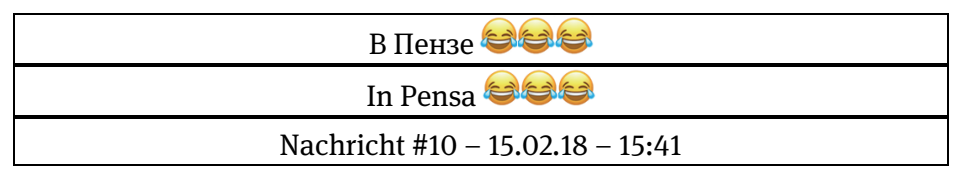

\begin{tabular}{|c|}
\hline В кафе \\
\hline In einem Café \\
\hline Nachricht \#11 - 15.02.18 - 15:42 \\
\hline
\end{tabular}

$\rightarrow$\begin{tabular}{c|}
\hline Мензе \\
\hline Mensa \\
\hline Nachricht \#12-15.02.18-15:42 \\
\hline
\end{tabular}

\begin{tabular}{|c|}
\hline Я поняла $\Theta$ \\
\hline Ich habe verstanden $\Theta$ \\
\hline Nachricht \#13 $-15.02 .18-15: 42$ \\
\hline
\end{tabular}

147 Pensa ist eine Stadt in Russland. 


\begin{tabular}{|c|}
\hline Да я на всякий случай $\oplus$ \\
\hline Ich habe das nur für alle Fälle geschrieben $\Theta$ \\
\hline Nachricht \#14 - 15.02.18 - 15:42 \\
\hline
\end{tabular}

Dem in (225) präsentierten Ausschnitt geht eine ,logistische“ Besprechung voraus, da Schreiberin $A$ sich gleich mit einem Fernbus auf den Weg zu $B$ macht und $B$ sie am Busbahnhof abholen soll. Schreiberin $B$ teilt in diesem Zusammenhang mit, dass sie gerade eine Mittagspause macht und in Kürze zum vereinbarten Treffpunkt aufbrechen wird. Danach fragt $A$, ob $B$ in einem Café oder in der Mensa zu Mittag isst. Dabei verschickt sie aber anstatt des intendierten $B$ мензе ${ }^{148}$ (,in der Mensa') eine andere Präpositionalphrase, nämlich „в Пензе“ (,in Pensa'), wobei Pensa der Name einer russischen Stadt ist. Die darauffolgende Kombination aus drei Emojis , könnte zum einen eine Art Kommentarfunktion (vgl. Pappert 2017: 196-198) übernehmen, mit der $A$ signalisiert, dass sie ihren Fehler entdeckt hat, und Stellung dazu nimmt. Zum anderen fungieren Emojis in dem Fall als Ressourcen, die die für $A$ gesichtsverletzende Handlung, nämlich einen Fehler, der ihr unterlaufen ist, modalisieren und somit zur Wahrung ihres Gesichts beitragen (vgl. Imo 2015b: 150-152; Pappert 2017: 195-196). Auf die in \#8 gestellte Frage geht $B$ ein, indem sie zuerst in \#10 A’s Fehler aufgreift und mit drei ,tränenlachenden' Emojis versieht, die in dem Fall polyfunktional sind. Zum einen markieren sie die scherzhafte Modalität der Nachricht (vgl. Imo 2015b: 148-150; Pappert 2017: 195-196), womit die für $A$ gesichtsbedrohende Wirkung der gesamten Nachricht \#10 abgeschwächt wird (vgl. Imo 2015b: 150-152). Zum anderen bringen die drei Emojis B's Bewertung des ihrer Kommunikationspartnerin unterlaufenen Fehlers ins Spiel (vgl. die Funktion Evaluierung/Kommentierung in Pappert 2017: 196-198). Erst eine Nachricht später, und zwar in \#11, liefert $B$ eine angemessene Antwort auf $A$ 's Frage, und zwar „В кафе“ (,In einem Café‘), sodass die Kommunikation weiter fortgesetzt werden kann. Allerdings reagiert $A$ auf \#10, die von $B$ eigentlich nicht als Fremdinitiierung gedacht wurde (vgl. \#13), mit einer Reparaturdurchführung „Мензе“ (,Mensa)). Dabei trägt diese Reparaturdurchführung nicht unbedingt zur Verstehenssicherung bei, da $B$ bereits auf die das Reparandum enthaltende Nachricht angemessen reagiert hat, sodass die Reparaturdurchführung keine kommunikative Relevanz mehr hat und streng genommen hinfällig ist. Diese scheint vielmehr für das face work von Relevanz zu sein: Durch das

148 An der Stelle muss angemerkt werden, dass das Wort менза kein russisches Wort ist, sondern eine Transliteration des deutschen Wortes Mensa darstellt. Daher könnte man dieses als ein Beispiel für ein Code-Switching ohne Script-Switching (vgl. Ma i.Dr.) ansehen. 
Verschicken der intendierten Präpositionalphrase zeigt sich $A$ als kompetente Schreiberin und schützt dadurch ihr eigenes Gesicht. Die in \#12 erfolgte Reparaturdurchführung führt jedoch dazu, dass $B$ im Gegenzug gezwungen wird, ihr Gesicht zu schützen, da ihr mit \#12 implizit unterstellt werden könnte, dass sie aus der in \#8 vorliegenden Phrase „в Пензе“ (,in Pensa`) die richtige Version $\boldsymbol{B}$ мензе (,in der Mensa') nicht rekonstruieren konnte. Diese für sie potenziell gesichtsbedrohende Unterstellung federt sie daher mit \#13 ab, indem sie explizit ihr Verstehen des in der Nachricht \#8 gemeinten Ausdrucks bestätigt. Darauf reagiert $A$ mit der Anmerkung, dass ihre Reparaturdurchführung eher als eine Art ,Vorsorge‘ zu verstehen ist, sodass \#14 ebenso eine gesichtswahrende Funktion ausübt, wobei in diesem Fall sowohl das eigene als auch das fremde Face geschützt wird.

Außer den bereits oben diskutierten Optionen, auf eine Reparaturdurchführung zu reagieren, wurde in den Analysedaten eine weitere Strategie gesichtet, die ähnlich wie die Fortsetzung der ,angehaltenen' sprachlichen Aktivität nicht auf der sprachlichen Oberfläche das Verstehen signalisiert, dabei aber explizit auf den Inhalt der Reparaturdurchführung selbst eingeht. $\mathrm{Zu}$ dieser Gruppe werden Fälle gezählt, in denen der Rezipient der Problemquelle eine Bewertung oder einen Kommentar zur vorgelegten Reparaturdurchführung liefert. Dabei kann sich eine solche Reaktion aus einem verbalen Teil und einem Emoticon bzw. Emoji zusammensetzen (vgl. „Kanns mir bildlich vorstellen wie du ins Telefon brüllst :-)“ in (210) oder „Boa richtiger Rebell 30 “ “ in (188)). In einigen Fällen wird diese Aufgabe nur von Emoticons, Emojis oder sogar Lachpartikeln übernommen (vgl. „xD“ in (222) sowie „haha“ in (160)).

\subsubsection{Emoticons und Emojis bei fremdinitiierten Selbstreparaturen}

Bei der Analyse einiger Beispiele in Kapitel 6.2 wurde bereits vereinzelt auf den Gebrauch von Emoticons und Emojis eingegangen. Im vorliegenden Kapitel soll nun ein systematischerer Überblick über die Verteilung von Emoticons und Emojis im Analysekorpus gegeben werden. Zudem wird im Folgenden auf einige Funktionen von Emoticons und Emojis im Kontext von fremdinitiierten Selbstreparaturen eigegangen. Die Auswertung der Daten hat gezeigt, dass Emoticons und Emojis zum einen selbst ein interaktionelles Problem auslösen (vgl. (189) und (208)), zum anderen aber im Rahmen einer Nachricht mit dem Reparandum als Kontextualisierungshinweis genutzt werden können, um eine candidate solution für die Problemquelle anzubieten (vgl. (203)). Im Folgenden liegt das Augenmerk v.a. auf dem Vorhandensein bzw. dem Ausbleiben von 
Emoticons und Emojis in solchen sequenziellen Bestandteilen einer Reparatur wie der Reparaturinitiierung und der Reparaturdurchführung.

\subsubsection{Emoticons und Emojis bei Reparaturinitiierungen}

\section{Formale Aspekte}

Alle Fremdinitiierungen im deutschen und russischen Korpus wurden auf das Vorhandensein eines oder mehrerer Emoticons und Emojis überprüft. Es hat sich herausgestellt, dass sowohl im russischen als auch im deutschen Subkorpus jeweils ca. 80\% der Fremdinitiierungen keine Emoticons oder Emojis beinhalten: In 271 Fremdinitiierungen in den deutschen Kurznachrichten finden sich insgesamt lediglich 52 Emoticons und Emojis, während bei russischen Fremdinitiierungen nur in 17 von 85 Fällen Emoticons und Emojis gebraucht werden. ${ }^{149}$

Darüber hinaus wurden auch solche Phänomene, die sich u.U. funktional mit dem Gebrauch von Emoticons und Emojis überschneiden könnten, gesondert gezählt. Dazu gehören z.B. Lachpartikeln wie haha, die dreimal im deutschen Korpus vorkommen, sowie das Akronym лол (,lol'), das in einem russischen Dialog auftritt.

Die Tatsache, dass Emoticons und Emojis in lediglich ca. 20\% der Fremdinitiierungen in der jeweiligen Sprache vorkommen, könnte mit mehreren Faktoren zusammenhängen. Zum einen könnte das Ausbleiben von Emoticons und Emojis durch die Produktionsbedingungen einer Fremdinitiierung im Rahmen informeller interaktioneller Schriftlichkeit beeinflusst werden. Im Laufe einer medial schriftlichen Interaktion per SMS oder WhatsApp muss der Rezipient der Problemquelle im Idealfall möglichst zügig eine Fremdinitiierung liefern, damit diese von dem Produzenten der Problemquelle dem entsprechenden Reparandum zugeordnet und anschließend bearbeitet werden kann, was dem Entstehen weiterer Verstehens- und Verständigungsprobleme vorbeugt. Da aber die Produktion und Rezeption der Beiträge im Falle einer schriftlichen Interaktion voneinander entkoppelt sind, kann der Produzent der Problemquelle nicht wissen, dass sich sein Kommunikationspartner gerade mit dem Formulieren einer Fremdinitiierung beschäftigt, und kann deswegen u.U. weitere Nachrichten verschicken (Stichwort: Mühlenprinzip im Sinne von Wichter 1991). Das Eintippen eines Emoticons oder eines Emojis auf einem Smartphone erfordert das Umschalten zur Tastatur mit Interpunktionszeichen oder das Aufrufen der

149 Vgl. Fußnote 125. 
,Emojis-Liste‘. Im ersten Fall müssen mindestens zwei Tasten mit Interpunktionszeichen getätigt werden, während im zweiten Fall das benötigte Emoji aus einer Liste ausgesucht werden muss. Die Eingabe eines Emoticons oder eines Emojis mithilfe einer ,physischen‘ Tastatur eines Handys gestaltet sich ebenfalls ziemlich mühsam. Der Verzicht auf Emoticons und Emojis spart somit Zeit und steigert die Chancen, die Fremdinitiierung möglichst kurz zu halten und schnell abzuschicken.

Die zweite mögliche Erklärung hängt nicht mit der medialen Realisierung einer Fremdinitiierung, sondern mit der Natur des Phänomens an sich zusammen. Meines Wissens liegen zurzeit keine systematischen Untersuchungen zur multimodalen Analyse von Fremdinitiierungen vor, sodass es nicht ausgeschlossen werden kann, dass viele Fremdinitiierungen in der gesprochenen Sprache ebenso ohne begleitende para- und nonverbale Mittel auskommen. Ausgenommen sind natürlich Fälle, in denen eine nicht-ernsthaft gemeinte Fremdinitiierung prosodisch markiert oder beispielsweise durch ein Lachen begleitet wird.

Abgesehen davon, dass Emoticons und Emojis lediglich jeweils in ca. 20\% aller Reparaturinitiierungen vorkommen, findet sich in den beiden Korpora eine relativ breite Palette an unterschiedlichen Emoticons und Emojis - 18 Types in den deutschen und sieben in den russischen Daten, wobei in den deutschen Kurznachrichten das Emoticon :D(14 Tokens) und in den russischen das Emoticon :-) und seine Varianten (acht Tokens) im Vergleich $\mathrm{zu}$ anderen Emoticons und Emojis am häufigsten vertreten sind.

\section{Funktionale Aspekte: Emojis als Fremdinitiierungen}

Aus funktionaler Perspektive lässt sich festhalten, dass Emoticons und Emojis in den untersuchten Reparaturinitiierungen eine Reihe von interaktionalen Aufgaben übernehmen. Die erste und die für die vorliegende Arbeit zentrale Funktion besteht darin, dass alleine stehende Emoticons und Emojis als Fremdinitiierungen fungieren können. Im Untersuchungskorpus wird von dieser Funktion jedoch äußerst selten Gebrauch gemacht: Es findet sich lediglich je ein Fall im deutschen und russischen Korpus (vgl. die Analyse von (226) und (227)), bei dem eine mit dem Emoji mittransportierte Bedeutung bzw. Einstellung im gegebenen Kontext den Rezipienten zur Bearbeitung seiner letzten Nachricht bringt.

Zuerst soll ein Beispiel aus einem deutschen Kurznachrichtendialog vorgestellt werden, in dem während der Interaktion zwischen Schreiber $A$ und Schreiberin $B$ zwei Frauennamen erwähnt werden - Johanna (Nachricht \#17) und Gerbi (Nachricht \#19). Dabei scheint Schreiber $A$ mit Johanna eine romanti- 
sche Beziehung anzustreben, während seine Beziehung zu Gerbi aus dem Kontext nicht ersichtlich ist:

(226) Auszug aus dem Dialog \#4273:

Schreiber $A$

Schreiberin $B$

\begin{tabular}{|c|}
\hline Neue Nachrichten um Johanna? \\
\hline Nachricht \#17 - 06.12.2016 - 20:58:05 \\
\hline
\end{tabular}

Nicht wirklich. Wir schreiben jeden Tag und ja.. das ist es schon..

Nachricht \#18 - 06.12.2016 - 20:59:05

Eigentlich wollte ich mich ja heute mit Gerbi treffen, aber die hatte heute doch keine Zeit mehr $\wedge \wedge$

Nachricht \#19 - 06.12.2016 - 20:59:05

\begin{tabular}{|c|}
\hline$:)$ \\
\hline Nachricht \#20-06.12.2016-21:47:05 \\
\hline
\end{tabular}

\begin{tabular}{|c|}
\hline Aufstellung? \\
\hline Nachricht \#21 - 06.12.2016- 21:47:05 \\
\hline
\end{tabular}

\begin{tabular}{|c|}
\hline Jupp \\
\hline Nachricht \#22 - 06.12.2016- 21:48:05 \\
\hline
\end{tabular}

\begin{tabular}{|c|}
\hline Ich bin gespannt...ich hoffe es klappt bei dir :) \\
\hline Nachricht \#23-06.12.2016 - 21:48:05 \\
\hline
\end{tabular}

$\rightarrow$

Meine Meinung, Hoffnungen und Vorstellungen dazu, ändern sich stündlich 8

Nachricht \#24 - 06.12.2016 - 21:49:05

$\rightarrow$

$\mathrm{Zu}$ Johhana oder Gerbi?

Nachricht \#25 - 06.12.2016 - 21:54:05

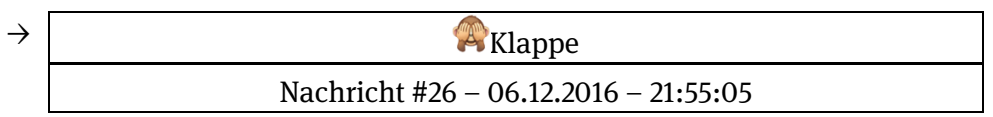




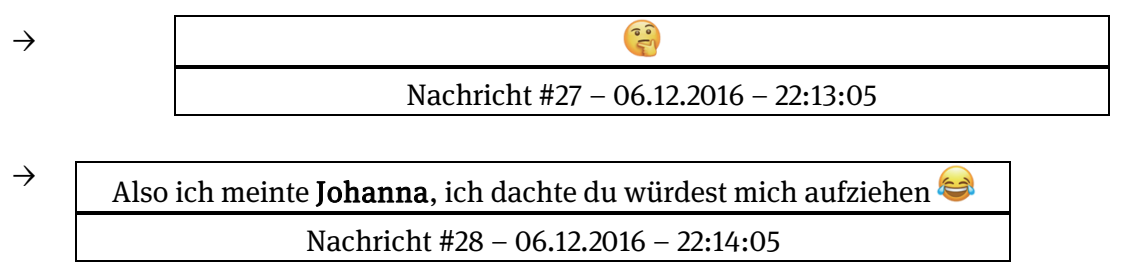

\begin{tabular}{|c|}
\hline Ist ja auch so ;) \\
\hline Nachricht \#29-06.12.2016-22:18:05 \\
\hline
\end{tabular}

\begin{tabular}{|c|}
\hline Pff \\
\hline Nachricht \#30-06.12.2016 - 22:18:05 \\
\hline
\end{tabular}

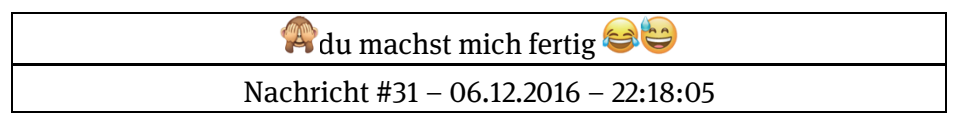

In \#24 teilt $A$ mit, dass seine Einstellungen „dazu“ sich ständig ändern, ohne jedoch die mit dem deiktischen Adverb gemeinte Objektreferenz (also, seine Beziehung zu Gerbi oder zu Johanna) zu präzisieren. $B$ nimmt den problematischen Ausdruck „dazu“ als Anlass für eine Fremdinitiierung, die allerdings nicht auf eine Objektreferenz, sondern auf eine Personenreferenz bezieht, indem sie die beiden zuvor erwähnten Frauennamen als candidate references in \#25 anbietet (,Zu Johhana oder Gerbi?“). Diese Fremdinitiierung wird von Schreiber $A$ jedoch als eine Frotzelaktivität wahrgenommen, sodass er darauf nicht mit einer dazu passenden Reparaturdurchführung, sondern mit einer Zurückweisung der Nachricht \#25 reagiert (\#26). Das darauffolgende Emoji mit Hand am Kinn : aus \#27 könnte als Ausdruck von B's Erwartungsproblem interpretiert werden, das durch $A$ 's Reaktion auf ihre Fremdinitiierung ausgelöst wurde. Dieses mag $A$ den Eindruck vermittelt haben, dass seine Lesart der Fremdinitiierung falsch war, sodass deren Zurückweisung in \#26 Schreiberin $B$ gegenüber womöglich gesichtsbedrohend und nicht gerechtfertigt ist. Daher liefert $A$ in \#28 doch die ursprünglich verweigerte Reparaturdurchführung, die eine der von $B$ angebotenen candidate references bestätigt. Zudem rechtfertigt $A$ seine Reaktion in \#26, indem er seine Interpretation von $B$ 's Fremdinitiierung als eine Verspottung offenlegt, was von $B$ in \#29 allerdings bestätigt wird.

Das zweite Emoji, dem eine reparative Funktion zugeschrieben werden kann, taucht ebenfalls in einem scherzhaften Kontext auf: 
(227) Auszug aus dem Viber-Dialog \#3122:

Schreiberin $A$

Schreiber $B$

\begin{tabular}{|c|}
\hline Угадай где я сегодня была! \\
\hline Rate mal, wo ich heute war! \\
\hline Nachricht \#1 - 18.02.2014 - 14:05:05 ${ }^{150}$ \\
\hline
\end{tabular}

$\rightarrow$

\begin{tabular}{|c|}
\hline У президента в туалете?) \\
\hline In der Toilette eines Präsidenten?) \\
\hline Nachricht \#2 - 18.02.2014-14:05:05 \\
\hline
\end{tabular}

$\rightarrow$

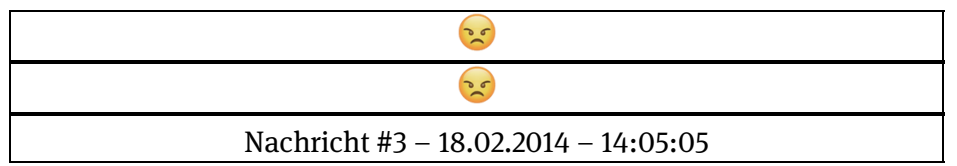

$\rightarrow$

\begin{tabular}{|c|}
\hline Ладно шутка)) большой театр?)) \\
\hline Okay, okay, das war nur ein Witz)) Bolschoi-Theater?)) \\
\hline Nachricht \#4 - 18.02.2014 - 14:05:05 \\
\hline
\end{tabular}

\begin{tabular}{|c|}
\hline Даaаaаaaaa \\
\hline Jaaaaaaaaa \\
\hline Nachricht \#5 - 18.02.2014 - 14:05:05 \\
\hline
\end{tabular}

Schreiberin $A$ lädt ihren Kommunikationspartner in (227) ein zu raten, wo sie an jenem Tag war. Schreiber $B$ reagiert darauf mit einer nicht ernsthaft gemeinten Antwort (\#2), deren Dispräferenz sowie die Dispräferenz des Witzes an sich Schreiberin $A$ durch das Verschicken eines, wütenden' Emoji $\sigma$ of (\#3) signalisiert. $A$ 's Reaktion auf seinen Witz bringt $B$ dazu, die Interaktionsmodalität zu wechseln: Dabei stuft $B$ seine Antwort in \#4 explizit als einen Witz ein, nimmt diesen zurück und ersetzt ihn durch eine andere, ernsthafte Hypothese „большой театр“ (,Bolschoi-Theater'), die von $A$ bestätigt wird.

Die beiden oben als Reparaturinitiierungen interpretierten Emojis (vgl. (226) und (227)) erscheinen somit in einem - zumindest für einen Interaktionspartner - gesichtsbedrohenden Kontext und führen dazu, dass der Produzent der Problemquelle die Modalität seiner Äußerung ändert bzw. explizit rechtfertigt (vgl. face work im Sinne von Goffman 1967). Da, wie (226) und (227) zeigen, Emojis in

150 Vgl. Fußnote 117. 
ihrer reparativen Funktion im Stande sind, die Modalität der Äußerung als Problemquelle zu markieren, könnten diese als eine Art Ökonomisierungsstrategie im Gegensatz zu verbal formulierten Fremdinitiierungen, die auf ähnliche Problemquellen gerichtet sind, angesehen werden (zur kommunikativen Funktion der Ökonomisierung bei Emojis vgl. Pappert 2017: 191-192). Damit tragen Emojis zur Beschleunigung der Nachrichtenproduktion sowie zur „Verdichtung dialogischer Strukturen“ (Androutsopoulos 2007: 78) bei.

In Bezug auf die Zuordnung von Emojis zu einem der in der vorliegenden Arbeit beschriebenen Initiierungsformate lassen sich wegen der geringen Anzahl an Beispielen noch keine validen Aussagen treffen. Da Emojis alleine keinen expliziten Bezug zur Problemquelle (z.B. in Form einer Teilwiederholung) aufbauen können, stehen sie vermutlich unspezifischen Fremdinitiierungen nahe. Zugleich stellt sich aber die Frage, ob Emojis als Fremdinitiierungen auch bei solchen Fällen eingesetzt werden können, in denen nur ein Teil der Nachricht (und nicht die ganze Nachricht wie in (226) und (227)) als problematisch gilt. Zudem bleibt auch die Frage nach potenziellen Problemquellen offen, die Emojis anzeigen können: Kann mit Emojis nur die in der gegebenen Kommunikationssituation als nicht-angemessen bewertete Modalität problematisiert werden oder können Emojis auch auf andere Problemtypen wie z.B. auf Referenzprobleme verweisen? Diesen Forschungsfragen muss in zukünftigen Untersuchungen nachgegangen werden.

\section{Funktionale Aspekte: ,Nicht-reparative‘ Funktionen von Emojis und Emoticons}

Abgesehen von der reparativen Funktion üben Emoticons und Emojis eine Reihe weiterer Funktion aus: In Anlehnung an die von Pappert (2017) für den Gebrauch von Emojis herausgearbeiteten Funktionen kann festgehalten werden, dass in den untersuchten Reparaturinitiierungen Emojis und Emoticons v.a. in drei Funktionen eingesetzt werden, nämlich zur Rahmung eines Dialogs als privater informeller Interaktion (vgl. auch die Verwendung von :-) zum Markieren von phatischer Kommunikation bei Imo 2015b: 146-148; vgl. \#4274 in der MoCoDa), zur Modalisierung einer Fremdinitiierung (vgl. (182) sowie Nachricht \#40 in \#4381 aus der MoCoDa) sowie zur Kommentierung/Evaluierung der Problemquelle bzw. der Fremdinitiierung (vgl. (188), (200), (203) sowie (225)). Zudem finden sich vereinzelt Fälle, in denen Emoticons und Emojis der Strukturierung 
einer Nachricht dienen, zur Beziehungsgestaltung ${ }^{151}$ zwischen den Interagierenden oder zur ikonischen Darstellung ${ }^{152}$ einzelner Gegenstände, die in der Nachricht erwähnt werden, beitragen (vgl. Pappert 2017). Dabei muss darauf hingewiesen werden, dass bei einigen Reparaturinitiierungen mehrere Funktionen gleichzeitig zutreffen können.

\subsubsection{Emoticons und Emojis bei Reparaturdurchführungen: Formale und funktionale Aspekte}

Im Vergleich zu Reparaturinitiierungen werden Emoticons und Emojis bei Reparaturdurchführungen etwas häufiger gebraucht: Während in den deutschen und russischen Fremdinitiierungen jeweils in ca. 20\% der Fälle Emoticons und Emojis zu finden sind, kommen diese im deutschen Teilkorpus in 38,5\% und im russischen in 33,3\% aller Nachrichten mit Reparaturdurchführungen vor. Das häufigere Vorkommen von Emoticons und Emojis bei Reparaturdurchführungen kann damit zusammenhängen, dass Produzent der Problemquelle u.U. im Gegensatz zum Rezipienten der Problemquelle unter einem geringeren Zeitdruck steht, da die Reparaturdurchführung bereits durch eine vorliegende Fremdinitiierung konditionell relevant gemacht wurde, sodass sich der Rezipient bereits darauf eingestellt hat und sogar evtl. vom Verschicken weiterer Nachrichten absieht, bevor die Reparaturdurchführung nicht geliefert wird. Eine andere Begründung für die quantitative Verteilung von Emoticons und Emojis in den analysierten Reparaturdurchführungen könnte darin liegen, dass fremdinitiierte Selbstreparaturen als (potenziell) gesichtsbedrohende sprachliche Handlungen angesehen werden können, und zwar sowohl für Produzenten als auch für Rezipienten der Problemquelle. Emoticons und Emojis können dabei als Modalisierungsressourcen auftreten und somit zur Wahrung des Gesichts der beiden Kommunikationsteilnehmer beitragen.

In formaler Hinsicht lassen sich in den beiden Teilkorpora weitgehend die gleichen Emoticons und Emojis wie bei Fremdinitiierungen beobachten: Dazu gehören z.B. die Emoticons $: D$, :) ${ }^{153}$,;) und das Emoji $\Theta_{-}$. Dabei fällt auf, dass

151 Diese Funktion kann beispielsweise dem Emoticon $<3$ in der Fremdinitiierung ,Jaa kann ich dir gleich erzählen wenn du möchtest :) Was heißt ganz ok soweit? <3“ aus (219) zugeschrieben werden.

152 In \#4246 wird mithilfe einer Reparaturinitiierung, die mit dem Emoji $\bigcirc$ versehen ist, der Name eines Kuchens erfragt.

153 Varianten des ,lächelndes‘ Emoticons wie :-), :) sowie die in der russischen CMC verbreitete ,elliptische‘ Form ), die lediglich aus einer Klammer besteht, wurden zusammengezählt. Dasselbe gilt auch für iterierte Formen wie :))oder ))). 
bei Reparaturdurchführungen ebenfalls die Emoticons :D (22 Tokens) sowie :-) (14 Tokens) jeweils den ersten Platz in den deutschen und russischen Daten belegen (vgl. Kap. 6.2.5.1).

Neben der modalisierenden Funktion (vgl. (184) und \#4239 in der MoCoDa; vgl. Imo 2015b: 150-152; Pappert 2017: 195-196) üben Emoticons und Emojis in den analysierten Reparaturdurchführungen auch Funktionen wie Rahmung (vgl. \#4274), Evaluierung/Kommentierung (vgl. \#2455) sowie - lediglich in einem Fall - Darstellung (das Emoji im Kontext einer Nachricht über Weihnachten, vgl. \#4246 in der MoCoDa) aus.

\subsubsection{Interaktionale Funktionen von Fremdinitiierungen}

Die primäre Funktion von Fremdinitiierungen besteht darin, dem Rezipienten eine Ressource zur Verfügung zu stellen, mit der er seinem Kommunikationspartner signalisieren kann, dass er mit einer kompletten Nachricht oder mit einem oder mehreren ihrer Teile ein Problem hat. Dieses soll dann im Laufe der darauffolgenden Reparaturdurchführung vom Produzenten der Problemquelle bearbeitet werden, was zur Wiederherstellung der Intersubjektivität führen soll (vgl. Stukenbrock 2013: 242). Auf diese Funktion von Fremdinitiierungen wird u.a. in den Fällen zurückgegriffen, in denen (zumindest aus der Sicht des Rezipienten) ein abrupter Themenwechsel oder eine „sequenzielle Entgleisung“ vorliegt (vgl. Egbert 2009: 118-120; für den Gebrauch von unspezifischen Initiierungsformaten in diesen interaktionalen Kontexten im Englischen vgl. Drew 1997).

Das interaktionale Potenzial von Fremdinitiierungen beschränkt sich allerdings nicht darauf, zur Verstehensaushandlung beizutragen. Parallel $\mathrm{zu}$ ihrer primären Funktion können Fremdinitiierungen auch andere Funktionen in der Interaktion ausüben. Beispielsweise zeigt Egbert (2009: 120-121), dass Fremdinitiierungen ein effizientes Mittel für das „Einklinken“ in ein bereits laufendes Gespräch darstellen. Darüber hinaus kann mit Fremdinitiierungen die Gruppenstruktur bei einem Mehrparteien-Gespräch geändert werden, und zwar ein gemeinsames Gespräch kann in zwei parallel zueinander laufende Interaktionen aufgesplittet werden (,Schisma-Bildung“ oder „schisming inducing“ bei Egbert 2009: 121-122). Umgekehrt können zwei Gespräche wieder ,zusammengeführt` werden („Schisma-Verfall“ oder „merging“ bei Egbert 2009: 121-122).

Für das Englische merkt Kendrick (2015: 181-187) an, dass in seinen Daten neben ,reinen“ Fremdinitiierungen (,genuine other-initiations of repair“) auch Fremdinitiierungen vorhanden sind, die primär eine andere Funktion erfüllen. 
Dabei können Fremdinitiierungen laut Kendrick (2015: 181-187) folgende Funktionen übernehmen: (i) für scherzhafte Aktivitäten (Kendrick 2015: 181: „nonserious actions, such as jokes and teases“) eingesetzt werden; (ii) einer dispräferierten Antwort vorausgehen (Kendrick 2015: 181: „preliminaries to dispreferred responses“; vgl. dazu auch Schegloff/Jefferson/Sacks 1977: 380) und (iii) die Überraschung oder den Zweifel des Rezipienten anzeigen (Kendrick 2015: 181: „displays of surprise and disbeliefs“).

In den analysierten Kurznachrichtendialogen erfüllen die meisten Fremdinitiierungen ausschließlich ihre primäre Funktion: Sie signalisieren das Vorhandensein einer Problemquelle in der Nachricht des Kommunikationspartners. Vereinzelt finden sich jedoch Beispiele, in denen Fremdinitiierungen auch gleichzeitig als Ressource für die von Kendrick (2015) beschriebenen Handlungen eingesetzt werden. Darüber hinaus können einzelne Fremdinitiierungen auch als (getarnte) Frotzelaktivitäten (vgl. Günthner 1996, 1999) fungieren (vgl. (226)) bzw. eine dispräferierte Antwort ankündigen (vgl. (162)).

Zudem kommen einige Fremdinitiierungen sowohl im deutschen als auch im russischen Subkorpus im Kontext von Streitsequenzen vor und werden neben ihrer Primärfunktion dafür eingesetzt, einen gegen den Rezipienten der Problemquelle gerichteten Vorwurf abzuwehren (zum Gebrauch der Fremdinitiierungen bei Konflikten vgl. Egbert 2009: 120-122). Ein deutschsprachiges Beispiel, in dem Schreiberin $B$ ihrer Schwester unterstellt, kein Recht auf eine Frage nach ihrer Ankunftszeit zu stellen, wurde bereits in (217) vorgestellt. Diese Unterstellung wird von Schreiberin $A$ durch die darauffolgende Fremdinitiierung hinterfragt und gleichzeitig zurückgewiesen. Für das Russische kann der Auszug (228) aus einem WhatsApp-Dialog zur Veranschaulichung herangezogen werden:

(228) Auszug aus dem WhatsApp-Dialog \#4348:

\begin{tabular}{|c|c|}
\hline \multicolumn{2}{|c|}{ Schreiberin $A$} \\
\hline & Ну всё Второй косяк за неделю уже \\
\hline & Das war nun ja bereits dein zweites, Verschulden' diese Woche \\
\hline & Nachricht \#6 - 08.11.2014 - 14:05:05 $5^{154}$ \\
\hline
\end{tabular}

154 Vgl. Fußnote 117. 


\begin{tabular}{|c|}
\hline $\begin{array}{c}\text { В смысле? Что-то кто-то мне настроение подпарчивает своими } \\
\text { непонятными мне косяками }\end{array}$ \\
\hline $\begin{array}{l}\text { Was soll das denn heißen? Jemand scheint mir die Stimmung mit } \\
\text { seinen unverständlichen,Verschulden` zu verderben }\end{array}$ \\
\hline Nachricht \#7 - 08.11.2014 - 14:05:05 \\
\hline ну чо ты шляешься с кем-то \\
\hline Nun, du treibst dich doch mit was weiß ich wem herum \\
\hline Nachricht \#8 - 08.11.2014 - 14:05:05 \\
\hline
\end{tabular}

\begin{tabular}{|c|}
\hline в смысле с кем-то? Ой всё,короче \\
\hline Was heißt mit was weiß ich wem? Ach, Schluss damit \\
\hline Nachricht \#9 - 08.11.2014 - 14:05:05 \\
\hline
\end{tabular}

\begin{tabular}{|c|}
\hline хахахаха,ты так смешно злишься,[имя]: ${ }^{\star}$ \\
\hline hahahaha, du bist so lustig, wenn du dich ärgerst [Name]: ${ }^{\star}$ \\
\hline Nachricht \#10 - 08.11.2014 - 14:05:05 \\
\hline
\end{tabular}

Im vorherigen Verlauf des Dialogs bittet Schreiberin $A$ ihren Freund, sie nach einem Treffen mit ihren Kommilitonen abzuholen. Darauf reagiert Schreiber $B$ mit einem Vorwurf, dass das ihr zweites ,Verschulden' in der laufenden Woche sei. Auf den in \#6 von $B$ geäußerten Vorwurf geht $A$ zuerst mit einer Fremdinitiierung „В смысле?“ (,In welchem Sinne? Was soll das denn heißen?') ein, mit der sie ihr Nicht-Einverständnis mit dem vorangehenden Vorwurf offenlegt. Anschließend liefert sie einen Gegenvorwurf. Die von $B$ in \#8 verschickte Reparaturdurchführung präzisiert den in \#6 dargelegten Vorwurf und führt zu einem weiteren Erwartungsbruch bei $A$, welchen sie zwar in \#9 thematisiert, gleichzeitig aber zeigt, dass sie an der entsprechenden Reparaturdurchführung nicht interessiert ist, sondern für das Beenden des Streites plädiert.

\subsubsection{Fremdinitiierte Selbstreparaturen im Kontrast}

\subsubsection{Fremdinitiierte Selbstreparaturen in der gesprochenen Sprache und in der Kurznachrichtenkommunikation}

Aus der Datenanalyse in 6.2.1-6.2.6 lässt sich schlussfolgern, dass fremdinitiierte Selbstreparaturen in der Kurznachrichtenkommunikation eine Reihe von Gemeinsamkeiten sowie Unterschieden mit fremdinitiierten Selbstreparaturen in der gesprochenen Sprache aufweisen. Einige Überschneidungen zwischen 
Reparaturen in der medialen Mündlichkeit und Schriftlichkeit finden sich beispielsweise bei einzelnen Typen von Problemquellen, die eine Fremdinitiierung notwendig machen. Sowohl in einem mündlichen Gespräch als auch in einem Kurznachrichtendialog kann es z.B. durch den Gebrauch eines deiktischen Ausdrucks zu einem Referenzproblem kommen (vgl. (28) und (29) für die gesprochene Sprache sowie (183) und (185) für die Kurznachrichtenkommunikation). Ähnlich sieht es mit Bedeutungsverstehensproblemen aus, von denen viele, wie in (167) und (184), auch in der gesprochenen Sprache denkbar wären. Das Gleiche gilt für einige im Korpus vertretene Erwartungsprobleme (vgl. (212)).

Neben Problemquellen lassen sich bestimmte Parallelen im Bereich der Initiierungsformate beobachten. Sowohl in der gesprochenen als auch in der geschriebenen Sprache sind solche Formate anzutreffen wie Fragewort, Fragewort mit Teilwiederholung, Teilwiederholung, copular interrogative clauses, candidate understanding und candidate reference sowie metakommunikativ explizite Fremdinitiierungen (vgl. Kap. 6.2.1). Des Weiteren kommen in der Kurznachrichtenkommunikation auch unspezifische Fremdinitiierungen vor, diese werden aber im Gegensatz zu Reparaturen in der mündlichen Kommunikation nicht zum Anzeigen eines akustischen Verstehensproblems eingesetzt, sondern erfüllen in den meisten Fällen eine andere Funktion, die ebenso im Gesprochenen belegt ist, nämlich das Markieren eines Erwartungsbruches.

Die untersuchten Reparaturdurchführungen weisen ebenfalls einige Gemeinsamkeiten mit Reparaturdurchführungen in der gesprochenen Sprache auf: Schreiber wenden bei der Bearbeitung von Problemquellen die gleichen Strategien an, wie Sprecher in der mündlichen Kommunikation, indem sie beispielsweise eine angebotene candidate solution entweder bestätigen oder ablehnen (vgl. (188) und (191)) bzw. im Falle eines Referenzproblems einen deiktischen Ausdruck mit seiner präziseren sprachlichen Form ersetzen (vgl. (183) und (185)).

Abgesehen von den oben skizzierten Überschneidungen zwischen Reparaturen in der gesprochenen Sprache und in den untersuchten Kurznachrichtendialogen haben sich im Laufe der Analyse auch einige Unterschiede herausgestellt, welche in erster Linie mit den gleichen Faktoren im Zusammenhang stehen, die bereits in 6.1.6.1 in Bezug auf selbstinitiierte Selbstreparaturen angesprochen wurden. Dabei handelt es sich also um folgende Faktoren:

(i) mediale Realisierung (vgl. Koch/Oesterreicher 1985) der drei Komponenten einer Reparatur (Stichwörter: mediale Mündlichkeit und mediale Schriftlichkeit);

(ii) technische Gegebenheiten des eigesetzten Kommunikationsmediums im Sinne von Dürscheid (2005); 
(iii) Kommunikationsbedingungen im Rahmen der Kurznachrichtenkommunikation und der Face-to-Face-Kommunikation (v.a. die wechselseitige Beziehung zwischen der zeitlichen und räumlichen Nähe bzw. Distanz und dem Synchronizitätsgrad der Kommunikation; vgl. Koch/Oesterreicher 1985 und Dürscheid 2005);

(iv) Besonderheiten der jeweiligen Kommunikations(platt)form (SMS vs. WhatsApp, Viber, iMessage).

(i) Das Analysekorpus stellt eine Sammlung aus überwiegend schriftlich repräsentierten Dialogen mit einem geringen Anteil an medial mündlichen WhatsApp-Sprachnachrichten dar (zu den dadurch bedingten Affordanzen vgl. Kap. 4 und 5; zum Konzept Affordanz vgl. auch Hutchby 2001; Zillien 2009). Davon ist lediglich eine WhatsApp-Sprachnachricht in den Untersuchungsfokus der vorliegenden Arbeit gerückt, da sie ein Element enthält, welches ein akustisches Verstehensproblem ausgelöst hat (vgl. (200)). Alleine diese Tatsache führt dazu, dass nicht alle für die gesprochene Sprache herausgearbeiteten Typen von Reparanda auf die Analyse der Kurznachrichten übertragen werden können. Vor allem betrifft das natürlich akustische Verstehensprobleme, die dadurch zustande kommen, dass ein akustisches Signal vom Rezipienten (teilweise) nicht oder nicht richtig dekodiert werden kann (vgl. Kap. 2.3.2.1). Bei Reparaturen in der textbasierten Kurznachrichtenkommunikation aus der MoCoDa ist im Gegensatz dazu nicht der auditive, sondern der visuelle Kanal involviert, sodass man nicht von einem akustischen, sondern - analog dazu gebildet - von einem ,visuellen`Verstehensproblem, bei dem der Leseprozess beeinträchtigt wird, ausgehen muss. Da aber in den Daten sowohl ein akustisches Verstehensproblem als auch einige ,visuelle‘ Verstehensprobleme vorliegen, wurden diese zwei Kategorien unter dem Oberbegriff Rezeptionsprobleme zusammengefasst (vgl. Kap. 6.2.2.1). Bei Rezeptionsproblemen, die nur die Schriftebene betreffen, ist es potenziell zwar möglich, dass ein Verstehensproblem dadurch entsteht, dass einige Elemente der Nachricht übersehen werden (vgl. die selbstinitiierte Selbstreparatur in (157)), die analysierten fremdinitiierten Selbstreparaturen zeichnen sich jedoch überwiegend dadurch aus, dass die Rezeption durch ein technisch bedingtes Problem (meist nur z.T.) gestört wurde (zur Rolle der technischen Komponente der Kommunikation vgl. Punkt (ii) des vorliegenden Kapitels).

Im Bereich der Fremdinitiierungsformate lässt sich zwar beobachten, dass viele Formate aus der gesprochenen Sprache sich in den Reparaturen aus den Kurznachrichten wiederfinden. Das betrifft u.a. Fremdinitiierungen in Form eines Fragewortes bzw. eines Fragewortes mit Teilwiederholung sowie Teilwie- 
derholungen und candidate solutions. Gleichzeitig hat die mediale Schriftlichkeit aber die Entwicklung neuer unikaler Initiierungsressourcen ermöglicht und begünstigt. Dabei handelt es sich v.a. um den Einsatz solcher Mittel, die in der medial mündlichen Kommunikation nicht möglich sind, nämlich um einzelne Interpunktionszeichen bzw. mathematische Zeichen wie Fragezeichen (vgl. Kap. 6.2.1.1), Asteriske (vgl. (200)) sowie Gleichheitszeichen (vgl. (194)).

Der zweite durch die Schriftlichkeit ermöglichte Aspekt in Bezug auf die Fremdinitiierungen von Reparaturen hängt eng mit den Produktionsbedingungen der Kurznachrichtenkommunikation zusammen, die i.d.R. eine prompte Reaktion erfordern. Um diesen Kommunikationsbedingungen gerecht zu werden, greifen Schreiber u.a. auf diverse Abkürzungen sowie Kurzformen als Ökonomisierungsverfahren (vgl. Androutsopoulos 2007: 82-83) zurück. Abgekürzte Varianten der Fremdinitiierungen finden sich aber nur im russischen Subkorpus (dabei handelt es sich um die bereits konventionalisierte Abkürzung r.e. (,d.h.') in (190) sowie um die nicht-standardsprachliche Form всм (für в смысле ,im Sinne von') in (170) und (171)). Dafür weisen im deutschen Subkorpus Reparaturdurchführungen die Abkürzung d.h. (vgl. \#2404 in der MoCoDa) auf, wobei auch die Fremdinitiierung mit $d$.h. für die deutschen Daten denkbar wäre. Auch die Verwendung der Emojis in (226) und (227), die jeweils eine reparative Funktion ausüben, lässt sich als Ökonomisierungsstrategie betrachten.

(ii) Eine wichtige Rolle für die Analyse der Reparaturen in der Kurznachrichtenkommunikation spielt auch das jeweilige Kommunikationsmedium im Sinne von Dürscheid (2005). Dazu zählen v.a. Handys und Smartphones, wobei man Messenger-Apps auch auf einem Laptop, PC oder Tablet installieren kann, sodass man z.B. WhatsApp-Nachrichten auch darüber verschicken kann. Möchte man den Kontrast zwischen den Reparaturen im Gesprochenen und Geschriebenen aufzeigen, fällt auf, dass es sinnvoll wäre, für die gesprochene Sprache zwischen Reparaturen in Face-to-Face-Interaktionen und in ,mittelbaren', ein Kommunikationsmedium benötigten Interaktionen zu unterscheiden, da im zweiten Fall das entsprechende Kommunikationsmedium das Gespräch mitprägt. So kann beispielsweise ein Videoanruf via Skype oder ein Telefongespräch durch eine Signalstörung kurz unterbrochen werden und logischerweise zum Entstehen bestimmter Verstehens- und Verständigungsproblemen (v.a. akustischer Verstehensproblemen) führen.

In Bezug auf die fremdinitiierten Selbstreparaturen in der Kurznachrichtenkommunikation kann der Einfluss der technischen Eigenschaften eines Kommunikationsmediums aus zwei Perspektiven betrachtet werden, nämlich aus der Perspektive des Absenders und des Empfängers der Nachricht. Im ersten Fall wird die Nachricht vom Absender eingegeben, von dessen Gerät dement- 
sprechend kodiert und an den Rezipienten übermittelt. Hierzu gehören solche Fälle, bei denen durch eine aktivierte Autokorrektur eine oder mehrere Konstituenten in der Nachricht verändert werden, sodass dadurch eine Problemquelle für den Rezipienten entsteht (vgl. (178), (204) und (209)). Des Weiteren kommt in den untersuchten Daten ein Fall (vgl. (210)) vor, bei dem eine Nachricht per Sprachbefehl abgeschickt wird. Dabei wird ein Teil der mündlichen Äußerung von einer Spracherkennungssoftware jedoch nicht korrekt erkannt und somit falsch in die Textform überführt, sodass die aufgenommene Phrase „dein Vater“ als „googoo“ dargestellt wird.

Selbst dann, wenn eine Nachricht in korrekter und erwünschter Form verfasst und verschickt wurde, kann sie oder einige ihrer Elemente aus der Sicht des Rezipienten ein Verstehens- oder Verständigungsproblem enthalten. Das kommt v.a. dadurch zustande, dass das Gerät des Rezipienten den Inhalt der Nachricht empfangen, dekodieren und korrekt darstellen muss. Das erste Problem kann bereits beim Empfang einer Nachricht entstehen, und zwar dann, wenn eine SMS-Nachricht nicht komplett von dem Endgerät des Empfängers dargestellt werden kann, sodass z.B. das Ende der Nachricht fehlt (vgl. (201)). Ein weiteres Problem, das im Untersuchungskorpus ebenso in einer SMS-Nachricht aufgetreten ist, betrifft die Entschlüsselung der erhaltenen Nachricht auf dem Endgerät. Dabei stellt das in Kap. 6.2.2.1 analysierte Beispiel (202) einen Extremfall dar, bei dem die komplette Nachricht nicht richtig abgebildet werden konnte, da das Endgerät offensichtlich Probleme mit der Darstellung der kyrillischen Schrift hatte, sodass die Kommunikation in der lateinischen Umschrift fortgeführt werden musste. Für die WhatsApp-Kommunikation könnte es potenziell ebenso zu Problemen bei der Darstellung der Nachrichteninhalte kommen, wenn der Absender und der Empfänger unterschiedliche Versionen der WhatsApp-Software haben. Das würde aber vermutlich nicht den Text der Nachricht selbst, sondern eher die Darstellung von Emojis, Stickern bzw. GIFs betreffen.

(iii) Ein weiterer Faktor, von dem die Reparaturen in der Kurznachrichtenkommunikation geprägt sind, hängt mit der Kommunikationssituation zusammen. Die Interaktion verläuft unter Bedingungen zeitlicher und räumlicher Distanz. Die Tatsache, dass die Kommunikationspartner nicht über den geteilten physischen Raum verfügen sowie sich manchmal auch in unterschiedlichen Zeitzonen befinden, begünstigt das Auftreten von Referenzproblemen (vgl. (163)). Zudem führen die räumliche Distanz sowie das Ausbleiben des auditiven Kommunikationskanals dazu, dass in Einzelfällen eine Nachricht an einen falschen Adressaten verschickt wird (vgl. \#3189 und \#3482 in der MoCoDa), was in einem Face-to-Face-Gespräch durch die Anwesenheit des Interaktionspartners 
ausgeschlossen werden kann. In einem Telefongespräch dagegen kann es potenziell dazu kommen (ein Beispiel - allerdings für eine selbstinitiierte Selbstreparatur bei der Verwechslung des Adressaten - führt Schegloff (1992: 1322) an). Eine Begrüßungssequenz bzw. das Erkennen der Stimme des Gegenübers mindern aber die Wahrscheinlichkeit eines solchen Problems. Des Weiteren führt die räumliche Distanz in Kombination mit fehlenden Anhaltspunkten für die Identifikation des Interaktionspartners, die weder durch die Stimme des Gegenübers (wie bei einem Telefonat) noch durch die gespeicherte Telefonnummer möglich ist, dazu, dass, bevor die Kommunikation weitergehen kann, der Kommunikationspartner im Rahmen einer Fremdinitiierungen identifiziert werden muss (vgl. \#2947 und \#3782 in der MoCoDa).

(iv) Als Letztes soll nun kurz auf die Frage nach der Rolle der jeweiligen Kommunikations(platt)form - SMS-Kommunikation und Kommunikation via Messenger - eingegangen werden. In der vorliegenden Arbeit wurden diese zwei Kommunikationsformen v.a. aus praktischen Gründen zusammengelegt und mit dem übergeordneten Begriff Kurznachrichtenkommunikation bezeichnet. Diese Entscheidung lag v.a. an der Datenzusammensetzung, da die MoCoDa neben SMS-, WhatsApp-, Viber- und iMessage-Dialogen einige Dialogsequenzen enthält, deren Metadaten leider keine Informationen über deren Zuordnung zu einer der Kommunikations(platt)formen beinhalten (vgl. Kap. 5).

Für weitere Forschungsarbeiten könnte man aber fremdinitiierte Selbstreparaturen in der SMS- und Messenger-Kommunikation getrennt voneinander untersuchen und ausführlicher auf deren Unterschiede und Gemeinsamkeiten eingehen. In Bezug auf die WhatsApp-Kommunikation, die im Vergleich zur SMS-Kommunikation über umfangreichere technische Funktionen verfügt, könnte man beispielsweise folgenden Forschungsfragen nachgehen: Wird bei Reparaturinitiierungen bzw. -durchführungen von der Möglichkeit Gebrauch gemacht, eine eigene oder fremde Nachricht als ,Zitat' in eine neue Nachricht einzubauen? Wie wird mit gelöschten Nachrichten umgegangen? Werden die Inhalte solcher Nachrichten im weiteren Verlauf von Rezipienten als Problemquellen thematisiert? Oder hat sich bereits eine kommunikative Praktik entwickelt, solche Nachrichten als versehentlich verschickte anzusehen und somit zu ignorieren? Inwiefern werden variable multimediale Ressourcen wie Emojis, Sticker, GIFs, Fotos, Standortinformationen, Screenshots, Videos etc. in der Messenger-Kommunikation zu Reparaturzwecken eingesetzt? usw.

\subsubsection{Fremdinitiierte Selbstreparaturen im Russischen und Deutschen}

In sprachkontrastiver Hinsicht lässt sich bei den untersuchten fremdinitiierten Selbstreparaturen eine ähnliche Tendenz wie bei in 6.1.6.2 diskutierten selbst- 
initiierten Selbstreparaturen beobachten: Dabei liegen ebenfalls sowohl Unterschiede als auch Gemeinsamkeiten in Bezug auf die bearbeiteten Problemquellen, die Initiierungsformate sowie die eingesetzten Reparaturoperationen vor. Als Problemquellen treten beispielswiese in beiden Korpora Rezeptions-, Referenz-, Bedeutungsverstehens-, Erwartungsprobleme sowie Probleme, die von der problematischen sequenziellen Implikation ausgelöst werden, auf (vgl. Kap. 6.2.2).

Im Bereich der Initiierungsformate lässt sich ebenso eine Reihe von Parallelen zwischen Fremdinitiierungen in den beiden untersuchten Sprachen erkennen. Beispielsweise benutzen Schreiber Formate wie Fragewörter (vgl. Kap. 6.2.1.3) und Teilwiederholungen (vgl. Kap. 6.2.1.4). Zudem wurde der Gebrauch von Fragezeichen als einer ausschließlich in der medialen Schriftlichkeit möglichen Fremdinitiierung, die v.a. vorliegende Bedeutungsverstehens- und Erwartungsprobleme signalisiert, sowohl im russischen als auch im deutschen Subkorpus belegt. Im Gegensatz $\mathrm{zu}$ Fragezeichen geht die Verwendung unspezifischer Initiierungsformate wie unspezifische Fragewörter sowie Interjektionen auseinander. Während in den deutschen Daten beide Ressourcen vertreten sind, weist das russische Subkorpus lediglich ein einziges Vorkommen des Fragewortes что (,was') auf (vgl. (162)). Dieser Befund bietet Anknüpfungspunkte für weitere Untersuchungen der Reparaturen in der informellen interaktionalen Schriftlichkeit, um herauszufinden, ob und inwiefern unspezifische Reparaturinitiierungen in der russischen CMC vertreten sind. Andererseits scheint auch eine vertiefte Analyse der Funktionen solcher Fremdinitiierungen in der russischen gesprochenen Sprache vonnöten zu sein, um v.a. die damit angezeigten Problemquellen zu ermitteln und festzustellen, ob und - falls ja welche weiteren Problemquellen außer akustischen Verstehensproblemen beispielsweise mit den Partikeln $a$ (,hä‘) oder $M($,hm') angezeigt werden können.

Des Weiteren können auch candidate solutions in der jeweiligen Sprache anhand eines größeren Datenkorpus unter die Lupe genommen werden. Hierbei könnte man zum einen entweder einzelne sprachspezifische sprachliche Mittel herausarbeiten oder sich auf parallele Konstruktionen konzentrieren, die in beiden Sprachen existieren und auf deren (potenziellen) reparativen Gebrauch hin untersucht werden müssen. Dafür würden sich u.a. candidate solutions mit Was für X? Im Sinne von X? und Meinst du X? für das Deutsche und deren russische Entsprechungen Что за Х? (vgl. Podlesskaya 2007), В смысле (X)? (vgl. Goletiani 2003; vgl. auch (169) und (228)) sowie Имеешь в виду Х? (vgl. Goletiani 2003: 365-369).

Während bei den oben erwähnten Initiierungsformaten gewisse Ähnlichkeiten in den beiden Teilkorpora beobachtet wurden bzw. diese zumindest poten- 
ziell möglich sind, kommt in den deutschen Kurznachrichten ein Initiierungsformat vor, dessen Pendant im Russischen aus sprachsystematischen Gründen einen anderen syntaktischen Aufbau aufweist. Bei diesem Initiierungsformat handelt es sich um die sogenannten copular interrogative clauses, die aus einem Fragewort, einem Kopulaverb und einem Verweis auf das Reparandum bestehen, wobei das Reparandum entweder mit einem Demonstrativpronomen (z.B. der) oder durch die Wiederholung wiederaufgenommen werden kann. Da sich die Satzbauregeln im Russischen von denen des Deutschen unterscheiden und einer der Unterschiede darin besteht, dass viele russische Sätze ohne Verb realisiert werden (können), benötigt man bei dem mit deutschen copular interrogative clauses vergleichbaren Format im Russischen kein Kopulaverb. Darüber hinaus zeichnet sich das russische Initiierungsformat dadurch aus, dass das Demonstrativpronomen это (,das') als obligatorische Konstituente der Fremdinitiierung fungiert und sowohl eine voran- als auch nachgestellte Position in Bezug auf das realisierte Fragewort annehmen kann (это (,das') + Fragewort bzw. Fragewort + это (,das')). Das Reparandum selbst kann zwar in der Fremdinitiierung erscheinen, dieses wird aber im Gegensatz zum Deutschen als eine Linksversetzung realisiert (vgl. (185)).

Eine weitere Forschungsfrage für zukünftige Untersuchungen stellt der Gebrauch nonverbaler graphischer Fremdinitiierungen unter sprachvergleichender Perspektive dar. Die vorliegende Untersuchung belegt zwar, dass Fragezeichen in beiden Sprachen als Fremdinitiierungen fungieren können, das Potenzial weiterer graphischer Zeichen, wie z.B. Gleichheitszeichen oder Asteriske, die je mit einem Token im deutschen Subkorpus belegt sind, muss aber anhand größerer Korpora erforscht werden (vgl. dazu auch die Studie von Mostovaia (2018) zu graphischen Reparaturinitiierungen in der WhatsApp- und Chat-Kommunikation auf der Basis von Daten aus der MoCoDa, der MoCoDa 2 und dem Dortmunder Chat-Korpus).

In Bezug auf Reparaturdurchführungen lassen sich bei impliziten und expliziten Reparaturdurchführungen im Deutschen und Russischen miteinander überschneidende Strategien beobachten. Es wurde bereits in 6.2.3.1 ausgeführt, dass Referenzprobleme in den beiden Teilkorpora oft bearbeitet werden, indem die problematische Referenz präzisiert wird, während vorgelegte candidate solutions entweder bestätigt oder abgelehnt und korrigiert werden. Ergänzend könnte man bei Reparaturdurchführungen auf konkrete sprachliche Mittel näher eingehen, mit denen sie in der jeweiligen Sprache vollzogen werden können.

Außer den drei sequenziellen Komponenten einer Reparatur wurde in 6.2.5 kurz auf die formalen und funktionalen Eigenschaften von Emoticons und Emo- 
jis eingegangen, die Reparaturinitiierungen und -durchführungen in den beiden Teilkorpora begleiten. In dieser Hinsicht haben sich ebenso einige Gemeinsamkeiten sowohl bei den verwendeten Emojis und Emoticons als auch bei den von ihnen ausgeübten Funktionen herausgestellt. In den Analysedaten fällt beispielsweise auf, dass im Deutschen sowohl bei Fremdinitiierungen als auch bei Reparaturdurchführungen das Emoticon $: D$ den ersten und :) den zweiten Platz einnehmen, während in den russischen Dialogen :) und seine Varianten am häufigsten vertreten sind. Leider lässt die Größe der Untersuchungskorpora nicht darauf schließen, ob diese Distribution einen systematischen Charakter hat und auf den Gebrauch von Emojis und Emoticons in der jeweiligen Sprache zurückzuführen ist. 